\title{
Dietary Supplement Laboratory Quality Assurance Program: Exercise N Final Report
}

\author{
Charles A. Barber \\ Melissa M. Phillips \\ Catherine A. Rimmer \\ Laura J. Wood \\ Steven J. Christopher
}




\title{
Dietary Supplement Laboratory Quality Assurance Program: Exercise N Final Report
}

\author{
Charles A. Barber \\ Melissa M. Phillips \\ Catherine A. Rimmer \\ Laura J. Wood \\ Steven J. Christopher \\ Chemical Sciences Division \\ Material Measurement Laboratory
}

This publication is available free of charge from: https://doi.org/10.6028/NIST.IR.8230r1

October 2018

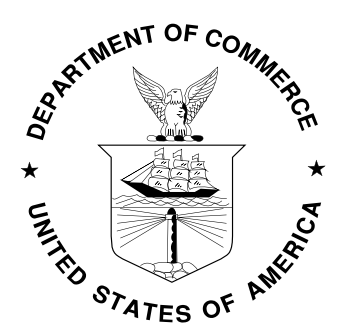

U.S. Department of Commerce Wilbur L. Ross, Jr., Secretary

National Institute of Standards and Technology Walter Copan, NIST Director and Under Secretary of Commerce for Standards and Technology 


\section{TABLE OF CONTENTS}

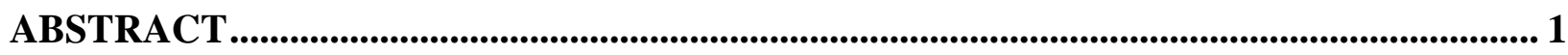

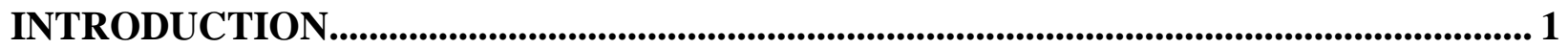

OVERVIEW OF DATA TREATMENT AND REPRESENTATION ................................... 2

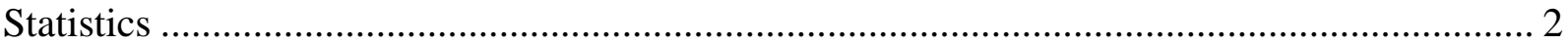

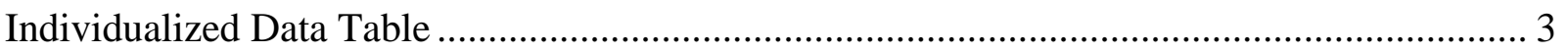

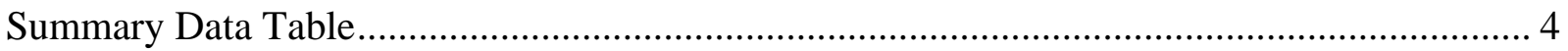

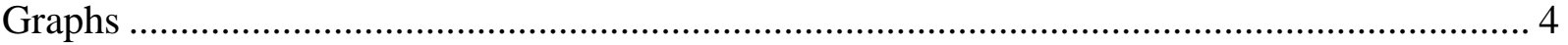

Data Summary View (Method Comparison Data Summary View) ......................................... 4

Sample/Sample Comparison View ............................................................................ 5

NUTRITIONAL ELEMENTS (Cr) IN MULTIVITAMIN AND CHROMIUM DIETARY

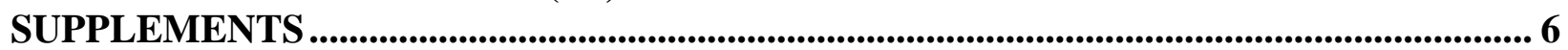

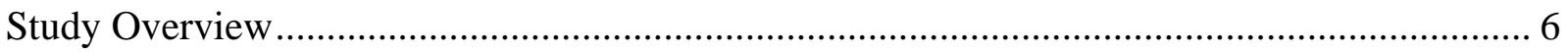

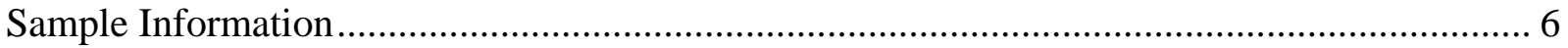

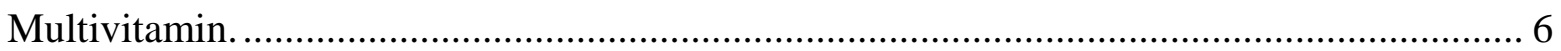

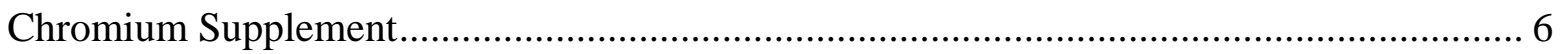

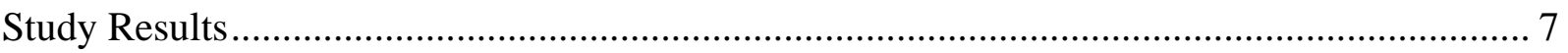

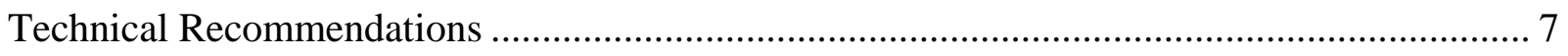

Table 1. Individualized data summary table (NIST) for chromium in multivitamin and chromium supplement. ................................................................................................... 8

Table 2. Data summary table for chromium in multivitamin and a chromium supplement. . 9

Figure 1. Chromium in SRM 3280 Multivitamin/Multielement Tablets (data summary view - analytical method).

Figure 2. Chromium in candidate SRM 3279 Chromium-Containing Solid Oral Dosage Form (data summary view - analytical method)

Figure 3. Laboratory means for chromium in SRM 3280 Multivitamin/Multielement Tablets and candidate SRM 3279 Chromium-Containing Solid Oral Dosage Form (sample/sample comparison view).

TOXIC ELEMENTS (As, Cd, AND Pb) IN CALCIUM AND GINKGO DIETARY

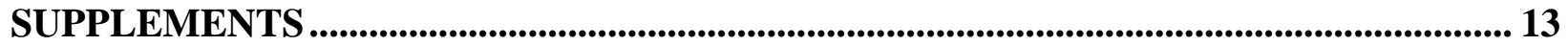

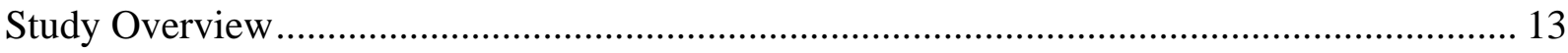

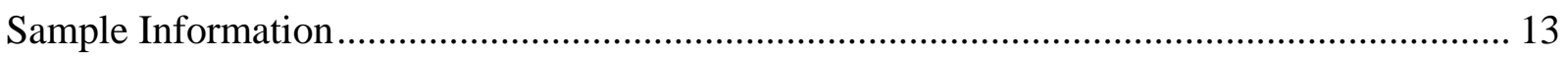

Calcium Supplement ………………………………………………………………... 13

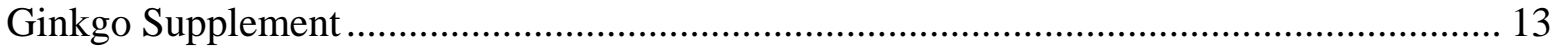




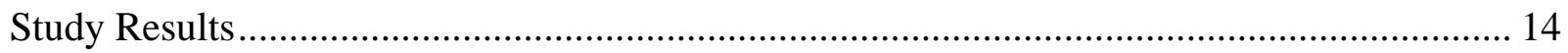

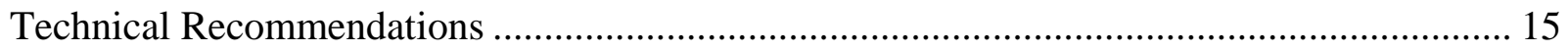

Table 3. Individualized data summary table (NIST) for arsenic, cadmium, and lead in calcium

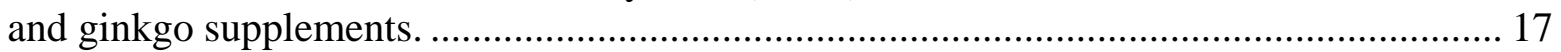

Table 4. Data summary table for total arsenic in calcium and ginkgo supplements. .......... 18

Table 5. Data summary table for cadmium in calcium and ginkgo supplements............... 19

Table 6. Data summary table for lead in calcium and ginkgo supplements....................... 20

Figure 4. Arsenic in SRM 3532 Calcium-Containing Solid Oral Dosage Form (data summary

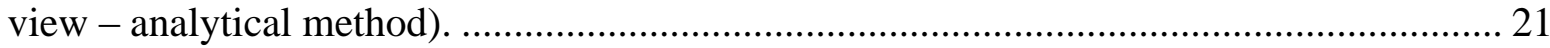

Figure 5. Arsenic in SRM 3248 Ginkgo-Containing Tablets (data summary view - analytical

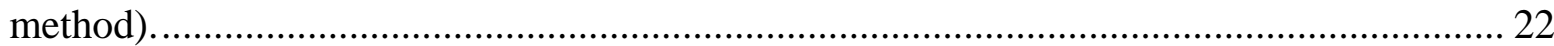

Figure 6. Cadmium in SRM 3532 Calcium-Containing Solid Oral Dosage Form (data

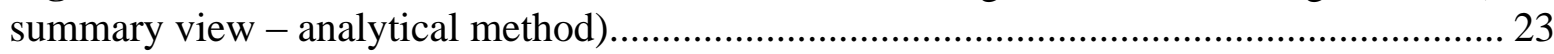

Figure 7. Cadmium in SRM 3248 Ginkgo-Containing Tablets (data summary view -

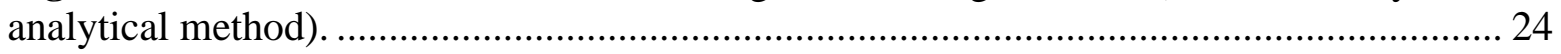

Figure 8. Lead in SRM 3532 Calcium-Containing Solid Oral Dosage Form (data summary

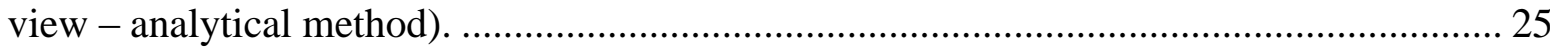

Figure 9. Lead in SRM 3248 Ginkgo-Containing Tablets (data summary view - analytical

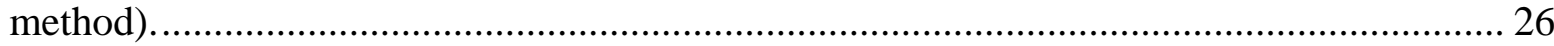

Figure 10. Laboratory means for total arsenic in SRM 3532 Calcium-Containing Solid Oral Dosage Form and SRM 3248 Ginkgo-Containing Tablets (sample/sample comparison view).

Figure 11. Laboratory means for cadmium in SRM 3532 Calcium-Containing Solid Oral Dosage Form and SRM 3248 Ginkgo-Containing Tablets (sample/sample comparison view). 28

Figure 12. Laboratory means for lead in SRM 3532 Calcium-Containing Solid Oral Dosage Form and SRM 3248 Ginkgo-Containing Tablets (sample/sample comparison view). ....... 29

\section{WATER-SOLUBLE VITAMINS (FOLIC ACID) IN FOODS AND DIETARY SUPPLEMENTS ......................................................................................................................... 30}

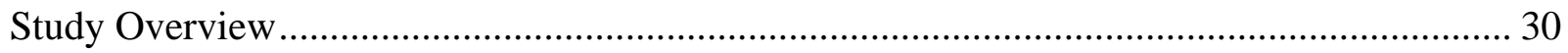

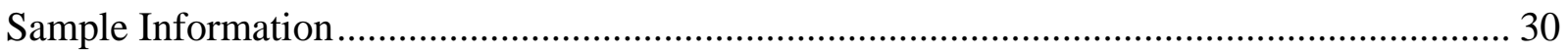

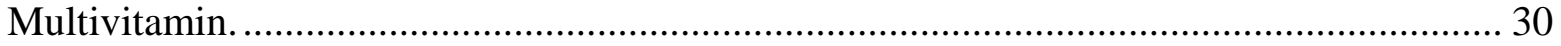

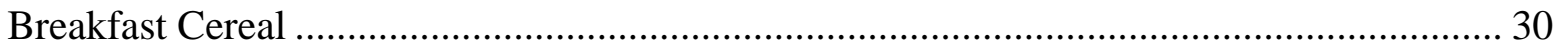

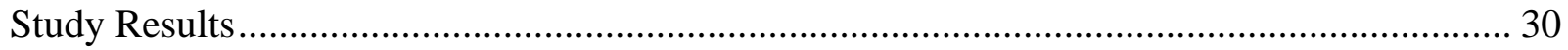

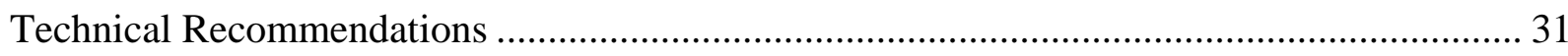

Table 7. Individualized data summary table (NIST) for folic acid in multivitamin and

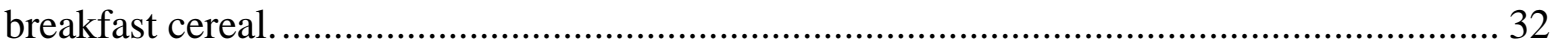


Table 8. Data summary table for folic acid in multivitamin and in breakfast cereal.......... 33

Figure 13. Folic acid in SRM 3280 Multivitamin/Multielement Tablets (data summary view - analytical method).

Figure 14. Folic acid in SRM 3233 Fortified Breakfast Cereal (data summary view analytical method).

Figure 15. Laboratory means for folic acid in SRM 3280 Multivitamin/Multielement Tablets and SRM 3233 Fortified Breakfast Cereal (sample/sample comparison view). 36

FAT-SOLUBLE VITAMINS (VITAMIN $D_{2}$ AND $D_{3}$ ) IN CALCIUM, PROTEIN, AND MULTIVITAMIN DIETARY SUPPLEMENTS ........................................................ 37

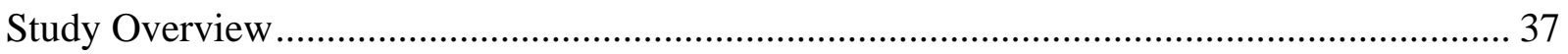

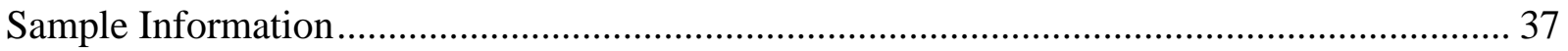

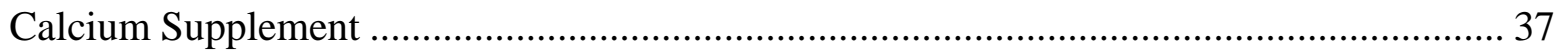

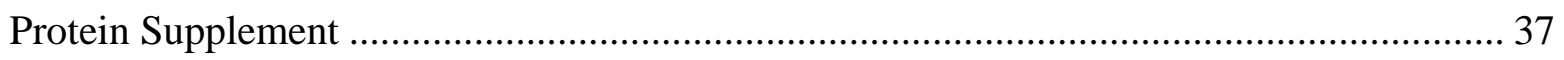

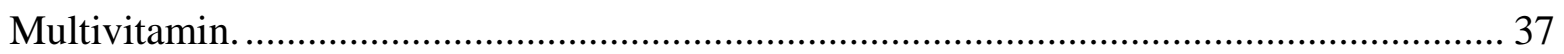

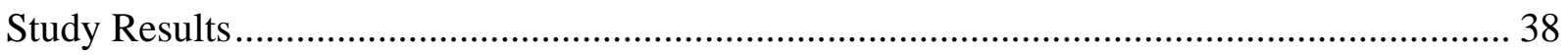

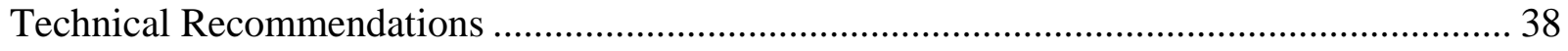

Table 9. Individualized data summary table (NIST) for ergocalciferol (vitamin $\mathrm{D}_{2}$ ) and cholecalciferol (vitamin $\mathrm{D}_{3}$ ) in dietary supplements. ........................................... 40

Table 10. Data summary table for ergocalciferol (vitamin D2) in dietary supplements.... 41

Table 11. Data summary table for cholecalciferol (vitamin D3) in dietary supplements.... 42

Figure 16. Total ergocalciferol (vitamin $\mathrm{D}_{2}$ ) in SRM 3532 Calcium-Containing Solid Oral Dosage Form (data summary view - analytical method)........................................ 43

Figure 17. Total ergocalciferol (vitamin $\mathrm{D}_{2}$ ) in SRM 3252 Protein Drink Mix (data summary

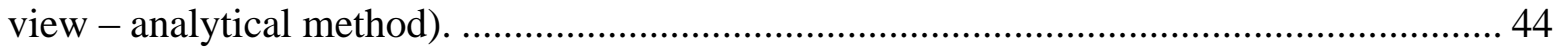

Figure 18. Total ergocalciferol (vitamin $\mathrm{D}_{2}$ ) in SRM 3280 Multivitamin/Multielement Tablets (data summary view - analytical method). 45

Figure 19. Total cholecalciferol (vitamin $D_{3}$ ) in SRM 3532 Calcium-Containing Solid Oral Dosage Form (data summary view - analytical method)........................................ 46

Figure 20. Total cholecalciferol (vitamin $D_{3}$ ) in SRM 3252 Protein Drink Mix (data summary view - analytical method).

Figure 21. Total cholecalciferol (vitamin $\mathrm{D}_{3}$ ) in SRM 3280 Multivitamin/Multielement Tablets (data summary view - analytical method).

Figure 22. Laboratory means for ergocalciferol (vitamin $D_{2}$ ) in SRM 3280 Multivitamin/Multielement Tablets and SRM 3252 Protein Drink Mix (sample/sample comparison view).

Figure 23. Laboratory means for cholecalciferol (vitamin $\mathrm{D}_{3}$ ) in SRM 3280 Multivitamin/Multielement Tablets and SRM 3252 Protein Drink Mix (sample/sample comparison view). 
Figure 24. Laboratory means for cholecalciferol (vitamin $D_{3}$ ) in SRM 3280 Multivitamin/Multielement Tablets and SRM 3532 Calcium-Containing Solid Oral Dosage Form (sample/sample comparison view)

Figure 25. Laboratory means for cholecalciferol (vitamin D3) in SRM 3252 Protein Drink Mix and SRM 3532 Calcium-Containing Solid Oral Dosage Form (sample/sample comparison view).

FATTY ACIDS IN SAW PALMETTO BERRIES AND BORAGE OIL DIETARY SUPPLEMENTS

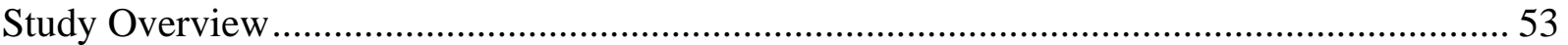

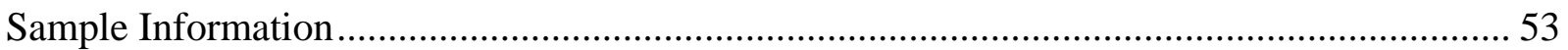

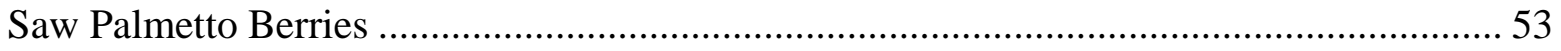

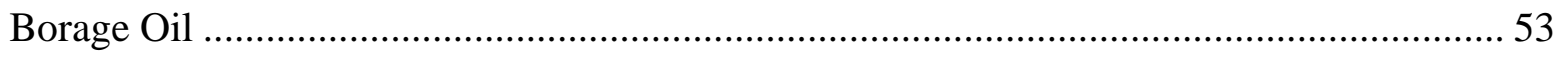

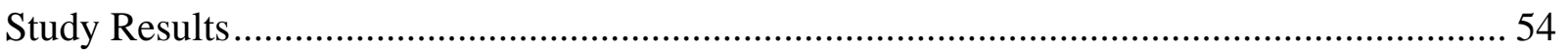

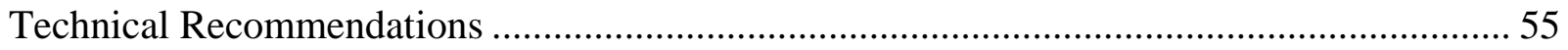

Table 12. Individualized data summary table (NIST) for fatty acids in dietary supplements. 56

Table 13. Data summary table for $\alpha$-linolenic acid (as FAME) in dietary supplements..... 57

Table 14. Data summary table for $\gamma$-linolenic (as FAME) acid in dietary supplements. .... 58

Table 15. Data summary table for linoleic acid (as FAME) in dietary supplements. ......... 59

Table 16. Data summary table for total omega-3 fatty acids (as FAMEs) in dietary supplements. Data highlighted in red have been flagged as potential outliers (e.g., difference from reference value, Grubb and/or Cochran) by the NIST software package. 60

Table 17. Data summary table for total omega-6 fatty acids (as FAMEs) in dietary supplements. Data highlighted in red have been flagged as potential outliers (e.g., difference from reference value, Grubb and/or Cochran) by the NIST software package. 61

Figure 26. $\alpha$-Linolenic acid (as FAME) in SRM 3250 Saw Palmetto (Serenoa repens) Fruit (data summary view - analytical method).

Figure 27. $\alpha$-Linolenic acid (as FAME) in SRM 3274-1 Borage (Borago officinalis) Oil (data summary view - analytical method). 63

Figure 28. $\gamma$-Linolenic acid (as FAME) in SRM 3250 Saw Palmetto (Serenoa repens) Fruit (data summary view - analytical method). 64

Figure 29. $\gamma$-Linolenic acid (as FAME) in SRM 3274-1 Borage (Borago officinalis) Oil (data summary view - analytical method). 65

Figure 30. Linoleic acid (as FAME) in SRM 3250 Saw Palmetto (Serenoa repens) Fruit (data summary view - analytical method). 66

Figure 31. Linoleic acid (as FAME) in SRM 3274-1 Borage (Borago officinalis) Oil (data summary view - analytical method).... 67 
Figure 32. Total omega-3 fatty acids (as FAMEs) in SRM 3250 Saw Palmetto (Serenoa

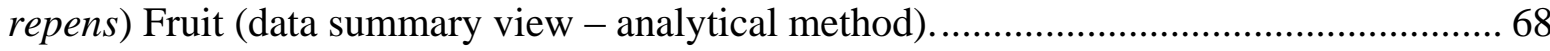

Figure 33. Total omega-3 fatty acids (as FAMEs) in SRM 3274-1 Borage (Borago officinalis) Oil (data summary view - analytical method).

Figure 34. Total omega-6 fatty acids (as FAMEs) in SRM 3250 Saw Palmetto (Serenoa repens) Fruit (data summary view - analytical method). 70

Figure 35. Total omega-6 fatty acid (as FAMEs) in SRM 3274-1 Borage (Borago officinalis) Oil (data summary view - analytical method). 71

Figure 36. Laboratory means for $\alpha$-linolenic acid (as FAME) in SRM 3250 Saw Palmetto (Serenoa repens) Fruit, and SRM 3274-1 Borage (Borago officinalis) Oil (sample/sample comparison view). 72

Figure 37. Laboratory means for $\gamma$-linolenic acid (as FAME) in SRM 3250 Saw Palmetto (Serenoa repens) Fruit, and SRM 3274-1 Borage (Borago officinalis) Oil (sample/sample comparison view). 73

Figure 38. Laboratory means for linolenic acid (as FAME) in SRM 3250 Saw Palmetto (Serenoa repens) Fruit, and SRM 3274-1 Borage (Borago officinalis) Oil (sample/sample comparison view). 74

Figure 39. Laboratory means for total omega-3 fatty acids (as FAMEs) in SRM 3250 Saw Palmetto (Serenoa repens) Fruit, and SRM 3274-1 Borage (Borago officinalis) Oil

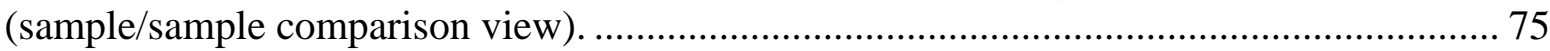

Figure 40. Laboratory means for total omega-6 fatty acids (as FAMEs) in SRM 3250 Saw Palmetto (Serenoa repens) Fruit, and SRM 3274-1 Borage (Borago officinalis) Oil (sample/sample comparison view). 76

\section{GINSENOSIDES IN GROUND ASIAN GINSENG EXTRACT AND RHIZOME BOTANICAL SUPPLEMENTS........................................................................................................... 77}

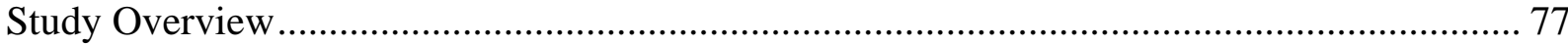

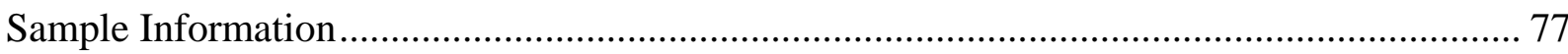

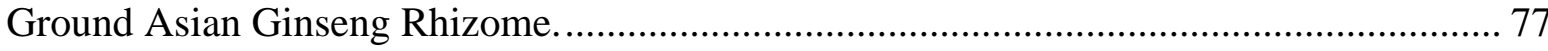

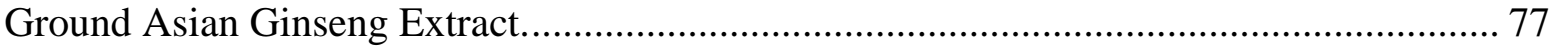

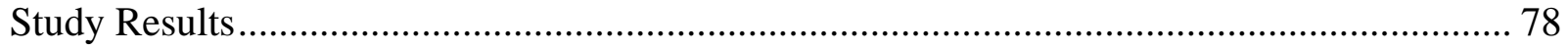

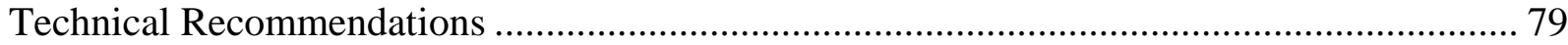

Table 18. Individualized data summary table (NIST) for ginsenosides in botanical

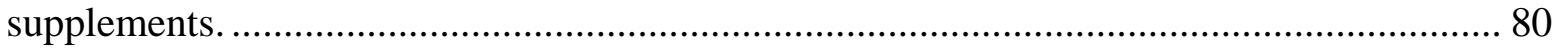

Table 19. Data summary table for ginsenoside Rb1 in botanical supplements. Data highlighted in red have been flagged as potential outliers (e.g., difference from reference value, Grubb and/or Cochran) by the NIST software package. 81

Table 20. Data summary table for ginsenoside $\mathrm{Rb} 2$ in botanical supplements. Data highlighted in red have been flagged as potential outliers (e.g., difference from reference value, Grubb and/or Cochran) by the NIST software package. 82 
Table 21. Data summary table for ginsenoside Rc in botanical supplements. Data highlighted in red have been flagged as potential outliers (e.g., difference from reference value, Grubb and/or Cochran) by the NIST software package. 83

Table 23. Data summary table for ginsenoside Re in botanical supplements. Data highlighted in red have been flagged as potential outliers (e.g., difference from reference value, Grubb and/or Cochran) by the NIST software package. 85

Table 24. Data summary table for ginsenoside Rf in botanical supplements. Data highlighted in red have been flagged as potential outliers (e.g., difference from reference value, Grubb and/or Cochran) by the NIST software package. 86

Table 25. Data summary table for ginsenoside $\operatorname{Rg} 1$ in botanical supplements. Data highlighted in red have been flagged as potential outliers (e.g., difference from reference value, Grubb and/or Cochran) by the NIST software package. 87

Table 26. Data summary table for ginsenoside $\operatorname{Rg} 2$ in botanical supplements. Data highlighted in red have been flagged as potential outliers (e.g., difference from reference value, Grubb and/or Cochran) by the NIST software package. 88

Figure 41. Ginsenoside Rb1 in candidate SRM 3384 Ground Asian Ginseng (Panax ginseng C.A. Meyer) Rhizome (data summary view - analytical method). 89

Figure 42. Ginsenoside Rb1 in candidate SRM 3385 Ground Asian Ginseng (Panax ginseng C.A. Meyer) Extract (data summary view - analytical method). 90

Figure 43. Ginsenoside Rb2 in candidate SRM 3384 Ground Asian Ginseng (Panax ginseng C.A. Meyer) Rhizome (data summary view - analytical method). 91

Figure 44. Ginsenoside Rb2 in candidate SRM 3385 Ground Asian Ginseng (Panax ginseng C.A. Meyer) Extract (data summary view - analytical method). 92

Figure 45. Ginsenoside Rc in candidate SRM 3384 Ground Asian Ginseng (Panax ginseng C.A. Meyer) Rhizome (data summary view - analytical method). 93

Figure 46. Ginsenoside Rc in candidate SRM 3385 Ground Asian Ginseng (Panax ginseng C.A. Meyer) Extract (data summary view - analytical method). 94

Figure 47. Ginsenoside Rd in candidate SRM 3384 Ground Asian Ginseng (Panax ginseng C.A. Meyer) Rhizome (data summary view - analytical method). 95

Figure 48. Ginsenoside Rd in candidate SRM 3385 Ground Asian Ginseng (Panax ginseng C.A. Meyer) Extract (data summary view - analytical method). 96

Figure 49. Ginsenoside Re in candidate SRM 3384 Ground Asian Ginseng (Panax ginseng C.A. Meyer) Rhizome (data summary view - analytical method). 97

Figure 50. Ginsenoside Re in candidate SRM 3385 Ground Asian Ginseng (Panax ginseng C.A. Meyer) Extract (data summary view - analytical method). 98

Figure 51. Ginsenoside Rf in candidate SRM 3384 Ground Asian Ginseng (Panax ginseng C.A. Meyer) Rhizome (data summary view - analytical method). 99

Figure 52. Ginsenoside Rf in candidate SRM 3385 Ground Asian Ginseng (Panax ginseng C.A. Meyer) Extract (data summary view - analytical method). 100 
Figure 53. Ginsenoside Rg1 in candidate SRM 3384 Ground Asian Ginseng (Panax ginseng C.A. Meyer) Rhizome (data summary view - analytical method)................................. 101

Figure 54. Ginsenoside Rg1 in SRM 3385 Ground Asian Ginseng (Panax ginseng C.A. Meyer) Extract (data summary view - analytical method). 102

Figure 55. Ginsenoside $\operatorname{Rg} 2$ in candidate SRM 3384 Ground Asian Ginseng (Panax ginseng C.A. Meyer) Rhizome (data summary view - analytical method). 103

Figure 56. Ginsenoside $\operatorname{Rg} 2$ in candidate SRM 3385 Ground Asian Ginseng (Panax ginseng C.A. Meyer) Extract (data summary view - analytical method). 104

Figure 57. Laboratory means for ginsenoside Rb1 in Asian ginseng rhizome and Asian ginseng extract (sample/sample comparison view). 105

Figure 58. Laboratory means for ginsenoside $\mathrm{Rb} 2$ in Asian ginseng rhizome and Asian ginseng extract (sample/sample comparison view). 106

Figure 59. Laboratory means for ginsenoside Rc in Asian ginseng rhizome and Asian ginseng extract (sample/sample comparison view). 107

Figure 60. Laboratory means for ginsenoside $\mathrm{Rd}$ in Asian ginseng rhizome and Asian ginseng extract (sample/sample comparison view). 108

Figure 61. Laboratory means for ginsenoside Re in Asian ginseng rhizome and Asian ginseng extract (sample/sample comparison view). 109

Figure 62. Laboratory means for ginsenoside $\mathrm{Rf}$ in Asian ginseng rhizome and Asian ginseng extract (sample/sample comparison view). 110

Figure 63. Laboratory means for ginsenoside $\operatorname{Rg} 1$ in Asian ginseng rhizome and Asian ginseng extract (sample/sample comparison view). 111

Figure 64. Laboratory means for ginsenoside $\mathrm{Rg} 2$ in Asian ginseng rhizome and Asian ginseng extract (sample/sample comparison view). 112 


\begin{abstract}
The NIST Dietary Supplement Laboratory Quality Assurance Program (DSQAP) was established in collaboration with the National Institutes of Health (NIH) Office of Dietary Supplements (ODS) in 2007 to enable members of the dietary supplement community to improve the accuracy of measurements for demonstration of compliance with various regulations, including the dietary supplement current good manufacturing practices (cGMPs). Exercise $\mathrm{N}$ of this program offered the opportunity for laboratories to assess their in-house measurements of nutritional elements (chromium), contaminants (arsenic, cadmium, and lead), water-soluble vitamins (folic acid), fat-soluble vitamins (vitamin $\mathrm{D}_{2}$ and vitamin $\mathrm{D}_{3}$ ), fatty acids, and botanical marker compounds (ginsenosides) in foods and/or botanical dietary supplement ingredients and finished products.
\end{abstract}

\title{
INTRODUCTION
}

The dietary supplement industry in the US is booming, with three-quarters of adults considering themselves to be supplement users. ${ }^{1}$ Consumption of dietary supplements, which includes vitamin and mineral supplements, represents an annual US expenditure of more than $\$ 40$ billion. These figures represent an increasing American and worldwide trend, and as a result, the verification and maintenance of both the quality and safety of these products is critically important.

The Dietary Supplement Health and Education Act of 1994 (DSHEA) amended the Federal Food, Drug, and Cosmetic Act to create the regulatory category called dietary supplements. The DSHEA also gave the FDA authority to write current Good Manufacturing Practices (cGMPs) that require manufacturers to evaluate the identity, purity, and composition of their ingredients and finished products. In addition, the DSHEA authorized the establishment of the Office of Dietary Supplements at the National Institutes of Health (NIH ODS). To enable members of the dietary supplement community to improve the accuracy of the measurements required for compliance with these and other regulations, NIST established the Dietary Supplements Laboratory Quality Assurance Program (DSQAP) in collaboration with the NIH ODS in 2007.

The program offers the opportunity for laboratories to assess their in-house measurements of active or marker compounds, nutritional elements, contaminants (toxic elements, pesticides, mycotoxins), and fat- and water-soluble vitamins in foods as well as botanical dietary supplement ingredients and finished products. Reports and certificates of participation are provided and can be used to demonstrate compliance with the cGMPs. In addition, NIST and the DSQAP assist the ODS Analytical Methods and Reference Materials program (AMRM) at the NIH in supporting the development and dissemination of analytical tools and reference materials. In the future, results from exercises of the DSQAP and other related programs could be used by ODS to identify problematic matrices and analytes for which an AOAC INTERNATIONAL Official Method of Analysis would benefit the dietary supplement community.

NIST has experience in the administration of quality assurance programs, but the DSQAP takes a unique approach. In other NIST quality assurance programs, a set of analytes is measured repeatedly over time in the same or similar matrices to demonstrate and improve laboratory performance. In contrast, the wide range of matrices and analytes under the "dietary supplements"

\footnotetext{
${ }^{1} 2017$ CRN Consumer Survey on Dietary Supplements. Council for Responsible Nutrition, Washington, DC.
} 
umbrella means that not every laboratory is interested in every sample or analyte. The constantly changing dietary supplement market, and the enormous diversity of finished products, makes repeated determination of a few target compounds in a single matrix of little use to participants. Instead, participating laboratories are interested in testing in-house methods on a wide variety of challenging, real-world matrices to demonstrate that their performance is comparable to that of the community and that their methods provide accurate results. In an area where there are few generally accepted methods, the DSQAP offers a unique tool for assessment of the quality of measurements, provides feedback about performance, and can assist participants in improving laboratory operations.

This report summarizes the results from the fourteenth exercise of the DSQAP, Exercise N. Fiftyfour laboratories responded to the call for participants distributed in May 2017. Samples were shipped to participants in July 2017 and results were returned to NIST by October 2017. This report contains the final data and information that was disseminated to the participants in August 2018.

\section{OVERVIEW OF DATA TREATMENT AND REPRESENTATION}

Individualized data tables and certificates are provided to the participants that have submitted data in each study, in addition to this report. Examples of the data tables using NIST data are also included in each section of this report. Community tables and graphs are provided using randomized laboratory codes, with identities known only to NIST and individual laboratories. The statistical approaches are outlined below for each type of data representation.

\section{$\underline{\text { Statistics }}$}

Data tables and graphs throughout this report contain information about the performance of each laboratory relative to that of the other participants in this study and relative to a target around the expected result, if available. All calculations are performed in PROLab Plus (QuoData GmbH, Dresden, Germany). ${ }^{2}$ The consensus mean and standard deviation are calculated according to the robust algorithm outlined in ISO 13528:2015(E), Annex C. ${ }^{3}$ The algorithm is summarized here in simplified form.

Initial values of the consensus mean, $x^{*}$, and consensus standard deviation, $s^{*}$, are estimated as

$$
\begin{array}{ll}
x^{*}=\text { median of } x_{i} & (i=1,2, \ldots, n) \\
s^{*}=1.483 \times \text { median of }\left|x_{i}-x^{*}\right| & (i=1,2, \ldots, n) .
\end{array}
$$

These initial values for $x^{*}$ and $s^{*}$ are updated by first calculating the expanded standard deviation, $\delta$, as

$$
\delta=1.5 \times s^{*} .
$$

\footnotetext{
${ }^{2}$ Certain commercial equipment, instruments or materials are identified in this certificate to adequately specify the experimental procedure. Such identification does not imply recommendation or endorsement by the National Institute of Standards and Technology, nor does it imply that the materials or equipment identified are necessarily the best available for the purpose.

${ }^{3}$ ISO 13528:2015(E), Statistical methods for use in proficiency testing by interlaboratory comparisons, pp. 53-54.
} 
Each $x_{i}$ is then compared to the expanded range and adjusted to $x_{i}^{*}$ as described below to reduce the effect of outliers.

$$
\begin{aligned}
& \text { If } x_{i}<x^{*}-\delta \text {, then } x_{i}^{*}=x^{*}-\delta \text {. } \\
& \text { If } x_{i}>x^{*}+\delta \text {, then } x_{i}^{*}=x^{*}+\delta \text {. } \\
& \text { Otherwise, } x_{i}^{*}=x_{i} \text {. }
\end{aligned}
$$

New values of $x^{*}, s^{*}$, and $\delta$ are calculated iteratively until the process converges. Convergence is taken as no change from one iteration to the next in the third significant figure of $s^{*}$ and in the equivalent digit in $x^{*}$ :

$$
\begin{aligned}
& x^{*}=\frac{\sum_{i=1}^{n} x_{i}^{*}}{n} \\
& s^{*}=1.134 \times \sqrt{\frac{\sum_{i=1}^{n}\left(x_{i}^{*}-x^{*}\right)}{n-1} .} .
\end{aligned}
$$

Individualized Data Table

The data in this table is individualized to each participating laboratory and is provided to allow participants to directly compare their data to the summary statistics (consensus or community data as well as NIST certified, reference, or estimated values). The upper left of the data table includes the randomized laboratory code. Tables included in this report are generated using NIST data to protect the identity and performance of participants.

Section 1 of the data table contains the laboratory results as reported, including the mean and standard deviation when multiple values were reported. A blank indicates that NIST does not have data on file for that laboratory for a particular analyte or matrix. An empty box for standard deviation indicates that a single value or a value below the limit of quantification (LOQ) for the participant was reported and therefore that value was not included in the calculation of the consensus data. ${ }^{3}$

Also in Section 1 are two Z-scores. The first Z-score, Z' ${ }^{\prime}$ comm, is calculated with respect to the community consensus value, taking into consideration bias that may result from the uncertainty in the assigned consensus value, using $\mathrm{x}^{*}$ and $\mathrm{s}^{*}$ :

$$
Z^{\prime}{ }_{c o m m}=\frac{x_{i}-x *}{\sqrt{2} s *}
$$

The second Z-score, $\mathrm{Z}_{\text {NIST, }}$ is calculated with respect to the target value (NIST certified, reference, or estimated value), using XNIST and $U_{95}$ (the expanded uncertainty) or SNIST (the standard deviation of NIST measurements):

$$
\mathrm{Z}_{\mathrm{NIST}}=\frac{x_{i}-x_{N I S T}}{2 * U_{95}}
$$

or

$$
Z_{N I S T}=\frac{x_{i}-x_{N I S T}}{2 * S_{N I S T}}
$$


The significance of the Z-score and Z'-score is as follows:

- $|Z|<2$ indicates that the laboratory result is considered to be within the community consensus range (for $Z^{\prime}$ comm) or NIST target range (for $Z_{\text {NIST). }}$ )

- $2<|\mathrm{Z}|<3$ indicates that the laboratory result is considered to be marginally different from the community consensus value (for $Z$ ' comm) or NIST target value (for $Z_{\text {NIST). }}$.

- $|Z|>3$ indicates that the laboratory result is considered to be significantly different from the community consensus value (for $Z^{\prime}$ comm) or NIST target value (for $Z_{\text {NIST). }}$ )

Section 2 of the data table contains the community results, including the number of laboratories reporting more than a single value for a given analyte ${ }^{1}$, the mean value determined for each analyte, and a robust estimate of the standard deviation of the reported values. ${ }^{4}$ Consensus means and standard deviations are calculated using the laboratory means; if a laboratory reported a single value or a value below the LOQ, the reported value is not included. ${ }^{3}$ Additional information on calculation of the consensus mean and standard deviation can be found in the previous section.

Section 3 of the data table contains the target values for each analyte. When possible, the target value is a certified or reference value determined at NIST. Certified values and the associated expanded uncertainty ( $\left.U_{95}\right)$ have been determined with two independent analytical methods at NIST, or by combination of a single method at NIST and results from collaborating laboratories. Reference values are assigned using NIST values obtained from the average and standard deviation of measurements made using a single analytical method at NIST or by measurements obtained from collaborating laboratories. For both certified and reference values, at least six samples have been tested and duplicate preparations from the sample package have been included, allowing the uncertainty to encompass variability due to inhomogeneity within and between packages. For samples in which a NIST certified or reference value is not available, the analytes may be measured at NIST using an appropriate method. The NIST-assessed value represents the mean of at least three replicates. For materials acquired from another proficiency testing program, the consensus value and uncertainty from the completed round is used as the target range.

\section{Summary Data Table}

This data table includes a summary of all reported data for a particular analyte in a particular study. Participants can compare the raw data for their laboratory to data reported by the other participating laboratories or to the consensus data. A blank indicates that the laboratory signed up and received samples for that particular analyte and matrix, but NIST does not have data on file for that laboratory.

\section{$\underline{\text { Graphs }}$}

Data Summary View (Method Comparison Data Summary View)

In this view, individual laboratory data are plotted with the individual laboratory standard deviation (error bars). Laboratories reporting values below the method detection limit (MDL) are shown in this view as downward triangles beginning at the LOQ, reported as quantitation limit (QL) on the graphs. Laboratories reporting values as "below QL" can still be successful in the study if the target value is also below the laboratory QL. The solid blue line represents the consensus mean, and the green shaded area represents the $95 \%$ confidence interval for the

${ }^{4}$ ISO 13528:2015(E), Statistical methods for use in proficiency testing by interlaboratory comparisons, Annex C. 
consensus mean, based on the standard error of the consensus mean. The red shaded region represents the target zone for "acceptable" performance, which encompasses the NIST certified, reference, or estimated value bounded by twice its uncertainty $\left(U_{95}\right)$ or twice its standard deviation. The area between the solid red lines represents the range of tolerance (values that result in an acceptable $Z^{\prime}$ score, $\left|Z^{\prime}\right| \leq 2$ ). If the lower limit is below zero, the lower limit has been set to zero. In this view, the relative locations of individual laboratory data and consensus zones with respect to the target zone can be compared easily. In most cases, the target zone and the consensus zone overlap, which is the expected result. Major program goals include reducing the size of the consensus zone and centering the consensus zone about the target value. Analysis of an appropriate reference material as part of a quality control scheme can help to identify sources of bias for laboratories reporting results that are significantly different from the target zone. In the case in which a method comparison is relevant, different colored data points may be used to indicate laboratories that used a specific approach to sample preparation, analysis, or quantitation.

\section{Sample/Sample Comparison View}

In this view, the individual laboratory results for one sample (NIST SRM with a certified, reference, or NIST-determined value) are compared to the results for another sample (another NIST SRM with a more challenging matrix, a commercial sample, etc.). The solid red box represents the target zone for the first sample (x-axis) and the second sample (y-axis). The dotted blue box represents the consensus zone for the first sample (x-axis) and the second sample (y-axis).

The axes of this graph are centered about the consensus mean values for each sample, to a limit of twice the range of tolerance (values that result in an acceptable $Z$ ' score, $\left|Z^{\prime}\right| \leq 2$ ). Depending on the variability in the data, the axes may be scaled proportionally to better display the individual data points for each laboratory. In some cases, when the consensus and target ranges have limited overlap, the solid red box may only appear partially on the graph. If the variability in the data is high (greater than $100 \%$ relative standard deviation (RSD)), the dotted blue box may also only appear partially on the graph. These views emphasize trends in the data that may indicate potential calibration issues or method biases. One program goal is to identify such calibration or method biases and assist participants in improving analytical measurement capabilities. In some cases, when two equally challenging materials are provided, the same view (sample/sample comparison) can be helpful in identifying commonalities or differences in the analysis of the two materials. 


\section{NUTRITIONAL ELEMENTS (Cr) IN MULTIVITAMIN AND CHROMIUM DIETARY SUPPLEMENTS}

Study Overview

In this study, participants were provided with NIST SRM 3280 Multivitamin/Multielement Tablets and candidate SRM 3279 Chromium-Containing Solid Oral Dosage Form. Participants were asked to use in-house analytical methods to determine the mass fraction of chromium in each of the matrices and report values in $\mathrm{mg} / \mathrm{kg}$, on an as-received basis.

Sample Information

Multivitamin. Participants were provided with one bottle containing 30 multivitamin/multielement tablets. Before use, participants were instructed to grind all tablets, mix the resulting powder thoroughly, and to use a sample size of at least $0.2 \mathrm{~g}$. Participants were asked to store the material at controlled room temperature, $10{ }^{\circ} \mathrm{C}$ to $30^{\circ} \mathrm{C}$, and to prepare three samples and report three values from the single bottle provided. The approximate analyte level was not reported to participants prior to the study. The certified value for chromium in SRM 3280 was determined at NIST using instrumental neutron activation analysis (INAA) and X-ray fluorescence spectrometry (XRF). The certified value and uncertainty for chromium is provided in the table below, both on a dry-mass basis and on an as-received basis accounting for moisture of the material (1.37\%).

$$
\text { Certified Mass Fraction in SRM } 3280(\mathrm{mg} / \mathrm{kg})
$$

$\begin{array}{ccc}\stackrel{\text { Analyte }}{\text { Chromium }(\mathrm{Cr})} & \frac{\text { (dry-mass basis) }}{93.7 \pm 2.7} & \frac{\text { (as-received basis) }}{92.4 \pm 2.7}\end{array}$

Chromium Supplement. Participants were provided with three packets, each containing approximately $6 \mathrm{~g}$ of ground chromium supplement. The commercial tablets were ground, homogenized, and heat-sealed inside 4 mil polyethylene bags which were then sealed inside nitrogen-flushed aluminized plastic bags along with two packets of silica gel each. Before use, participants were instructed to thoroughly mix the contents of the packet and to use a sample size of at least $0.25 \mathrm{~g}$. Participants were asked to store the material at controlled room temperature, $10{ }^{\circ} \mathrm{C}$ to $30^{\circ} \mathrm{C}$, and to prepare one sample and report one value from each packet provided. The approximate analyte level was not reported to participants prior to the study. The target value for chromium in candidate SRM 3279 was determined at NIST using inductively coupled plasma mass spectroscopy (ICP-MS). The NIST-determined value and uncertainty for chromium is provided in the table below, on a dry-mass basis and on an as-received basis accounting for moisture of the material $(7.53 \%)$.

NIST-Determined Mass Fraction in Candidate SRM 3279 $(\mathrm{mg} / \mathrm{kg})$

$\begin{array}{lcc}\stackrel{\text { Analyte }}{\text { Chromium }(\mathrm{Cr})} & \frac{\text { (dry-mass basis) }}{1310 \pm 19} & \frac{\text { (as-received basis) }}{1211 \pm 18}\end{array}$




\section{Study Results}

- Forty-nine laboratories enrolled in this exercise and received samples. Thirty-nine laboratories reported results for the chromium supplement ( $80 \%$ participation) and 37 laboratories reported results for the multivitamin/multielement tablets ( $76 \%$ participation).

- The consensus mean for chromium in the chromium supplement was below the target range, while the consensus mean for chromium in the multivitamin was within the target range.

- The between-laboratory variability was very good for both the chromium supplement and the multivitamin (5\% RSD and $12 \%$ RSD, respectively).

- All but one of the laboratories reported using ICP-MS (97\%) as their analytical method for measuring chromium. The remaining laboratory did not specify a method (3\%).

\section{Technical Recommendations}

The following recommendations are based on results obtained from the participants in this study.

- As shown in Figure 3, many laboratories reported data for chromium in the multivitamin sample that was within the NIST target range for the but was lower than the NIST target range for the chromium supplement.

- Laboratories that reported the correct value for the multivitamin but a low value for the chromium supplement may have experienced greater difficulty in digestion of the chromium supplement compared with the multivitamin.

- While sample preparation information from the participants was limited, the use of a small amount of $\mathrm{HF}$ and an increase in temperature may be needed for complete digestions required for analysis.

- A matrix interference may be present in either one or both samples. The use of standard additions may reduce the impact of matrix interferences.

- A linear calibration curve that surrounds the expected sample concentration values should be used for calculation. This curve should include both the lowest and highest expected concentration values of the sample solutions. Extrapolation of results beyond calibration curves may result in the low values seen in the chromium supplement.

- Most laboratories had good sample-to-sample variability (<6\% for multivitamins and $<13 \%$ for chromium supplement). Difficulty in the digestion of samples will cause increased variability between samples, which may explain the greater variability observed for the chromium supplement.

- For both the multivitamin and the chromium supplement, a few laboratories reported data significantly outside of the target and consensus ranges. The use of appropriate quality assurance samples to establish that a method is in control and performing correctly may reduce the likelihood of outlying data. Quality assurance samples can be commercially available reference materials (CRMs, SRMs, or RMs) or prepared in-house.

- All results should be checked closely to avoid calculation errors and to be sure that results are reported in the requested units. 
Table 1. Individualized data summary table (NIST) for chromium in multivitamin and chromium supplement.

\section{National Institute of Standards \& Technology}

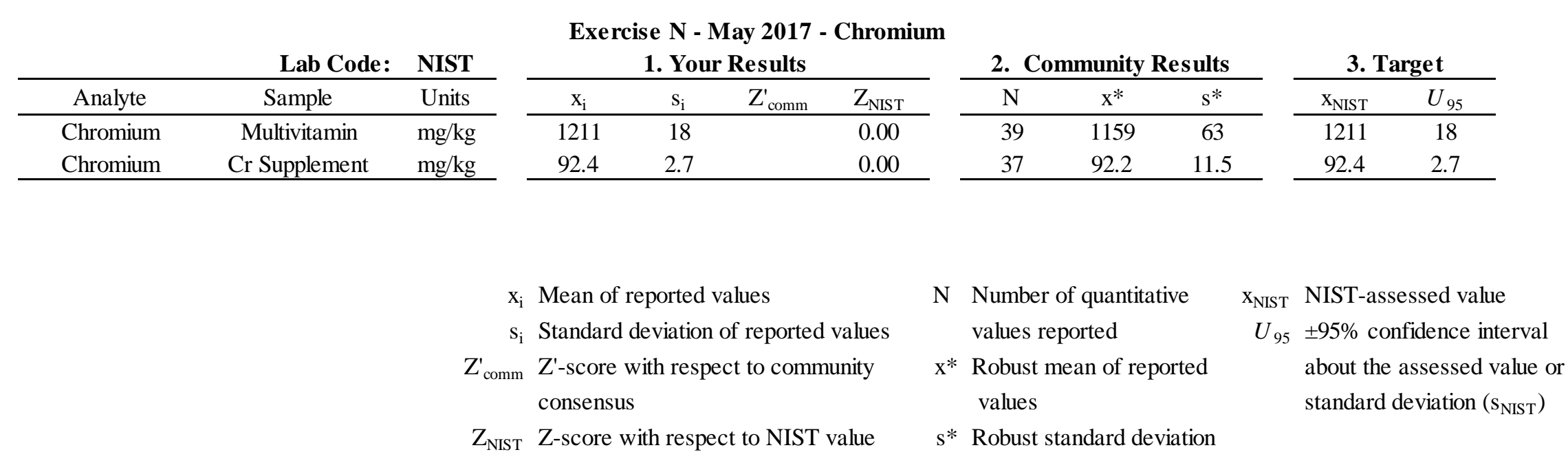


Table 2. Data summary table for chromium in multivitamin and a chromium supplement. Data points highlighted in red have been flagged as potential outliers (e.g., difference from reference value, Grubb and/or Cochran) by the NIST software package.

\begin{tabular}{|c|c|c|c|c|c|c|c|c|c|c|c|}
\hline & \multirow[b]{3}{*}{ Lab } & \multicolumn{10}{|c|}{ Chromium } \\
\hline & & \multicolumn{5}{|c|}{ SRM 3279 Chromium-Containing Solid Oral Dosage Form (mg/kg) } & \multicolumn{5}{|c|}{ SRM 3280 Multivitamin/Multielement Tablets (mg/kg) } \\
\hline & & A & B & C & Avg & SD & A & B & $\mathbf{C}$ & Avg & SD \\
\hline \multirow{50}{*}{ 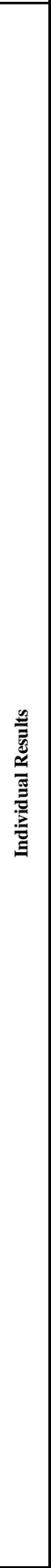 } & NIST & & & & 1211 & 18 & & & & 92.4 & 2.7 \\
\hline & N102 & 1235 & 1164 & 1157 & 1185 & 43 & 94.6 & 94.7 & 94.6 & 94.6 & 0.1 \\
\hline & N103 & 1142 & 1115 & 1198 & 1152 & 42 & 94.0 & 91.0 & 87.0 & 90.7 & 3.5 \\
\hline & N104 & 1170 & 1180 & 1180 & 1177 & 6 & 88.6 & 82.0 & 77.3 & 82.6 & 5.7 \\
\hline & N105 & 1156 & 1048 & 1070 & 1091 & 57 & 104.0 & 94.0 & 84.0 & 94.0 & 10.0 \\
\hline & N106 & 1170 & 1200 & 1210 & 1193 & 21 & 103.0 & 104.0 & 100.0 & 102.3 & 2.1 \\
\hline & N107 & 1232 & 1264 & 1211 & 1236 & 26 & 86.0 & 86.4 & 87.8 & 86.7 & 1.0 \\
\hline & N108 & 1276 & 1249 & 1238 & 1254 & 20 & 87.9 & 86.7 & 89.1 & 87.9 & 1.2 \\
\hline & N110 & 1201 & 1244 & 1248 & 1231 & 26 & 102.0 & 98.7 & 87.5 & 96.1 & 7.6 \\
\hline & N111 & 1137 & 1093 & 1120 & 1117 & 22 & 113.0 & 137.0 & 140.0 & 130.0 & 14.8 \\
\hline & N112 & 1274 & 1278 & 1247 & 1266 & 17 & 105.0 & 109.0 & 107.0 & 107.0 & 2.0 \\
\hline & N113 & 1.20 & 1.18 & 1.21 & 1.20 & 0.02 & 0.094 & 0.10 & 0.09 & 0.092 & 0.006 \\
\hline & N114 & 1090 & 1130 & 1170 & 1130 & 40 & 73.9 & 77.8 & 92.1 & 81.3 & 9.6 \\
\hline & N115 & & & & & & & & & & \\
\hline & N116 & 1172 & 1177 & 1191 & 1180 & 10 & 100.4 & 100.1 & 101.9 & 100.8 & 0.9 \\
\hline & N119 & 1160 & 1186 & 1144 & 1163 & 21 & 95.6 & 104.3 & 97.6 & 99.2 & 4.6 \\
\hline & N120 & & & & & & & & & & \\
\hline & N121 & 1161 & 1162 & 1139 & 1154 & 13 & 99.3 & 96.6 & 95.6 & 97.2 & 1.9 \\
\hline & N122 & 1142 & 1115 & 1198 & 1152 & 42 & 94.0 & 91.0 & 87.0 & 90.7 & 3.5 \\
\hline & N123 & 1.13 & 1.10 & 1.14 & 1.12 & 0.02 & 0.082 & 0.092 & 0.087 & 0.087 & 0.005 \\
\hline & N124 & 1125 & 1132 & 1132 & 1130 & 4 & 96.6 & 96.0 & 111.0 & 101.2 & 8.5 \\
\hline & N125 & & & & & & & & & & \\
\hline & N126 & 1099 & 1140 & 1142 & 1127 & 24 & 89.0 & 94.8 & 78.5 & 87.4 & 8.3 \\
\hline & N127 & 1156 & 1098 & 1132 & 1129 & 29 & 94.8 & 92.7 & 91.2 & 92.9 & 1.8 \\
\hline & N129 & 1188 & 1192 & 1186 & 1189 & 3 & 89.8 & 89.2 & 90.5 & 89.8 & 0.7 \\
\hline & N130 & 1147 & 1163 & 1135 & 1148 & 14 & 82.7 & 93.2 & 87.7 & 87.9 & 5.3 \\
\hline & N131 & & & & & & & & & & \\
\hline & N132 & 1070 & 1130 & 1180 & 1127 & 55 & 93.8 & 96.7 & 98.6 & 96.4 & 2.4 \\
\hline & N133 & 986 & 970 & 998 & 985 & 14 & 72.2 & 73.7 & 77.3 & 74.4 & 2.6 \\
\hline & N134 & 1158 & 1176 & 1190 & 1175 & 16 & 75.7 & 76.9 & 79.3 & 77.3 & 1.8 \\
\hline & N138 & 1080 & 1090 & 1110 & 1093 & 15 & & & & & \\
\hline & N139 & 1049 & 1058 & 1073 & 1060 & 12 & 92.7 & 94.2 & 98.6 & 95.1 & 3.0 \\
\hline & N140 & 1336 & 1333 & 1339 & 1336 & 3 & 92.8 & 93.6 & 91.4 & 92.6 & 1.1 \\
\hline & N141 & & & & & & & & & & \\
\hline & $\mathrm{N} 142$ & 1304 & 1332 & 1352 & 1330 & 24 & 118.1 & 94.7 & 106.8 & 106.5 & 11.7 \\
\hline & N144 & & & & & & & & & & \\
\hline & N145 & 1150 & 1110 & 1160 & 1140 & 26 & 103.0 & 91.0 & 90.7 & 94.9 & 7.0 \\
\hline & N146 & & & & & & & & & & \\
\hline & N147 & & & & & & & & & & \\
\hline & N148 & 2097 & 1470 & 1025 & 1530 & 539 & 95.2 & 94.2 & 94.1 & 94.5 & 0.6 \\
\hline & N149 & 1190 & 1210 & 1180 & 1193 & 15 & 111.0 & 90.4 & 88.6 & 96.7 & 12.4 \\
\hline & N150 & & & & & & & & & & \\
\hline & N151 & & & & & & & & & & \\
\hline & N152 & 1172 & 1167 & 1167 & 1169 & 3 & 103.4 & 103.1 & 84.0 & 96.8 & 11.1 \\
\hline & N153 & 1130 & 1088 & 1126 & 1115 & 23 & 88.3 & 80.5 & 78.9 & 82.6 & 5.0 \\
\hline & N154 & 1101 & 1151 & 1234 & 1162 & 67 & 79.8 & & & 79.8 & \\
\hline & N155 & 1170 & 1160 & 1130 & 1153 & 21 & 92.8 & 91.5 & 90.5 & 91.6 & 1.2 \\
\hline & N156 & 1190 & 1140 & 1130 & 1153 & 32 & 74.3 & 72.4 & 68.2 & 71.6 & 3.1 \\
\hline & N157 & 1185 & 1215 & 1157 & 1185 & 29 & 115.6 & 109.8 & 100.4 & 108.6 & 7.7 \\
\hline & N158 & 1147 & 1127 & 1131 & 1135 & 11 & 108.4 & 108.4 & 110.3 & 109.0 & 1.1 \\
\hline \multirow{5}{*}{ 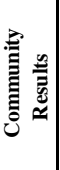 } & & \multicolumn{3}{|c|}{ Consensus Mean } & \multicolumn{2}{|l|}{1159} & \multicolumn{2}{|c|}{ Consensus Mean } & & 92.2 & \\
\hline & & \multicolumn{3}{|c|}{ Consensus Standard Deviation } & \multicolumn{2}{|l|}{63} & \multicolumn{3}{|c|}{ Consensus Standard Deviation } & 11.5 & \\
\hline & & & \multicolumn{2}{|l|}{1530} & Maximum & & & 130.0 & \\
\hline & & Minimum & & & 1.12 & & Minimum & & & 0.087 & \\
\hline & & $\mathrm{N}$ & & & 39 & & $\mathrm{~N}$ & & & 37 & \\
\hline
\end{tabular}




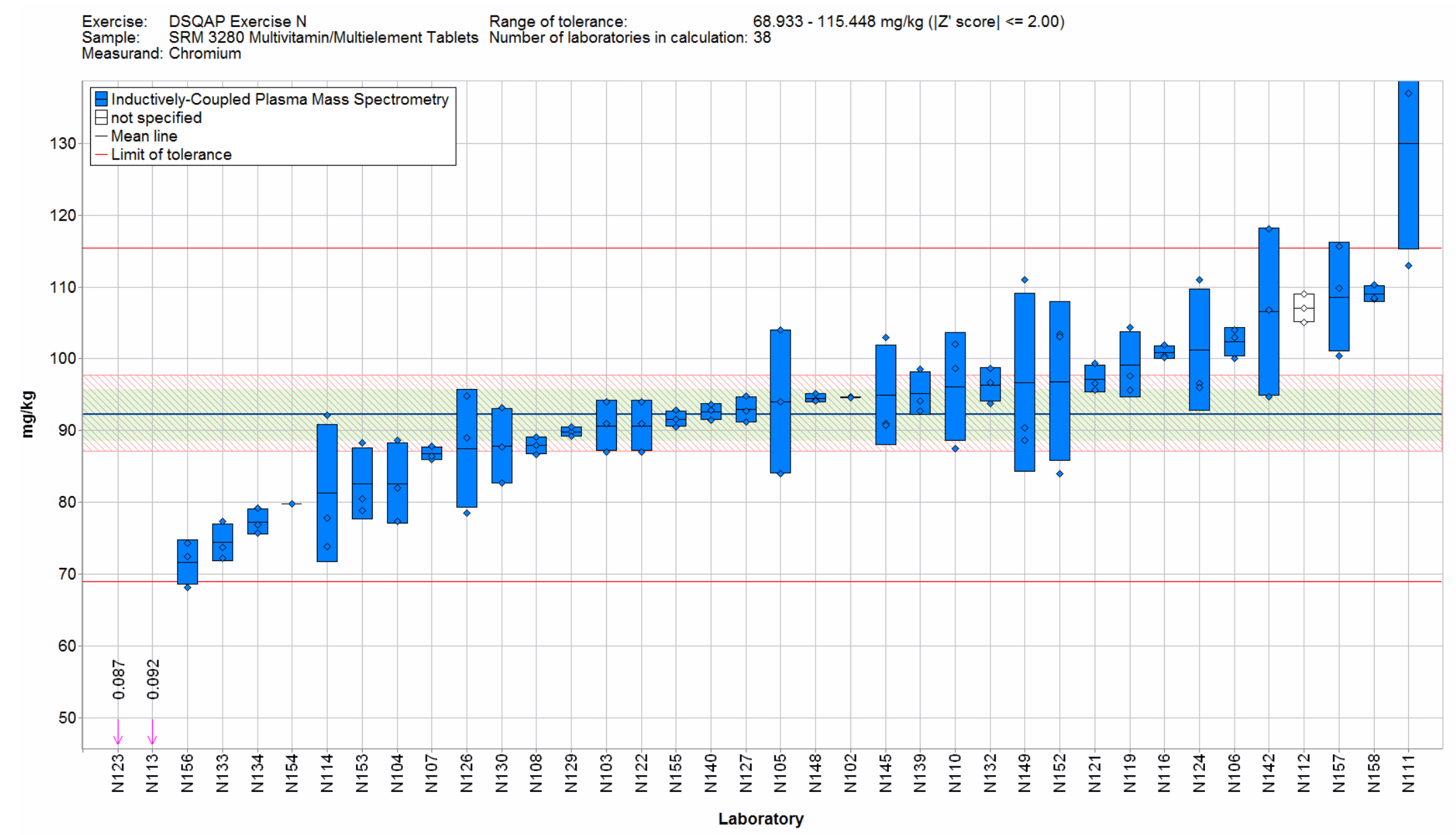

Figure 1. Chromium in SRM 3280 Multivitamin/Multielement Tablets (data summary view - analytical method). In this view, individual laboratory data are plotted (diamonds) with the individual laboratory standard deviation (rectangle). The color of the data point represents the analytical method employed. The solid blue line represents the consensus mean and the green shaded region represents the $95 \%$ confidence interval for the consensus mean. The solid red lines represent the consensus range of tolerance, calculated as the values above and below the consensus mean that result in an acceptable $Z_{\text {comm }}^{\prime}$ score, $\left|Z_{\text {comm }}^{\prime}\right| \leq 2$. The red shaded region represents the NIST range of tolerance, which encompasses the NIST certified value bounded by twice its uncertainty (U95) and represents the range that results in an acceptable $Z_{\text {NIST }}$ score, $\left|Z_{\text {NIST }}\right| \leq 2$. 


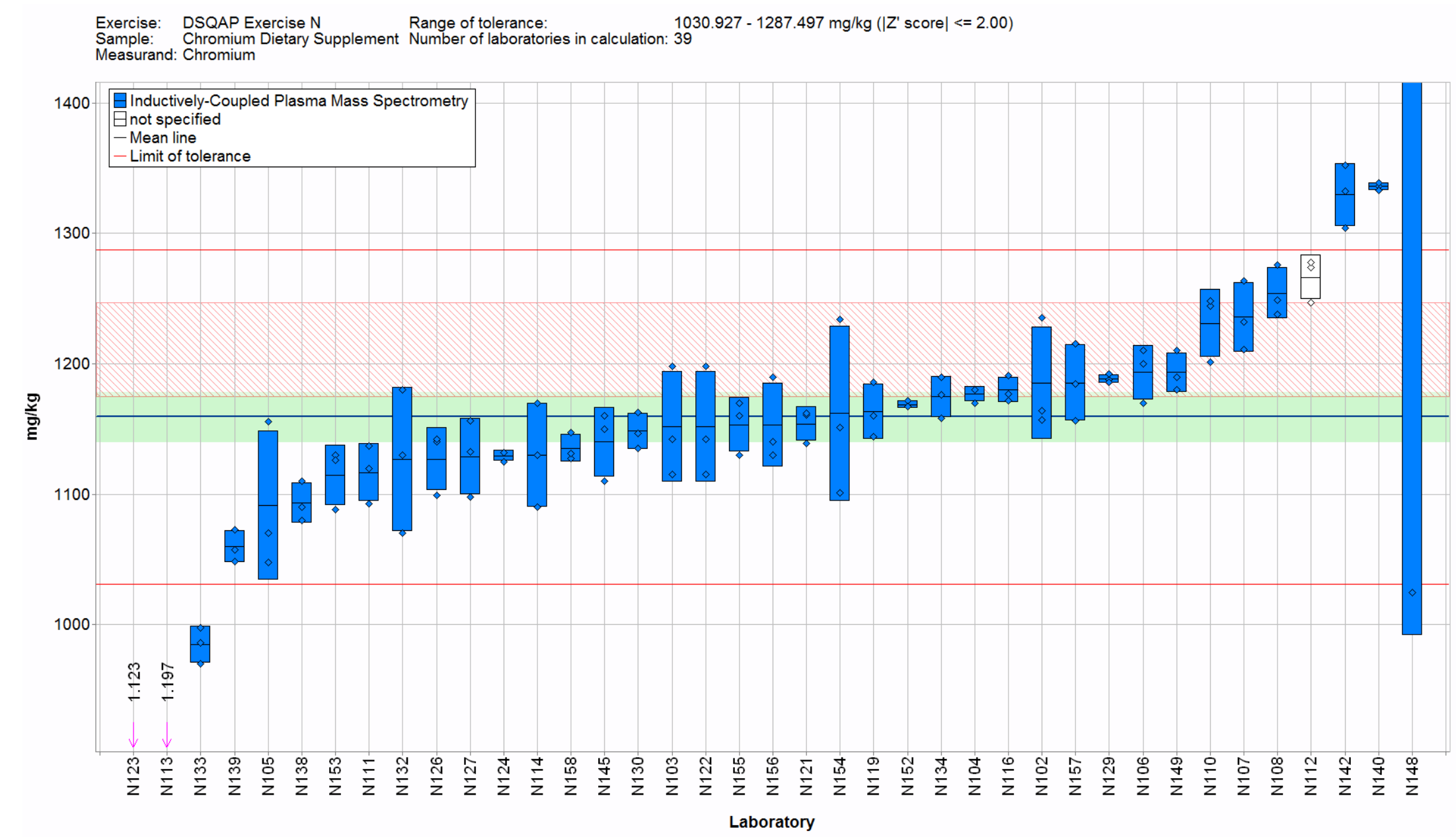

Figure 2. Chromium in candidate SRM 3279 Chromium-Containing Solid Oral Dosage Form (data summary view - analytical method). In this view, individual laboratory data are plotted (diamonds) with the individual laboratory standard deviation (rectangle). The color of the data point represents the analytical method employed. The solid blue line represents the consensus mean and the green shaded region represents the $95 \%$ confidence interval for the consensus mean. The solid red lines represent the consensus range of tolerance, calculated as the values above and below the consensus mean that result in an acceptable $Z_{\text {comm }}^{\prime}$ score, $\left|Z_{\text {comm }}^{\prime}\right| \leq 2$. The red shaded region represents the NIST range of tolerance, which encompasses the NIST-determined value bounded by twice its uncertainty ( $U_{95}$ ) and represents the range that results in an acceptable $Z_{\text {NIST }}$ score, $\left|Z_{\text {NIST }}\right| \leq 2$. 


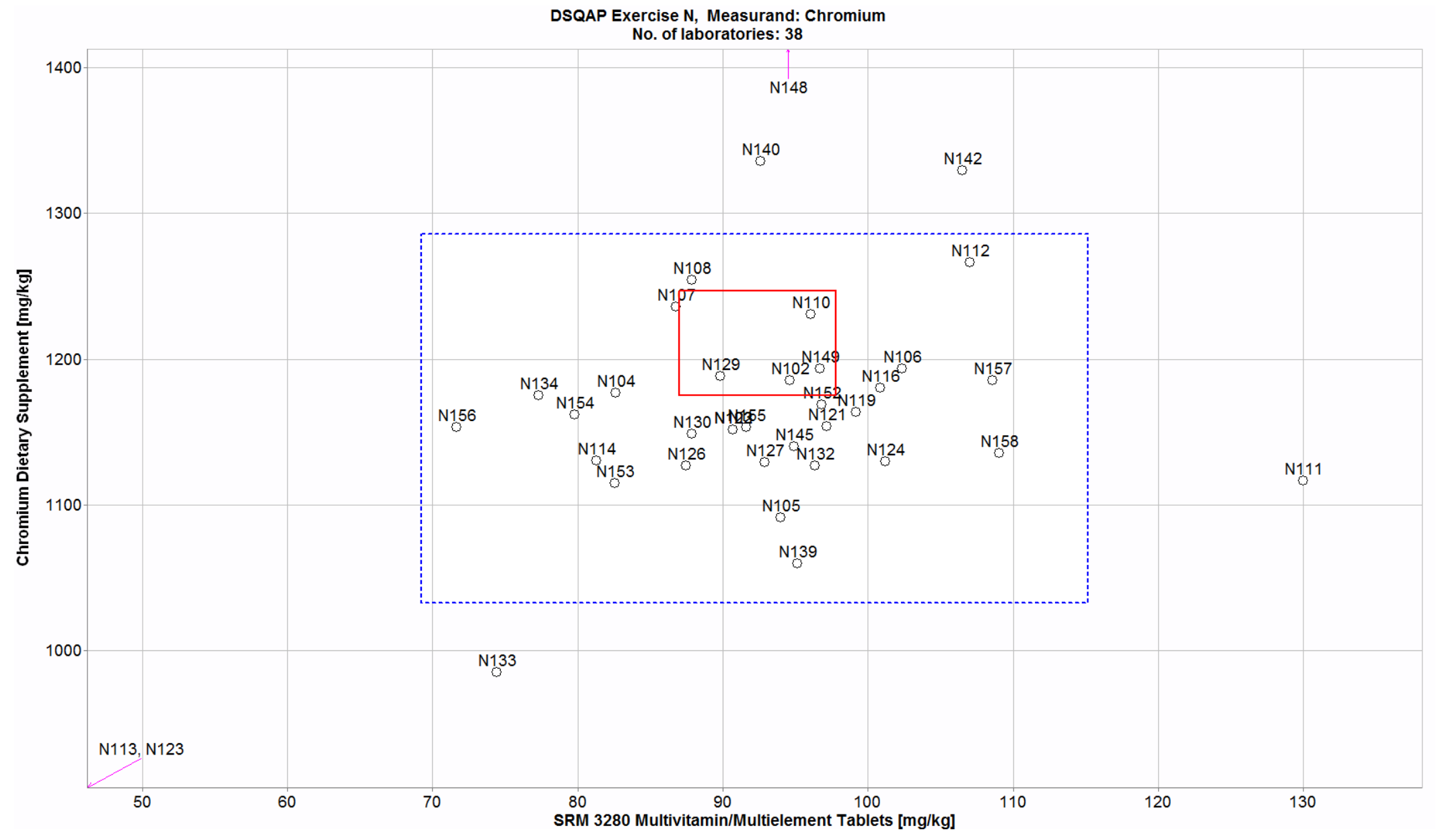

Figure 3. Laboratory means for chromium in SRM 3280 Multivitamin/Multielement Tablets and candidate SRM 3279 Chromium-Containing Solid Oral Dosage Form (sample/sample comparison view). In this view, the individual laboratory mean for one sample (multivitamin) is compared to the mean for a second sample (chromium supplement). The solid red box represents the NIST range of tolerance for the two samples, multivitamin (x-axis) and chromium supplement (y-axis), which encompasses the NIST values bounded by their uncertainties $\left(U_{95}\right)$ and represents the range that results in an acceptable $Z_{\text {NIST }}$ score, $\left|Z_{\text {NIST }}\right| \leq 2$. The dotted blue box represents the consensus range of tolerance for multivitamin (x-axis) and chromium supplement (y-axis), calculated as the values above and below the consensus means that result in an acceptable $Z_{\text {comm }}^{\prime}$ score, $\left|Z_{\text {comm }}^{\prime}\right| \leq 2$. 


\section{TOXIC ELEMENTS (As, Cd, AND Pb) IN CALCIUM AND GINKGO DIETARY SUPPLEMENTS}

\section{Study Overview}

In this study, participants were provided with two NIST SRMs, SRM 3532 Calcium-Containing Solid Oral Dosage Form and SRM 3248 Ginkgo-Containing Tablets. Participants were asked to use in-house analytical methods to determine the mass fractions of arsenic (As), cadmium (Cd), and lead $(\mathrm{Pb})$ in each of the matrices and report values in $\mathrm{ng} / \mathrm{g}$, on an as-received basis.

\section{$\underline{\text { Sample Information }}$}

Calcium Supplement. Participants were provided with three packets, each containing approximately $10 \mathrm{~g}$ of ground calcium supplement. The commercial tablets were ground, homogenized, and heat-sealed inside 4 mil polyethylene bags, which were then sealed inside nitrogen-flushed aluminized plastic bags along with two packets of silica gel. Before use, participants were instructed to thoroughly mix the contents of each packet and use a sample size of at least $0.75 \mathrm{~g}$. Participants were asked to store the material at controlled room temperature, $10{ }^{\circ} \mathrm{C}$ to $30{ }^{\circ} \mathrm{C}$, and to report a single value from each packet provided. Approximate analyte levels were not reported to participants prior to the study. The NIST certified value for cadmium in SRM 3532 was determined at NIST by using isotope dilution inductively coupled plasma mass spectrometry (ID ICP-MS). The NIST reference value for lead in SRM 3532 was also determined at NIST by using ID ICP-MS. The NIST-determined value for arsenic in SRM 3532 was estimated at NIST by using ICP-MS and INAA. The NIST values and uncertainties for $\mathrm{As}, \mathrm{Cd}$, and $\mathrm{Pb}$ are provided in the table below, both on an as-received basis and on a dry-mass basis, accounting for moisture of the material $(3.2 \%)$.

NIST-Determined Mass Fraction in SRM 3532 (ng/g)

Analyte
Arsenic $(\mathrm{As})$
Cadmium $(\mathrm{Cd})$
Lead $(\mathrm{Pb})$

(dry-mass basis)
$365 \pm 94$
$97.9 \pm 1.2$
$225 \pm 33$

(as-received basis) $353 \pm 91$$$
94.8 \pm 1.2
$$$$
218 \pm 31
$$

Ginkgo Supplement. Participants were provided with three packets, each containing approximately $1 \mathrm{~g}$ of ground Ginkgo supplement. The commercial tablets were ground, homogenized, and heat-sealed inside 4 mil polyethylene bags which were then sealed inside nitrogen-flushed aluminized plastic bags along with two packets of silica gel each. Before use, participants were instructed to thoroughly mix the contents of the packet and to use a sample size of at least $0.75 \mathrm{~g}$. Participants were asked to store the material at controlled room temperature, $10{ }^{\circ} \mathrm{C}$ to $30{ }^{\circ} \mathrm{C}$, and to prepare one sample and report one value from each packet provided. The approximate analyte levels were not reported to participants prior to the study. The NIST certified value for lead in SRM 3248 was determined at NIST and at the National Research Council Canada (NRCC) using ID ICP-MS. The NIST reference values for arsenic and cadmium were determined at NIST by INAA and ID ICP-MS, respectively. The NIST values and uncertainties for As, $\mathrm{Cd}$, and $\mathrm{Pb}$ are provided in the table below, on a dry-mass basis and on an as-received basis accounting for moisture of the material $(4.78 \%)$. 
NIST-Determined Mass Fraction in SRM 3248 (ng/g)

$\underline{\text { Analyte }}$
Arsenic $(\mathrm{As})$
Cadmium $(\mathrm{Cd})$
Lead $(\mathrm{Pb})$

(dry-mass basis)

\begin{tabular}{|c|c|c|}
\hline 56.5 & & \\
\hline 1.56 & & 0.19 \\
\hline 75.3 & & 8.5 \\
\hline
\end{tabular}

(as-received basis)

\begin{tabular}{|c|c|c|}
\hline 53.8 & + & 4.1 \\
\hline 1.49 & & 0.18 \\
\hline 8.2 & & 8.5 \\
\hline
\end{tabular}

$\underline{\text { Study Results }}$

- Forty-seven laboratories enrolled in the exercise and received samples to measure arsenic in both supplements. Thirty-three laboratories reported results for arsenic in the calcium supplement (70\% participation). Thirty-two laboratories reported results for arsenic in the ginkgo supplement (68\% participation).

- The consensus means for arsenic in both materials were within the NIST target ranges. The between-laboratory variability was high for both the calcium supplement and the ginkgo supplement (35\% RSD and $42 \% \mathrm{RSD}$, respectively).

- All but one of the laboratories reported using ICP-MS (97\%) as their analytical method for measuring arsenic. The remaining laboratory did not specify a method (3\%).

- Forty-nine laboratories enrolled in the exercise and received samples to measure cadmium in both supplements. Thirty-six laboratories reported results for cadmium in the calcium supplement (73\% participation). Sixteen laboratories reported results for cadmium in the ginkgo supplement (33\% participation).

- The consensus mean for cadmium was below the target range of the calcium supplement with good between-laboratory variability (19\% RSD).

- The consensus mean for cadmium was above the target range of the ginkgo supplement with very high between-laboratory variability (65\% RSD).

- All but one of the laboratories reported using ICP-MS (97\%) as their analytical method for measuring cadmium. The remaining laboratory did not specify a method (3\%).

- Forty-nine laboratories enrolled in the exercise and received samples to measure lead in both supplements. Thirty-seven laboratories reported results for lead in the calcium supplement (76\% participation). Thirty-six laboratories reported results for lead in the ginkgo supplement (73\% participation).

- The consensus mean for lead was within the target range of the calcium supplement with good between-laboratory variability ( $23 \% \mathrm{RSD})$.

- The consensus mean for lead was below the target range of the ginkgo supplement, but the consensus range slightly overlapped the target range. The between-laboratory variability was very good for lead in the ginkgo supplement (10\% RSD).

- All but one laboratory reported using ICP-MS (97\%) as their analytical method for measuring arsenic. The remaining laboratory did not specify a method (3\%). 
Technical Recommendations

The following recommendations are based on results obtained from the participants in this study.

- Difficulty in the digestion of samples will cause increased variability between samples. Supplements and tablets can be hard to digest, requiring higher temperatures or the use of a small amount of HF to ensure complete digestion of the materials.

- The high level of $\mathrm{Ca}$ in the calcium supplement can cause a buildup on the ICP-MS cones if sample solutions are not diluted. Unfortunately, the analyte of interest is also diluted, and sensitivity is decreased for analytes that are already low in the matrix.

- A difference in reporting units among laboratories is the suspected reason for the occurrence of high between-laboratory variability. Calculation errors may be a cause for incorrect results. Using a quality assurance material (CRM, SRM, RM), or in-house prepared material, to establish that a method is in control will also help find calculation errors. Once a method and quality assurance material appear to be in control, be sure results are reported in the correct units.

- Arsenic is volatile and can be lost during sample preparation. For laboratories experiencing low arsenic values the following may be of help;

- High temperatures using a vigorous microwave digestion should convert all volatile organoarsenic species to arsenic acid (AsV), at which point subsequent heating will not result in loss of arsenic. Open-beaker digestions should not be used for As analysis and closed-vessel digestions should be opened with care ensuring that no As is lost as a result of inadvertent venting.

- Extrapolation of samples lying above the calibration curve may result in incorrect results. Ensure calibration curves are linear at the point where sample concentrations are expected to be measured.

- Spectral interferences can make cadmium difficult to measure accurately by ICP-MS, which may be the cause of some of the high QLs reported in the data tables.

- A scan of the sample before analysis will indicate any potential interferences in the sample that will need to be addressed. High concentrations of certain elements, mainly Mo, Sn, or $\mathrm{Zr}$, are known to cause interferences in the analysis of cadmium by ICP-MS.

- The high level of $\mathrm{Ca}$ compared to $\mathrm{Cd}$ in the calcium dietary supplement may also cause interference, especially when using ${ }^{112} \mathrm{Cd}\left({ }^{40} \mathrm{Ca}_{2}{ }^{16} \mathrm{O}\right)_{2}$.

- Examples of molecular interferences include ${ }^{95,96,97 \text { and } 98} \mathrm{Mo}^{16} \mathrm{O}^{+},{ }^{94,95,96, \text { and } 97} \mathrm{Mo}^{16} \mathrm{O}^{1} \mathrm{H}^{+}$, ${ }^{96} \mathrm{Zr}^{16} \mathrm{O}^{+},{ }^{94}$ and ${ }^{96} \mathrm{Zr}^{16} \mathrm{O}^{1} \mathrm{H}^{+},{ }^{40} \mathrm{Ar}_{2}{ }^{16} \mathrm{O}_{2},{ }^{40} \mathrm{Ca}_{2}{ }^{16} \mathrm{O}_{2}$, or ${ }^{40} \mathrm{Ca}_{2}{ }^{16} \mathrm{O}_{2}{ }^{1} \mathrm{H}^{+}$. Examples of elemental isobaric interferences include ${ }^{112} \mathrm{Sn},{ }^{113} \mathrm{In}$, and ${ }^{114} \mathrm{Sn}$.

- Anion chromatography prior to ICP-MS can reduce interferences. If this is not practical, collision cell technology can be used to remove many of the molecular interferences that may be found in these two materials.

- Analysis of an appropriate number of procedural blanks is always important and can be critical when sample concentrations are near the detection limit, as with cadmium in the ginkgo supplement.

- Lead is easily digested and volatile loss of $\mathrm{Pb}$ is not a concern. Digestion with $\mathrm{HCl}$ may form a highly insoluble $\mathrm{PbCl}_{2}$ precipitate, so digestion with $\mathrm{HNO}_{3}$ is recommended.

- Some laboratories had high sample-to-sample variability (20\% to $>50 \%$ ), which may be caused by incomplete sample digestion, matrix interferences, or calibration curves which do not encompass all sample solutions measured. 
Calibration curves must be linear and include the lowest and highest values expected to be measured in the sample solutions. Extrapolation of the curve may cause incorrect results. 
Table 3. Individualized data summary table (NIST) for arsenic, cadmium, and lead in calcium and ginkgo supplements.

\section{National Institute of Standards \& Technology}

\begin{tabular}{|c|c|c|c|c|c|c|c|c|c|c|c|}
\hline \multirow[b]{3}{*}{ Analyte } & \multirow{3}{*}{$\begin{array}{l}\text { Lab Code: } \\
\text { Sample } \\
\end{array}$} & \multirow{3}{*}{$\begin{array}{c}\text { NIST } \\
\text { Units }\end{array}$} & \multicolumn{7}{|c|}{ Exercise N - May 2017 - Toxic Elements } & \multirow{2}{*}{\multicolumn{2}{|c|}{ 3. Target }} \\
\hline & & & \multicolumn{4}{|c|}{ 1. Your Results } & \multicolumn{3}{|c|}{ 2. Community Results } & & \\
\hline & & & $x_{i}$ & $\mathrm{~s}_{\mathrm{i}}$ & $\mathrm{Z}_{\text {comm }}^{\prime}$ & $\mathrm{Z}_{\mathrm{NIST}}$ & $\mathrm{N}$ & $\mathrm{x}^{*}$ & $\mathrm{~s}^{*}$ & $\mathrm{x}_{\mathrm{NIST}}$ & $U_{95}$ \\
\hline Arsenic & Ca Supplement & $\mathrm{ng} / \mathrm{g}$ & 353 & 91 & & 0.00 & 33 & 324 & 114 & 353 & 91 \\
\hline Arsenic & Ginkgo Tablets & $\mathrm{ng} / \mathrm{g}$ & 54.0 & 4.1 & & 0.00 & 32 & 58.2 & 24.4 & 54.0 & 4.1 \\
\hline Cadmium & Ca Supplement & $\mathrm{ng} / \mathrm{g}$ & 94.8 & 1.2 & & 0.00 & 36 & 85 & 16 & 94.8 & 1 \\
\hline Cadmium & Ginkgo Tablets & $\mathrm{ng} / \mathrm{g}$ & 1.49 & 0.18 & & 0.00 & 17 & 3 & 2 & 1.5 & 0.2 \\
\hline Lead & Ca Supplement & $\mathrm{ng} / \mathrm{g}$ & 218 & 31 & & 0.00 & 37 & 223 & 51 & 218 & 31 \\
\hline Lead & Ginkgo Tablets & $\mathrm{ng} / \mathrm{g}$ & 738 & 9 & & 0.00 & 36 & 712 & 71 & 738 & 9 \\
\hline
\end{tabular}

\footnotetext{
$\mathrm{x}_{\mathrm{i}}$ Mean of reported values

$s_{i}$ Standard deviation of reported values

$Z_{\text {comm }}^{\prime} Z^{\prime}$-score with respect to community consensus

$\mathrm{Z}_{\mathrm{NIST}} \mathrm{Z}$-score with respect to NIST value
}

$\mathrm{N}$ Number of quantitative values reported

$\mathrm{x}^{*}$ Robust mean of reported values

s* Robust standard deviation $\mathrm{x}_{\text {NIST }}$ NIST-assessed value

$U_{95} \pm 95 \%$ confidence interval about the assessed value or standard deviation $\left(\mathrm{s}_{\mathrm{NIST}}\right)$ 
Table 4. Data summary table for total arsenic in calcium and ginkgo supplements. Data highlighted in red have been flagged as potential outliers (e.g., difference from reference value, Grubb and/or Cochran) by the NIST software package.

\begin{tabular}{|c|c|c|c|c|c|c|c|c|c|c|c|}
\hline & \multirow[b]{3}{*}{ Lab } & \multicolumn{10}{|c|}{ Total Arsenic } \\
\hline & & \multicolumn{5}{|c|}{ SRM 3532 Calcium Dietary Supplement (ng/g) } & \multicolumn{5}{|c|}{ SRM 3248 Ginkgo-Containing Tablets (ng/g) } \\
\hline & & $\mathbf{A}$ & $\mathbf{B}$ & $\mathbf{C}$ & Avg & SD & $\mathbf{A}$ & $\mathbf{B}$ & $\mathbf{C}$ & Avg & SD \\
\hline \multirow{48}{*}{ 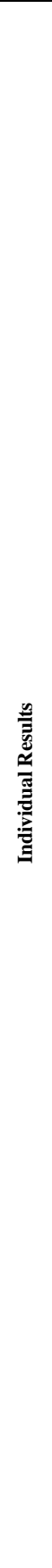 } & NIST & & & & 353 & 91 & & & & 53.8 & 4.1 \\
\hline & N102 & 271 & 261 & 265 & 266 & 5 & 44.0 & 40.0 & 47.0 & 43.7 & 3.5 \\
\hline & N103 & 313 & 276 & 289 & 293 & 19 & 131.0 & 147.0 & 150.0 & 142.7 & 10.2 \\
\hline & N104 & 312 & 310 & 318 & 313 & 4 & 46.7 & 46.7 & 49.3 & 47.6 & 1.5 \\
\hline & N105 & 317 & 264 & 285 & 289 & 27 & $<50$ & $<50$ & $<50$ & $<50$ & \\
\hline & N106 & 370 & 370 & 390 & 377 & 12 & 56.0 & 47.0 & 58.0 & 53.7 & 5.9 \\
\hline & N107 & 70 & 74 & 74 & 73 & 3 & 30.2 & 28.0 & 27.7 & 28.6 & 1.4 \\
\hline & N108 & 309 & 329 & 302 & 313 & 14 & 52.0 & 53.0 & 52.0 & 52.3 & 0.6 \\
\hline & N110 & 706 & 722 & 716 & 714 & 8 & 83.6 & 90.7 & 85.8 & 86.7 & 3.6 \\
\hline & N111 & 204 & 228 & 321 & 251 & 62 & 102.0 & 102.0 & 101.0 & 101.7 & 0.6 \\
\hline & N112 & 276 & 339 & 296 & 304 & 32 & 72.0 & 69.0 & 69.0 & 70.0 & 1.7 \\
\hline & $\mathrm{N} 113$ & 292 & 275 & 252 & 273 & 20 & 51.0 & 71.0 & 60.8 & 60.9 & 10.0 \\
\hline & N114 & 356 & 358 & 913 & 542 & 321 & 63.0 & 45.0 & 55.0 & 54.3 & 9.0 \\
\hline & N115 & & & & & & & & & & \\
\hline & N116 & & & & & & & & & & \\
\hline & N118 & 172 & 178 & 203 & 184 & 16 & 26.5 & 27.6 & 23.7 & 25.9 & 2.0 \\
\hline & N119 & 297 & 304 & 292 & 298 & 6 & 48.8 & 46.9 & 47.6 & 47.8 & 1.0 \\
\hline & N120 & & & & & & & & & & \\
\hline & N121 & 0.310 & 0.350 & 0.340 & 0.333 & 0.021 & 0.050 & 0.050 & 0.050 & 0.050 & 0.000 \\
\hline & N122 & 313 & 276 & 289 & 293 & 19 & 131.0 & 147.0 & 150.0 & 142.7 & 10.2 \\
\hline & N124 & 390 & 380 & 394 & 388 & 8 & 48.5 & 50.0 & 53.6 & 50.7 & 2.6 \\
\hline & N125 & & & & & & & & & & \\
\hline & N126 & 372 & 371 & 374 & 372 & 2 & 59.0 & 59.0 & 55.0 & 57.7 & 2.3 \\
\hline & N127 & 3890 & 3160 & 3987 & 3679 & 452 & 184.6 & 174.1 & 173.1 & 177.3 & 6.3 \\
\hline & N129 & 327 & 307 & 312 & 315 & 11 & 49.8 & 50.5 & 45.2 & 48.5 & 2.9 \\
\hline & N130 & 157 & 181 & 161 & 166 & 13 & 59.7 & 54.9 & 59.8 & 58.1 & 2.8 \\
\hline & N131 & & & & & & & & & & \\
\hline & N132 & 330 & 330 & 340 & 333 & 6 & 60.0 & 60.0 & 60.0 & 60.0 & 0.0 \\
\hline & N133 & & & & & & & & & & \\
\hline & N134 & 277 & 223 & 220 & 240 & 32 & 48.0 & 68.0 & 37.0 & 51.0 & 15.7 \\
\hline & N140 & & & & & & 52.7 & 52.5 & 49.6 & 51.6 & 1.7 \\
\hline & N141 & & & & & & & & & & \\
\hline & N142 & 379 & 366 & 377 & 374 & 7 & 51.5 & 49.2 & 51.5 & 50.7 & 1.4 \\
\hline & N144 & & & & & & & & & & \\
\hline & N145 & 303 & 291 & 368 & 321 & 41 & 43.5 & 48.0 & 48.4 & 46.6 & 2.721 \\
\hline & N146 & & & & & & & & & & \\
\hline & N147 & & & & & & & & & & \\
\hline & N148 & 600 & 512 & 490 & 534 & 58 & 162.9 & 165.3 & 196.8 & 175.0 & 18.9 \\
\hline & N149 & 406 & 1704 & 266 & 792 & 793 & 39.2 & 51.5 & 54.8 & 48.5 & 8.2 \\
\hline & N150 & & & & & & & & & & \\
\hline & N151 & & & & & & & & & & \\
\hline & N152 & 401 & 362 & 421 & 395 & 30 & 233.9 & 206.7 & 200.1 & 213.6 & 17.9 \\
\hline & N153 & 441 & 366 & 510 & 439 & 72 & 55.0 & 50.0 & 50.0 & 51.7 & 2.9 \\
\hline & N154 & 212 & 186 & 183 & 194 & 16 & $<100$ & $<100$ & $<100$ & $<100$ & \\
\hline & N155 & 309 & 350 & 340 & 333 & 21 & 52.0 & 53.0 & 52.0 & 52.3 & 0.6 \\
\hline & N156 & 330 & 340 & 320 & 330 & 10 & 30.0 & 30.0 & 38.0 & 32.7 & 4.6 \\
\hline & N157 & $<660$ & $<660$ & $<660$ & $<660$ & & $<670$ & $<670$ & $<670$ & $<670$ & \\
\hline & N158 & 249 & 294 & 276 & 273 & 23 & 42.8 & 38.0 & 43.0 & 41.2 & 2.8 \\
\hline \multirow{5}{*}{ 蒫 } & & \multicolumn{3}{|c|}{ Consensus Mean } & \multicolumn{2}{|l|}{325} & \multicolumn{2}{|c|}{ Consensus Mean } & & 58.2 & \\
\hline & & \multicolumn{3}{|c|}{ Consensus Standard Deviation } & \multicolumn{2}{|l|}{114} & \multicolumn{3}{|c|}{ Consensus Standard Deviation } & 24.4 & \\
\hline & & \multicolumn{3}{|c|}{ Maximum } & \multicolumn{2}{|l|}{3679} & \multicolumn{3}{|c|}{ Maximum } & 213.6 & \\
\hline & & Minimum & & & 0.333 & & Minimum & & & 0.050 & \\
\hline & & $\mathrm{N}$ & & & 33 & & $\mathrm{~N}$ & & & 32 & \\
\hline
\end{tabular}


Table 5. Data summary table for cadmium in calcium and ginkgo supplements. Data highlighted in red have been flagged as potential outliers (e.g., difference from reference value, Grubb and/or Cochran) by the NIST software package.

\begin{tabular}{|c|c|c|c|c|c|c|c|c|c|c|c|}
\hline & \multirow[b]{3}{*}{ Lab } & \\
\hline & & \multicolumn{10}{|c|}{\begin{tabular}{|c|c|c|c|c|}
\multicolumn{2}{c}{ Cadmium } \\
SRM 3532 Calcium Dietary Supplement $(\mathrm{ng} / \mathrm{g})$ & SRM 3248 Ginkgo-Containing Tablets $(\mathrm{ng} / \mathrm{g})$
\end{tabular}} \\
\hline & & SRI & B & Dietary & Avg & \multirow{2}{*}{ SD } & A & 3248 Ginl & C-Contaii & Avg & SD \\
\hline \multirow{50}{*}{ 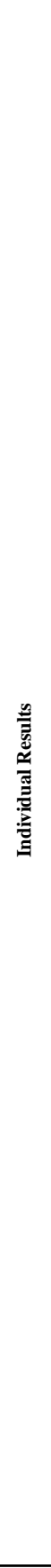 } & NIST & & & & 94.8 & & & & & & 0.18 \\
\hline & N102 & 66.0 & 62.0 & 62.0 & 63.3 & 2.3 & $<3.00$ & $<3.00$ & $<3.00$ & $<3.00$ & \\
\hline & N103 & 77.0 & 79.0 & 73.0 & 76.3 & 3.1 & 3.00 & 4.00 & 2.00 & 3.00 & 1.00 \\
\hline & N104 & 100.0 & 102.0 & 100.0 & 100.7 & 1.2 & 1.60 & 1.55 & 1.32 & 1.49 & 0.15 \\
\hline & N105 & 87.0 & 82.0 & 83.0 & 84.0 & 2.6 & $<50.00$ & 207.00 & $<50.00$ & 207.00 & \\
\hline & N106 & 96.0 & 97.0 & 93.0 & 95.3 & 2.1 & $<10.00$ & $<10.00$ & $<10.00$ & $<10.00$ & \\
\hline & N107 & 12.9 & 11.7 & 12.2 & 12.2 & 0.6 & 7.43 & 6.32 & 6.64 & 6.80 & 0.57 \\
\hline & N108 & 89.00 & 89.00 & 91.00 & 89.67 & 1.15 & 71.00 & 53.00 & 1.96 & 41.99 & 35.8 \\
\hline & N110 & 90.7 & 91.6 & 93.2 & 91.8 & 1.3 & 1.20 & 1.90 & 1.70 & 1.60 & 0.36 \\
\hline & N111 & 111.0 & 110.0 & 111.0 & 110.7 & 0.6 & 2.00 & 2.00 & 2.00 & 2.00 & 0.00 \\
\hline & N112 & 83.0 & 84.0 & 80.0 & 82.3 & 2.1 & $<10.00$ & $<10.00$ & $<10.00$ & $<10.00$ & \\
\hline & N113 & 93.9 & 89.4 & 81.1 & 88.1 & 6.5 & $<24.90$ & $<24.90$ & $<24.90$ & $<24.90$ & \\
\hline & N114 & 85.0 & 91.0 & 112.0 & 96.0 & 14.2 & $<1.000$ & 2.00 & 2.00 & 2.00 & 0.00 \\
\hline & N115 & & & & & & & & & & \\
\hline & N116 & & & & & & & & & & \\
\hline & N118 & 58.9 & 55.9 & 57.8 & 57.5 & 1.5 & 1.31 & 1.75 & 1.54 & 1.53 & 0.22 \\
\hline & N119 & 95.7 & 98.0 & 96.2 & 96.6 & 1.2 & & & & & \\
\hline & N120 & & & & & & & & & & \\
\hline & N121 & 0.10 & 0.10 & 0.10 & 0.10 & 0.00 & $<0.010$ & $<0.010$ & $<0.010$ & $<0.010$ & \\
\hline & N122 & 77.0 & 79.0 & 73.0 & 76.3 & 3.1 & 3.00 & 4.00 & 2.00 & 3.00 & 1.00 \\
\hline & $\mathrm{N} 123$ & 105.0 & 98.1 & 94.1 & 99.1 & 5.5 & & & & & \\
\hline & N124 & 95.7 & 95.2 & 102.7 & 97.8 & 4.2 & 2.15 & 1.92 & 1.94 & 2.00 & 0.13 \\
\hline & N125 & & & & & & & & & & \\
\hline & N126 & 118.0 & 118.0 & 123.0 & 119.7 & 2.9 & 1.10 & 1.50 & 1.20 & 1.27 & 0.21 \\
\hline & N127 & 92.4 & 92.0 & 85.1 & 89.8 & 4.1 & & & & & \\
\hline & N129 & 85.8 & 103.1 & 93.5 & 94.1 & 8.7 & 2.30 & 3.18 & 0.96 & 2.14 & 1.12 \\
\hline & N130 & 90.5 & 89.0 & 84.9 & 88.1 & 2.9 & 1.75 & 2.23 & 1.90 & 1.96 & 0.25 \\
\hline & N131 & & & & & & & & & & \\
\hline & $\mathrm{N} 132$ & 83.0 & 82.0 & 85.0 & 83.3 & 1.5 & 2.00 & 1.00 & 1.00 & 1.33 & 0.58 \\
\hline & N133 & & & & & & & & & & \\
\hline & N134 & 68.0 & 70.0 & 68.0 & 68.7 & 1.2 & $<10.00$ & $<10.00$ & $<10.00$ & $<10.00$ & \\
\hline & N135 & 75.5 & 75.0 & 76.2 & 75.6 & 0.6 & & & & & \\
\hline & N140 & 100.4 & 100.0 & 102.6 & 101.0 & 1.4 & & & & & \\
\hline & N141 & & & & & & & & & & \\
\hline & $\mathrm{N} 142$ & 80.7 & 94.3 & 98.2 & 91.1 & 9.2 & & & & & \\
\hline & N144 & & & & & & & & & & \\
\hline & N145 & 79.3 & 83.9 & 79.6 & 80.9 & 2.6 & & & & & \\
\hline & N146 & & & & & & & & & & \\
\hline & N147 & & & & & & & & & & \\
\hline & N148 & 54.1 & 54.2 & 49.8 & 52.7 & 2.5 & 15.70 & 17.00 & 17.20 & 16.63 & 0.81 \\
\hline & N149 & 88.8 & 82.2 & 86.3 & 85.8 & 3.3 & 2.36 & 2.14 & 1.77 & 2.09 & 0.30 \\
\hline & N150 & & & & & & & & & & \\
\hline & N151 & & & & & & & & & & \\
\hline & N152 & 87.4 & 90.0 & 89.9 & 89.1 & 1.5 & $<20.00$ & $<20.00$ & $<20.00$ & $<20.00$ & \\
\hline & N153 & 110.0 & 113.0 & 102.0 & 108.3 & 5.7 & & & & & \\
\hline & N154 & 70.0 & 71.0 & 68.0 & 69.7 & 1.5 & $<20.00$ & $<20.00$ & $<20.00$ & $<20.00$ & \\
\hline & N155 & 85.7 & 87.1 & 90.7 & 87.8 & 2.6 & $<0.50$ & $<0.50$ & $<0.50$ & $<0.50$ & \\
\hline & N156 & 74.0 & 72.0 & 76.0 & 74.0 & 2.0 & $<8.00$ & $<8.00$ & $<8.00$ & $<8.00$ & \\
\hline & N157 & $<660$ & $<660$ & $<660$ & $<660$ & & $<670$ & $<670$ & $<670$ & $<670$ & \\
\hline & N158 & 77.9 & 78.8 & 84.2 & 80.3 & 3.4 & 1.18 & 0.96 & 0.46 & 0.87 & 0.37 \\
\hline & & Consensu & & & 85.3 & & Consensu & Mean & & 2.59 & \\
\hline$\Omega$ & & Consensu & andard I & ation & 15.8 & & Consensu & Standard D & jiation & 1.69 & \\
\hline$\overline{5}$ & & Maximum & & & 119.7 & & Maximum & & & 207.00 & \\
\hline & & Minimum & & & 0.100 & & Minimum & & & 0.87 & \\
\hline & & $\mathrm{N}$ & & & 36 & & $\mathrm{~N}$ & & & 17 & \\
\hline
\end{tabular}


Table 6. Data summary table for lead in calcium and ginkgo supplements. Data highlighted in red have been flagged as potential outliers (e.g., difference from reference value, Grubb and/or Cochran) by the NIST software package.

\begin{tabular}{|c|c|c|c|c|c|c|c|c|c|c|c|}
\hline & \multirow[b]{3}{*}{ Lab } & \multicolumn{10}{|c|}{ Lead } \\
\hline & & \multicolumn{5}{|c|}{ SRM 3532 Calcium Dietary Supplement (ng/g) } & \multicolumn{5}{|c|}{ SRM 3248 Ginkgo-Containing Tablets (ng/g) } \\
\hline & & A & B & $\mathrm{C}$ & Avg & SD & $\mathbf{A}$ & B & $\mathrm{C}$ & Avg & SD \\
\hline \multirow{50}{*}{ 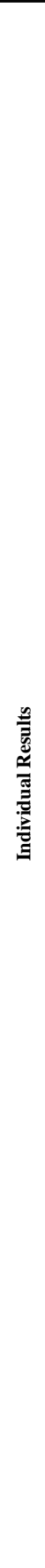 } & NIST & & & & 218 & 31 & & & & 738 & 8 \\
\hline & N102 & 214 & 209 & 201 & 208 & 7 & 715 & 696 & 706 & 706 & 10 \\
\hline & N103 & 241 & 246 & 265 & 251 & 13 & 632 & 641 & 676 & 650 & 23 \\
\hline & N104 & 270 & 312 & 227 & 270 & 43 & 692 & 692 & 702 & 695 & 6 \\
\hline & N105 & 182 & 146 & 163 & 164 & 18 & 748 & 680 & 662 & 697 & 45 \\
\hline & N106 & 270 & 240 & 220 & 243 & 25 & 700 & 720 & 710 & 710 & 10 \\
\hline & N107 & 247 & 223 & 184 & 218 & 32 & 655 & 629 & 642 & 642 & 13 \\
\hline & N108 & 214 & 229 & 271 & 238 & 30 & 727 & 689 & 727 & 714 & 22 \\
\hline & N110 & 266 & 522 & 268 & 352 & 148 & 883 & 934 & 954 & 923 & 37 \\
\hline & N111 & 252 & 261 & 280 & 264 & 14 & 850 & 844 & 852 & 849 & 4 \\
\hline & $\mathrm{N} 112$ & 255 & 191 & 216 & 221 & 32 & 671 & 675 & 669 & 672 & 3 \\
\hline & N113 & 191 & 188 & 208 & 196 & 11 & 717 & 717 & 737 & 724 & 12 \\
\hline & N114 & 177 & 259 & 263 & 233 & 49 & 824 & 768 & 770 & 787 & 32 \\
\hline & N115 & & & & & & & & & & \\
\hline & N116 & & & & & & & & & & \\
\hline & N118 & 103 & 106 & 92 & 100 & 8 & 822 & 803 & 821 & 815 & 11 \\
\hline & N119 & 229 & 304 & 272 & 268 & 38 & 689 & 704 & 701 & 698 & 8 \\
\hline & $\mathrm{N} 120$ & & & & & & & & & & \\
\hline & N121 & 0.260 & 0.290 & 0.290 & 0.280 & 0.017 & 0.780 & 0.830 & 0.770 & 0.793 & 0.032 \\
\hline & $\mathrm{N} 122$ & 241 & 246 & 265 & 251 & 13 & 632 & 641 & 675 & 649 & 23 \\
\hline & $\mathrm{N} 123$ & 186 & 206 & 195 & 196 & 10 & 804 & 780 & 726 & 770 & 40 \\
\hline & N124 & 198 & 198 & 302 & 233 & 60 & 688 & 647 & 667 & 668 & 20 \\
\hline & N125 & & & & & & & & & & \\
\hline & N126 & 98 & 186 & 106 & 130 & 49 & 709 & 708 & 689 & 702 & 11 \\
\hline & N127 & 282 & 232 & 285 & 266 & 29 & 718 & 689 & 674 & 694 & 23 \\
\hline & N129 & 185 & 171 & 300 & 219 & 71 & 712 & 728 & 685 & 708 & 22 \\
\hline & $\mathrm{N} 130$ & 239 & 324 & 224 & 262 & 54 & 712 & 732 & 715 & 720 & 11 \\
\hline & N131 & & & & & & & & & & \\
\hline & $\mathrm{N} 132$ & 180 & 190 & 200 & 190 & 10 & 660 & 680 & 680 & 673 & 12 \\
\hline & N133 & & & & & & & & & & \\
\hline & $\mathrm{N} 134$ & 211 & 211 & 213 & 212 & 1 & 711 & 732 & 736 & 726 & 13 \\
\hline & N135 & 167 & 170 & 168 & 168 & 2 & 501 & 492 & 500 & 498 & 5 \\
\hline & N140 & 235 & 247 & 236 & 239 & 7 & 780 & 780 & 790 & 783 & 6 \\
\hline & N141 & & & & & & & & & & \\
\hline & N142 & 255 & 244 & 243 & 247 & 7 & 763 & 770 & 773 & 769 & 5 \\
\hline & N144 & & & & & & & & & & \\
\hline & N145 & 229 & 190 & 217 & 212 & 20 & 690 & 774 & 720 & 728 & 43 \\
\hline & N146 & & & & & & & & & & \\
\hline & N147 & & & & & & & & & & \\
\hline & N148 & 241 & 206 & 244 & 230 & 21 & 724 & 829 & 934 & 829 & 105 \\
\hline & N149 & 209 & 221 & 85 & 172 & 75 & 685 & 707 & 711 & 701 & 14 \\
\hline & N150 & & & & & & & & & & \\
\hline & N151 & & & & & & & & & & \\
\hline & N152 & 343 & 251 & 210 & 268.0 & 68.1 & 792 & 773 & 786 & 783.5 & 9.4 \\
\hline & N153 & 207 & 291 & 215 & 238 & 46 & 739 & 720 & 673 & 711 & 34 \\
\hline & N154 & 166 & 169 & 150 & 162 & 10 & 563 & 558 & 559 & 560 & 3 \\
\hline & N155 & 230 & 240 & 358 & 276 & 71 & 761 & 772 & 772 & 768 & 6 \\
\hline & N156 & 230 & 230 & 250 & 237 & 12 & 690 & 700 & 690 & 693 & 6 \\
\hline & N157 & 800 & 730 & 730 & 753 & 40 & $<670$ & $<670$ & $<670$ & $<670$ & \\
\hline & N158 & 165 & 171 & 168 & 168 & 3 & 584 & 609 & 587 & 593 & 14 \\
\hline \multirow{5}{*}{ 象 } & & \multicolumn{3}{|c|}{ Consensus Mean } & \multicolumn{2}{|l|}{223} & \multicolumn{3}{|c|}{ Consensus Mean } & 712 & \\
\hline & & \multicolumn{3}{|c|}{ Consensus Standard Deviation } & \multicolumn{2}{|l|}{51} & \multicolumn{3}{|c|}{ Consensus Standard Deviation } & 71 & \\
\hline & & \multicolumn{3}{|c|}{ Maximum } & \multicolumn{2}{|l|}{753} & Maximum & & & 923 & \\
\hline & & Minimum & & & 0.280 & & Minimum & & & 0.793 & \\
\hline & & $\mathrm{N}$ & & & 37 & & $\mathrm{~N}$ & & & 36 & \\
\hline
\end{tabular}




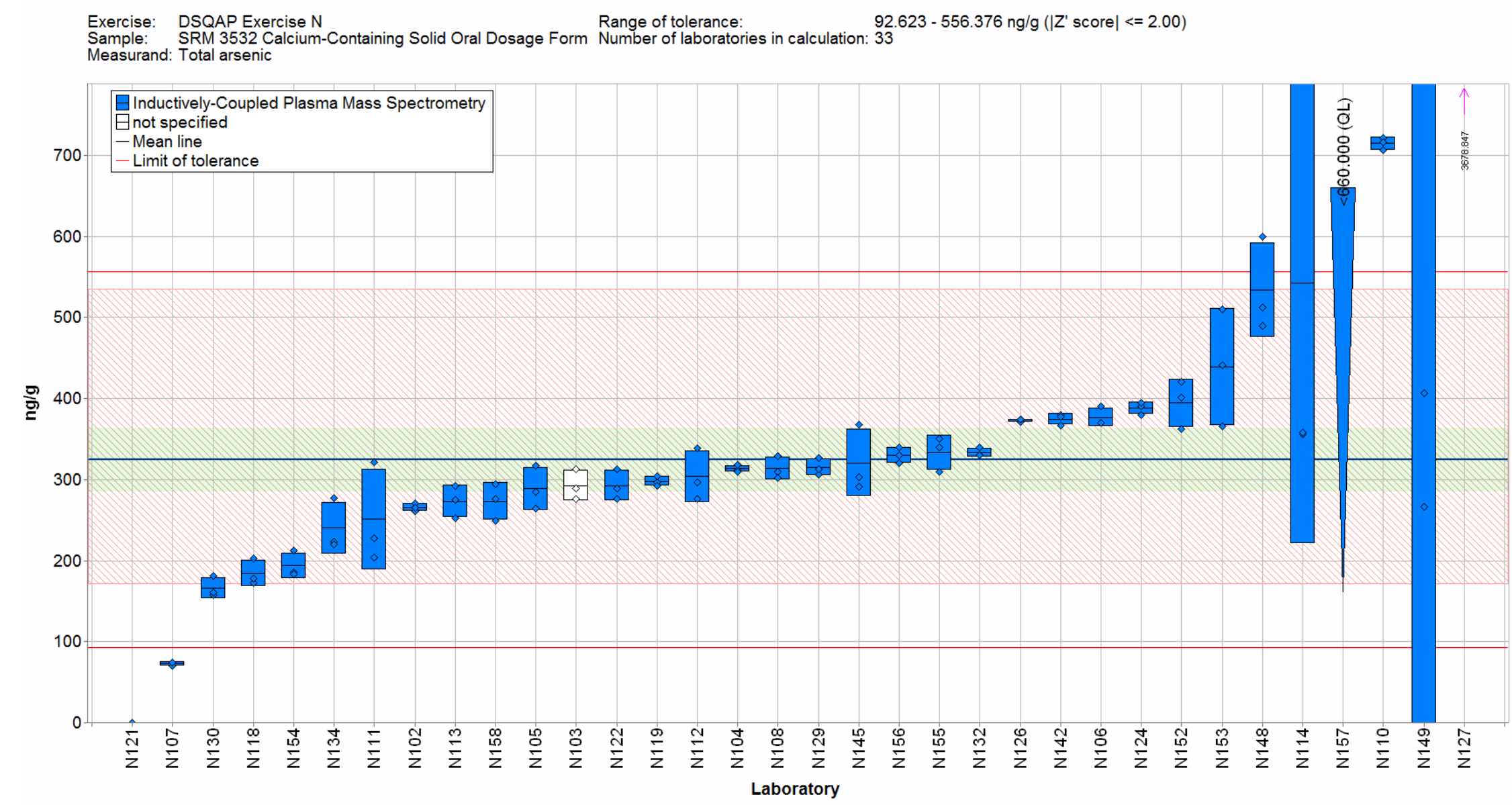

Figure 4. Arsenic in SRM 3532 Calcium-Containing Solid Oral Dosage Form (data summary view - analytical method). In this view, individual laboratory data are plotted (diamonds) with the individual laboratory standard deviation (rectangle). The color of the data point represents the analytical method employed. Laboratory data shown as a triangle indicates that a "less than" result was submitted, and the base of the triangle is displayed at the reported laboratory detection limit. The solid blue line represents the consensus mean and the green shaded region represents the $95 \%$ confidence interval for the consensus mean. The solid red lines represent the consensus range of tolerance, calculated as the values above and below the consensus mean that result in an acceptable $Z_{\text {comm }}^{\prime}$ score, $\left|Z_{\text {comm }}^{\prime}\right| \leq 2$. The red shaded region represents the NIST range of tolerance, which encompasses the NIST-determined value bounded by twice its uncertainty $\left(U_{95}\right)$ and represents the range that results in an acceptable $Z_{\text {NIST }}$ score, $\left|Z_{\text {NIST }}\right| \leq 2$. 


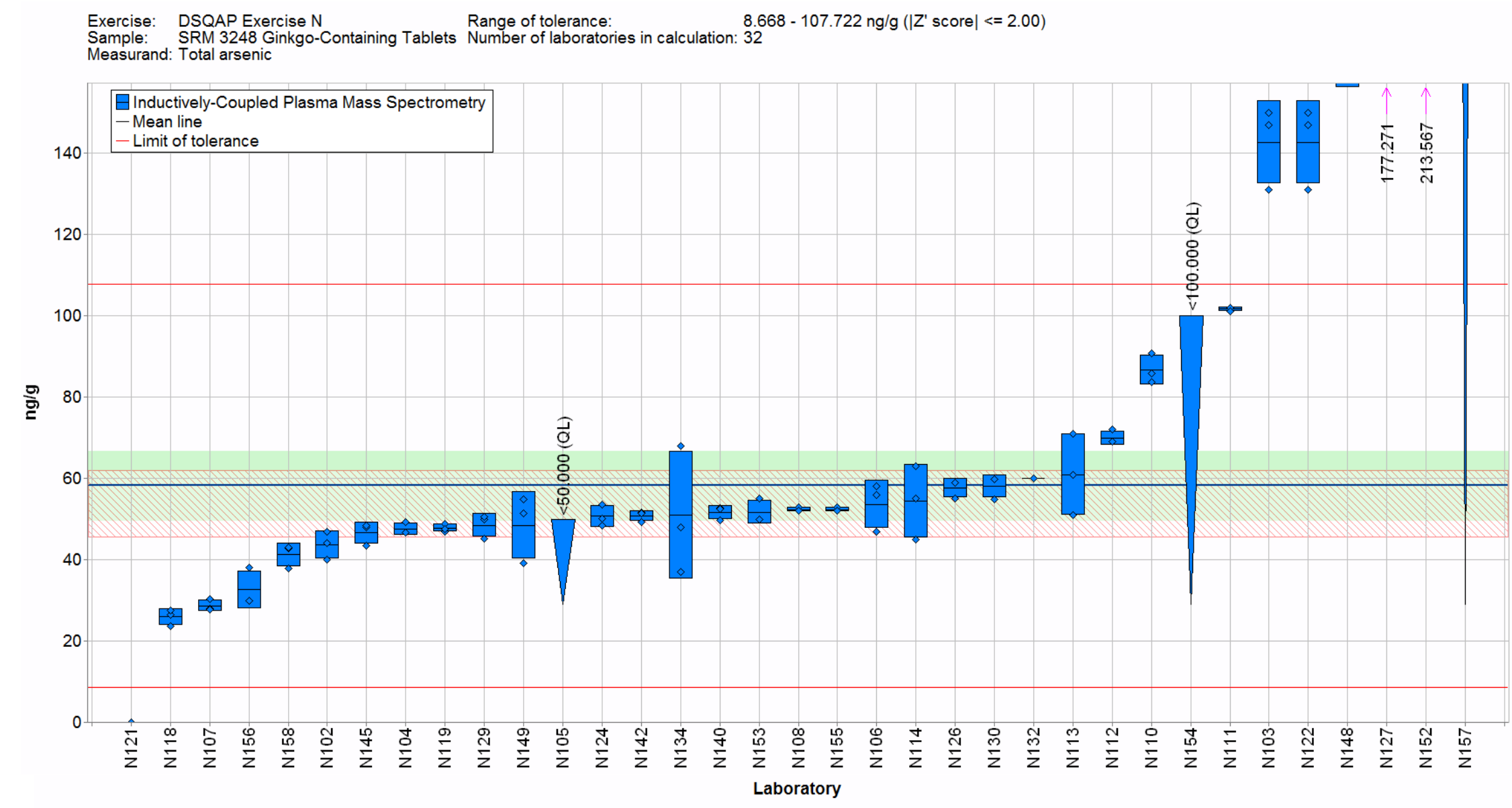

Figure 5. Arsenic in SRM 3248 Ginkgo-Containing Tablets (data summary view - analytical method). In this view, individual laboratory data are plotted (diamonds) with the individual laboratory standard deviation (rectangle). The color of the data point represents the analytical method employed. Laboratory data shown as a triangle indicates that a "less than" result was submitted, and the base of the triangle is displayed at the reported laboratory detection limit. The solid blue line represents the consensus mean and the green shaded region represents the $95 \%$ confidence interval for the consensus mean. The solid red lines represent the consensus range of tolerance, calculated as the values above and below the consensus mean that result in an acceptable $Z_{\text {comm }}^{\prime}$ score, $\left|Z_{\text {comm }}^{\prime}\right| \leq 2$. The red shaded region represents the NIST range of tolerance, which encompasses the NIST reference value bounded by twice its uncertainty $\left(U_{95}\right)$ and represents the range that results in an acceptable $Z_{\mathrm{NIST}}$ score, $\left|Z_{\mathrm{NIST}}\right| \leq 2$. 


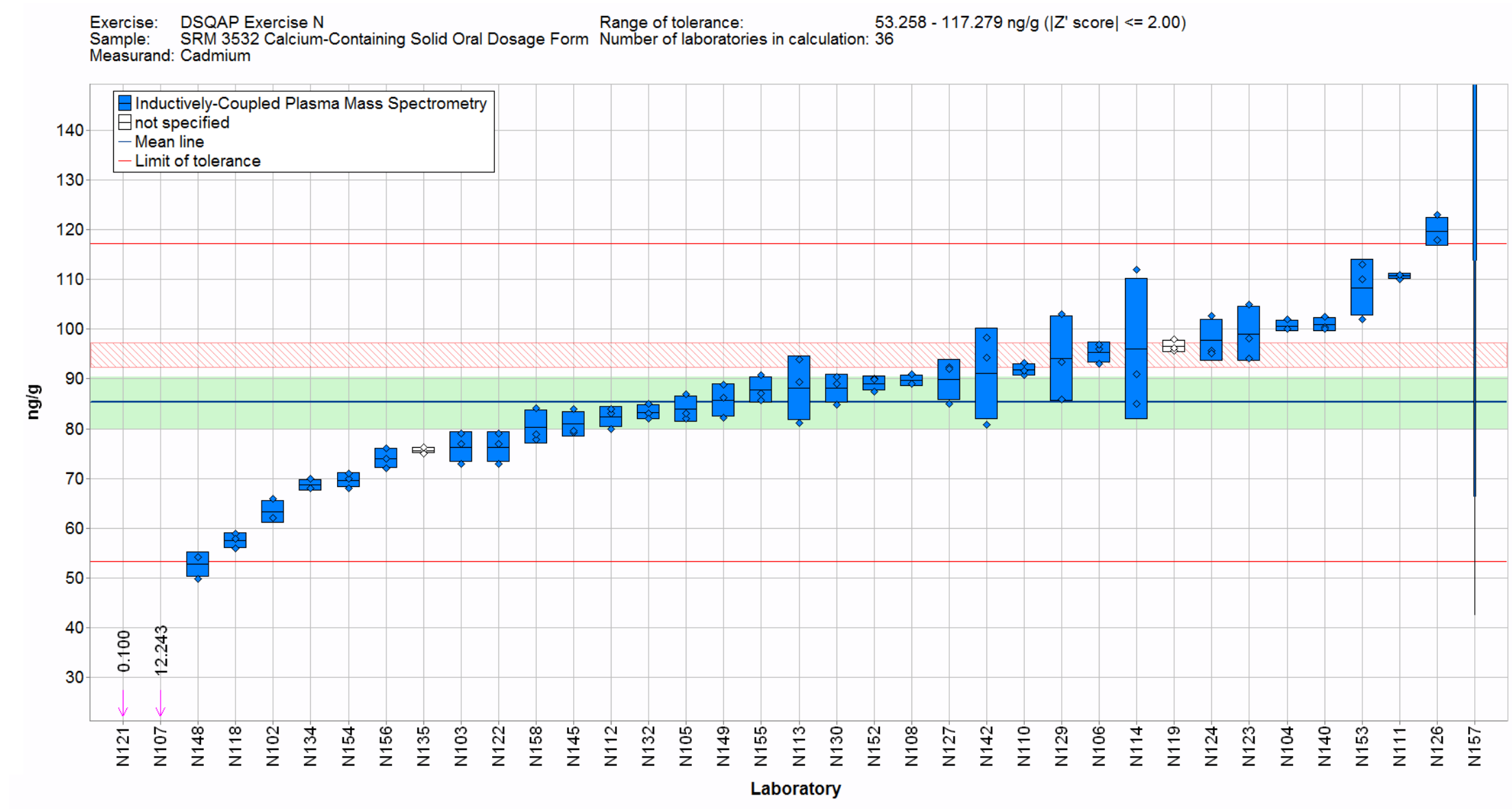

Figure 6. Cadmium in SRM 3532 Calcium-Containing Solid Oral Dosage Form (data summary view - analytical method). In this view, individual laboratory data are plotted (diamonds) with the individual laboratory standard deviation (rectangle). The color of the data point represents the analytical method employed. Laboratory data shown as a triangle indicates that a "less than" result was submitted, and the base of the triangle is displayed at the reported laboratory detection limit. The solid blue line represents the consensus mean and the green shaded region represents the $95 \%$ confidence interval for the consensus mean. The solid red lines represent the consensus range of tolerance, calculated as the values above and below the consensus mean that result in an acceptable $Z_{\text {comm }}^{\prime}$ score, $\left|Z_{\text {comm }}^{\prime}\right| \leq 2$. The red shaded region represents the NIST range of tolerance, which encompasses the NIST certified value bounded by twice its uncertainty $\left(U_{95}\right)$ and represents the range that results in an acceptable $Z_{\text {NIST }}$ score, $\left|Z_{\text {NIST }}\right| \leq 2$. 


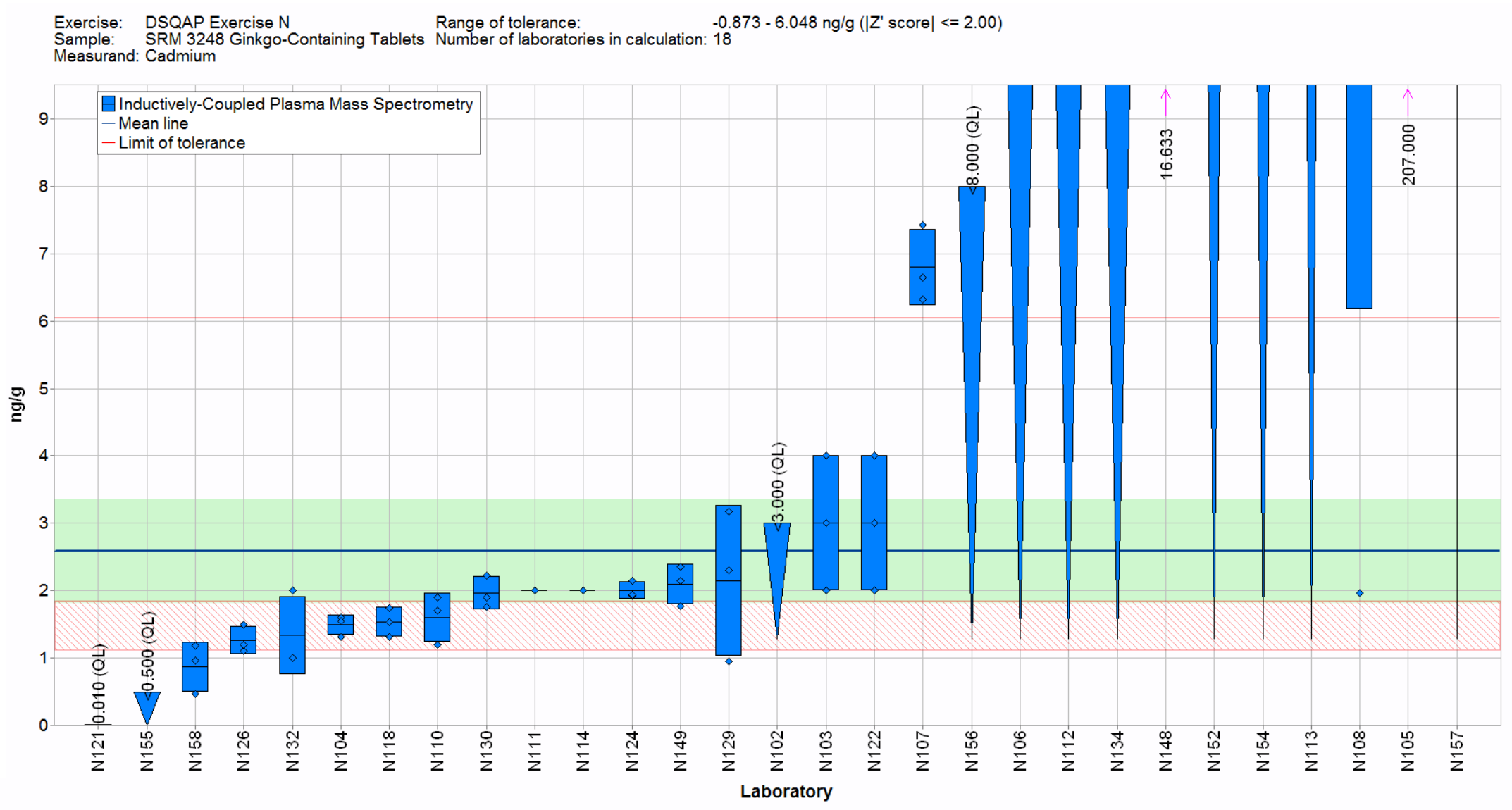

Figure 7. Cadmium in SRM 3248 Ginkgo-Containing Tablets (data summary view - analytical method). In this view, individual laboratory data are plotted (diamonds) with the individual laboratory standard deviation (rectangle). The color of the data point represents the analytical method employed. Laboratory data shown as a triangle indicates that a "less than" result was submitted, and the base of the triangle is displayed at the reported laboratory detection limit. The solid blue line represents the consensus mean and the green shaded region represents the $95 \%$ confidence interval for the consensus mean. The solid red line represents the upper consensus range of tolerance, calculated as the values above and below the consensus mean that result in an acceptable $Z_{\text {comm }}^{\prime}$ score, $\left|Z_{\text {comm }}^{\prime}\right| \leq 2$, with the lower range set at zero. The red shaded region represents the NIST range of tolerance, which encompasses the NIST reference value bounded by twice its uncertainty $\left(U_{95}\right)$ and represents the range that results in an acceptable $Z_{\text {NIST }}$ score, $\left|Z_{\text {NIST }}\right| \leq 2$. 


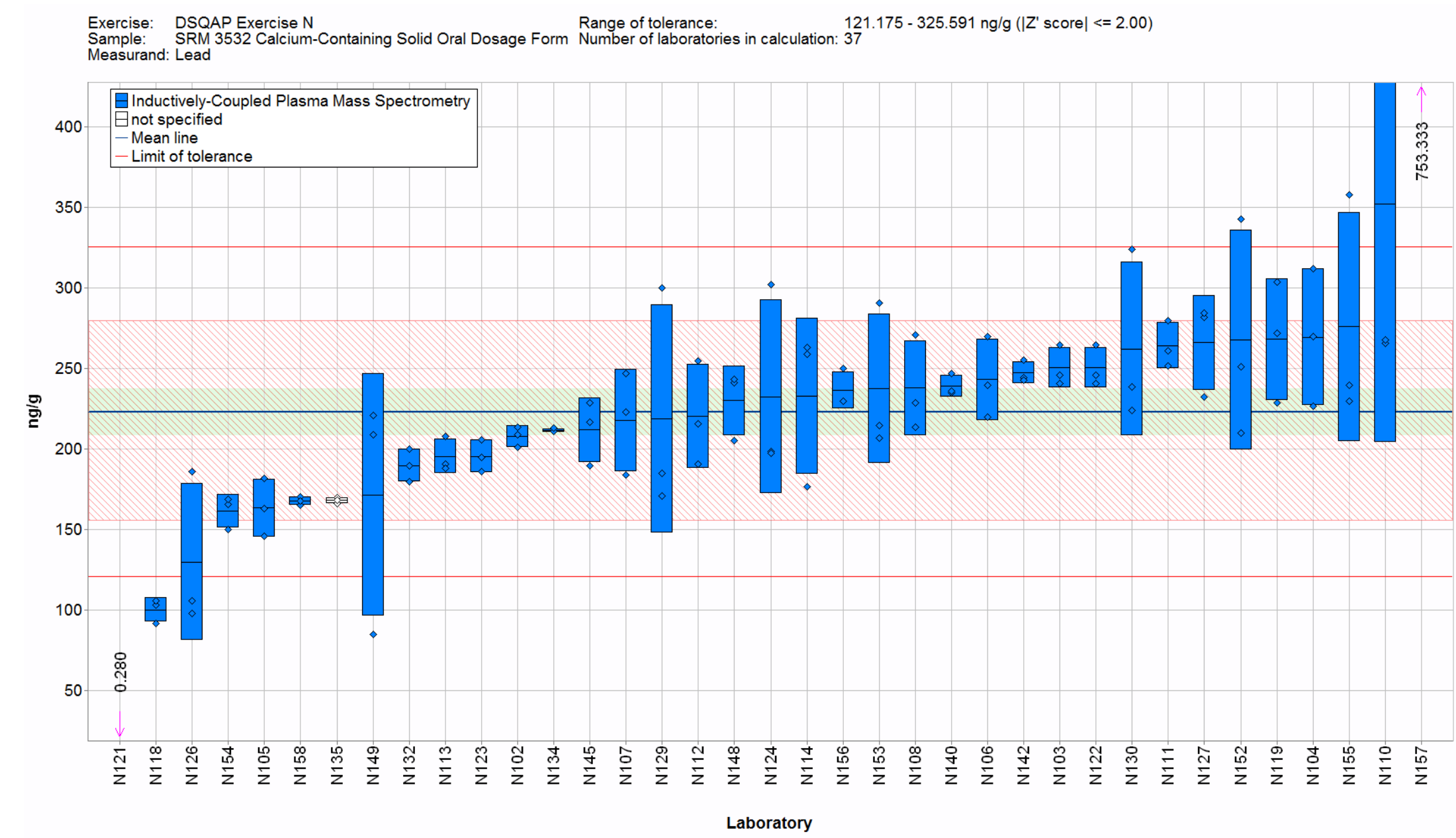

Figure 8. Lead in SRM 3532 Calcium-Containing Solid Oral Dosage Form (data summary view - analytical method). In this view, individual laboratory data are plotted (diamonds) with the individual laboratory standard deviation (rectangle). The color of the data point represents the analytical method employed. The solid blue line represents the consensus mean and the green shaded region represents the $95 \%$ confidence interval for the consensus mean. The solid red lines represent the consensus range of tolerance, calculated as the values above and below the consensus mean that result in an acceptable $Z_{\text {comm }}^{\prime}$ score, $\left|Z_{\text {comm }}^{\prime}\right| \leq 2$. The red shaded region represents the NIST range of tolerance, which encompasses the NIST reference value bounded by twice its uncertainty ( $\left.U_{95}\right)$ and represents the range that results in an acceptable $Z_{\text {NIST }}$ score, $\left|Z_{\text {NIST }}\right| \leq 2$. 


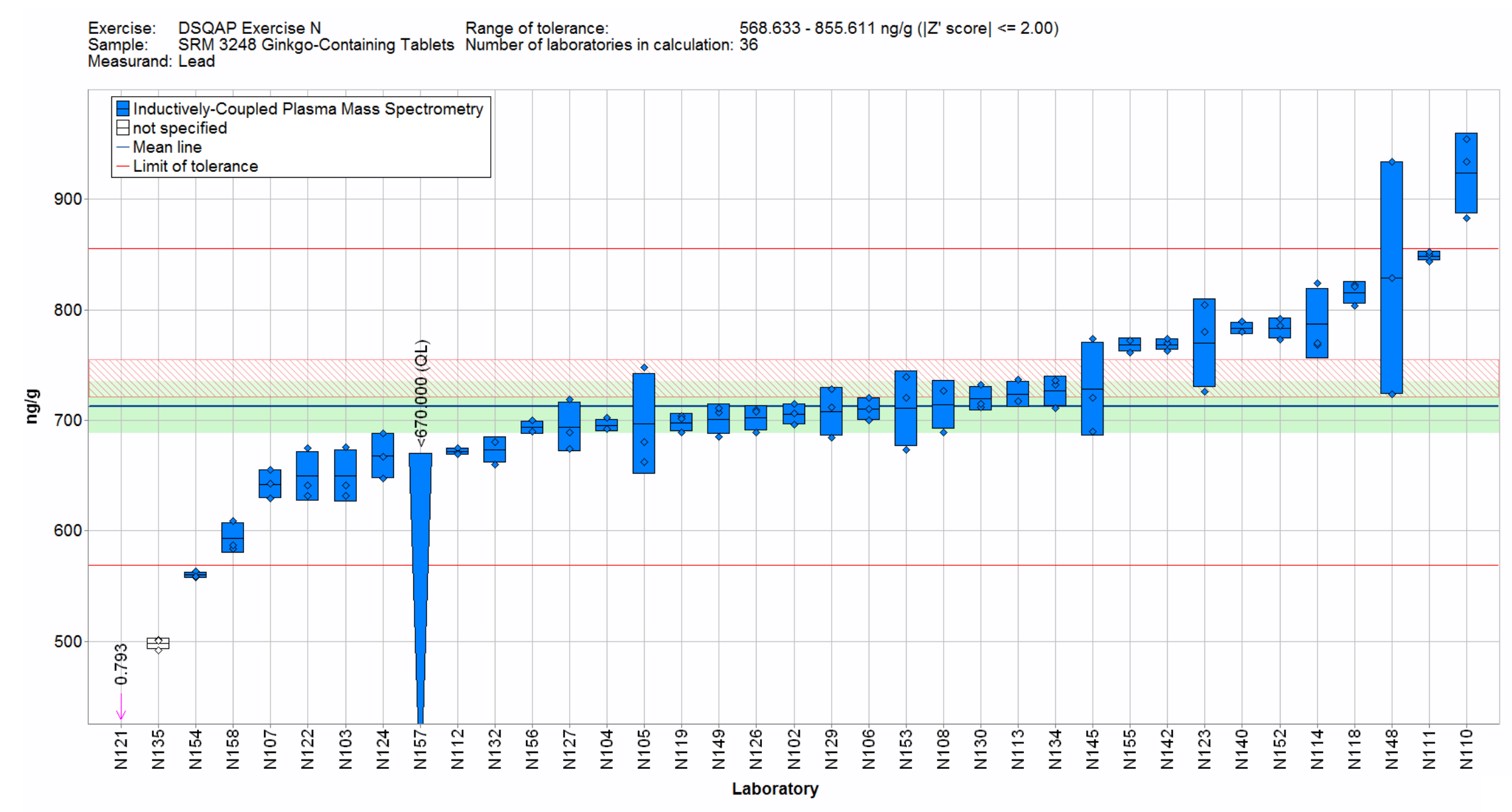

Figure 9. Lead in SRM 3248 Ginkgo-Containing Tablets (data summary view - analytical method). In this view, individual laboratory data are plotted (diamonds) with the individual laboratory standard deviation (rectangle). The color of the data point represents the analytical method employed. Laboratory data shown as a triangle indicates that a "less than" result was submitted, and the base of the triangle is displayed at the reported laboratory detection limit. The solid blue line represents the consensus mean and the green shaded region represents the $95 \%$ confidence interval for the consensus mean. The solid red lines represent the consensus range of tolerance, calculated as the values above and below the consensus mean that result in an acceptable $Z_{\text {comm }}^{\prime}$ score, $\left|Z_{\text {comm }}^{\prime}\right| \leq 2$. The red shaded region represents the NIST range of tolerance, which encompasses the NIST certified value bounded by twice its uncertainty ( $\left.U_{95}\right)$ and represents the range that results in an acceptable $Z_{\text {NIST }}$ score, $\left|Z_{\text {NIST }}\right| \leq 2$. 


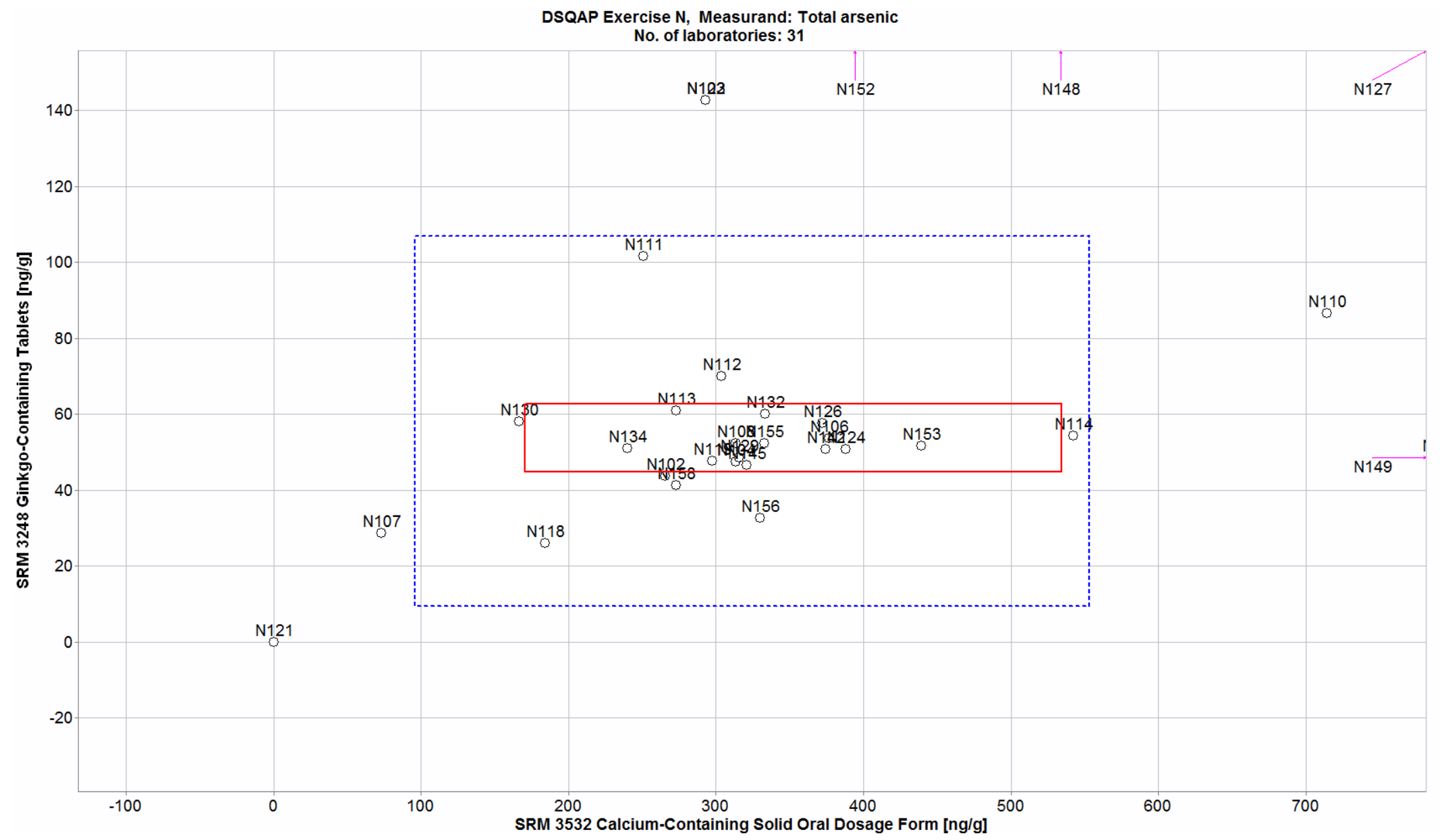

Figure 10. Laboratory means for total arsenic in SRM 3532 Calcium-Containing Solid Oral Dosage Form and SRM 3248 Ginkgo-Containing Tablets (sample/sample comparison view). In this view, the individual laboratory mean for one sample (calcium supplement) is compared to the mean for a second sample (ginkgo tablets). The solid red box represents the NIST range of tolerance for the two samples, calcium supplement (x-axis) and ginkgo tablets (y-axis), which encompasses the NIST values bounded by their uncertainties ( $\left.U_{95}\right)$ and represents the range that results in an acceptable $Z_{\text {NIST }}$ score, $\left|Z_{\text {NIST }}\right| \leq 2$. The dotted blue box represents the consensus range of tolerance for calcium supplement ( $\mathrm{x}$-axis) and ginkgo tablets (y-axis), calculated as the values above and below the consensus means that result in an acceptable $Z_{\text {comm }}^{\prime}$ score, $\left|Z_{\text {comm }}^{\prime}\right| \leq 2$. 


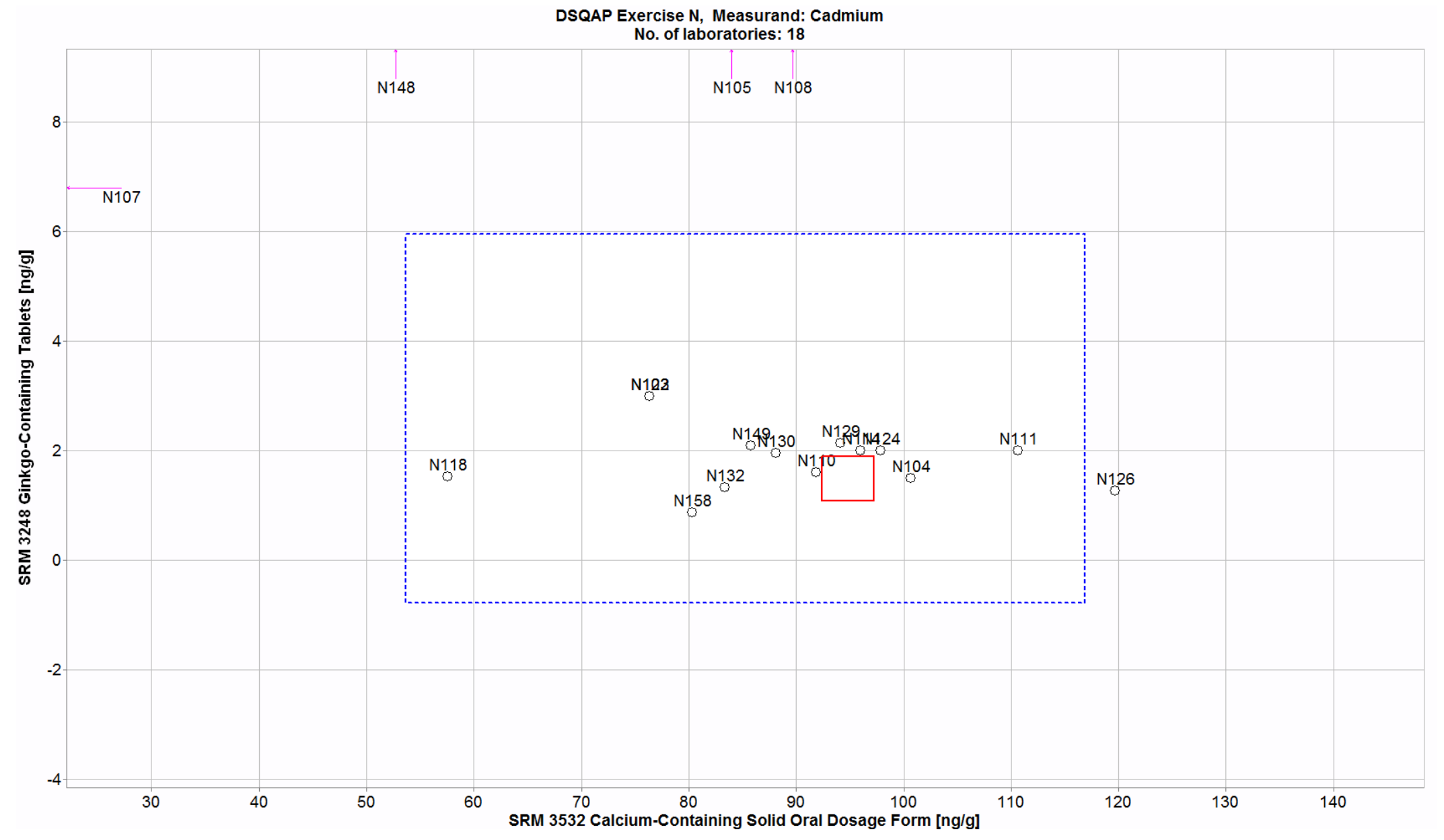

Figure 11. Laboratory means for cadmium in SRM 3532 Calcium-Containing Solid Oral Dosage Form and SRM 3248 Ginkgo-Containing Tablets (sample/sample comparison view). In this view, the individual laboratory mean for one sample (calcium supplement) is compared to the mean for a second sample (ginkgo tablets). The solid red box represents the NIST range of tolerance for the two samples, calcium supplement (x-axis) and ginkgo tablets (y-axis), which encompasses the NIST values bounded by their uncertainties ( $\left.U_{95}\right)$ and represents the range that results in an acceptable $Z_{\mathrm{NIST}}$ score, $\left|Z_{\mathrm{NIST}}\right| \leq 2$. The dotted blue box represents the consensus range of tolerance for calcium supplements ( $\mathrm{x}$-axis) and ginkgo tablets (y-axis), calculated as the values above and below the consensus means that result in an acceptable $Z_{\text {comm }}^{\prime}$ score, $\left|Z_{\text {comm }}^{\prime}\right| \leq 2$. 


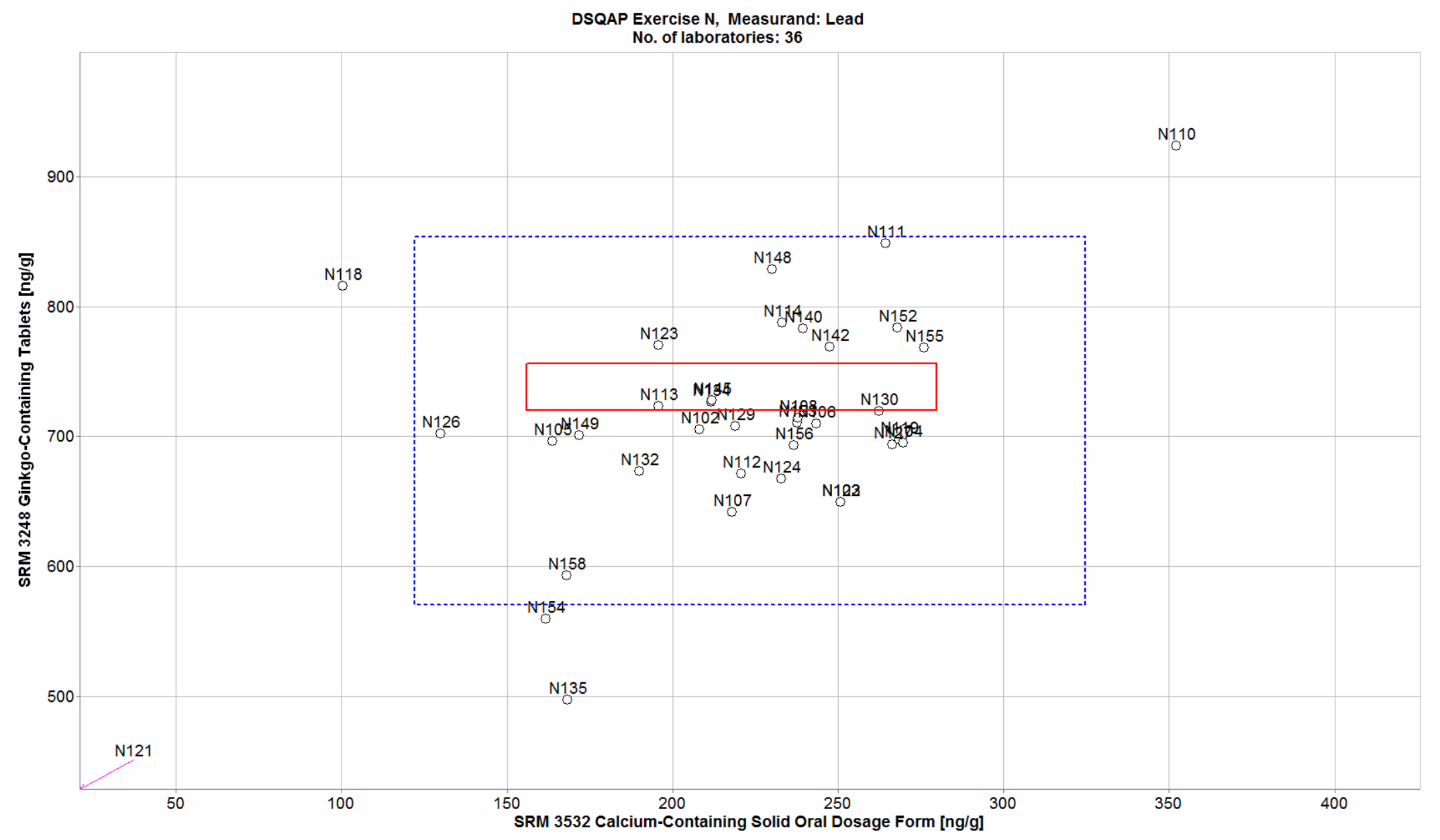

Figure 12. Laboratory means for lead in SRM 3532 Calcium-Containing Solid Oral Dosage Form and SRM 3248 Ginkgo-Containing Tablets (sample/sample comparison view). In this view, the individual laboratory mean for one sample (calcium supplement) is compared to the mean for a second sample (ginkgo tablets). The solid red box represents the NIST range of tolerance for the two samples, calcium supplement (x-axis) and ginkgo tablets (y-axis), which encompasses the NIST values bounded by their uncertainties $\left(U_{95}\right)$ and represents the range that results in an acceptable $Z_{\text {NIST }}$ score, $\left|Z_{\mathrm{NIST}}\right| \leq 2$. The dotted blue box represents the consensus range of tolerance for calcium supplement (x-axis) and ginkgo tablet (y-axis), calculated as the values above and below the consensus means that result in an acceptable $Z_{\text {comm }}^{\prime}$ score, $\left|Z_{\text {comm }}^{\prime}\right| \leq 2$. 


\section{WATER-SOLUBLE VITAMINS (FOLIC ACID) IN FOODS AND DIETARY SUPPLEMENTS}

$\underline{\text { Study Overview }}$

In this study, participants were provided with two NIST SRMs, SRM 3280 Multivitamin/Multielement Tablets, and SRM 3233 Fortified Breakfast Cereal. Participants were asked to use in-house analytical methods to determine the mass fraction of folic acid in each of the matrices and report values in $\mathrm{mg} / \mathrm{kg}$ on an as-received basis.

$\underline{\text { Sample Information }}$

Multivitamin. Participants were provided with one bottle containing 30 multivitamin/multielement tablets. Before use, participants were instructed to grind all tablets, mix the resulting powder thoroughly, and to use a sample size of at least $0.3 \mathrm{~g}$. Participants were asked to store the material at controlled room temperature, $10{ }^{\circ} \mathrm{C}$ to $30^{\circ} \mathrm{C}$, and to prepare three samples and report three values from the single bottle provided. The approximate analyte level was not reported to participants prior to the study. The certified value for folic acid in SRM 3280 was determined at NIST using isotope dilution liquid chromatography with tandem mass spectrometry detection (ID-LC-MS/MS) in combination with data from collaborating laboratories. The certified value and uncertainty for folic acid is provided in the table below, both on a dry-mass basis and on an as-received basis accounting for moisture of the material (1.37\%).

$\begin{array}{ccc}\text { Analyte } & \begin{array}{l}\text { Certified Mass Fraction in SRM } 3280(\mathrm{mg} / \mathrm{kg}) \\ \text { (dry-mass basis) }\end{array} & \begin{array}{c}\text { (as-received basis) } \\ \text { Folic Acid }\end{array} \\ 394 \pm 22 & 389 \pm 22\end{array}$

Breakfast Cereal. Participants were provided with three packets containing at least $3 \mathrm{~g}$ of ground cereal. The commercial cereal was ground, homogenized, and heat-sealed inside 4 mil polyethylene bags which were then sealed inside nitrogen-flushed aluminized plastic bags along with two packets of silica gel each. Before use, participants were instructed to thoroughly mix the contents of each packet and use a sample size of at least $1 \mathrm{~g}$. Participants were asked to store the material at controlled room temperature, $10{ }^{\circ} \mathrm{C}$ to $30^{\circ} \mathrm{C}$, and to prepare one sample and report one value from each packet provided. The approximate analyte level was not reported to participants prior to the study. The certified value for folic acid in SRM 3233 was determined at NIST using ID-LC-MS/MS in combination with data from collaborating laboratories. The certified value and uncertainty for folic acid is provided in the table below, both on a dry-mass basis and on an as-received basis accounting for moisture of the material (1.7\%).

$\begin{array}{ccc}\underline{\text { Analyte }} & \begin{array}{c}\text { Certified Mass Fraction in SRM } 3233(\mathrm{mg} / \mathrm{kg}) \\ \text { (dry-mass basis) }\end{array} & \begin{array}{c}\text { (as-received basis) } \\ \text { Folic Acid }\end{array} \\ 15.1 \pm 1.2 & 14.8 \pm 1.2\end{array}$

$\underline{\text { Study Results }}$

- Thirty-three laboratories enrolled in this exercise and received samples. Sixteen laboratories reported results for folic acid in the cereal (48\% participation) and 25 laboratories reported results for folic acid in multivitamin (76\% participation). 
- The consensus mean for folic acid in the multivitamin was within the target range and the between-laboratory variability was very good (9\% RSD).

- The consensus mean for folic acid in the breakfast cereal was below the target range, but the consensus range overlapped the target range. The between-laboratory variability was high (33\% RSD).

- Most laboratories reported using LC with absorbance detection as the analytical method for analysis (65\% for breakfast cereal and $70 \%$ for the multivitamin). Laboratories also reported using a microbiological assay for breakfast cereal (6\%), LC-MS/MS (6 \% for cereal and $4 \%$ for the multivitamin), and a USP assay for the multivitamin (4\%). Several laboratories did not report an instrumental approach $(24 \%$ for cereal or $15 \%$ for multivitamin).

\section{Technical Recommendations}

The following recommendations are based on results obtained from the participants in this study.

- Results for the multivitamin tablet were very good. No methods were presented as significantly better or worse than any other. No systematic biases were noted.

- Some laboratories had high sample-to-sample variability, which may be related to tabletto-tablet variability if the recommended sample preparation procedure was not followed. The recommended sample preparation procedure included grinding all 30 provided tablets together and sampling from the resulting powder to reduce sample-to-sample variability. Additional variability may have resulted from exposure of the ground powder to light and/or air during sample preparation, if samples were not prepared in rapid succession.

- The low bias of the consensus results may indicate incomplete extraction of the high concentration of folic acid from the matrix. A subsequent re-extraction of the sample can indicate if measurable amounts of the analyte remain in the matrix following extraction.

- Results for the breakfast cereal were highly variable. However, no methods were presented as significantly better or worse than any other and no systematic biases were noted.

- The mass fraction of the folic acid in the breakfast cereal was significantly lower than the multivitamin sample. A linear calibration curve which surrounds the expected sample concentration values, including both the lowest and highest expected concentration values of the sample solutions, should be used for calculation. Extrapolation of results beyond calibration curves may result in inaccuracies in sample results.

- Neat folic acid standards are known to contain high levels of water (up to $8 \%$ or more), which would not be detectable by LC-absorbance or LC-MS purity approaches. The purity of reference standards should always be thoroughly characterized in-house to reduce potential bias.

- Some laboratories reported high values for one sample or the other (but not both), which may indicate chromatographic coelutions with matrix components. Because each matrix is different, the chromatographic method should be evaluated to confirm any potential biases resulting from interfering compounds. 
Table 7. Individualized data summary table (NIST) for folic acid in multivitamin and breakfast cereal.

\section{National Institute of Standards \& Technology}

\begin{tabular}{|c|c|c|c|c|c|c|c|c|c|c|c|}
\hline \multirow[b]{2}{*}{ Analyte } & \multirow{2}{*}{$\frac{\text { Lab Code: }}{\text { Sample }}$} & \multirow{2}{*}{$\begin{array}{l}\text { NIST } \\
\text { Units }\end{array}$} & \multicolumn{4}{|c|}{$\begin{array}{c}\text { Exercise N - May } 2017 \text { - Folic Acid } \\
\text { 1. Your Results }\end{array}$} & \multicolumn{3}{|c|}{ 2. Community Results } & \multicolumn{2}{|c|}{ 3. Target } \\
\hline & & & $\mathrm{x}_{\mathrm{i}}$ & $\mathrm{s}_{\mathrm{i}}$ & $Z_{\text {comm }}^{\prime}$ & $\mathrm{Z}_{\mathrm{NIST}}$ & $\mathrm{N}$ & $\mathrm{x}^{*}$ & $\mathrm{~s}^{*}$ & $\mathrm{x}_{\mathrm{NIST}}$ & $U_{95}$ \\
\hline Folic Acid & Cereal & $\mathrm{mg} / \mathrm{kg}$ & 14.8 & 1.2 & & 0.00 & 16 & 13 & 5 & 14.8 & 1.2 \\
\hline Folic Acid & Multivitamin & $\mathrm{mg} / \mathrm{kg}$ & 389 & 22 & & 0.00 & 25 & 370 & 33 & 389 & 22 \\
\hline
\end{tabular}

\author{
$\mathrm{x}_{\mathrm{i}}$ Mean of reported values \\ $\mathrm{s}_{\mathrm{i}}$ Standard deviation of reported values \\ $Z_{\text {comm }}^{\prime} Z^{\prime}$-score with respect to community \\ consensus \\ $\mathrm{Z}_{\text {NIST }}$ Z-score with respect to NIST value
}

$\mathrm{N}$ Number of quantitative values reported

$\mathrm{x}^{*}$ Robust mean of reported values

$\mathrm{s}^{*}$ Robust standard deviation
$\mathrm{x}_{\mathrm{NIST}}$ NIST-assessed value $U_{95} \pm 95 \%$ confidence interval about the assessed value or standard deviation $\left(\mathrm{s}_{\mathrm{NIST}}\right)$ 
Table 8. Data summary table for folic acid in multivitamin and in breakfast cereal. Data highlighted in red have been flagged as potential outliers (e.g., difference from reference value, Grubb and/or Cochran) by the NIST software package.

\begin{tabular}{|c|c|c|c|c|c|c|c|c|c|c|c|}
\hline & \multirow[b]{3}{*}{ Lab } & \multicolumn{10}{|c|}{ Folic Acid } \\
\hline & & \multicolumn{5}{|c|}{ SRM 3233 Fortified Breakfast Cereal $(\mathrm{mg} / \mathrm{kg})$} & \multicolumn{5}{|c|}{ SRM 3280 Multivitamin/Multielement Tablets $(\mathrm{mg} / \mathrm{kg}$ ) } \\
\hline & & $\mathbf{A}$ & B & C & Avg & SD & $\mathbf{A}$ & B & C & Avg & SD \\
\hline \multirow{34}{*}{ 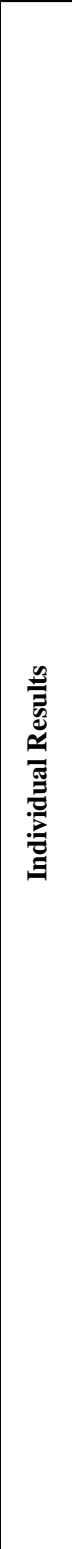 } & NIST & & & & 14.8 & 1.2 & & & & 389 & 22 \\
\hline & N101 & & & & & & 412 & 398 & 376 & 395 & 18 \\
\hline & N102 & 10.9 & 11.0 & 9.8 & 10.6 & 0.7 & 384 & 379 & 371 & 378 & 7 \\
\hline & N103 & & & & & & & & & & \\
\hline & $\mathrm{N} 105$ & & & & & & 410 & 406 & 403 & 406 & 4 \\
\hline & N107 & 86.8 & 88.1 & 85.4 & 86.8 & 1.4 & 368 & 335 & 320 & 341 & 25 \\
\hline & N108 & 10.0 & 10.0 & 9.9 & 10.0 & 0.0 & 385 & 381 & 380 & 382 & 3 \\
\hline & N110 & 19.1 & 18.4 & 19.4 & 19.0 & 0.5 & 385 & 383 & 376 & 381 & 5 \\
\hline & N111 & 39.0 & 44.0 & 38.0 & 40.3 & 3.2 & 336 & 321 & 353 & 337 & 16 \\
\hline & $\mathrm{N} 112$ & 15.4 & 15.7 & 15.7 & 15.6 & 0.2 & 392 & 382 & 359 & 378 & 17 \\
\hline & $\mathrm{N} 113$ & 0.0 & 0.0 & 0.0 & 0.0 & 0.0 & 0 & 0 & 0 & 0 & 0 \\
\hline & $\mathrm{N} 115$ & & & & & & & & & & \\
\hline & N116 & 9.2 & 8.8 & 8.6 & 8.8 & 0.3 & 355 & 372 & 383 & 370 & 14 \\
\hline & N117 & & & & & & 368 & 361 & 373 & 367 & 6 \\
\hline & N120 & & & & & & & & & & \\
\hline & N121 & & & & & & 292 & 290 & 295 & 292 & 3 \\
\hline & $\mathrm{N} 122$ & & & & & & & & & & \\
\hline & N123 & 14.5 & 15.7 & 14.4 & 14.9 & 0.7 & 335 & 333 & 342 & 337 & 5 \\
\hline & N124 & & & & & & 359 & 355 & 358 & 357 & 2 \\
\hline & N125 & & & & & & & & & & \\
\hline & N126 & 10.5 & 9.1 & 10.3 & 10.0 & 0.7 & 388 & 394 & 409 & 397 & 10 \\
\hline & N127 & 11.4 & 10.7 & 11.4 & 11.2 & 0.4 & 384 & 370 & 398 & 384 & 14 \\
\hline & N129 & 11.8 & 12.1 & 12.1 & 12.0 & 0.2 & 389 & 390 & 385 & 388 & 3 \\
\hline & N130 & & & & & & 354 & 349 & 354 & 353 & 3 \\
\hline & $\mathrm{N} 132$ & 14.5 & 14.4 & 14.4 & 14.4 & 0.1 & 453 & 439 & 449 & 447 & 7 \\
\hline & N133 & & & & & & 378 & 361 & 373 & 371 & 9 \\
\hline & N138 & & & & & & 421 & 427 & 426 & 425 & 3 \\
\hline & N141 & & & & & & & & & & \\
\hline & N143 & 15.1 & 15.1 & 15.1 & 15.1 & 0.0 & 416 & 411 & 412 & 413 & 3 \\
\hline & N148 & 7.8 & 8.3 & 8.8 & 8.3 & 0.5 & 376 & 376 & 379 & 377 & 2 \\
\hline & N150 & & & & & & & & & & \\
\hline & $\mathrm{N} 155$ & 10.2 & 9.7 & 9.3 & 9.7 & 0.5 & 354 & 343 & 358 & 352 & 8 \\
\hline & N157 & 9.8 & & & 9.8 & & 356 & & & 356 & \\
\hline & N158 & & & & & & 344 & 325 & 325 & 331 & 11 \\
\hline \multirow{5}{*}{ 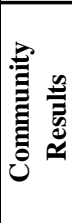 } & & \multicolumn{2}{|c|}{ Consensus Mean } & & 12.6 & & \multicolumn{2}{|c|}{ Consensus Mean } & & 370 & \\
\hline & & \multicolumn{3}{|c|}{ Consensus Standard Deviation } & 4.9 & & \multicolumn{3}{|c|}{ Consensus Standard Deviation } & 33 & \\
\hline & & \multicolumn{3}{|c|}{ Maximum } & 86.8 & & \multicolumn{3}{|c|}{ Maximum } & 447 & \\
\hline & & \multicolumn{3}{|c|}{ Minimum } & 0.0 & & \multicolumn{3}{|c|}{ Minimum } & 0 & \\
\hline & & \multicolumn{3}{|l|}{$\mathrm{N}$} & 16 & & \multicolumn{3}{|l|}{$\mathrm{N}$} & 25 & \\
\hline
\end{tabular}




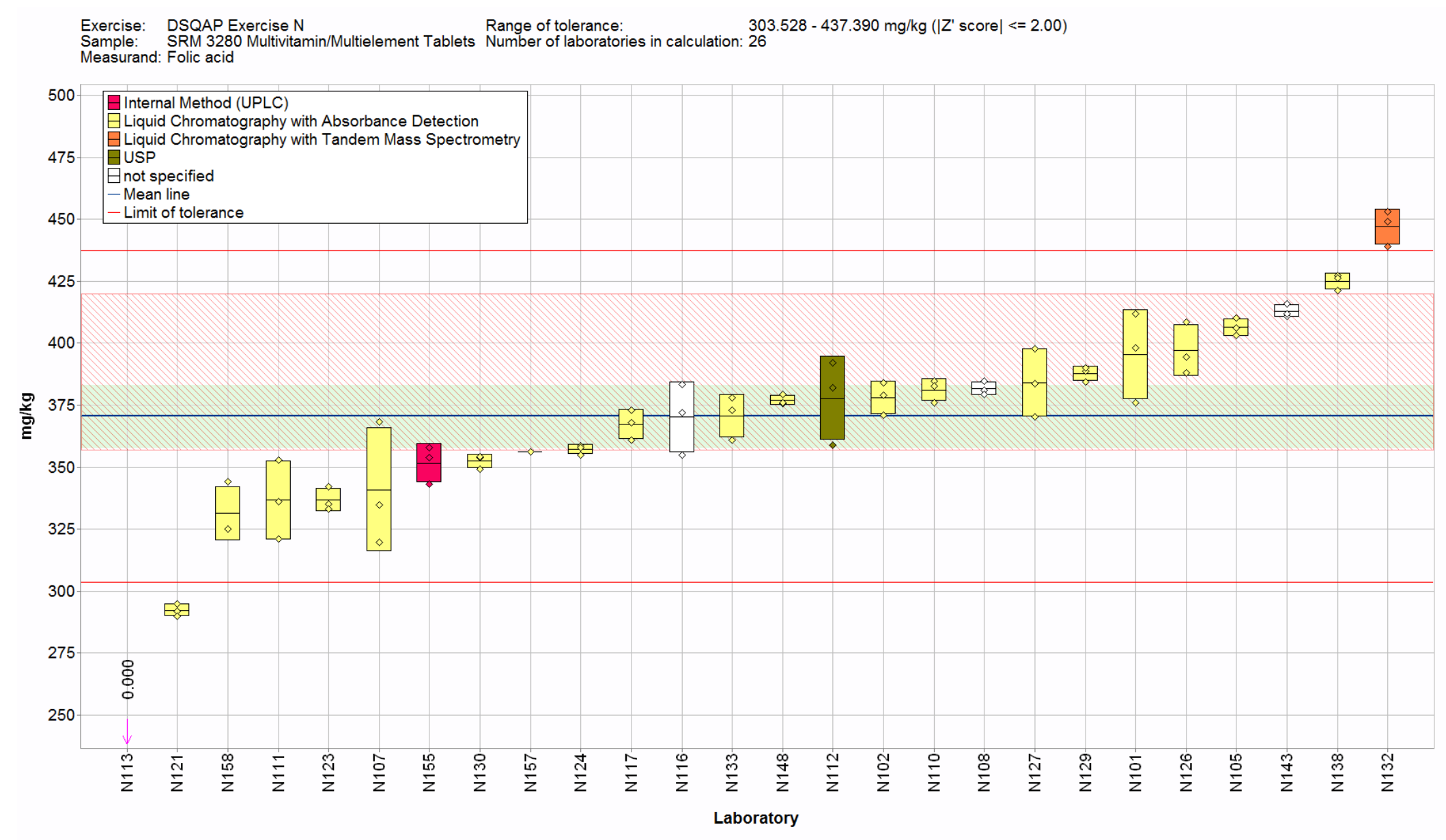

Figure 13. Folic acid in SRM 3280 Multivitamin/Multielement Tablets (data summary view - analytical method). In this view, individual laboratory data are plotted (diamonds) with the individual laboratory standard deviation (rectangle). The color of the data point represents the analytical method employed. The solid blue line represents the consensus mean and the green shaded region represents the $95 \%$ confidence interval for the consensus mean. The solid red lines represent the consensus range of tolerance, calculated as the values above and below the consensus mean that result in an acceptable $Z_{\text {comm }}^{\prime}$ score, $\left|Z_{c o m m}^{\prime}\right| \leq 2$. The red shaded region represents the NIST range of tolerance, which encompasses the NIST certified value bounded by twice its uncertainty (U95) and represents the range that results in an acceptable $Z_{\text {NIST }}$ score, $\left|Z_{\text {NIST }}\right| \leq 2$. 


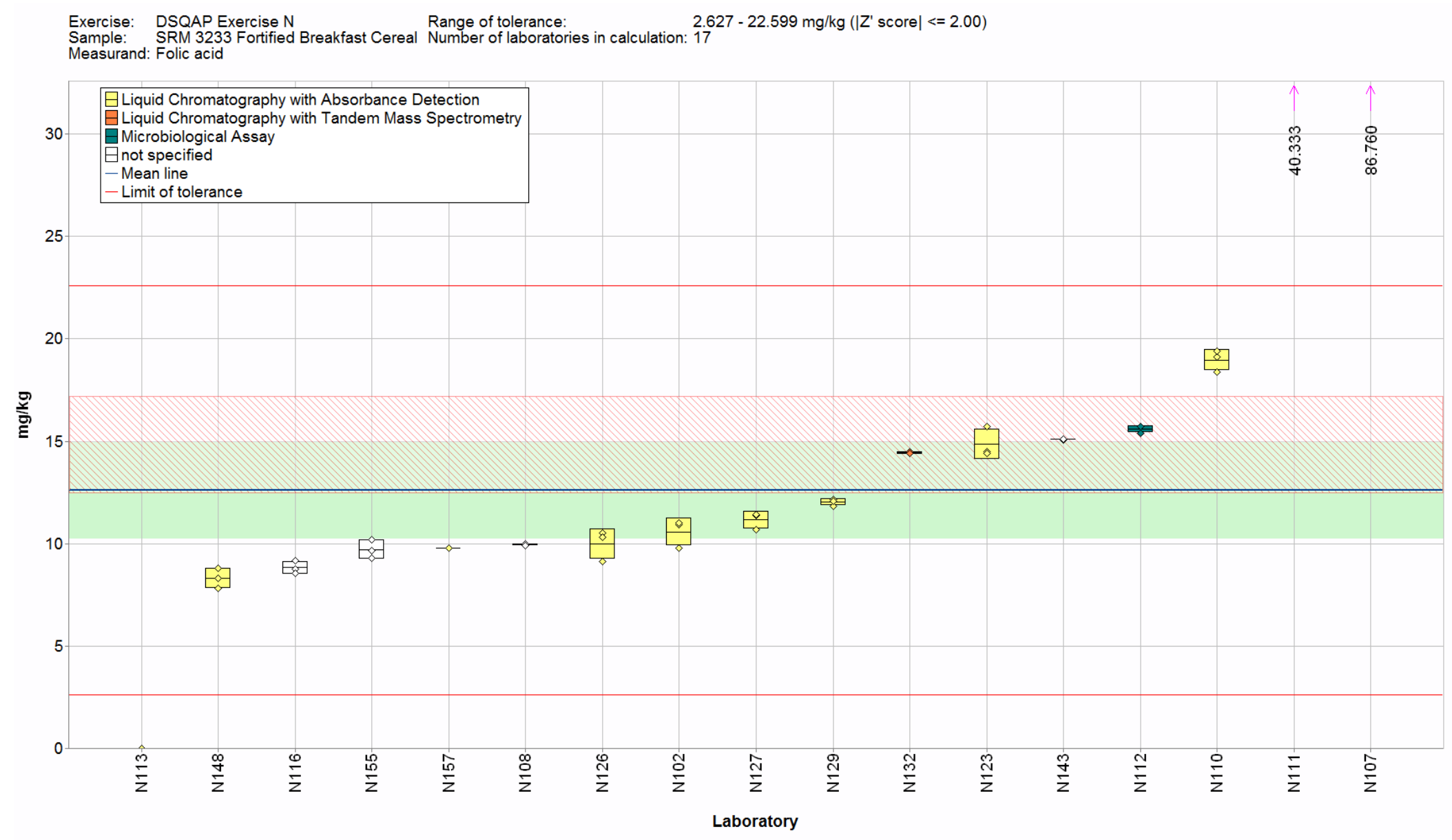

Figure 14. Folic acid in SRM 3233 Fortified Breakfast Cereal (data summary view - analytical method). In this view, individual laboratory data are plotted (diamonds) with the individual laboratory standard deviation (rectangle). The color of the data point represents the analytical method employed. The solid blue line represents the consensus mean and the green shaded region represents the $95 \%$ confidence interval for the consensus mean. The solid red lines represent the consensus range of tolerance, calculated as the values above and below the consensus mean that result in an acceptable $Z_{\text {comm }}^{\prime}$ score, $\left|Z_{\text {comm }}^{\prime}\right| \leq 2$. The red shaded region represents the NIST range of tolerance, which encompasses the NIST certified value bounded by twice its uncertainty (U95) and represents the range that results in an acceptable $Z_{\text {NIST }}$ score, $\left|Z_{\text {NIST }}\right| \leq 2$. 


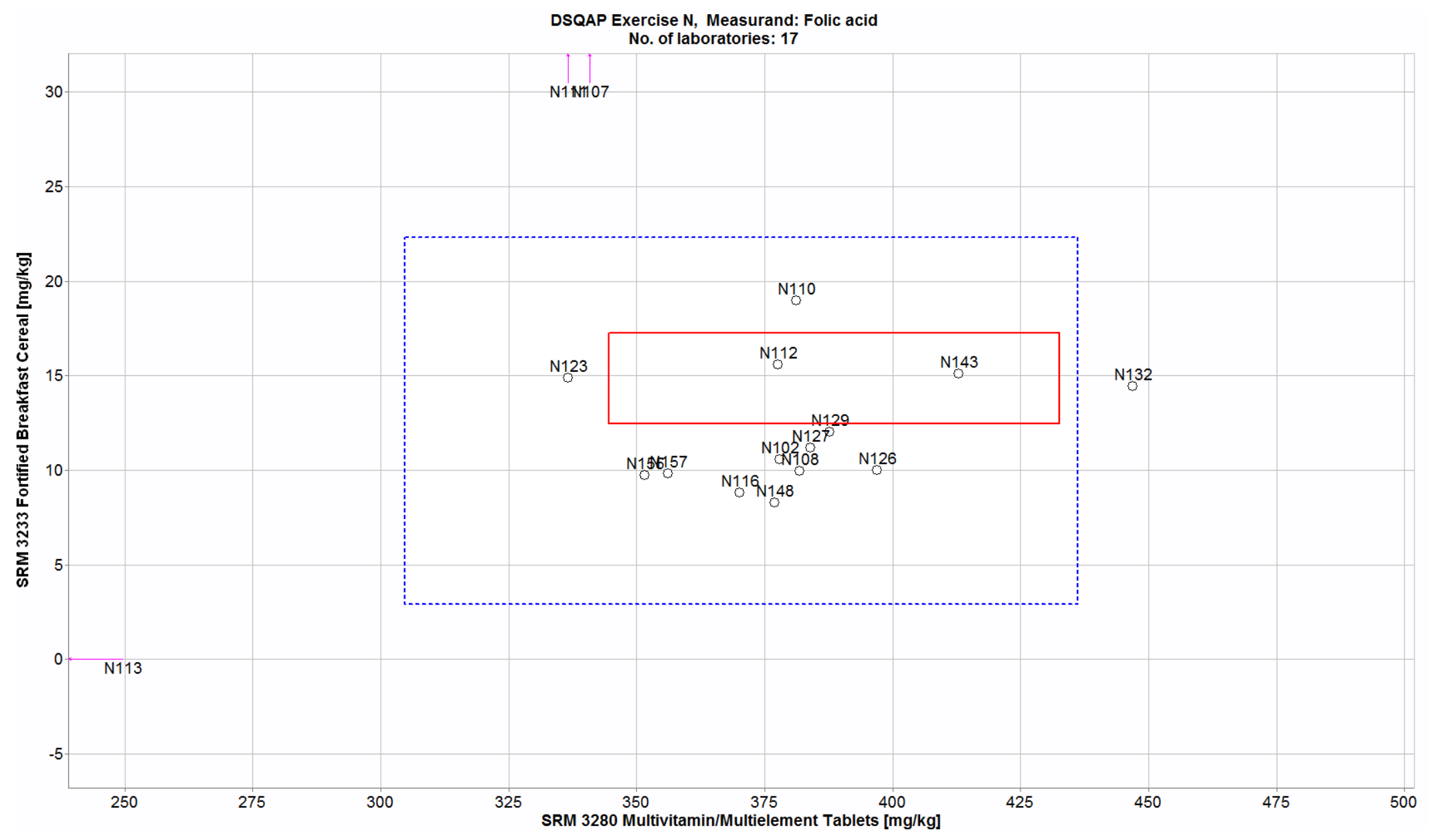

Figure 15. Laboratory means for folic acid in SRM 3280 Multivitamin/Multielement Tablets and SRM 3233 Fortified Breakfast Cereal (sample/sample comparison view). In this view, the individual laboratory mean for one sample (multivitamin) is compared to the mean for a second sample (breakfast cereal). The solid red box represents the NIST range of tolerance for the two samples, multivitamin (x-axis) and breakfast cereal (y-axis), which encompasses the NIST values bounded by their uncertainties (U95) and represents the range that results in an acceptable $Z_{\mathrm{NIST}}$ score, $\left|Z_{\mathrm{NIST}}\right| \leq 2$. The dotted blue box represents the consensus range of tolerance for multivitamin (x-axis) and breakfast cereal (y-axis), calculated as the values above and below the consensus means that result in an acceptable $Z_{c o m m}^{\prime}$ score, $\left|Z_{\text {comm }}^{\prime}\right| \leq 2$. 


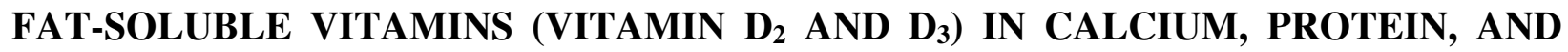 MULTIVITAMIN DIETARY SUPPLEMENTS}

Study Overview

In this study, participants were provided with three NIST SRMs, SRM 3532 Calcium-Containing Solid Oral Dosage Form, SRM 3252 Protein Drink Mix, and SRM 3280 Multivitamin/Multielement Tablets. Participants were asked to use in-house analytical methods to determine the mass fractions of vitamin $\mathrm{D}_{2}$ and vitamin $\mathrm{D}_{3}$ in each of the matrices and report values in $\mathrm{mg} / \mathrm{kg}$ on an as-received basis. SRM 3280 is fortified with vitamin $\mathrm{D}_{2}$ and SRM 3532 is fortified with vitamin $D_{3}$. In addition, the vitamin $\mathrm{D}$ content in the protein drink mix is not fortified.

Sample Information

Calcium Supplement. Participants were provided with three packets, each containing approximately $10 \mathrm{~g}$ of ground calcium supplement. The commercial tablets were ground, homogenized, and heat-sealed inside 4 mil polyethylene bags, which were then sealed inside nitrogen-flushed aluminized plastic bags along with two packets of silica gel. Before use, participants were instructed to thoroughly mix the contents of each packet and use a sample size of at least $1 \mathrm{~g}$. Participants were asked to store the material at controlled room temperature, $10{ }^{\circ} \mathrm{C}$ to $30{ }^{\circ} \mathrm{C}$, and to report a single value from each packet provided. Approximate analyte levels were not reported to participants prior to the study. The reference value for vitamin $\mathrm{D}_{3}$ in SRM 3532 was determined at NIST using isotope dilution liquid chromatography with tandem mass spectrometry detection (ID-LC-MS/MS). A target value for vitamin $\mathrm{D}_{2}$ in SRM 3532 has not been determined at NIST. The NIST value and uncertainty for vitamin $\mathrm{D}_{3}$ is provided in the table below, both on an as-received basis and on a dry-mass basis, accounting for moisture of the material $(3.2 \%)$.

$$
\text { Reference Mass Fraction in SRM } 3532 \text { (mg/kg) }
$$

\begin{tabular}{cccccc}
$\underline{\text { Analyte }}$ & \multicolumn{2}{c}{$\frac{\text { (dry-mass basis })}{\text { (as-received basis) }}$} \\
Vitamin $\mathrm{D}_{3}$ & $1.310 \pm 0.033$ & & $1.268 \pm 0.032$
\end{tabular}

Protein Supplement. Participants were provided with three packets, each containing approximately $10 \mathrm{~g}$ of protein powder. The commercial protein powder was ground, homogenized, and heat-sealed inside 4 mil polyethylene bags, which were then sealed inside nitrogen-flushed aluminized plastic bags along with two packets of silica gel. Before use, participants were instructed to thoroughly mix the contents of each packet and to use a sample size of at least $2 \mathrm{~g}$. Participants were asked to store the material at controlled room temperature, $10{ }^{\circ} \mathrm{C}$ to $30{ }^{\circ} \mathrm{C}$, and to prepare a single sample and to report a single value from each packet provided. Approximate analyte levels were not reported to participants prior to the study, and target values for these analytes in SRM 3252 have not been determined at NIST.

Multivitamin. Participants were provided with one bottle containing 30 multivitamin/multielement tablets. Before use, participants were instructed to grind all tablets, mix the resulting powder thoroughly, and to use a sample size of at least $0.6 \mathrm{~g}$. Participants were asked to store the material at controlled room temperature, $10^{\circ} \mathrm{C}$ to $30^{\circ} \mathrm{C}$, and to prepare three samples and report three values from the single bottle provided. The approximate analyte level was not reported to 
participants prior to the study. The reference value for vitamin $\mathrm{D}_{2}$ in $\mathrm{SRM} 3280$ was determined at NIST using ID-LC-MS. A target value for vitamin $\mathrm{D}_{3}$ in SRM 3280 has not been determined at NIST. The reference value and uncertainty for vitamin $\mathrm{D}_{2}$ is provided in the table below, both on a dry-mass basis and on an as-received basis accounting for moisture of the material $(1.37 \%)$.

$\begin{array}{ccc}\stackrel{\text { Analyte }}{\text { Vitamin } \mathrm{D}_{2}} & \frac{\text { (dry-mass basis) }}{8.6 \pm 2.6} & \text { (as-received basis) } \\ 8.5 \pm 2.6\end{array}$

Study Results

- Twenty-nine laboratories enrolled in this exercise and received samples to measure vitamin $\mathrm{D}_{2}$ in dietary supplements. Two laboratories reported results for vitamin $\mathrm{D}_{2}$ in the calcium supplement (7\% participation), seven laboratories reported results for vitamin $\mathrm{D}_{2}$ in the protein supplement, (24\% participation) and thirteen laboratories reported results for vitamin $\mathrm{D}_{2}$ in the multivitamin (45\% participation).

- The consensus mean was within the target range for vitamin $\mathrm{D}_{2}$ in the multivitamin.

- The between-laboratory variability ranged from very high in the multivitamin $(41 \%)$ to unacceptable in the calcium supplement and the protein supplement (>100\% and $98 \%$, respectively).

- Laboratories reported using LC-absorbance (50\% to $60 \%$ of laboratories) and LC-MS/MS (14\% to $20 \%$ of laboratories) as their analytical approach. Some laboratories did not report the analytical method used.

- Thirty-three laboratories enrolled in this exercise and received samples to measure vitamin $\mathrm{D}_{3}$ in dietary supplements. Nineteen laboratories reported results for vitamin $\mathrm{D}_{3}$ in the calcium supplement (58\% participation) and seven laboratories reported results for vitamin $\mathrm{D}_{3}$ in both the protein supplement and the multivitamin (21\% participation).

- The consensus mean was below the target range for vitamin $\mathrm{D}_{3}$ in the protein supplement and the consensus range slightly overlapped the target range.

- The between-laboratory variability ranged from moderate in the calcium supplement $(25 \%)$ to very high in the multivitamin $(71 \%)$. The between-laboratory variability was unacceptable in the protein supplement (>100\%).

- Laboratories reported using LC-absorbance (58\% to $67 \%$ of laboratories) and LC-MS/MS (10\% to $16 \%$ of laboratories) as their analytical approach. Some laboratories did not report the analytical method used.

Technical Recommendations

The following recommendations are based on results obtained from the participants in this study.

- No analytical method was identified as exceptionally good or problematic. LC-MS/MS and LC-absorbance methods gave very similar results for samples of this type in these concentration ranges.

- Each of these samples only contains one form of vitamin $\mathrm{D}$ (either vitamin $\mathrm{D}_{2}$ or vitamin $\mathrm{D}_{3}$, but not both). In addition, the vitamin $\mathrm{D}$ content in the protein drink mix is not fortified. As a result, many parts of this study yielded low participation rates or many laboratories indicated that the analyte was below their limit of detection. 
- Focusing only on the analyte/matrix pairs that are fortified, the data variability was acceptable. For both vitamin $\mathrm{D}_{2}$ in the multivitamin and vitamin $\mathrm{D}_{3}$ in the calcium supplement, the spread of the consensus range was comparable to or smaller than the NIST target range.

- Because all sample preparation methods are not appropriate for all matrices, some in-house optimization may be necessary when analyzing a new material to confirm that the procedure is appropriate. Because the form of vitamin $\mathrm{D}$ present in each material was not specified, all materials should have been screened for vitamin $\mathrm{D}_{2}$ and vitamin $\mathrm{D}_{3}$.

- For both vitamin $\mathrm{D}_{2}$ in the multivitamin and vitamin $\mathrm{D}_{3}$ in the calcium supplement, some laboratories reported values that were high for both sample/analyte pairs (or low for both).

- The low measured values may be a result of incomplete extraction of the vitamin $\mathrm{D}$ from the sample matrix. To ensure maximum recovery, a subsequent re-extraction of the sample can indicate if measurable amounts of the analyte remain in the matrix following extraction.

- The high measured values may indicate chromatographic coelutions with matrix components. Because each matrix is different, the chromatographic method should be evaluated to confirm any potential biases resulting from interfering compounds.

- Due to the potential for contamination in calibration materials, NIST typically determines the concentration of calibration solutions for certain analytes, including vitamin D, with a spectrophotometer and then adjusts for LC purity at the same wavelength. Impurities in calibration materials could be a source of bias when laboratory-determined values are compared to NIST certified values. 
Table 9. Individualized data summary table (NIST) for ergocalciferol (vitamin $\mathrm{D}_{2}$ ) and cholecalciferol (vitamin $\mathrm{D}_{3}$ ) in dietary supplements.

\section{National Institute of Standards \& Technology}

\begin{tabular}{|c|c|c|c|c|c|c|c|c|c|c|c|}
\hline \multirow[b]{2}{*}{ Analyte } & \multirow{2}{*}{$\begin{array}{l}\text { Lab Code: } \\
\text { Sample } \\
\end{array}$} & \multirow{2}{*}{$\begin{array}{l}\text { NIST } \\
\text { Units } \\
\end{array}$} & \multicolumn{4}{|c|}{$\begin{array}{c}\text { Exercise N - May } 2017 \text { - Vitamin D } \\
\text { 1. Your Results }\end{array}$} & \multicolumn{3}{|c|}{ 2. Community Results } & \multicolumn{2}{|c|}{ 3. Target } \\
\hline & & & $\mathrm{x}_{\mathrm{i}}$ & $\mathrm{s}_{\mathrm{i}}$ & $Z_{\text {comm }}^{\prime}$ & $Z_{\text {NIST }}$ & $\mathrm{N}$ & $\mathrm{x}^{*}$ & $\mathrm{~s}^{*}$ & $\mathrm{x}_{\mathrm{NIST}}$ & $U_{95}$ \\
\hline Ergocalciferol (Vitamin $\left.\mathrm{D}_{2}\right)$ & Protein Drink & $\mathrm{mg} / \mathrm{kg}$ & & & & & 7 & 3.12 & 5.31 & & \\
\hline Ergocalciferol (Vitamin $\left.D_{2}\right)$ & Ca Supplement & $\mathrm{mg} / \mathrm{kg}$ & & & & & 2 & 6.85 & 6.68 & & \\
\hline Ergocalciferol (Vitamin $\left.\mathrm{D}_{2}\right)$ & Multivitamin & $\mathrm{mg} / \mathrm{kg}$ & 8.5 & 2.6 & & 0.00 & 13 & 7.95 & 3.24 & 8.5 & 2.6 \\
\hline Cholecalciferol (Vitamin $D_{3}$ ) & Protein Drink & $\mathrm{mg} / \mathrm{kg}$ & & & & & 7 & 0.51 & 0.56 & & \\
\hline Cholecalciferol (Vitamin $D_{3}$ ) & Ca Supplement & $\mathrm{mg} / \mathrm{kg}$ & 1.27 & 0.03 & & 0.00 & 19 & 1.12 & 0.28 & 1.27 & 0.03 \\
\hline Cholecalciferol (Vitamin $D_{3)}$ & Multivitamin & $\mathrm{mg} / \mathrm{kg}$ & & & & & 7 & 6.49 & 4.59 & & \\
\hline
\end{tabular}

$\mathrm{x}_{\mathrm{i}}$ Mean of reported values

$\mathrm{s}_{\mathrm{i}}$ Standard deviation of reported values

$\mathrm{Z}_{\text {comm }}^{\prime} \mathrm{Z}^{\prime}$-score with respect to communit consensus

$\mathrm{x}_{\mathrm{i}}$ Mean of reported values
$\mathrm{N}$ Number of quantitative values reported

$\mathrm{x}^{*}$ Robust mean of reported values

$\mathrm{N}$ Number of quantitative $\mathrm{x}_{\text {NIST }}$ NIST-assessed value $U_{95} \pm 95 \%$ confidence interval about the assessed value or standard deviation $\left(\mathrm{s}_{\mathrm{NIST}}\right)$

$\mathrm{x}_{\text {NIST }}$ NIST-assessed value 
Table 10. Data summary table for ergocalciferol (vitamin $D_{2}$ ) in dietary supplements. Data highlighted in red have been flagged as potential outliers (e.g., difference from reference value, Grubb and/or Cochran) by the NIST software package.

\begin{tabular}{|c|c|c|c|c|c|c|c|c|c|c|c|c|c|c|c|c|}
\hline & \multirow[b]{3}{*}{ Lab } & \multicolumn{15}{|c|}{ Ergocalciferol (Vitamin $D_{2}$ ) } \\
\hline & & \multicolumn{5}{|c|}{ SRM 3252 Protein Drink Mix (mg/kg) } & \multicolumn{5}{|c|}{ SRM 3532 Calcium Dietary Supplement $(\mathrm{mg} / \mathrm{kg})$} & \multicolumn{5}{|c|}{ SRM 3280 Multivitamin/Multielement Tablets ( $\mathrm{mg} / \mathrm{kg}$ ) } \\
\hline & & $\mathbf{A}$ & $\mathbf{B}$ & $\mathbf{C}$ & Avg & SD & $\mathbf{A}$ & $\mathbf{B}$ & $\mathbf{C}$ & Avg & SD & $\mathbf{A}$ & $\mathbf{B}$ & $\mathbf{C}$ & Avg & SD \\
\hline \multirow{30}{*}{ 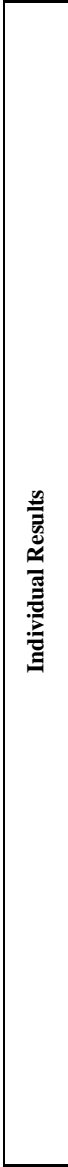 } & NIST & & & & & & & & & & & & & & 8.5 & 2.6 \\
\hline & N101 & & & & & & $<0.003$ & $<0.003$ & $<0.003$ & $<0.003$ & & 7.40 & 6.70 & 5.50 & 6.53 & 0.96 \\
\hline & N102 & & & & & & & & & & & & & & & \\
\hline & N105 & & & & & & & & & & & & & & & \\
\hline & N107 & & & & & & & & & & & 1.55 & 1.65 & 2.15 & 1.78 & 0.32 \\
\hline & N108 & 9.00 & 8.61 & 8.50 & 8.70 & 0.26 & & & & & & 8.61 & 8.54 & 8.65 & 8.60 & 0.06 \\
\hline & N110 & 0.50 & 0.80 & 0.50 & 0.60 & 0.17 & 3.50 & 2.90 & 1.70 & 2.70 & 0.92 & 20.80 & 18.50 & 30.00 & 23.10 & 6.09 \\
\hline & N111 & & & & & & 11.00 & 10.00 & 12.00 & 11.00 & 1.00 & & & & & \\
\hline & N112 & 1.40 & 0.35 & 0.35 & 0.70 & 0.61 & $<0.001$ & $<0.001$ & $<0.001$ & $<0.001$ & & 8.87 & 8.53 & 8.72 & 8.71 & 0.17 \\
\hline & N113 & $<0.00$ & $<0.00$ & $<0.00$ & $<0.00$ & & $<0.00$ & $<0.00$ & $<0.00$ & $<0.00$ & & $<0.000$ & $<0.000$ & $<0.000$ & $<0.000$ & \\
\hline & N115 & & & & & & & & & & & & & & & \\
\hline & N116 & & & & & & & & & & & 6.20 & 5.82 & 6.11 & 6.05 & 0.20 \\
\hline & N120 & & & & & & & & & & & & & & & \\
\hline & $\mathrm{N} 122$ & & & & & & & & & & & & & & & \\
\hline & N124 & & & & & & & & & & & 7.96 & 9.44 & 8.61 & 8.67 & 0.74 \\
\hline & N125 & & & & & & & & & & & & & & & \\
\hline & N126 & & & & & & & & & & & & & & & \\
\hline & N127 & 13.60 & 14.10 & 14.40 & 14.03 & 0.40 & & & & & & & & & & \\
\hline & N129 & & & & & & & & & & & & & & & \\
\hline & N130 & & & & & & & & & & & & & & & \\
\hline & $\mathrm{N} 132$ & 0.19 & 0.20 & 0.21 & 0.20 & 0.01 & $<0.10$ & $<0.10$ & $<0.10$ & $<0.10$ & & 7.48 & 8.18 & 8.32 & 7.99 & 0.45 \\
\hline & N133 & & & & & & & & & & & 6.36 & 6.17 & 6.41 & 6.31 & 0.13 \\
\hline & N134 & 0.30 & 0.28 & 0.34 & 0.31 & 0.03 & $<0.20$ & $<0.20$ & $<0.20$ & $<0.20$ & & 10.91 & 9.79 & 10.40 & 10.37 & 0.56 \\
\hline & N141 & & & & & & & & & & & & & & & \\
\hline & N143 & & & & & & & & & & & 12.74 & 12.73 & 12.56 & 12.68 & 0.10 \\
\hline & N147 & & & & & & & & & & & & & & & \\
\hline & N149 & & & & & & & & & & & & & & & \\
\hline & N155 & $<0.25$ & $<0.25$ & $<0.25$ & $<0.25$ & & $<0.25$ & $<0.25$ & $<0.25$ & $<0.25$ & & 5.24 & 3.14 & 5.60 & 4.66 & 1.33 \\
\hline & N157 & & & & & & & & & & & & & & & \\
\hline & N158 & 0.18 & 0.30 & 0.20 & 0.23 & 0.06 & & & & & & 7.59 & 6.00 & 7.01 & 6.87 & 0.80 \\
\hline \multirow{5}{*}{ 象 } & & \multicolumn{3}{|c|}{ Consensus Mean } & \multicolumn{2}{|l|}{3.12} & \multicolumn{2}{|c|}{ Consensus Mean } & & 6.85 & & \multicolumn{2}{|c|}{ Consensus Mean } & & 7.95 & \\
\hline & & \multicolumn{3}{|c|}{ Consensus Standard Deviation } & \multicolumn{2}{|l|}{5.31} & \multicolumn{3}{|c|}{ Consensus Standard Deviation } & 6.68 & & \multicolumn{3}{|c|}{ Consensus Standard Deviation } & 3.24 & \\
\hline & & \multirow{2}{*}{\multicolumn{3}{|c|}{ Maximum }} & \multicolumn{2}{|l|}{14.03} & \multicolumn{3}{|c|}{ Maximum } & 11.00 & & \multicolumn{3}{|l|}{ Maximum } & 23.10 & \\
\hline & & \multirow{2}{*}{\multicolumn{3}{|c|}{$\begin{array}{l}\text { Minimum } \\
\mathrm{N}\end{array}$}} & \multicolumn{2}{|l|}{0.20} & Minimum & & & 2.70 & & Minimum & & & 1.78 & \\
\hline & & & & & 7 & & $\mathrm{~N}$ & & & 2 & & $\mathrm{~N}$ & & & 13 & \\
\hline
\end{tabular}


Table 11. Data summary table for cholecalciferol (vitamin $\mathrm{D}_{3}$ ) in dietary supplements. Data highlighted in red have been flagged as potential outliers (e.g., difference from reference value, Grubb and/or Cochran) by the NIST software package.

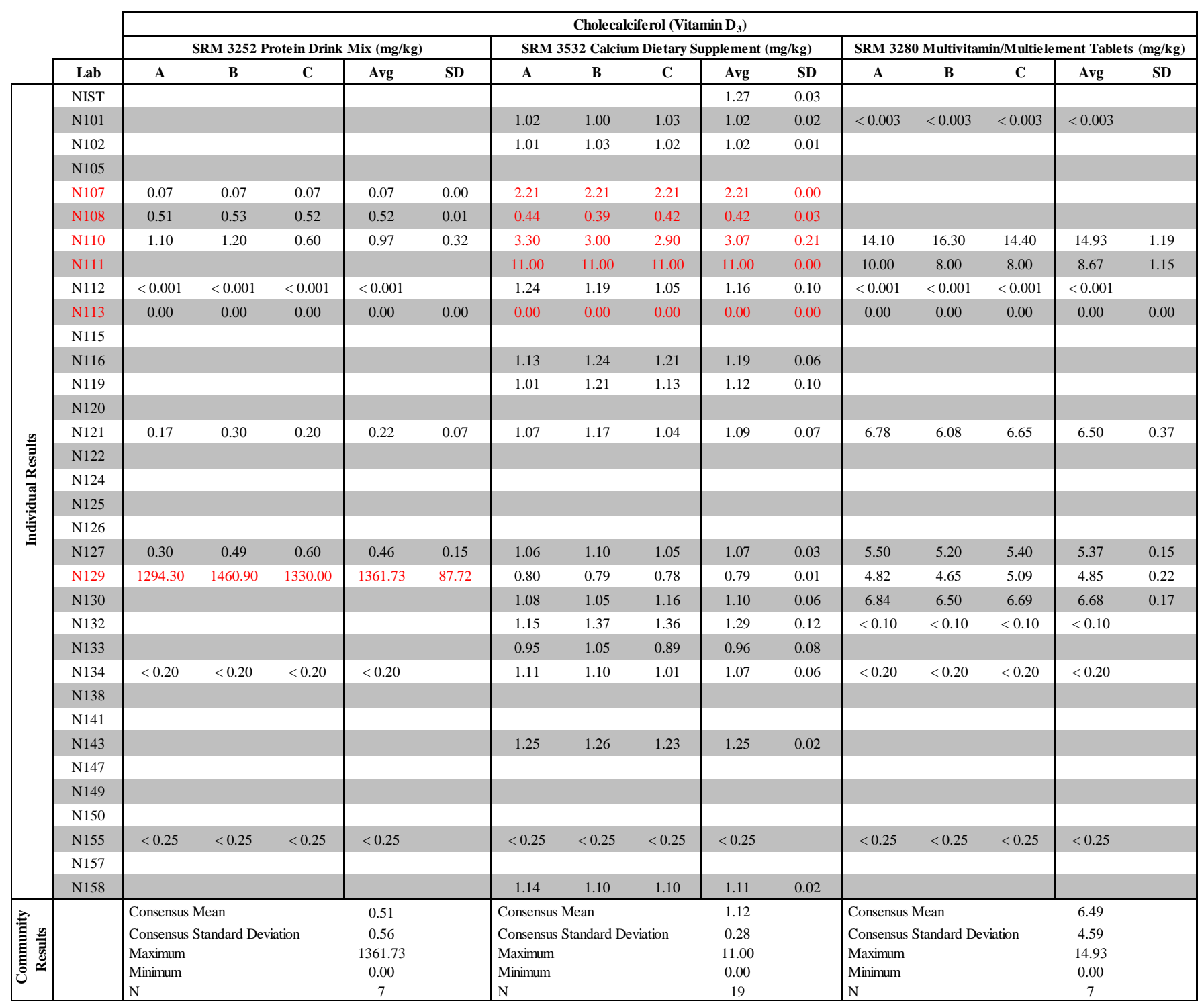




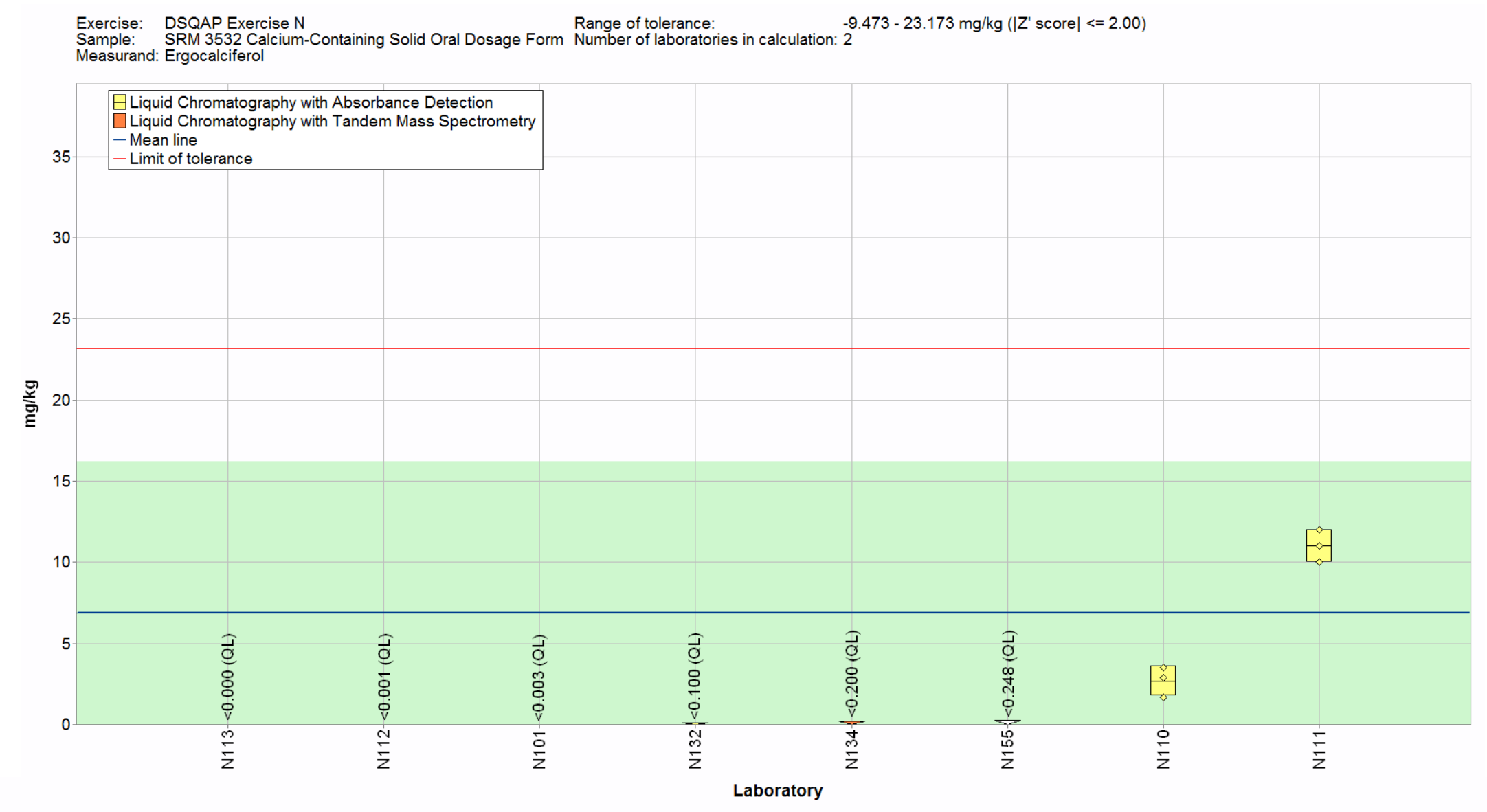

Figure 16. Total ergocalciferol (vitamin $D_{2}$ ) in SRM 3532 Calcium-Containing Solid Oral Dosage Form (data summary view analytical method). In this view, individual laboratory data are plotted (diamonds) with the individual laboratory standard deviation (rectangle). The color of the data point represents the analytical method employed. Laboratory data shown as a triangle indicates that a "less than" result was submitted, and the base of the triangle is displayed at the reported laboratory detection limit. The solid blue line represents the consensus mean and the green shaded region represents the $95 \%$ confidence interval for the consensus mean. The solid red line represents the upper consensus range of tolerance, calculated as the values above and below the consensus mean that result in an acceptable $Z_{\text {comm }}^{\prime}$ score, $\left|Z_{\text {comm }}^{\prime}\right| \leq 2$, with the lower range set at zero. A NIST value has not been determined in this material. 


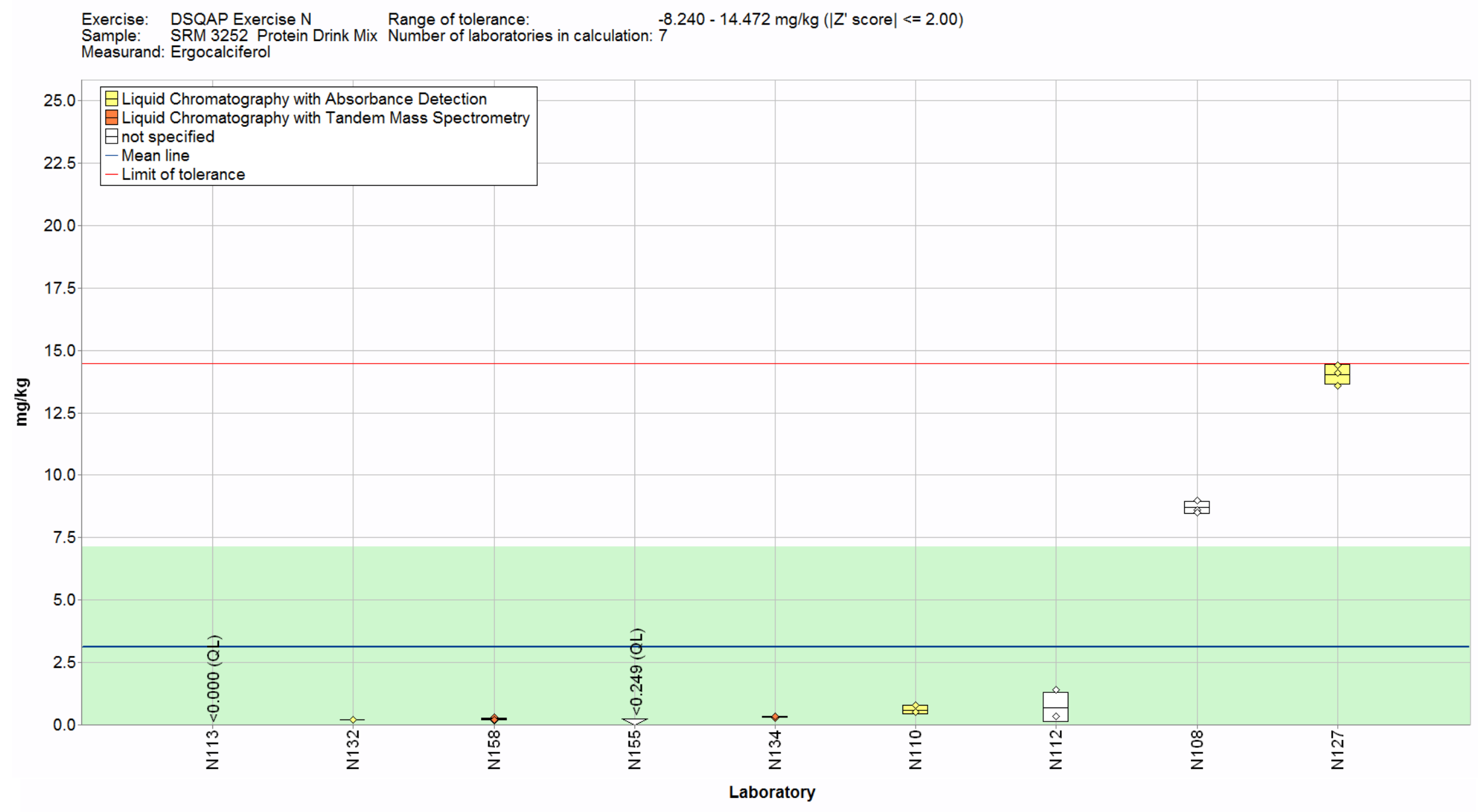

Figure 17. Total ergocalciferol (vitamin $\mathrm{D}_{2}$ ) in SRM 3252 Protein Drink Mix (data summary view - analytical method). In this view, individual laboratory data are plotted (diamonds) with the individual laboratory standard deviation (rectangle). The color of the data point represents the analytical method employed. Laboratory data shown as a triangle indicates that a "less than" result was submitted, and the base of the triangle is displayed at the reported laboratory detection limit. The solid blue line represents the consensus mean and the green shaded region represents the $95 \%$ confidence interval for the consensus mean. The solid red line represents the upper consensus range of tolerance, calculated as the values above and below the consensus mean that result in an acceptable $Z_{c o m m}^{\prime}$ score, $\left|Z_{\text {comm }}^{\prime}\right| \leq 2$, with the lower range set at zero. A NIST value has not been determined in this material. 


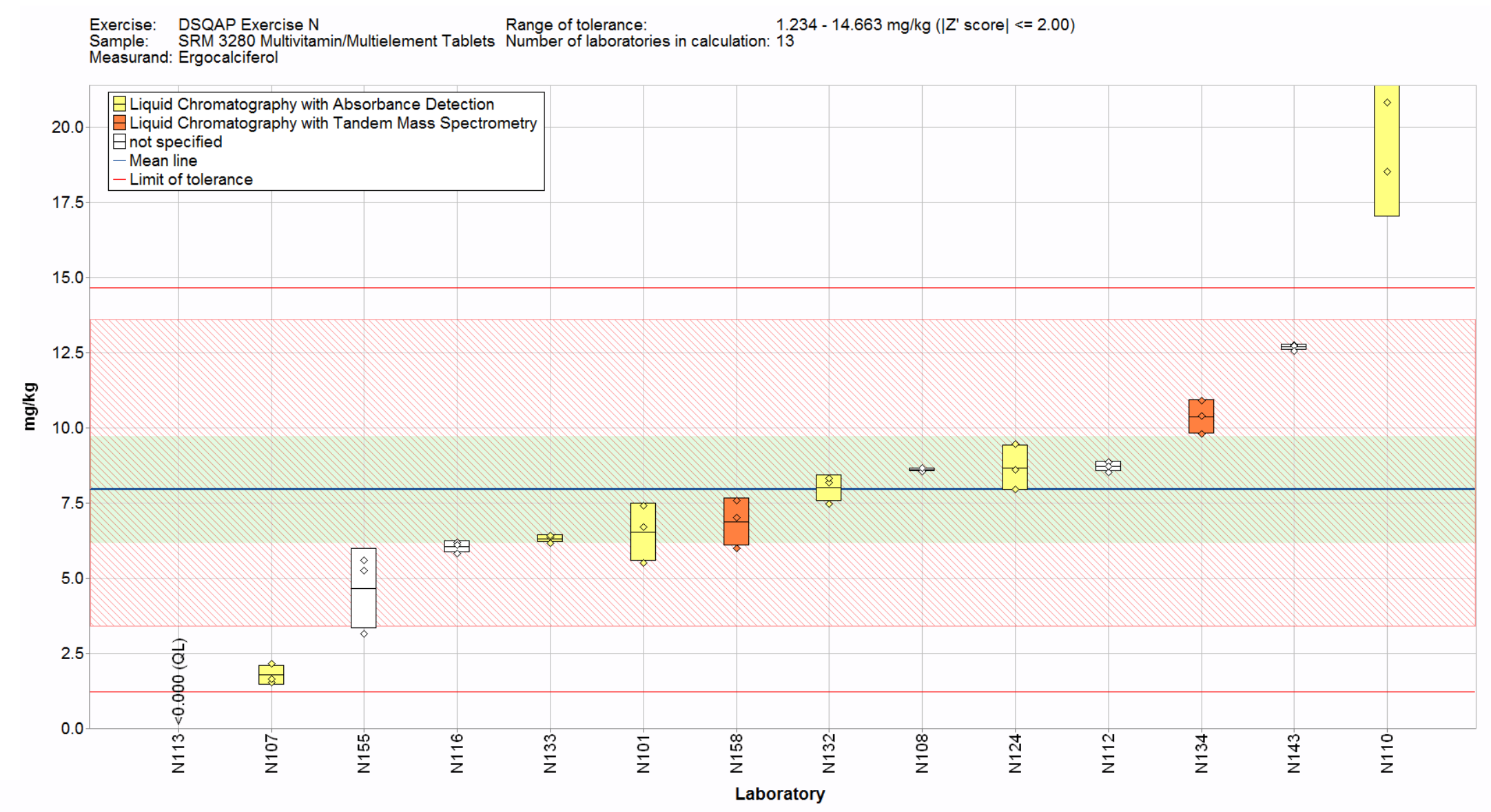

Figure 18. Total ergocalciferol (vitamin $D_{2}$ ) in SRM 3280 Multivitamin/Multielement Tablets (data summary view - analytical method). In this view, individual laboratory data are plotted (diamonds) with the individual laboratory standard deviation (rectangle). The color of the data point represents the analytical method employed. Laboratory data shown as a triangle indicates that a "less than" result was submitted, and the base of the triangle is displayed at the reported laboratory detection limit. The solid blue line represents the consensus mean and the green shaded region represents the $95 \%$ confidence interval for the consensus mean. The solid red lines represent the consensus range of tolerance, calculated as the values above and below the consensus mean that result in an acceptable $Z_{\text {comm }}^{\prime}$ score, $\left|Z_{\text {comm }}^{\prime}\right| \leq 2$. The red shaded region represents the NIST range of tolerance, which encompasses the NIST reference value bounded by twice its uncertainty $\left(U_{95}\right)$ and represents the range that results in an acceptable $Z_{\text {NIST }}$ score, $\left|Z_{\text {NIST }}\right| \leq 2$. 


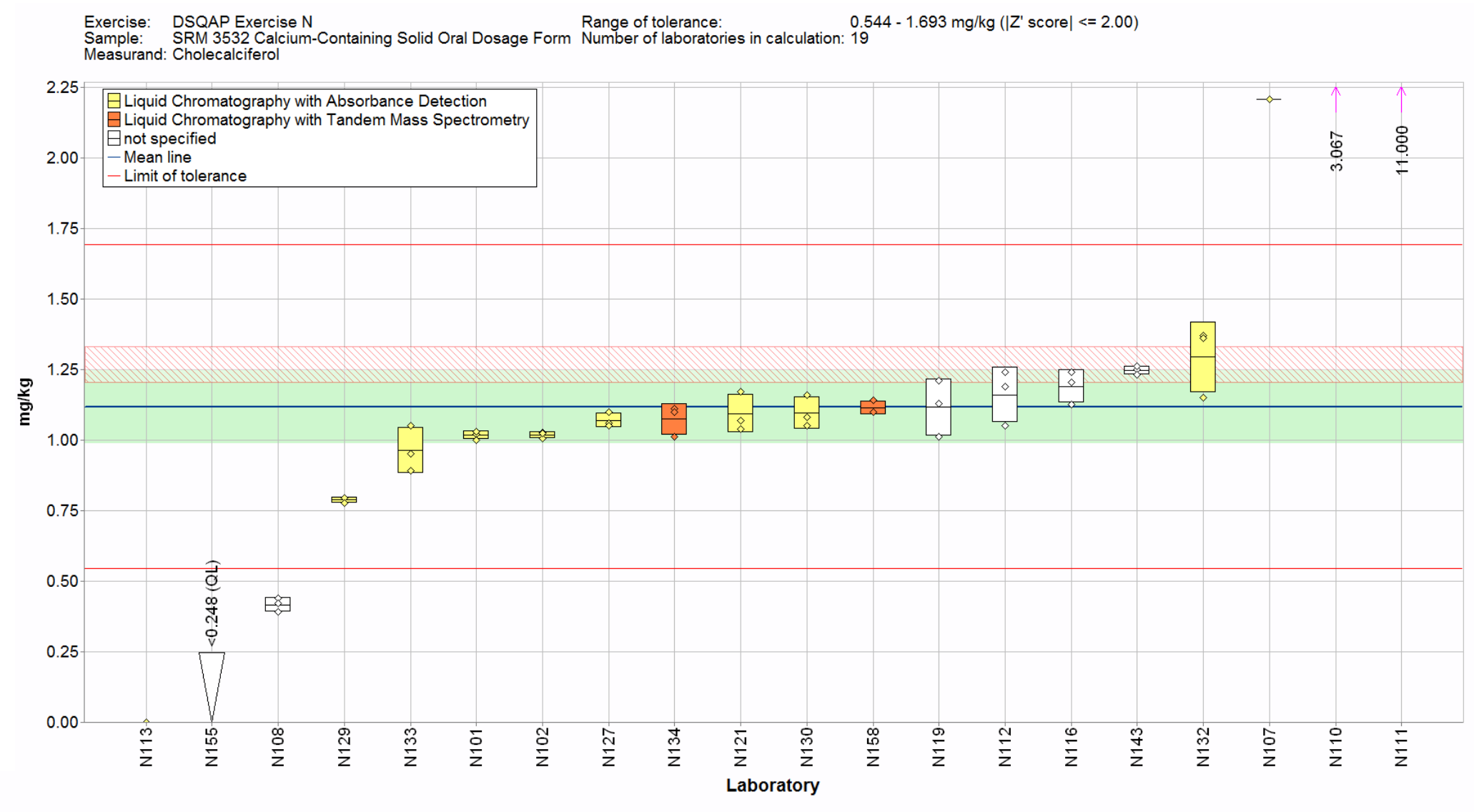

Figure 19. Total cholecalciferol (vitamin $\mathrm{D}_{3}$ ) in SRM 3532 Calcium-Containing Solid Oral Dosage Form (data summary view analytical method). In this view, individual laboratory data are plotted (diamonds) with the individual laboratory standard deviation (rectangle). The color of the data point represents the analytical method employed. Laboratory data shown as a triangle indicates that a "less than" result was submitted, and the base of the triangle is displayed at the reported laboratory detection limit. The solid blue line represents the consensus mean and the green shaded region represents the $95 \%$ confidence interval for the consensus mean. The solid red lines represent the consensus range of tolerance, calculated as the values above and below the consensus mean that result in an acceptable $Z_{\text {comm }}^{\prime}$ score, $\left|Z_{\text {comm }}^{\prime}\right| \leq 2$. The red shaded region represents the NIST range of tolerance, which encompasses the NIST certified value bounded by twice its uncertainty $\left(U_{95}\right)$ and represents the range that results in an acceptable $Z_{\text {NIST }}$ score, $\left|Z_{\text {NIST }}\right| \leq 2$. 


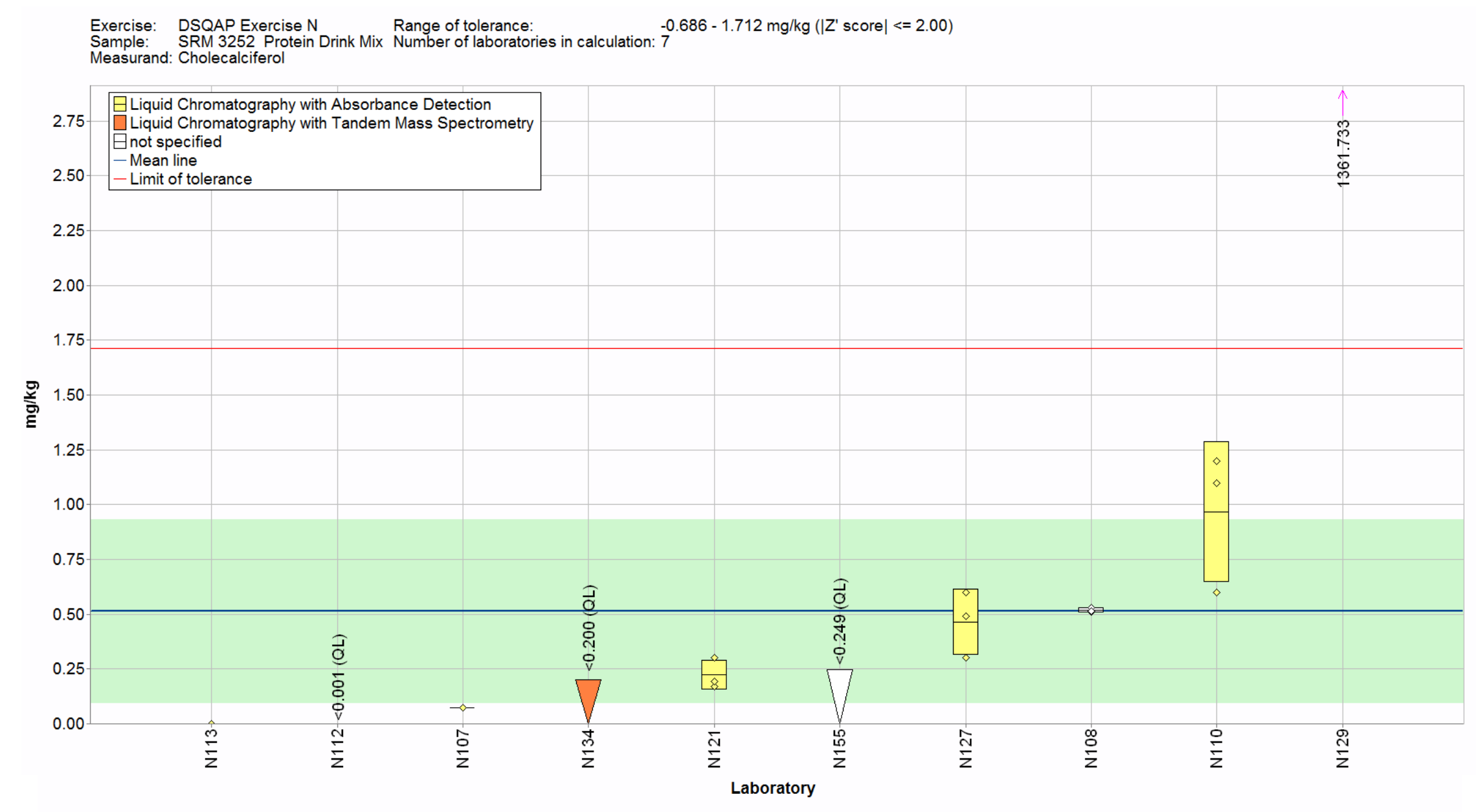

Figure 20. Total cholecalciferol (vitamin $D_{3}$ ) in SRM 3252 Protein Drink Mix (data summary view - analytical method). In this view, individual laboratory data are plotted (diamonds) with the individual laboratory standard deviation (rectangle). The color of the data point represents the analytical method employed. Laboratory data shown as a triangle indicates that a "less than" result was submitted, and the base of the triangle is displayed at the reported laboratory detection limit. The solid blue line represents the consensus mean and the green shaded region represents the $95 \%$ confidence interval for the consensus mean. The solid red line represents the upper consensus range of tolerance, calculated as the values above and below the consensus mean that result in an acceptable $Z_{\text {comm }}^{\prime}$ score, $\left|Z_{c o m m}^{\prime}\right| \leq 2$, with the lower range set at zero. A NIST value has not been determined in this material. 


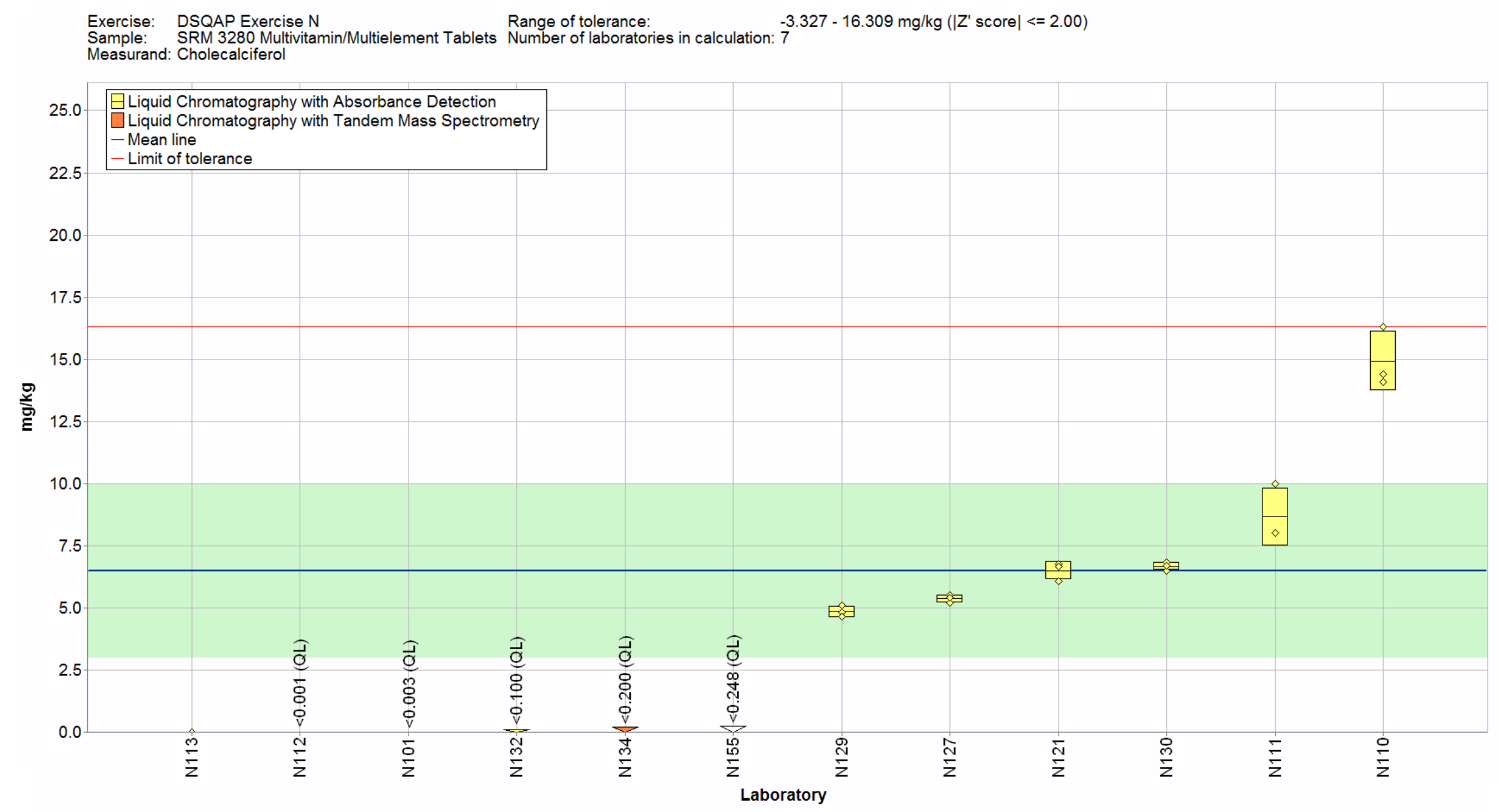

Figure 21. Total cholecalciferol (vitamin D3) in SRM 3280 Multivitamin/Multielement Tablets (data summary view - analytical method). In this view, individual laboratory data are plotted (diamonds) with the individual laboratory standard deviation (rectangle). The color of the data point represents the analytical method employed. Laboratory data shown as a triangle indicates that a "less than" result was submitted, and the base of the triangle is displayed at the reported laboratory detection limit. The solid blue line represents the consensus mean and the green shaded region represents the $95 \%$ confidence interval for the consensus mean. The solid red line represents the upper consensus range of tolerance, calculated as the values above and below the consensus mean that result in an acceptable $Z_{\text {comm }}^{\prime}$ score, $\left|Z_{\text {comm }}^{\prime}\right| \leq 2$, with the lower range set at zero. A NIST value has not been determined in this material. 
DSQAP Exercise N, Measurand: Ergocalciferol

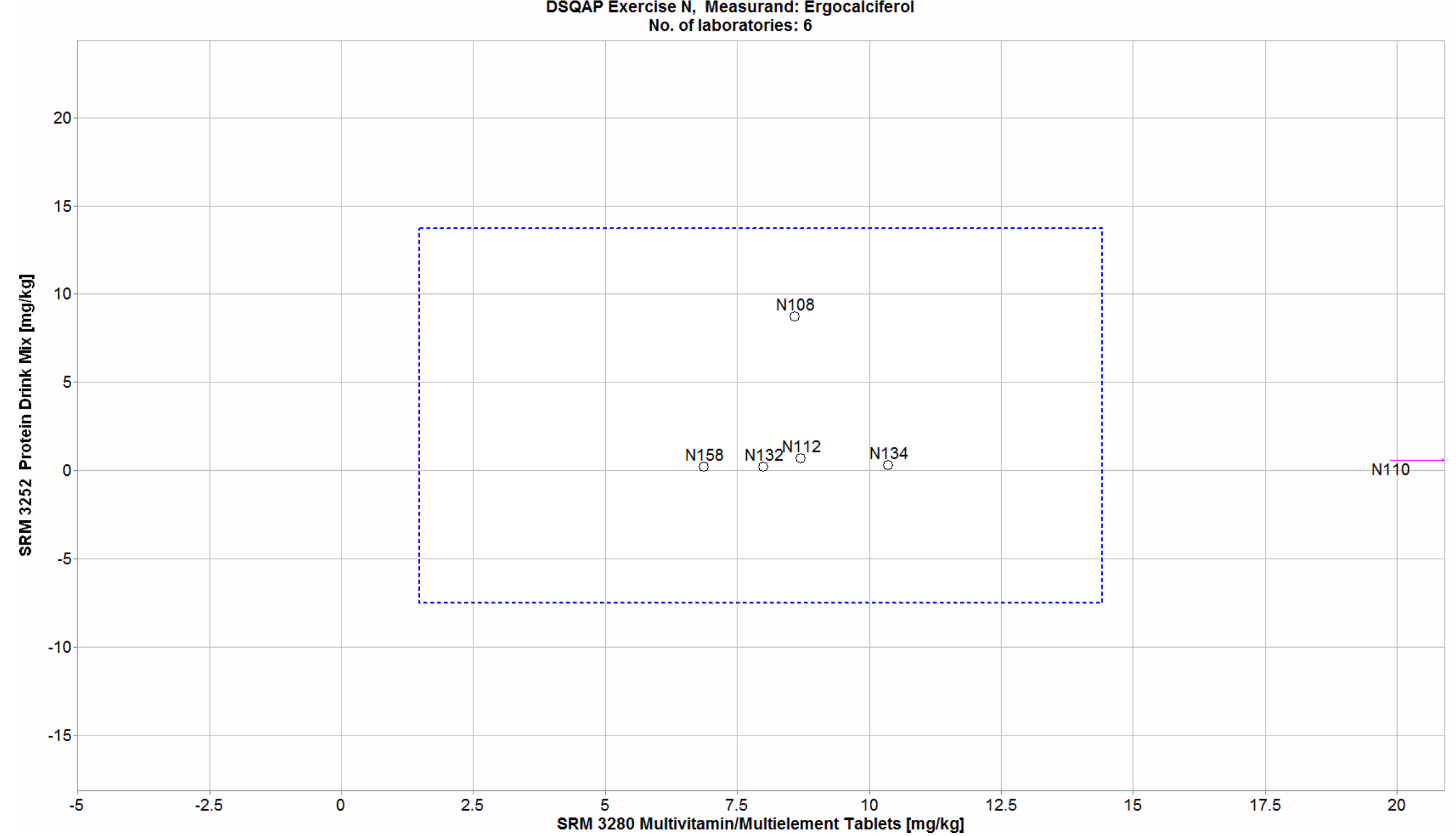

Figure 22. Laboratory means for ergocalciferol (vitamin $\mathrm{D}_{2}$ ) in SRM 3280 Multivitamin/Multielement Tablets and SRM 3252 Protein Drink Mix (sample/sample comparison view). In this view, the individual laboratory mean for one sample (multivitamin) is compared to the mean for a second sample (protein supplement). The dotted blue box represents the consensus range of tolerance for multivitamin (x-axis) and protein supplement (y-axis), calculated as the values above and below the consensus means that result in an acceptable $Z_{\text {comm }}^{\prime}$ score, $\left|Z_{\text {comm }}^{\prime}\right| \leq 2$. 


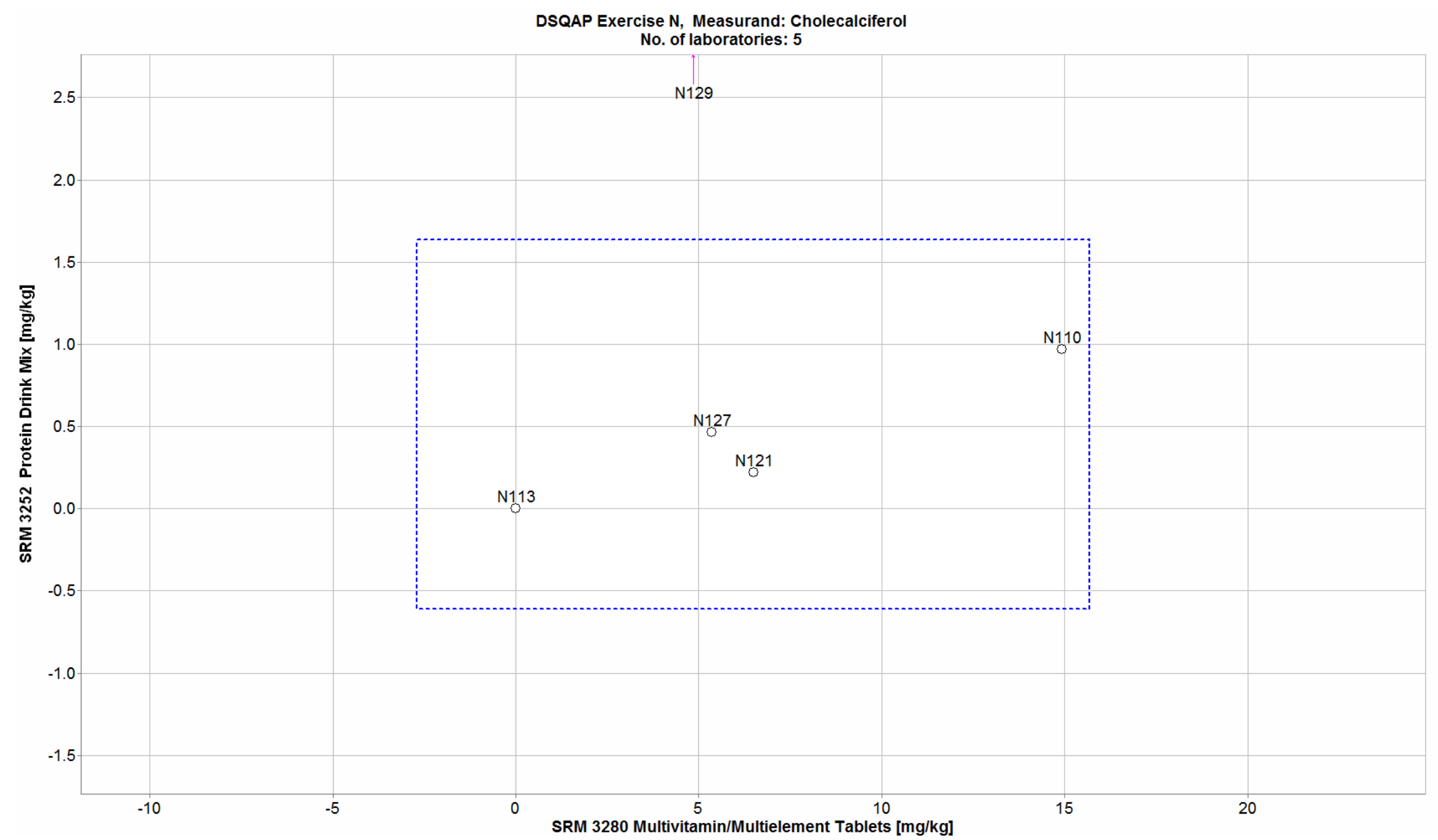

Figure 23. Laboratory means for cholecalciferol (vitamin D3) in SRM 3280 Multivitamin/Multielement Tablets and SRM 3252 Protein Drink Mix (sample/sample comparison view). In this view, the individual laboratory mean for one sample (multivitamin) is compared to the mean for a second sample (protein supplement). The dotted blue box represents the consensus range of tolerance for multivitamin (x-axis) and protein supplement (y-axis), calculated as the values above and below the consensus means that result in an acceptable $Z_{\text {comm }}^{\prime}$ score, $\left|Z_{\text {comm }}^{\prime}\right| \leq 2$. 


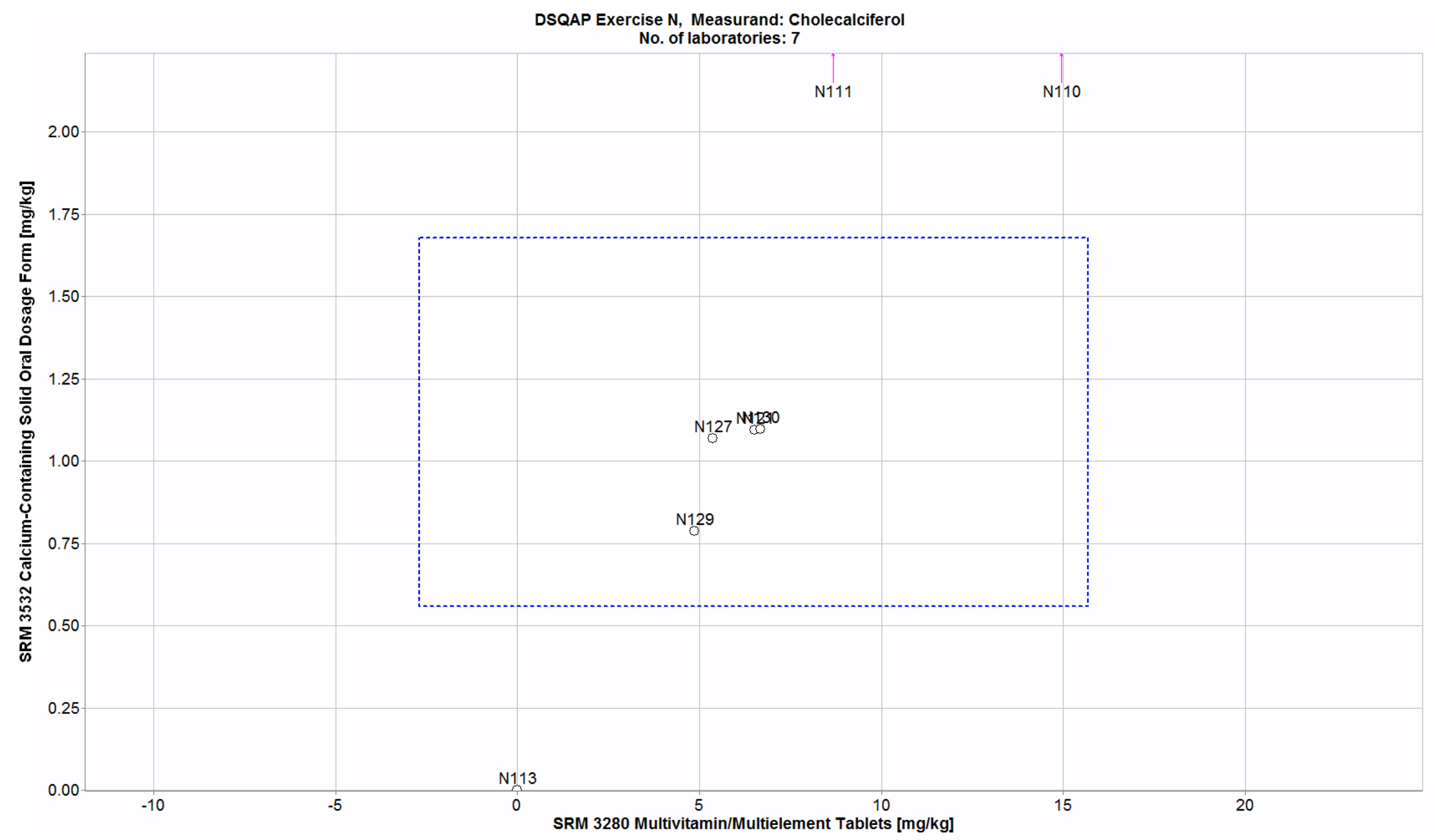

Figure 24. Laboratory means for cholecalciferol (vitamin D3) in SRM 3280 Multivitamin/Multielement Tablets and SRM 3532 Calcium-Containing Solid Oral Dosage Form (sample/sample comparison view). In this view, the individual laboratory mean for one sample (multivitamin) is compared to the mean for a second sample (calcium supplement). The dotted blue box represents the consensus range of tolerance for multivitamin (x-axis) and calcium supplement (y-axis), calculated as the values above and below the consensus means that result in an acceptable $Z_{\text {comm }}^{\prime}$ score, $\left|Z_{\text {comm }}^{\prime}\right| \leq 2$. 


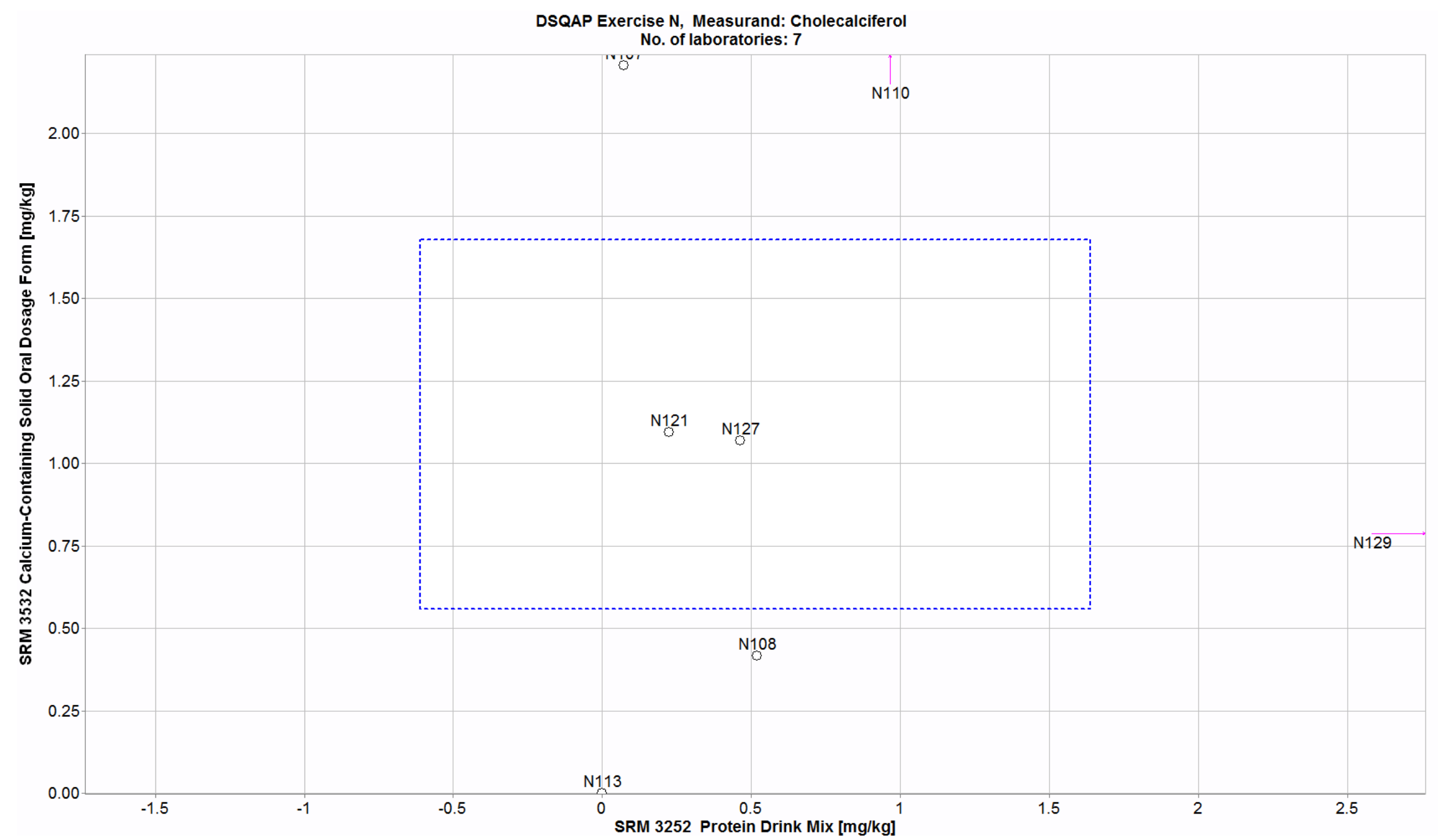

Figure 25. Laboratory means for cholecalciferol (vitamin $D_{3}$ ) in SRM 3252 Protein Drink Mix and SRM 3532 Calcium-Containing Solid Oral Dosage Form (sample/sample comparison view). In this view, the individual laboratory mean for one sample (protein supplement) is compared to the mean for a second sample (calcium supplement). The dotted blue box represents the consensus range of tolerance for protein supplement (x-axis) and calcium supplement (y-axis), calculated as the values above and below the consensus means that result in an acceptable $Z_{\text {comm }}^{\prime}$ score, $\left|Z_{\text {comm }}^{\prime}\right| \leq 2$. 


\section{FATTY ACIDS IN SAW PALMETTO BERRIES AND BORAGE OIL DIETARY SUPPLEMENTS}

\section{Study Overview}

In this study, participants were provided with two NIST SRMs, SRM 3250 Saw Palmetto (Serenoa repens) Fruit, and SRM 3274-1 Borage (Borago officinalis) Oil. Participants were asked to use in-house analytical methods to determine the mass fractions of $\alpha$-linolenic acid, $\gamma$-linolenic acid, linoleic acid, total omega-3 fatty acids, and total omega- 6 fatty acids in each of the matrices and report values in $\mathrm{mg} / \mathrm{g}$ on an as-received basis as fatty acid methyl esters (FAMEs).

\section{$\underline{\text { Sample Information }}$}

Saw Palmetto Berries. Participants were provided with three packets, each containing approximately $6 \mathrm{~g}$ of ground berries. The berries were ground, homogenized, and heat-sealed inside 4 mil polyethylene bags, which were then sealed inside nitrogen-flushed aluminized plastic bags along with two packets of silica gel. Before use, participants were instructed to thoroughly mix the contents of each packet and use a sample size of at least $0.5 \mathrm{~g}$. Participants were asked to store the material at controlled room temperature, $10{ }^{\circ} \mathrm{C}$ to $30{ }^{\circ} \mathrm{C}$, and to report a single value from each packet provided. Approximate analyte levels were not reported to participants prior to the study. The certified values for $\alpha$-linolenic acid and linoleic acid in SRM 3250 Saw Palmetto (Serenoa repens) Fruit were determined at NIST using gas chromatography with flame ionization detection (GC-FID) and GC with mass spectrometric detection (GC-MS). Target values for $\gamma$-linolenic acid, total omega-3 fatty acids, and total omega-6 fatty acids in SRM 3250 have not been determined at NIST. The NIST values and uncertainties for $\alpha$-linolenic acid and linoleic acid are provided in the table below, both on a dry-mass basis as triglycerides, and on an as-received basis as FAMEs ${ }^{5}$, accounting for moisture of the material $(6.42 \%)$.

\section{Certified Mass Fraction in SRM 3250 (mg/g)}

\begin{tabular}{ccccc}
$\underline{\text { Analyte }}$ & (dry-mass basis, as triglyceride) & & (as-received basis, as FAME) \\
\cline { 2 - 3 }$\alpha$-Linolenic Acid & $1.94 \pm 0.25$ & & 1.82 & \pm 0.24 \\
Linoleic Acid & $8.24 \pm 0.55$ & & 7.75 & \pm 0.52
\end{tabular}

Borage Oil. Participants were provided with three ampoules, each containing approximately $1.2 \mathrm{~mL}$ of borage oil. The oil was combined with tert-butylhydroquinone (TBHQ), an antioxidant, and packaged under argon into $2 \mathrm{~mL}$ amber ampoules. Before use, participants were instructed to thoroughly mix the contents of each ampoule and to use a sample size of at least $0.5 \mathrm{~g}$. Participants were asked to store the material under refrigeration, $0{ }^{\circ} \mathrm{C}$ to $4{ }^{\circ} \mathrm{C}$, to prepare a single sample and to report a single value from each ampoule provided. Approximate analyte levels were not reported to participants prior to the study. The certified values for $\gamma$-linolenic acid and linoleic acid, and the reference value for $\alpha$-linolenic acid, in SRM 3274-1 Borage (Borago officinalis) Oil were determined at NIST using GC-FID and GC-MS. Target values for total omega-3 fatty acids and total omega-6 fatty acids in SRM 3274-1 have not been determined at NIST. The NISTdetermined values and standard deviations are reported in the table below on an as-received basis as triglycerides and as FAMEs.

\footnotetext{
${ }^{5}$ De Vries, J.W., Kjos, L., Groff L., Martin, B., Cernohous, K., Patel, H., Payne, M., Leichtweis, H., Shay, M., \& Newcomer, L. (1999) J. AOAC Int. 82, 1146-1155.
} 
NIST-Determined Mass Fraction Values in SRM 3274-1

\begin{tabular}{cccccc} 
Analyte & (mg/g as triglycerides) & & \multicolumn{2}{c}{ (mg/g as FAMEs) } \\
$\alpha$-Linolenic Acid & 1.97 & \pm 0.11 & & 1.98 & \pm \\
$\gamma$-Linolenic Acid & 251 & \pm 24 & 252 & \pm 24 \\
Linoleic Acid & 374 & \pm 35 & 376 & \pm 35
\end{tabular}

$\underline{\text { Study Results }}$

- Thirty laboratories enrolled in the exercise and received samples for some subset of fatty acids in these two samples.

- For the saw palmetto fruit, the greatest participation was for linoleic acid with 18 laboratories (60\% participation). Half of the laboratories reported results for total omega-6 fatty acids, and approximately $40 \%$ of laboratories reported results for $\alpha$-linolenic acid and total omega-3 fatty acids. Only three laboratories reported results for $\gamma$-linolenic acid (10\% participation).

- The consensus mean is below the target range for $\alpha$-linolenic acid and linoleic acid in the saw palmetto fruit.

- The consensus range for $\alpha$-linolenic acid does not overlap the target range.

- The consensus range for linoleic acid slightly overlaps the target range.

- The between laboratory variability ranged from moderate to unacceptable in the saw palmetto fruit for linoleic acid, $\alpha$-linolenic acid, and $\gamma$-linolenic acid $(39 \%, 52 \%$, and $>100 \%$, respectively).

- For the borage oil, the greatest participation was also for linoleic acid with 19 laboratories (63\% participation) and $\gamma$-linolenic acid with 17 laboratories (59\% participation). Fifteen laboratories reported results for total omega- 6 fatty acids (54\% participation), 11 laboratories reported results for $\alpha$-linolenic acid (40\% participation), and 9 laboratories reported results for total omega- 3 fatty acids (32\% participation).

- The consensus mean is within the target range for $\alpha$-linolenic acid in the borage oil.

- The consensus means are below the target range for linoleic acid and $\gamma$-linolenic acid in the borage oil.

- The consensus ranges for linoleic acid, $\alpha$-linolenic acid, and $\gamma$-linolenic acid in the borage oil overlap the target ranges.

- The between-laboratory variability was good for $\alpha$-linolenic acid, $\gamma$-linolenic acid, linoleic acid, and total omega-6 fatty acids in the borage oil (14\% to $29 \%)$.

- The between laboratory variability was unacceptable for total omega-3 fatty acids in the borage oil (>100\%).

- Most laboratories reported using gas chromatography with flame ionization detection (GC-FID) as their analytical method. One or two laboratories reported using GC-MS as the analytical method. 


\section{Technical Recommendations}

The following recommendations are based on results obtained from the participants in this study.

- Results for the saw palmetto berries were highly variable. However, no methods were presented as significantly better or worse than any other and no systematic biases were noted.

- The fatty acids in the saw palmetto berries are challenging to extract and require multiple extraction steps. In past DSQAP exercises, this matrix has proven to be challenging for laboratories, and results are typically below the NIST target ranges.

- While a laboratory should always evaluate the extraction efficiency for completeness, this particular matrix (saw palmetto berries) does not represent a large portion of the market share and should therefore be treated as an unfamiliar matrix and an example for potential bias.

- Results for the borage oil were good overall. No methods were presented as significantly better or worse than any other and no systematic biases were noted.

- For most of the fatty acids, the between-laboratory variability was quite good, likely because the sample did not require significant preparation prior to analysis.

- Wide variability was observed for the total omega-3 fatty acids. The levels of omega-3 fatty acids in borage oil are known to be very low, which likely resulted in the increased variability.

- Note that the two laboratories that reported using GC-MS consistently reported values on the lower end of all laboratories. This trend is not necessarily significant, given that only two laboratories reported using this approach, but worth noting.

- Participants were asked to report concentrations for fatty acids as fatty acid methyl esters (FAMEs). In this case, FAMEs should be used as calibrants or non-esterified fatty acids (e.g., triglycerides) should be carried through the entire sample preparation procedure (hydrolysis and derivatization) to improve quantitation.

- Knowledge of calibrant response when carried through the derivatization procedure is necessary.

- Similarly, for those laboratories using GC-MS, quantitation for some compounds may be inaccurate as a result of non-unity response factors from EI fragmentation. 
Table 12. Individualized data summary table (NIST) for fatty acids in dietary supplements.

\section{National Institute of Standards \& Technology}

\begin{tabular}{|c|c|c|c|c|c|c|c|c|c|c|c|}
\hline \multirow[b]{3}{*}{ Analyte } & \multicolumn{9}{|c|}{ Exercise N - May 2017 - Fatty Acids } & \multirow{2}{*}{\multicolumn{2}{|c|}{ 3. Target }} \\
\hline & \multirow{2}{*}{ Lab Code: } & \multirow{2}{*}{$\begin{array}{l}\text { NIST } \\
\text { Units }\end{array}$} & \multicolumn{4}{|c|}{ 1. Your Results } & \multicolumn{3}{|c|}{ 2. Community Results } & & \\
\hline & & & $\mathrm{x}_{\mathrm{i}}$ & $\mathrm{s}_{\mathrm{i}}$ & $Z_{\text {comm }}^{\prime}$ & $Z_{\text {NIST }}$ & $\mathrm{N}$ & $\mathrm{x}^{*}$ & $\mathrm{~s}^{*}$ & $\mathrm{x}_{\mathrm{NIST}}$ & $U_{95}$ \\
\hline$\alpha$-Linolenic Acid (as FAME) & Saw Palmeto (Serenoa repens) Fruit & $\mathrm{mg} / \mathrm{g}$ & 2.08 & 0.27 & & 0.00 & 12 & 0.67 & 0.37 & 2.08 & 0.27 \\
\hline$\alpha$-Linolenic Acid (as FAME) & Borage (Borago Officinalis) Oil & $\mathrm{mg} / \mathrm{g}$ & 1.98 & 0.09 & & 0.00 & 11 & 2.08 & 0.29 & 1.98 & 0.09 \\
\hline$\gamma$-Linolenic Acid (as FAME) & Saw Palmeto (Serenoa repens) Fruit & $\mathrm{mg} / \mathrm{g}$ & & & & & 3 & 0.36 & 0.60 & & \\
\hline$\gamma$-Linolenic Acid (as FAME) & Borage (Borago Officinalis) Oil & $\mathrm{mg} / \mathrm{g}$ & 252 & 23 & & 0.00 & 17 & 218 & 41 & 252 & 23 \\
\hline Linoleic Acid (as FAME) & Saw Palmeto (Serenoa repens) Fruit & $\mathrm{mg} / \mathrm{g}$ & 8.85 & 0.59 & & 0.00 & 18 & 5.85 & 2.28 & 8.85 & 0.59 \\
\hline Linoleic Acid (as FAME) & Borage (Borago Officinalis) Oil & $\mathrm{mg} / \mathrm{g}$ & 376 & 33 & & 0.00 & 19 & 340 & 63 & 376 & 33 \\
\hline Total Omega-3 Fatty Acid (as FAME) & Saw Palmeto (Serenoa repens) Fruit & $\mathrm{mg} / \mathrm{g}$ & & & & & 10 & 0.83 & 0.59 & & \\
\hline Total Omega-3 Fatty Acid (as FAME) & Borage (Borago Officinalis) Oil & $\mathrm{mg} / \mathrm{g}$ & & & & & 9 & 13 & 19 & & \\
\hline Total Omega-6 Fatty Acid (as FAME) & Saw Palmeto (Serenoa repens) Fruit & $\mathrm{mg} / \mathrm{g}$ & & & & & 14 & 5.48 & 1.73 & & \\
\hline Total Omega-6 Fatty Acid (as FAME) & Borage (Borago Officinalis) Oil & $\mathrm{mg} / \mathrm{g}$ & & & & & 15 & 550 & 161 & & \\
\hline
\end{tabular}

$\mathrm{x}_{\mathrm{i}}$ Mean of reported values

$\mathrm{s}_{\mathrm{i}}$ Standard deviation of reported values

$Z_{\text {comm }} Z^{\prime}$-score with respect to community consensus

$\mathrm{Z}_{\text {NIST }} \mathrm{Z}$-score with respect to NIST value
$\mathrm{N}$ Number of quantitative values reported

$x^{*}$ Robust mean of reported values

$\mathrm{s} *$ Robust standard deviation
$\mathrm{x}_{\mathrm{NIST}}$ NIST-assessed value

$U_{95} \pm 95 \%$ confidence interval about the assessed value or standard deviation $\left(\mathrm{s}_{\mathrm{NIST}}\right)$ 
Table 13. Data summary table for $\alpha$-linolenic acid (as FAME) in dietary supplements. Data highlighted in red have been flagged as potential outliers (e.g., difference from reference value, Grubb and/or Cochran) by the NIST software package.

\begin{tabular}{|c|c|c|c|c|c|c|c|c|c|c|c|}
\hline & \multirow[b]{3}{*}{ Lab } & \multicolumn{10}{|c|}{$\alpha$-Linolenic Acid (as FAME) } \\
\hline & & \multicolumn{5}{|c|}{$\begin{array}{l}\alpha \text {-Linolenic A } \\
\text { SRM } 3250 \text { Saw Palmetto (Serenoa repens) Fruit }(\mathrm{mg} / \mathrm{g})\end{array}$} & \multicolumn{5}{|c|}{ SRM 3274-1 Borage (Borago officinalis) Oil (mg/g) } \\
\hline & & $\mathbf{A}$ & B & $\mathbf{C}$ & Avg & SD & $\mathbf{A}$ & B & $\mathbf{C}$ & Avg & SD \\
\hline \multirow{30}{*}{ 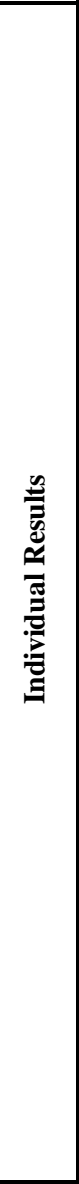 } & NIST & & & & 1.824 & 0.235 & & & & 1.98 & 0.09 \\
\hline & N102 & & & & & & 1.93 & & & 1.93 & \\
\hline & N103 & & & & & & & & & & \\
\hline & N105 & & & & & & & & & & \\
\hline & N107 & 1.055 & 0.875 & 0.840 & 0.923 & 0.115 & 2.02 & 1.52 & 1.92 & 1.82 & 0.27 \\
\hline & N108 & 0.700 & 0.710 & 0.700 & 0.703 & 0.006 & 2.05 & 2.05 & 2.05 & 2.05 & 0.00 \\
\hline & N109 & 0.356 & 0.407 & 0.365 & 0.376 & 0.027 & & & & & \\
\hline & N110 & 23.700 & 23.600 & 23.900 & 23.733 & 0.153 & 77.30 & 76.10 & 76.00 & 76.47 & 0.72 \\
\hline & N112 & 0.700 & 0.700 & 0.600 & 0.667 & 0.058 & 2.00 & 2.00 & 2.10 & 2.03 & 0.06 \\
\hline & N113 & & & & & & & & & & \\
\hline & N115 & & & & & & & & & & \\
\hline & N118 & 0.649 & 0.676 & 0.583 & 0.636 & 0.048 & 1.79 & 1.80 & 1.88 & 1.83 & 0.05 \\
\hline & N120 & & & & & & & & & & \\
\hline & N121 & 0.466 & 0.497 & 0.511 & 0.491 & 0.023 & 2.30 & 2.27 & 2.29 & 2.29 & 0.02 \\
\hline & N123 & & & & & & & & & & \\
\hline & N124 & 2.070 & 1.570 & 1.200 & 1.613 & 0.437 & 2.06 & 2.08 & 2.06 & 2.07 & 0.01 \\
\hline & N125 & & & & & & & & & & \\
\hline & N126 & & & & & & & & & & \\
\hline & N129 & & & & & & & & & & \\
\hline & N130 & & & & & & & & & & \\
\hline & N132 & 0.550 & 0.540 & 0.530 & 0.540 & 0.010 & 1.99 & 1.99 & 2.02 & 2.00 & 0.02 \\
\hline & N133 & 0.727 & 0.740 & 0.784 & 0.750 & 0.030 & 2.50 & 2.71 & 2.33 & 2.51 & 0.19 \\
\hline & N136 & 0.260 & 0.230 & 0.340 & 0.277 & 0.057 & & & & & \\
\hline & N137 & 0.200 & 0.200 & 0.300 & 0.233 & 0.058 & $<0.000$ & $<0.000$ & $<0.000$ & $<0.000$ & \\
\hline & N141 & & & & & & & & & & \\
\hline & N147 & & & & & & & & & & \\
\hline & N150 & & & & & & & & & & \\
\hline & N155 & & & & & & & & & & \\
\hline & N157 & $<0.000$ & $<0.000$ & $<0.000$ & $<0.000$ & & $<0.000$ & $<0.000$ & $<0.000$ & $<0.000$ & \\
\hline & N158 & & & & & & 1.80 & 1.80 & 1.80 & 1.80 & 0.00 \\
\hline \multirow{5}{*}{ 象 } & & \multirow{5}{*}{\multicolumn{3}{|c|}{$\begin{array}{l}\text { Consensus Mean } \\
\text { Consensus Standard Deviation } \\
\text { Maximum } \\
\text { Minimum } \\
\mathrm{N}\end{array}$}} & 0.671 & & \multirow{5}{*}{\multicolumn{3}{|c|}{$\begin{array}{l}\text { Consensus Mean } \\
\text { Consensus Standard Deviation } \\
\text { Maximum } \\
\text { Minimum } \\
\mathrm{N}\end{array}$}} & 2.08 & \\
\hline & & & & & 0.372 & & & & & 0.29 & \\
\hline & & & & & 23.733 & & & & & 76.47 & \\
\hline & & & & & 0.233 & & & & & 1.80 & \\
\hline & & & & & 12 & & & & & 11 & \\
\hline
\end{tabular}


Table 14. Data summary table for $\gamma$-linolenic (as FAME) acid in dietary supplements. Data highlighted in red have been flagged as potential outliers (e.g., difference from reference value, Grubb and/or Cochran) by the NIST software package.

\begin{tabular}{|c|c|c|c|c|c|c|c|c|c|c|c|}
\hline & \multirow[b]{3}{*}{ Lab } & \multicolumn{10}{|c|}{$\gamma$-Linolenic Acid (as FAME) } \\
\hline & & \multicolumn{5}{|c|}{$\begin{array}{l}\gamma \text {-Linolenic A } \\
\text { SRM } 3250 \text { Saw Palmetto (Serenoa repens ) Fruit }(\mathrm{mg} / \mathrm{g})\end{array}$} & \multicolumn{5}{|c|}{ SRM 3274-1 Borage (Borago officinalis) Oil (mg/g) } \\
\hline & & $\mathbf{A}$ & $\mathbf{B}$ & $\mathbf{C}$ & Avg & SD & $\mathbf{A}$ & B & $\mathbf{C}$ & Avg & SD \\
\hline \multirow{30}{*}{ 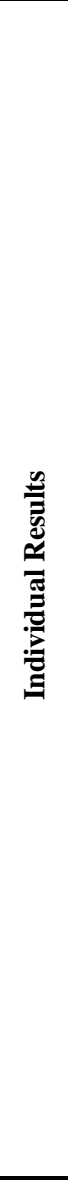 } & NIST & & & & & & & & & 252 & 23 \\
\hline & N102 & & & & & & 233 & & & 233 & \\
\hline & N103 & & & & & & & & & & \\
\hline & N105 & 0.950 & 0.970 & 0.990 & 0.970 & 0.020 & 2.01 & 2.60 & 1.70 & 2.10 & 0.46 \\
\hline & N107 & & & & & & 260 & 247 & 254 & 254 & 6 \\
\hline & N108 & & & & & & 242 & 241 & 241 & 241 & 1 \\
\hline & N109 & & & & & & & & & & \\
\hline & N110 & & & & & & 128 & 127 & 124 & 126 & 2 \\
\hline & N112 & $<0.100$ & $<0.100$ & $<0.100$ & $<0.100$ & & 226 & 226 & 226 & 226 & 0 \\
\hline & N113 & & & & & & & & & & \\
\hline & N115 & & & & & & & & & & \\
\hline & N118 & & & & & & 226 & 232 & 225 & 228 & 4 \\
\hline & N120 & & & & & & & & & & \\
\hline & N121 & & & & & & 234 & 235 & 232 & 234 & 2 \\
\hline & N123 & & & & & & 149 & 143 & 147 & 146 & 3 \\
\hline & N124 & 0.030 & 0.020 & 0.010 & 0.020 & 0.010 & 237 & 239 & 237 & 237 & 1 \\
\hline & N125 & & & & & & & & & & \\
\hline & N126 & & & & & & & & & & \\
\hline & N129 & & & & & & & & & & \\
\hline & N130 & & & & & & 243 & 240 & 241 & 241 & 1 \\
\hline & N132 & & & & & & 206 & 203 & 207 & 205 & 2 \\
\hline & N133 & & & & & & 270 & 275 & 263 & 269 & 6 \\
\hline & N136 & 0.020 & 0.020 & 0.210 & 0.083 & 0.110 & 168 & 171 & 171 & 170 & 2 \\
\hline & N137 & $<0.000$ & $<0.000$ & $<0.000$ & $<0.000$ & & 237 & 238 & 237 & 237 & 1 \\
\hline & N141 & & & & & & & & & & \\
\hline & N147 & & & & & & & & & & \\
\hline & N150 & & & & & & & & & & \\
\hline & N155 & & & & & & & & & & \\
\hline & N157 & $<0.000$ & $<0.000$ & $<0.000$ & $<0.000$ & & 215 & 221 & 214 & 217 & 4 \\
\hline & N158 & & & & & & 239 & 240 & 240 & 240 & 1 \\
\hline \multirow{5}{*}{ 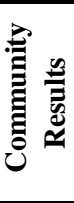 } & & \multirow{5}{*}{\multicolumn{3}{|c|}{$\begin{array}{l}\text { Consensus Standard Deviation } \\
\text { Maximum } \\
\text { Minimum } \\
\mathrm{N}\end{array}$}} & 0.358 & & \multirow{5}{*}{\multicolumn{3}{|c|}{$\begin{array}{l}\text { Consensus } 1 \\
\text { Consensus } S \\
\text { Maximum } \\
\text { Minimum } \\
\mathrm{N}\end{array}$}} & 218 & \\
\hline & & & & & 0.603 & & & & & 41 & \\
\hline & & & & & 0.970 & & & & & 269 & \\
\hline & & & & & 0.020 & & & & & 2.10 & \\
\hline & & & & & 3 & & & & & 17 & \\
\hline
\end{tabular}


Table 15. Data summary table for linoleic acid (as FAME) in dietary supplements. Data highlighted in red have been flagged as potential outliers (e.g., difference from reference value, Grubb and/or Cochran) by the NIST software package.

\begin{tabular}{|c|c|c|c|c|c|c|c|c|c|c|c|}
\hline & \multirow[b]{3}{*}{ Lab } & \multicolumn{10}{|c|}{ Linoleic Acid (as FAME) } \\
\hline & & \multicolumn{5}{|c|}{ SRM 3250 Saw Palmetto (Serenoa repens) Fruit $(\mathrm{mg} / \mathrm{g})$} & \multicolumn{5}{|c|}{ SRM 3274-1 Borage (Borago officinalis) Oil (mg/g) } \\
\hline & & $\mathbf{A}$ & B & $\mathbf{C}$ & Avg & SD & $\mathbf{A}$ & B & $\mathbf{C}$ & Avg & SD \\
\hline \multirow{31}{*}{ 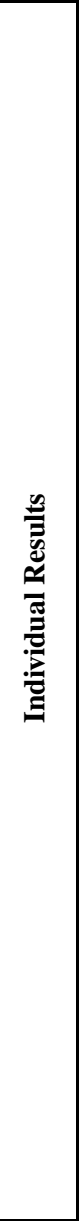 } & NIST & & & & 7.75 & 0.52 & & & & 376 & 33 \\
\hline & N102 & 1.11 & & & 1.11 & & 0.04 & & & 0.04 & \\
\hline & N103 & & & & & & & & & & \\
\hline & N105 & 6.56 & 6.96 & 6.76 & 6.76 & 0.20 & 281 & 283 & 276 & 280 & 4 \\
\hline & N107 & 6.27 & 6.24 & 6.29 & 6.26 & 0.02 & 427 & 421 & 401 & 416 & 14 \\
\hline & N108 & 5.80 & 5.84 & 5.86 & 5.83 & 0.03 & 371 & 370 & 370 & 370 & 1 \\
\hline & N109 & 3.60 & 3.72 & 3.41 & 3.58 & 0.15 & & & & & \\
\hline & N110 & 23.70 & 23.60 & 23.90 & 23.73 & 0.15 & 206 & 203 & 200 & 203 & 3 \\
\hline & N112 & 5.80 & 6.20 & 5.70 & 5.90 & 0.26 & 353 & 352 & 352 & 352 & 1 \\
\hline & N113 & & & & & & & & & & \\
\hline & N115 & & & & & & & & & & \\
\hline & N117 & 6.98 & 6.95 & 7.03 & 6.99 & 0.04 & 427 & 425 & 428 & 427 & 2 \\
\hline & N118 & 5.64 & 5.76 & 5.41 & 5.60 & 0.18 & 370 & 388 & 371 & 376 & 10 \\
\hline & N120 & & & & & & & & & & \\
\hline & N121 & 3.62 & 3.60 & 3.85 & 3.69 & 0.14 & 366 & 367 & 363 & 365 & 2 \\
\hline & N123 & 5.85 & 5.97 & 5.92 & 5.91 & 0.06 & 269 & 258 & 264 & 264 & 6 \\
\hline & N124 & 18.20 & 13.29 & 10.60 & 14.03 & 3.85 & 369 & 373 & 369 & 370 & 2 \\
\hline & N125 & & & & & & & & & & \\
\hline & N126 & & & & & & & & & & \\
\hline & N129 & 11.37 & 11.71 & 11.49 & 11.52 & 0.17 & 351 & 356 & 348 & 351 & 4 \\
\hline & N130 & & & & & & 371 & 369 & 370 & 370 & 1 \\
\hline & N132 & 4.79 & 4.73 & 4.74 & 4.75 & 0.03 & 313 & 309 & 314 & 312 & 3 \\
\hline & N133 & 5.82 & 5.71 & 6.10 & 5.88 & 0.20 & 403 & 410 & 392 & 402 & 9 \\
\hline & N136 & 3.97 & 3.94 & 4.99 & 4.30 & 0.60 & 265 & 275 & 287 & 275 & 11 \\
\hline & N137 & 3.20 & 3.40 & 3.60 & 3.40 & 0.20 & 359 & 357 & 360 & 359 & 2 \\
\hline & N141 & & & & & & & & & & \\
\hline & N147 & & & & & & & & & & \\
\hline & N150 & & & & & & & & & & \\
\hline & N155 & & & & & & & & & & \\
\hline & N157 & 6.15 & 6.18 & 6.23 & 6.19 & 0.04 & 311 & 320 & 308 & 313 & 6 \\
\hline & N158 & & & & & & 364 & 365 & 366 & 365 & 1 \\
\hline \multirow{5}{*}{ 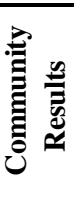 } & & \multirow{5}{*}{\multicolumn{3}{|c|}{$\begin{array}{l}\text { Consensus Mean } \\
\text { Consensus Standa } \\
\text { Maximum } \\
\text { Minimum } \\
\mathrm{N}\end{array}$}} & 5.85 & & \multirow{5}{*}{\multicolumn{3}{|c|}{$\begin{array}{l}\text { Consensus } \\
\text { Consensus } \\
\text { Maximum } \\
\text { Minimum } \\
\mathrm{N}\end{array}$}} & 340 & \\
\hline & & & & & 2.28 & & & & & 63 & \\
\hline & & & & & 23.73 & & & & & 427 & \\
\hline & & & & & 1.11 & & & & & 0.04 & \\
\hline & & & & & 18 & & & & & 19 & \\
\hline
\end{tabular}


Table 16. Data summary table for total omega-3 fatty acids (as FAMEs) in dietary supplements. Data highlighted in red have been flagged as potential outliers (e.g., difference from reference value, Grubb and/or Cochran) by the NIST software package.

\begin{tabular}{|c|c|c|c|c|c|c|c|c|c|c|c|}
\hline & & \multicolumn{10}{|c|}{ Total Omega-3 Fatty Acids (as FAMEs) } \\
\hline & & \multicolumn{5}{|c|}{ SRM 3250 Saw Palmetto (Serenoa repens) Fruit $(\mathrm{mg} / \mathrm{g}$ ) } & \multicolumn{5}{|c|}{ SRM 3274-1 Borage (Borago officinalis) Oil (mg/g) } \\
\hline & Lab & $\mathbf{A}$ & B & $\mathrm{C}$ & Avg & SD & $\mathbf{A}$ & B & $\mathbf{C}$ & Avg & SD \\
\hline \multirow{29}{*}{ 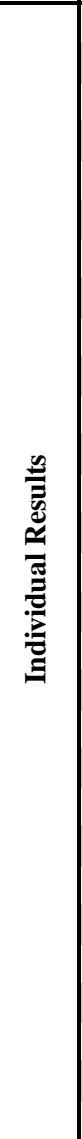 } & NIST & & & & & & & & & & \\
\hline & N103 & & & & & & & & & & \\
\hline & N105 & & & & & & & & & & \\
\hline & N107 & 1.06 & 0.87 & 0.84 & 0.92 & 0.12 & 17.3 & 15.5 & 18.2 & 17.0 & 1.3 \\
\hline & N108 & 0.70 & 0.71 & 0.70 & 0.70 & 0.01 & 2.1 & 2.1 & 2.1 & 2.1 & 0.0 \\
\hline & N109 & & & & & & & & & & \\
\hline & N110 & 23.70 & 23.60 & 23.90 & 23.73 & 0.15 & 77.3 & 76.1 & 76.0 & 76.5 & 0.7 \\
\hline & N112 & 0.70 & 0.80 & 0.70 & 0.73 & 0.06 & 4.0 & 4.2 & 4.2 & 4.1 & 0.1 \\
\hline & N113 & & & & & & & & & & \\
\hline & N115 & & & & & & & & & & \\
\hline & N118 & & & & & & & & & & \\
\hline & N120 & & & & & & & & & & \\
\hline & N121 & 0.50 & 0.51 & 0.54 & 0.52 & 0.02 & 78.6 & 79.9 & 78.2 & 78.9 & 0.9 \\
\hline & N123 & & & & & & & & & & \\
\hline & N124 & 18.23 & 13.31 & 10.61 & 14.05 & 3.86 & 2.1 & 2.1 & 2.1 & 2.1 & 0.0 \\
\hline & N125 & & & & & & & & & & \\
\hline & N126 & & & & & & & & & & \\
\hline & N129 & & & & & & & & & & \\
\hline & N130 & & & & & & & & & & \\
\hline & N132 & 0.59 & 0.57 & 0.56 & 0.57 & 0.02 & 2.1 & 2.1 & 2.1 & 2.1 & 0.0 \\
\hline & N133 & 0.93 & 0.86 & 0.92 & 0.90 & 0.04 & 2.9 & 2.9 & 2.6 & 2.8 & 0.2 \\
\hline & N136 & 0.26 & 0.23 & 0.34 & 0.28 & 0.06 & & & & & \\
\hline & N137 & 0.20 & 0.20 & 0.30 & 0.23 & 0.06 & $<0.000$ & $<0.000$ & $<0.000$ & $<0.000$ & \\
\hline & N141 & & & & & & & & & & \\
\hline & N147 & & & & & & & & & & \\
\hline & N150 & & & & & & & & & & \\
\hline & N155 & & & & & & & & & & \\
\hline & N157 & $<0.000$ & $<0.000$ & $<0.000$ & $<0.000$ & & $<0.000$ & $<0.000$ & $<0.000$ & $<0.000$ & \\
\hline & N158 & & & & & & 3.6 & 3.7 & 3.6 & 3.6 & 0.1 \\
\hline \multirow{5}{*}{ 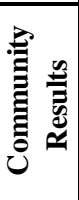 } & & \multirow{5}{*}{\multicolumn{3}{|c|}{$\begin{array}{l}\text { Consensus Mean } \\
\text { Consensus Standard Deviation } \\
\text { Maximum } \\
\text { Minimum } \\
\mathrm{N}\end{array}$}} & 0.83 & & \multirow{5}{*}{\multicolumn{3}{|c|}{$\begin{array}{l}\text { Consensus Mean } \\
\text { Consensus Standard Deviation } \\
\text { Maximum } \\
\text { Minimum } \\
\mathrm{N}\end{array}$}} & 12.9 & \\
\hline & & & & & 0.59 & & & & & 18.9 & \\
\hline & & & & & 23.73 & & & & & 78.9 & \\
\hline & & & & & 0.23 & & & & & 2.1 & \\
\hline & & & & & 10 & & & & & 9 & \\
\hline
\end{tabular}


Table 17. Data summary table for total omega-6 fatty acids (as FAMEs) in dietary supplements. Data highlighted in red have been flagged as potential outliers (e.g., difference from reference value, Grubb and/or Cochran) by the NIST software package.

\begin{tabular}{|c|c|c|c|c|c|c|c|c|c|c|c|}
\hline & & \multicolumn{10}{|c|}{ Total Omega-6 Fatty Acids (as FAMEs) } \\
\hline & & \multicolumn{5}{|c|}{ SRM 3250 Saw Palmetto (Serenoa repens) Fruit (mg/g) } & \multicolumn{5}{|c|}{ SRM 3274-1 Borage (Borago officinalis) Oil $(\mathrm{mg} / \mathrm{g})$} \\
\hline & Lab & $\mathbf{A}$ & B & $\mathbf{C}$ & Avg & SD & $\mathbf{A}$ & B & $\mathbf{C}$ & Avg & SD \\
\hline \multirow{29}{*}{ 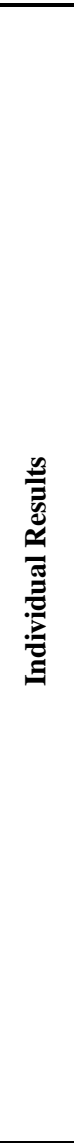 } & NIST & & & & & & & & & & \\
\hline & N103 & & & & & & & & & & \\
\hline & N105 & 84.20 & 94.10 & 88.50 & 88.93 & 4.96 & 985 & 990 & 969 & 981 & 11 \\
\hline & N107 & 6.27 & 6.24 & 6.29 & 6.27 & 0.03 & 688 & 670 & 657 & 672 & 16 \\
\hline & N108 & 5.80 & 5.84 & 5.86 & 5.83 & 0.03 & 612 & 611 & 610 & 611 & 1 \\
\hline & N109 & & & & & & & & & & \\
\hline & N110 & 4.66 & 4.58 & 4.62 & 4.62 & 0.04 & 325 & 321 & 316 & 321 & 5 \\
\hline & N112 & 5.80 & 6.30 & 5.80 & 5.97 & 0.29 & 582 & 581 & 580 & 581 & 1 \\
\hline & N113 & & & & & & & & & & \\
\hline & N115 & & & & & & & & & & \\
\hline & N118 & & & & & & & & & & \\
\hline & N120 & & & & & & & & & & \\
\hline & N121 & 3.62 & 3.60 & 3.85 & 3.69 & 0.14 & 600 & 602 & 595 & 599 & 4 \\
\hline & N123 & 5.85 & 5.97 & 5.92 & 5.91 & 0.06 & 436 & 418 & 428 & 427 & 9 \\
\hline & N124 & 2.07 & 1.57 & 1.20 & 1.61 & 0.44 & 606 & 612 & 606 & 608 & 3 \\
\hline & N125 & & & & & & & & & & \\
\hline & N126 & & & & & & & & & & \\
\hline & N129 & 11.37 & 11.71 & 11.49 & 11.52 & 0.17 & 351 & 356 & 348 & 351 & 4 \\
\hline & N130 & & & & & & & & & & \\
\hline & N132 & 4.88 & 4.80 & 4.81 & 4.83 & 0.04 & 521 & 515 & 524 & 520 & 5 \\
\hline & N133 & 5.98 & 5.87 & 6.26 & 6.04 & 0.20 & 705 & 716 & 685 & 702 & 16 \\
\hline & N136 & 4.23 & 4.17 & 5.32 & 4.57 & 0.65 & 265 & 275 & 287 & 275 & 11 \\
\hline & N137 & 3.60 & 3.80 & 4.00 & 3.80 & 0.20 & 624 & 623 & 625 & 624 & 1 \\
\hline & N141 & & & & & & & & & & \\
\hline & N147 & & & & & & & & & & \\
\hline & N150 & & & & & & & & & & \\
\hline & N155 & & & & & & & & & & \\
\hline & N157 & 6.15 & 6.18 & 6.23 & 6.19 & 0.04 & 526 & 541 & 522 & 530 & 10 \\
\hline & N158 & & & & & & 605 & 608 & 609 & 607 & 2 \\
\hline \multirow{5}{*}{ 气 } & & \multicolumn{3}{|c|}{ Consensus Mean } & \multicolumn{2}{|l|}{5.48} & \multicolumn{3}{|c|}{ Consensus Mean } & 550 & \\
\hline & & \multicolumn{3}{|c|}{ Consensus Standard Deviation } & \multicolumn{2}{|l|}{1.73} & \multicolumn{3}{|c|}{ Consensus Standard Deviation } & 161 & \\
\hline & & \multicolumn{3}{|c|}{ Maximum } & \multicolumn{2}{|l|}{88.93} & \multicolumn{3}{|c|}{ Maximum } & 981 & \\
\hline & & \multicolumn{3}{|c|}{ Minimum } & \multicolumn{2}{|l|}{1.61} & \multicolumn{3}{|c|}{ Minimum } & 275 & \\
\hline & & \multicolumn{3}{|l|}{$\mathrm{N}$} & & \multicolumn{3}{|c|}{$\mathrm{N}$} & 15 & \\
\hline
\end{tabular}




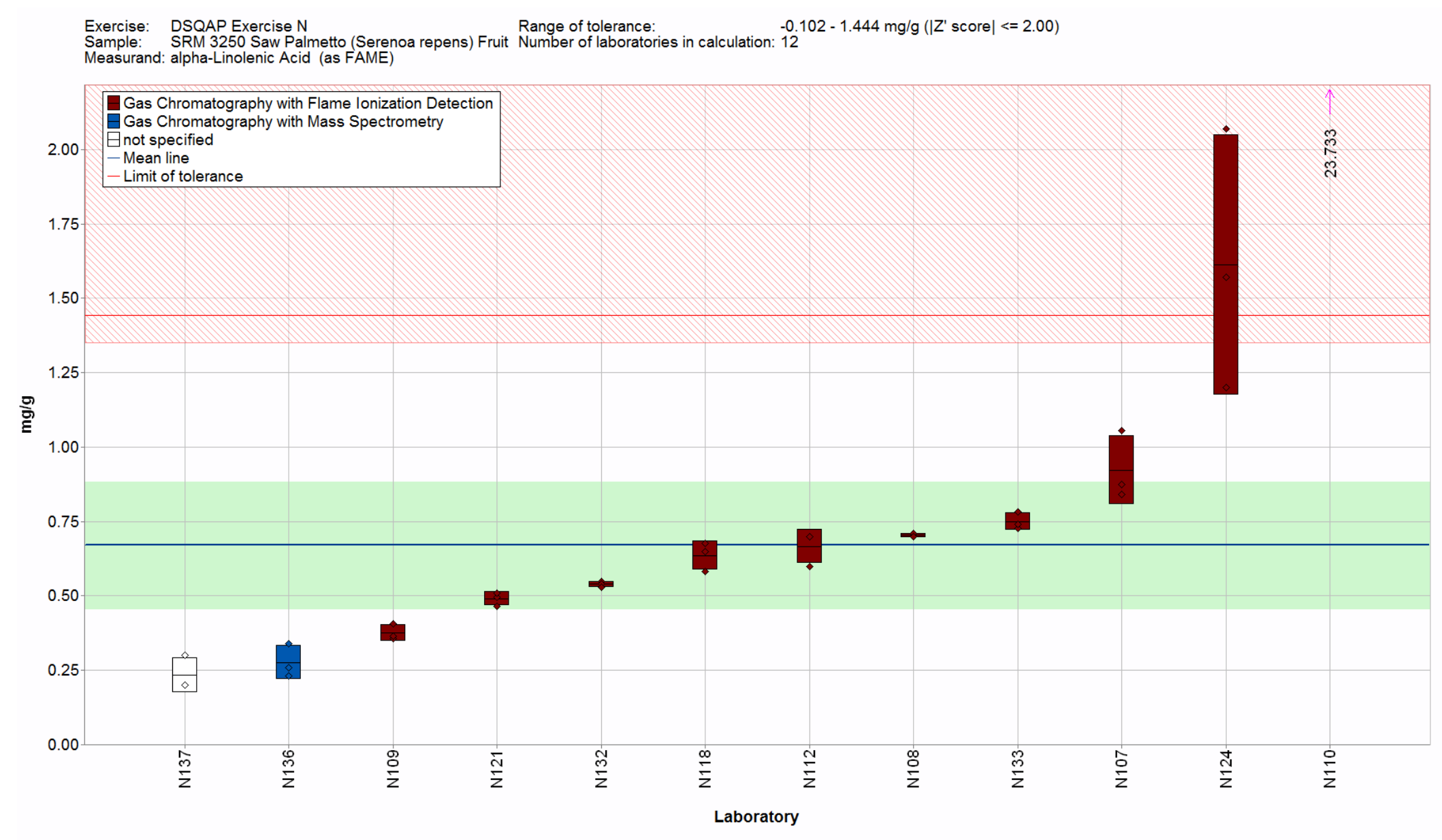

Figure 26. $\alpha$-Linolenic acid (as FAME) in SRM 3250 Saw Palmetto (Serenoa repens) Fruit (data summary view - analytical method). In this view, individual laboratory data are plotted (diamonds) with the individual laboratory standard deviation (rectangle). The color of the data point represents the analytical method employed. The solid blue line represents the consensus mean and the green shaded region represents the $95 \%$ confidence interval for the consensus mean. The solid red lines represent the upper consensus range of tolerance, calculated as the values above and below the consensus mean that result in an acceptable $Z_{\text {comm }}^{\prime}$ score, $\left|Z_{\text {comm }}^{\prime}\right| \leq 2$, with the lower range set a zero. The red shaded region represents the NIST range of tolerance, which encompasses the NIST certified value bounded by twice its uncertainty $\left(U_{95}\right)$ and represents the range that results in an acceptable $Z_{\text {NIST }}$ score, $\left|Z_{\text {NIST }}\right| \leq 2$. 


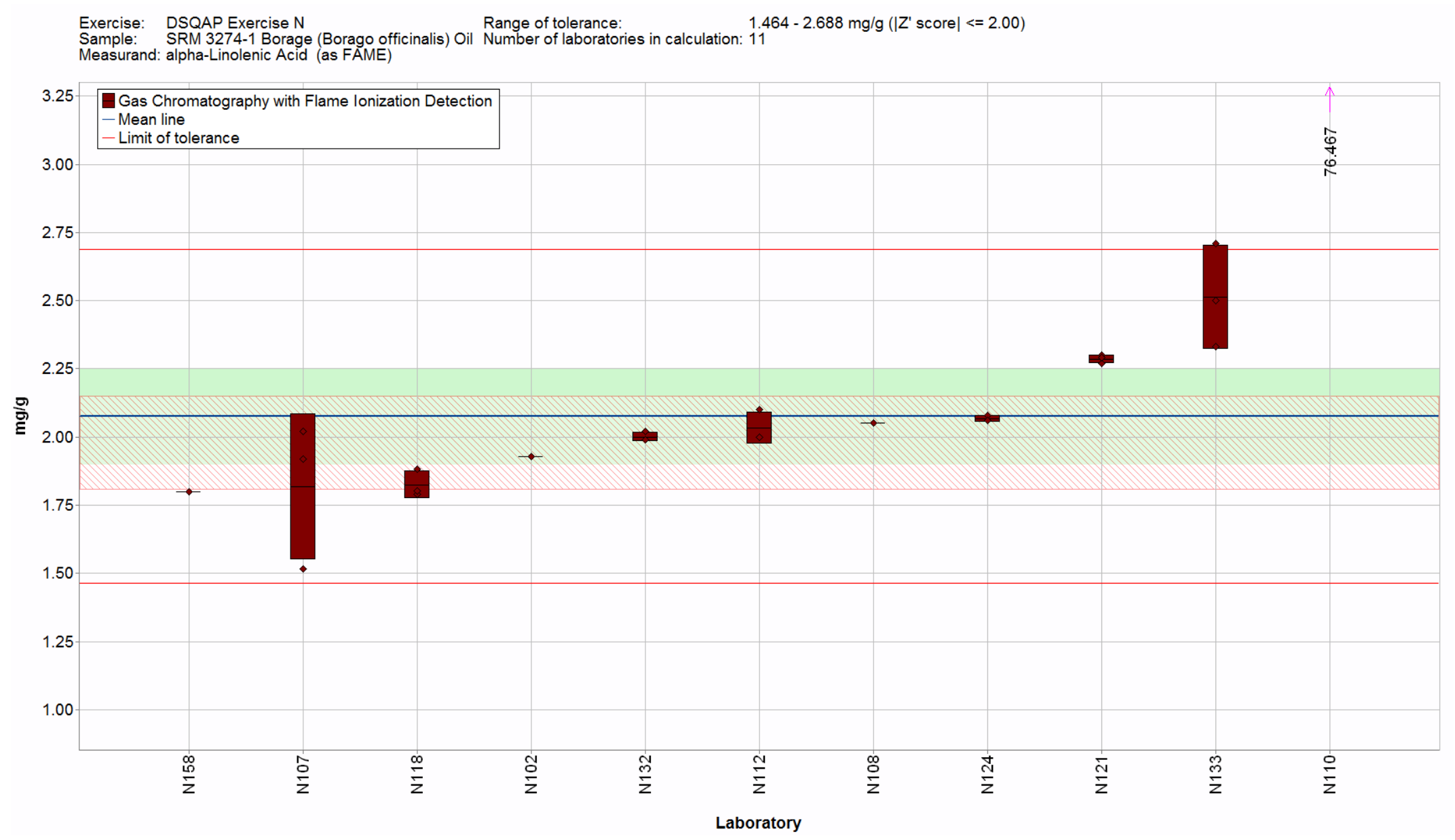

Figure 27. $\alpha$-Linolenic acid (as FAME) in SRM 3274-1 Borage (Borago officinalis) Oil (data summary view - analytical method). In this view, individual laboratory data are plotted (diamonds) with the individual laboratory standard deviation (rectangle). The color of the data point represents the analytical method employed. The solid blue line represents the consensus mean and the green shaded region represents the $95 \%$ confidence interval for the consensus mean. The solid red lines represent the consensus range of tolerance, calculated as the values above and below the consensus mean that result in an acceptable $Z_{\text {comm }}^{\prime}$ score, $\left|Z_{\text {comm }}^{\prime}\right| \leq 2$. The red shaded region represents the NIST range of tolerance, which encompasses the NIST reference value bounded by twice its uncertainty ( $\left.U_{95}\right)$ and represents the range that results in an acceptable $Z_{\text {NIST }}$ score, $\left|Z_{\text {NIST }}\right| \leq 2$. 


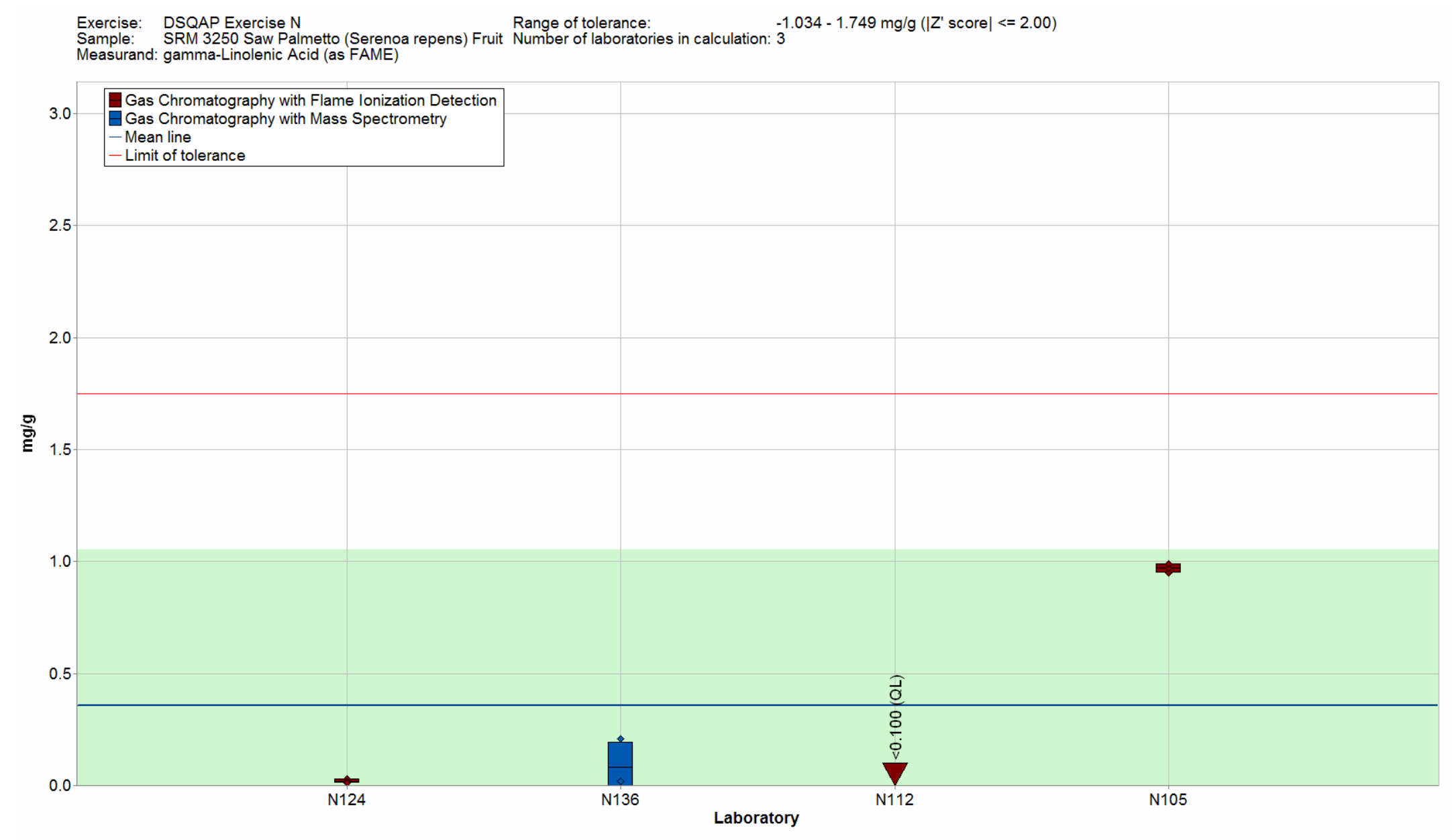

Figure 28. $\gamma$-Linolenic acid (as FAME) in SRM 3250 Saw Palmetto (Serenoa repens) Fruit (data summary view - analytical method). In this view, individual laboratory data are plotted (diamonds) with the individual laboratory standard deviation (rectangle). The color of the data point represents the analytical method employed. Laboratory data shown as a triangle indicates that a "less than" result was submitted, and the base of the triangle is displayed at the reported laboratory detection limit. The solid blue line represents the consensus mean and the green shaded region represents the $95 \%$ confidence interval for the consensus mean. The solid red line represents the upper consensus range of tolerance, calculated as the values above and below the consensus mean that result in an acceptable $Z_{\text {comm }}^{\prime}$ score, $\left|Z_{\text {comm }}^{\prime}\right| \leq 2$, with the lower range set at zero. A NIST value has not been determined in this material. 


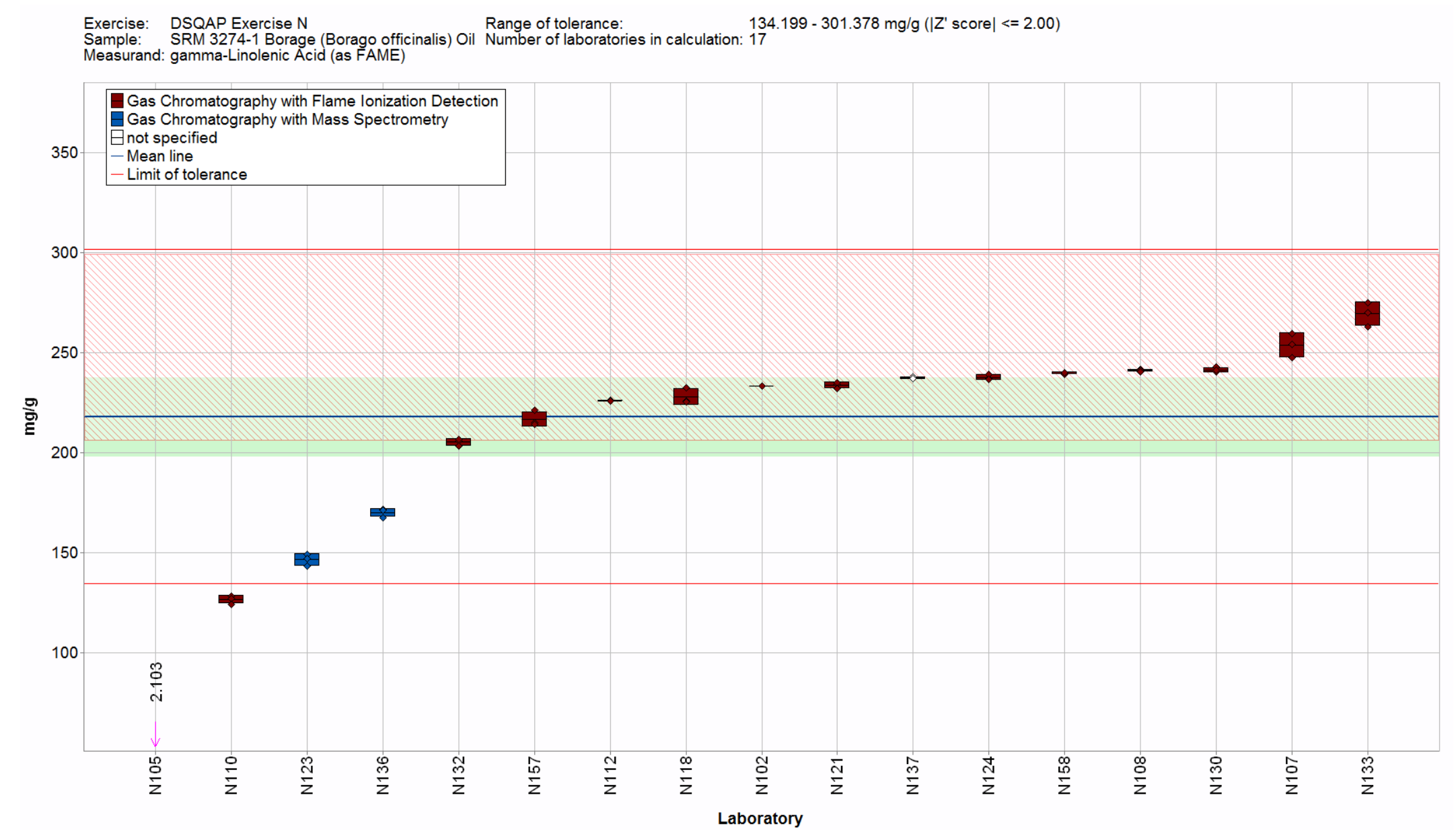

Figure 29. $\gamma$-Linolenic acid (as FAME) in SRM 3274-1 Borage (Borago officinalis) Oil (data summary view - analytical method). In this view, individual laboratory data are plotted (diamonds) with the individual laboratory standard deviation (rectangle). The color of the data point represents the analytical method employed. The solid blue line represents the consensus mean and the green shaded region represents the $95 \%$ confidence interval for the consensus mean. The solid red lines represent the consensus range of tolerance, calculated as the values above and below the consensus mean that result in an acceptable $Z_{\text {comm }}^{\prime}$ score, $\left|Z_{\text {comm }}^{\prime}\right| \leq 2$. The red shaded region represents the NIST range of tolerance, which encompasses the NIST certified value bounded by twice its uncertainty ( $\left.U_{95}\right)$ and represents the range that results in an acceptable $Z_{\text {NIST }}$ score, $\left|Z_{\text {NIST }}\right| \leq 2$. 


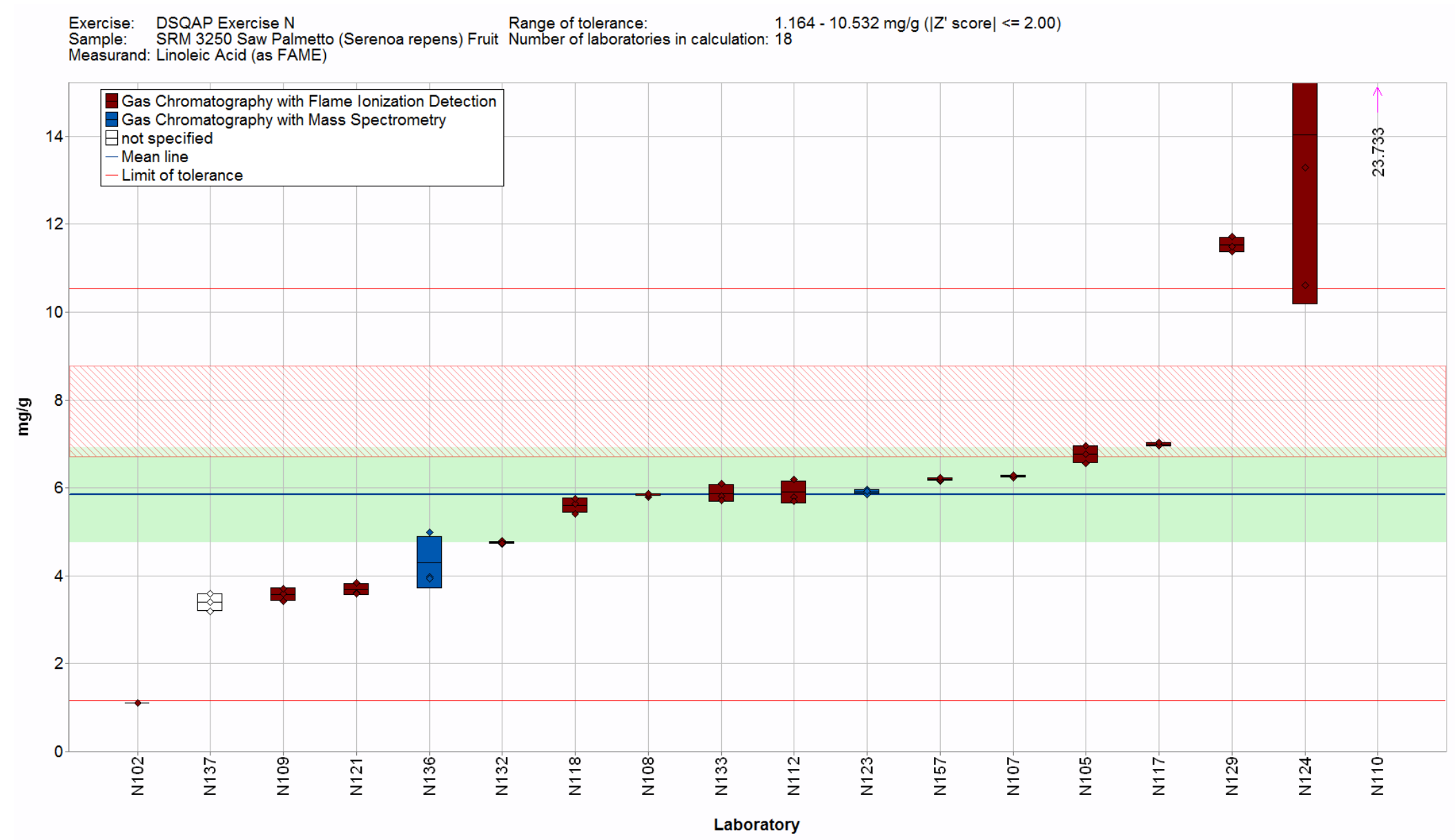

Figure 30. Linoleic acid (as FAME) in SRM 3250 Saw Palmetto (Serenoa repens) Fruit (data summary view - analytical method). In this view, individual laboratory data are plotted (diamonds) with the individual laboratory standard deviation (rectangle). The color of the data point represents the analytical method employed. The solid blue line represents the consensus mean and the green shaded region represents the $95 \%$ confidence interval for the consensus mean. The solid red lines represent the consensus range of tolerance, calculated as the values above and below the consensus mean that result in an acceptable $Z_{\text {comm }}^{\prime}$ score, $\left|Z_{\text {comm }}^{\prime}\right| \leq 2$. The red shaded region represents the NIST range of tolerance, which encompasses the NIST certified value bounded by twice its uncertainty ( $\left.U_{95}\right)$ and represents the range that results in an acceptable $Z_{\text {NIST }}$ score, $\left|Z_{\text {NIST }}\right| \leq 2$. 


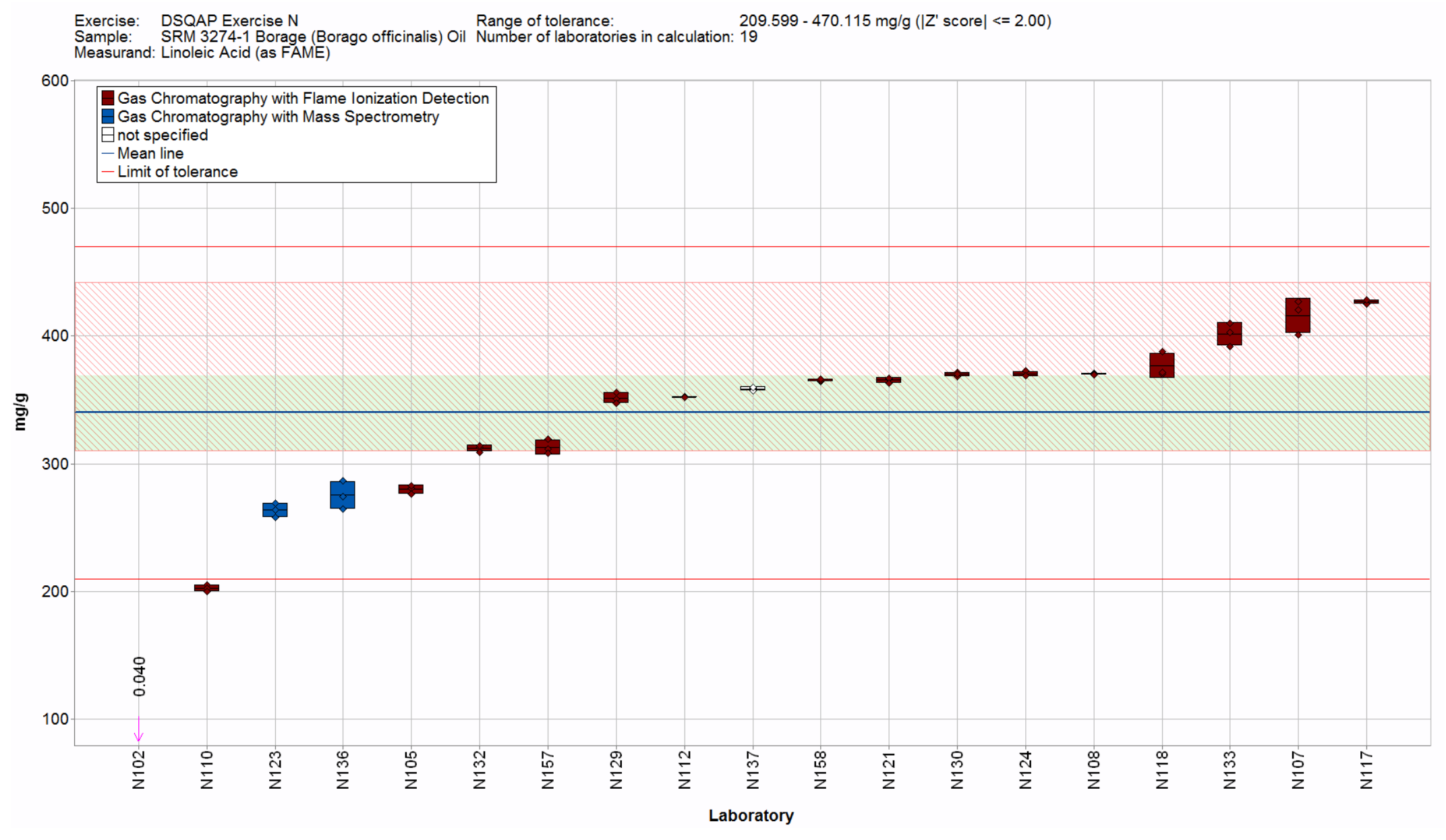

Figure 31. Linoleic acid (as FAME) in SRM 3274-1 Borage (Borago officinalis) Oil (data summary view - analytical method). In this view, individual laboratory data are plotted (diamonds) with the individual laboratory standard deviation (rectangle). The color of the data point represents the analytical method employed. The solid blue line represents the consensus mean and the green shaded region represents the $95 \%$ confidence interval for the consensus mean. The solid red lines represent the consensus range of tolerance, calculated as the values above and below the consensus mean that result in an acceptable $Z_{\text {comm }}^{\prime}$ score, $\left|Z_{\text {comm }}^{\prime}\right| \leq 2$. The red shaded region represents the NIST range of tolerance, which encompasses the NIST certified value bounded by twice its uncertainty (U95) and represents the range that results in an acceptable $Z_{\text {NIST }}$ score, $\left|Z_{\text {NIST }}\right| \leq 2$. 


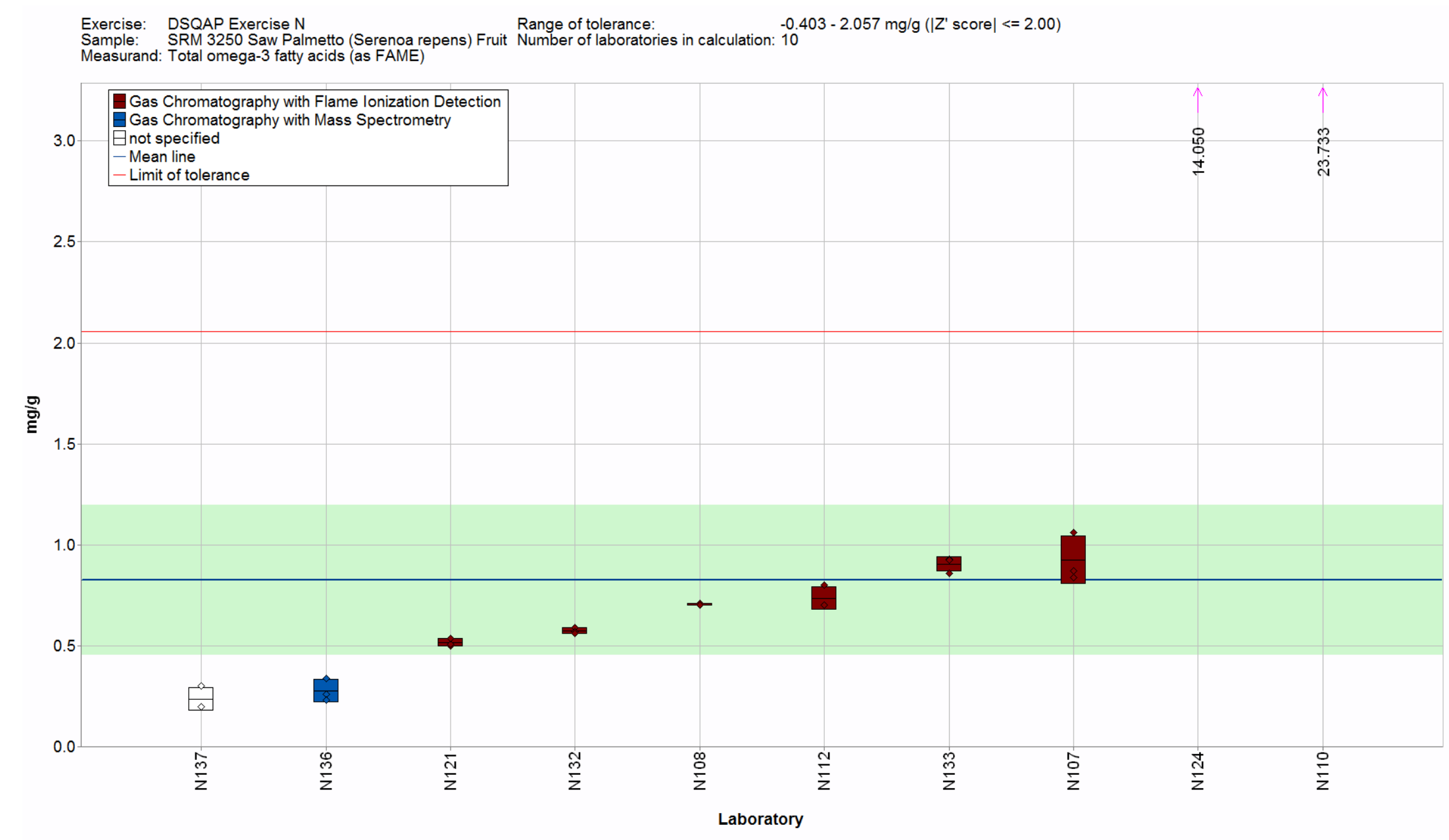

Figure 32. Total omega-3 fatty acids (as FAMEs) in SRM 3250 Saw Palmetto (Serenoa repens) Fruit (data summary view - analytical method). In this view, individual laboratory data are plotted (diamonds) with the individual laboratory standard deviation (rectangle). The color of the data point represents the analytical method employed. The solid blue line represents the consensus mean and the green shaded region represents the $95 \%$ confidence interval for the consensus mean. The solid red line represents the upper consensus range of tolerance, calculated as the values above and below the consensus mean that result in an acceptable $Z_{\text {comm }}^{\prime}$ score, $\left|Z_{\text {comm }}^{\prime}\right| \leq 2$, with the lower range set a zero. A NIST value has not been determined in this material. 


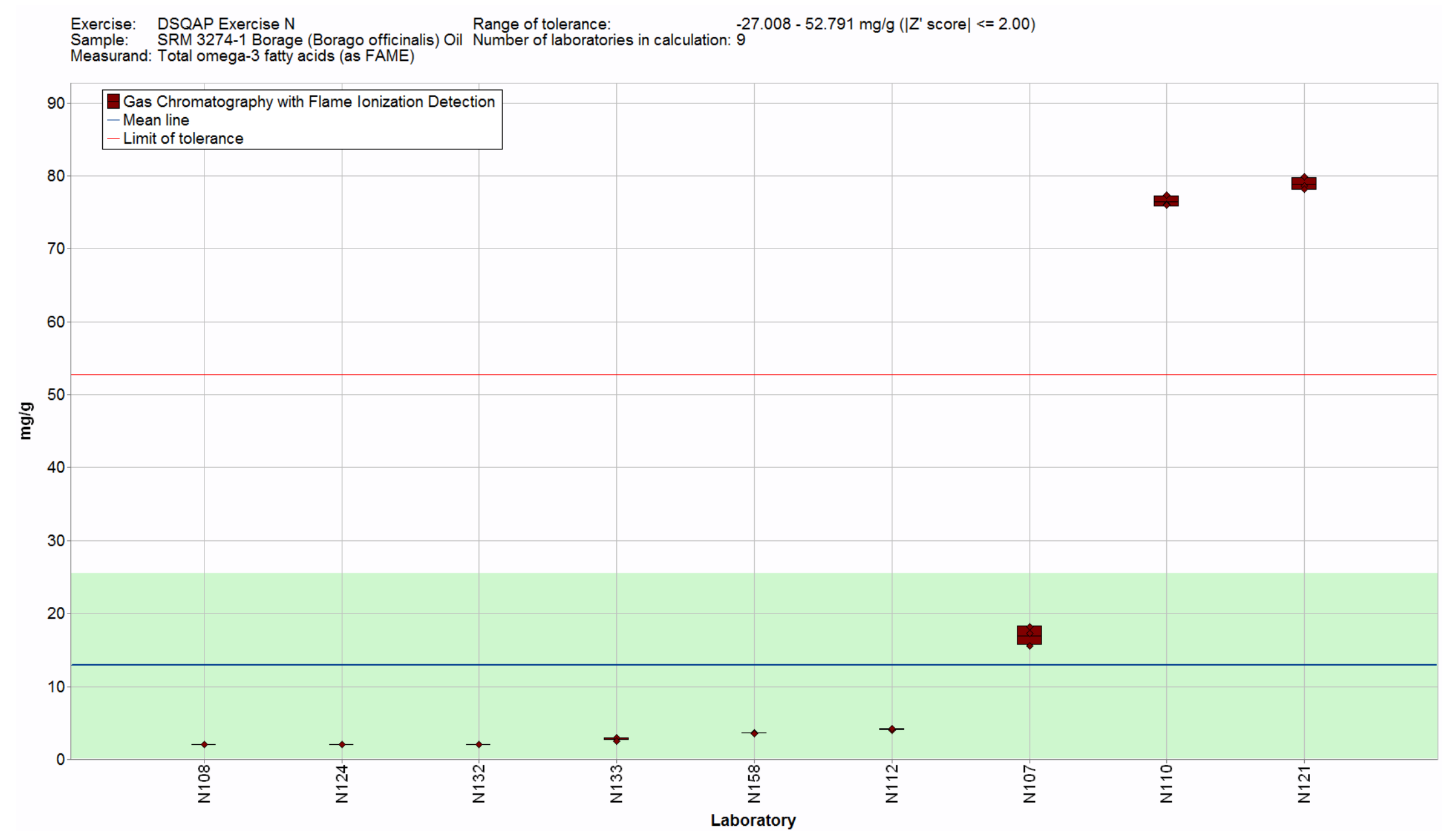

Figure 33. Total omega-3 fatty acids (as FAMEs) in SRM 3274-1 Borage (Borago officinalis) Oil (data summary view - analytical method). In this view, individual laboratory data are plotted (diamonds) with the individual laboratory standard deviation (rectangle). The color of the data point represents the analytical method employed. The solid blue line represents the consensus mean and the green shaded region represents the $95 \%$ confidence interval for the consensus mean. The solid red line represents the upper consensus range of tolerance, calculated as the values above and below the consensus mean that result in an acceptable $Z_{\text {comm }}^{\prime}$ score, $\left|Z_{\text {comm }}^{\prime}\right| \leq 2$, with the lower range set a zero. A NIST value has not been determined in this material. 

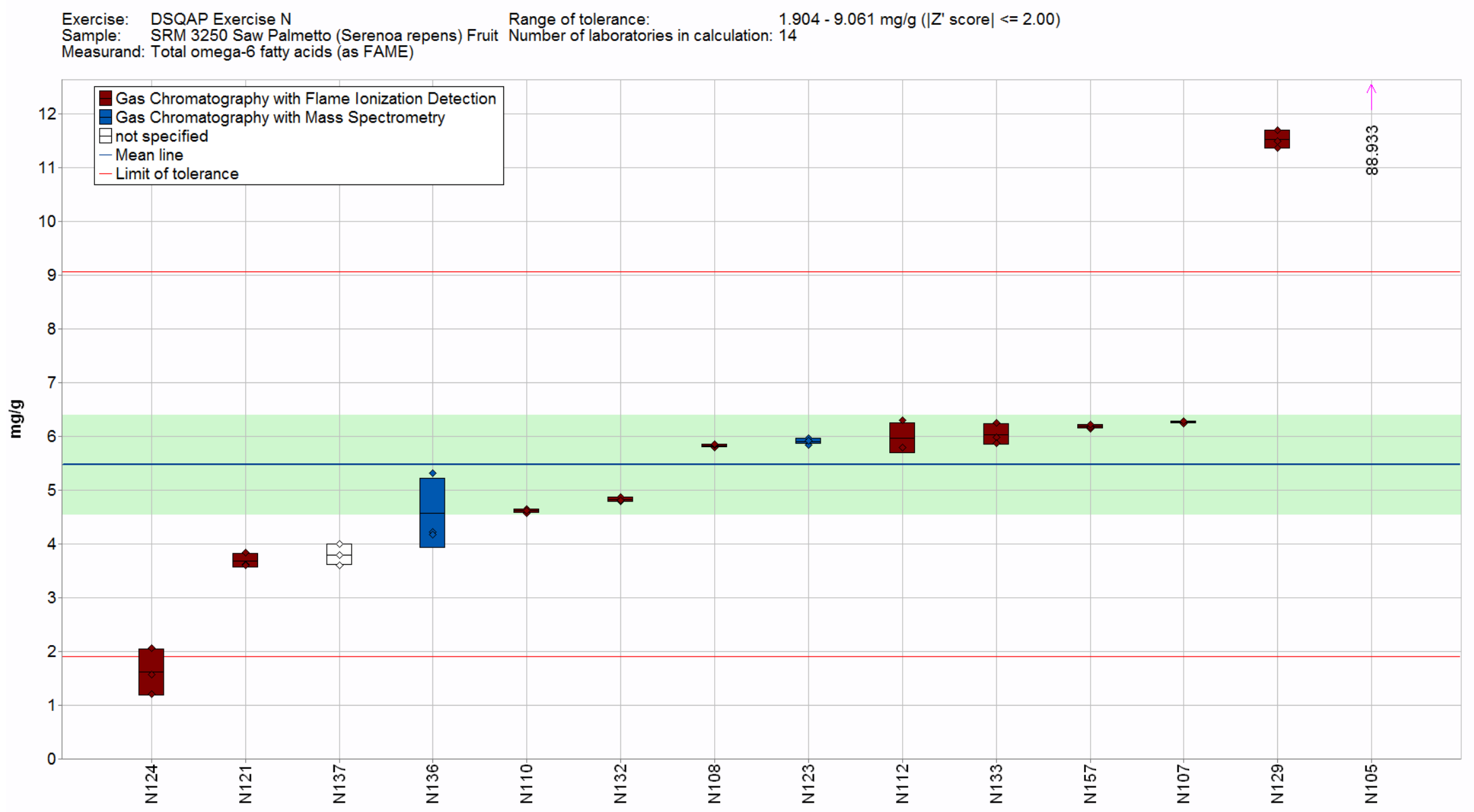

Figure 34. Total omega-6 fatty acids (as FAMEs) in SRM 3250 Saw Palmetto (Serenoa repens) Fruit (data summary view - analytical method). In this view, individual laboratory data are plotted (diamonds) with the individual laboratory standard deviation (rectangle). The color of the data point represents the analytical method employed. The solid blue line represents the consensus mean and the green shaded region represents the $95 \%$ confidence interval for the consensus mean. The solid red lines represent the consensus range of tolerance, calculated as the values above and below the consensus mean that result in an acceptable $Z_{\text {comm }}^{\prime}$ score, $\left|Z_{\text {comm }}^{\prime}\right| \leq 2$. A NIST value has not been determined in this material. A NIST value has not been determined in this material. 


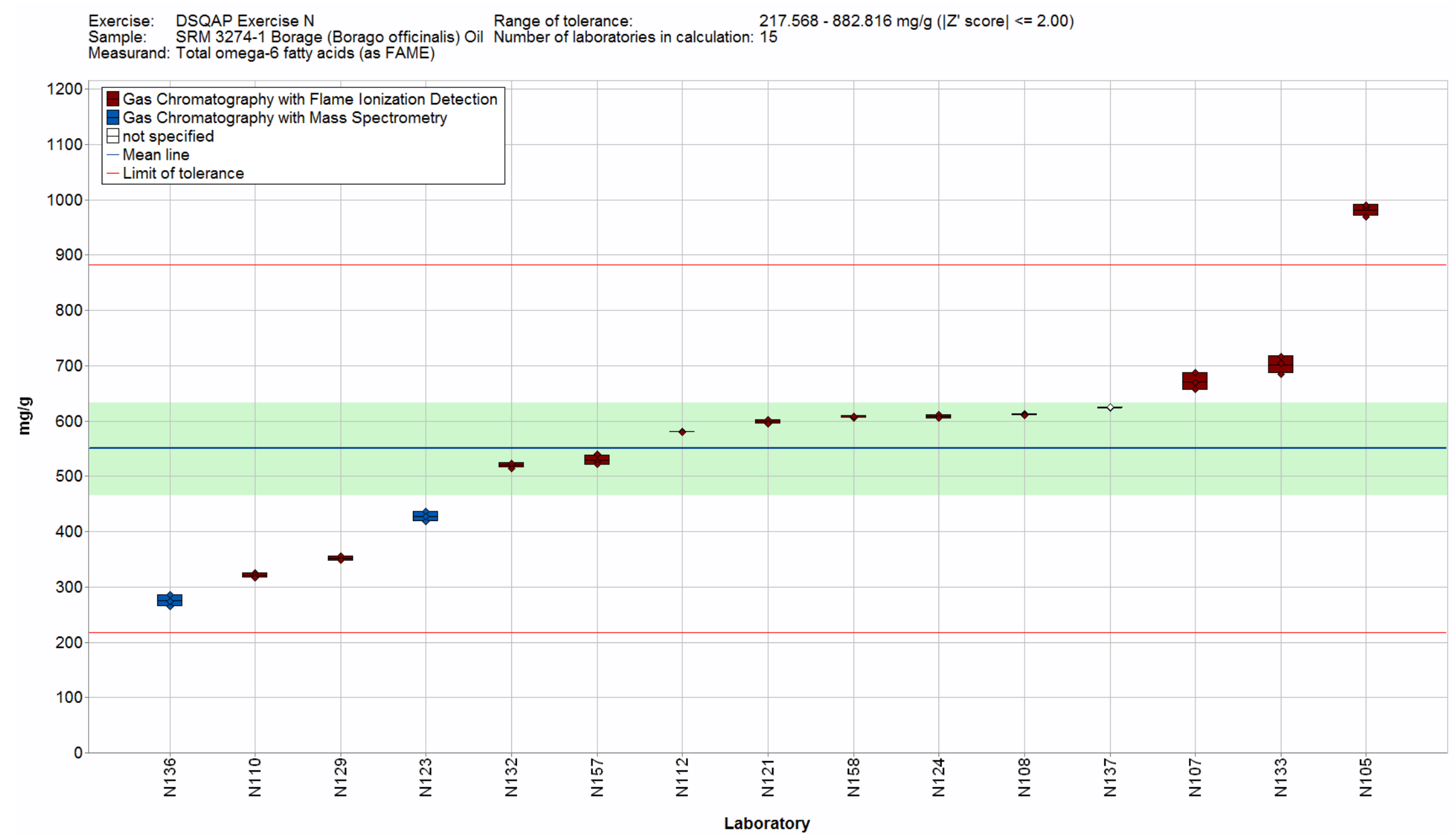

Figure 35. Total omega-6 fatty acid (as FAMEs) in SRM 3274-1 Borage (Borago officinalis) Oil (data summary view - analytical method). In this view, individual laboratory data are plotted (diamonds) with the individual laboratory standard deviation (rectangle). The color of the data point represents the analytical method employed. The solid blue line represents the consensus mean and the green shaded region represents the $95 \%$ confidence interval for the consensus mean. The solid red lines represent the consensus range of tolerance, calculated as the values above and below the consensus mean that result in an acceptable $Z_{\text {comm }}^{\prime}$ score, $\left|Z_{\text {comm }}^{\prime}\right| \leq 2$. A NIST value has not been determined in this material. 


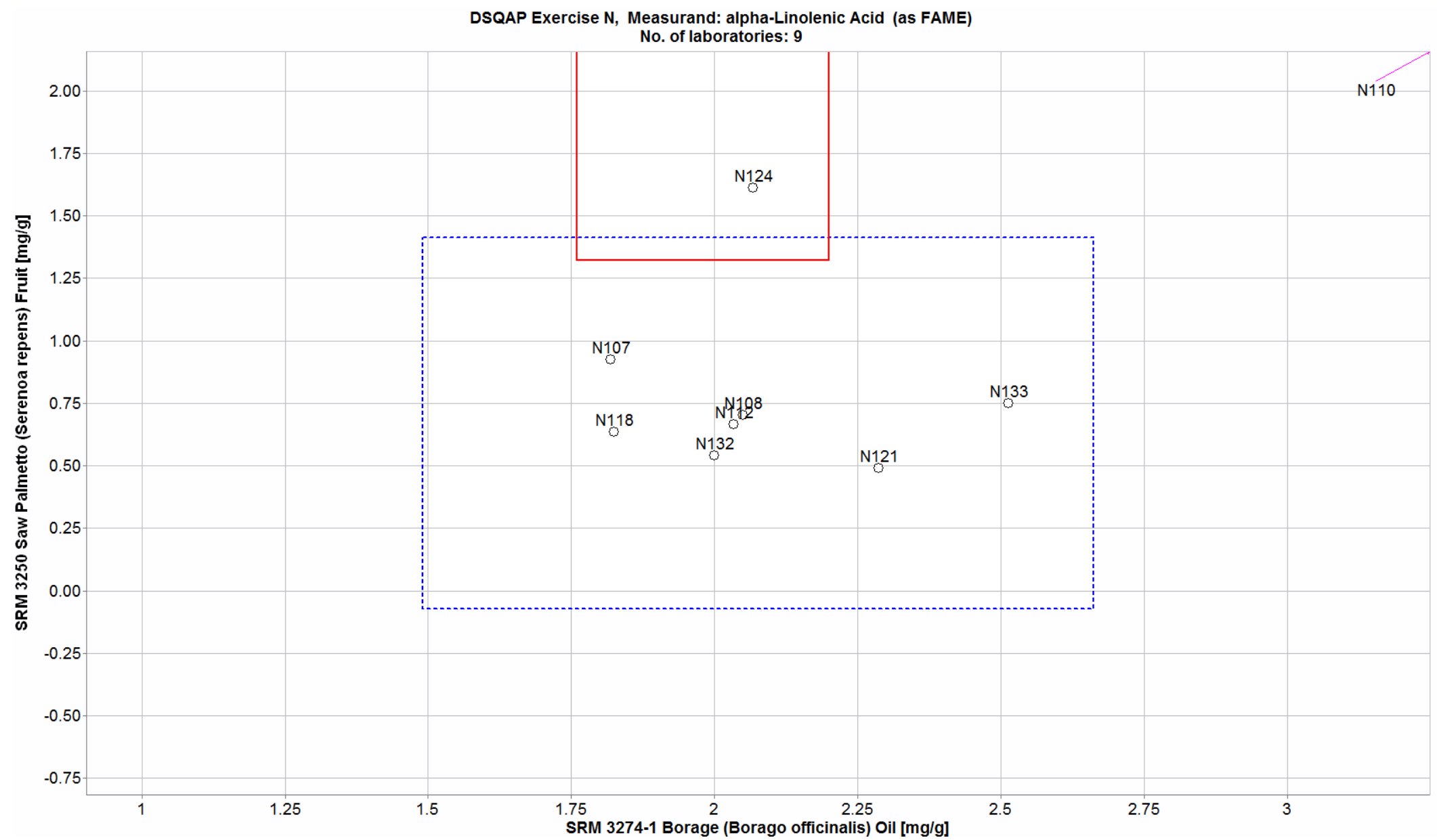

Figure 36. Laboratory means for $\alpha$-linolenic acid (as FAME) in SRM 3250 Saw Palmetto (Serenoa repens) Fruit, and SRM 3274-1 Borage (Borago officinalis) Oil (sample/sample comparison view). In this view, the individual laboratory mean for one sample (saw palmetto fruit) is compared to the mean for a second sample (borage oil). The solid red box represents the NIST range of tolerance for the two samples, borage oil (x-axis) and saw palmetto fruit (y-axis), which encompasses the NIST values bounded by their uncertainties $\left(U_{95}\right)$, and represents the range that results in an acceptable $Z_{N I S T}$ score, $\left|Z_{N I S T}\right| \leq 2$. The dotted blue box represents the consensus range of tolerance for borage oil (x-axis) and saw palmetto fruit (y-axis), calculated as the values above and below the consensus means that result in an acceptable $Z_{\text {comm }}^{\prime}$ score, $\left|Z_{\text {comm }}^{\prime}\right| \leq 2$. 
DSQAP Exercise N, Measurand: gamma-Linolenic Acid (as FAME)

No. of laboratories: 3

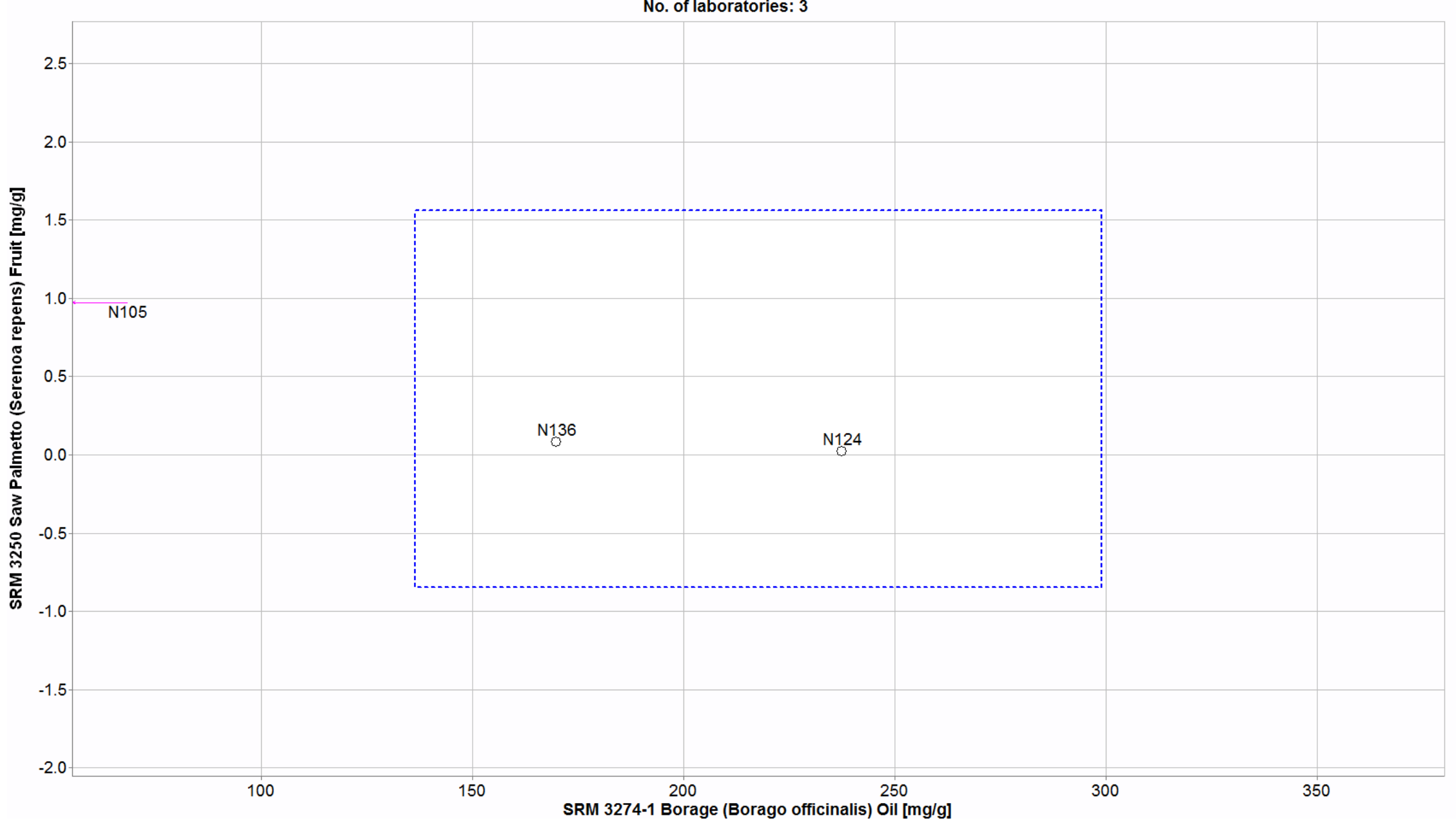

Figure 37. Laboratory means for $\gamma$-linolenic acid (as FAME) in SRM 3250 Saw Palmetto (Serenoa repens) Fruit, and SRM 3274-1 Borage (Borago officinalis) Oil (sample/sample comparison view). In this view, the individual laboratory mean for one sample (saw palmetto fruit) is compared to the mean for a second sample (borage oil). The dotted blue box represents the consensus range of tolerance for borage oil (x-axis) and saw palmetto fruit (y-axis), calculated as the values above and below the consensus means that result in an acceptable $Z_{\text {comm }}^{\prime}$ score, $\left|Z_{\text {comm }}^{\prime}\right| \leq 2$. 


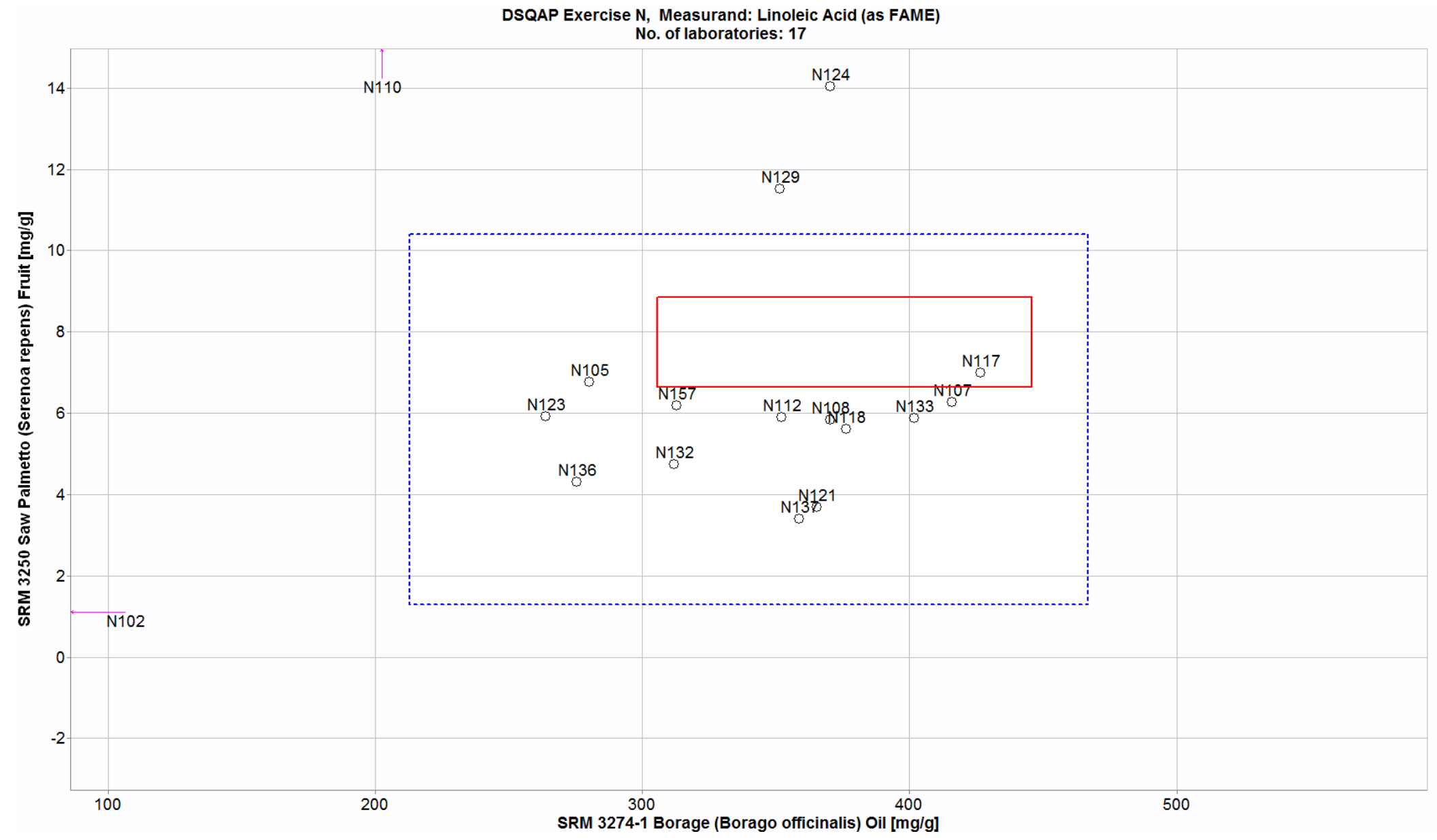

Figure 38. Laboratory means for linolenic acid (as FAME) in SRM 3250 Saw Palmetto (Serenoa repens) Fruit, and SRM 3274-1 Borage (Borago officinalis) Oil (sample/sample comparison view). In this view, the individual laboratory mean for one sample (saw palmetto fruit) is compared to the mean for a second sample (borage oil). The solid red box represents the NIST range of tolerance for the two samples, borage oil (x-axis) and saw palmetto fruit (y-axis), which encompasses the NIST values bounded by their uncertainties $\left(U_{95}\right)$, and represents the range that results in an acceptable $Z_{N I S T}$ score, $\left|Z_{N I S T}\right| \leq 2$. The dotted blue box represents the consensus range of tolerance for borage oil (x-axis) and saw palmetto fruit (y-axis), calculated as the values above and below the consensus means that result in an acceptable $Z_{\text {comm }}^{\prime}$ score, $\left|Z_{\text {comm }}^{\prime}\right| \leq 2$. 


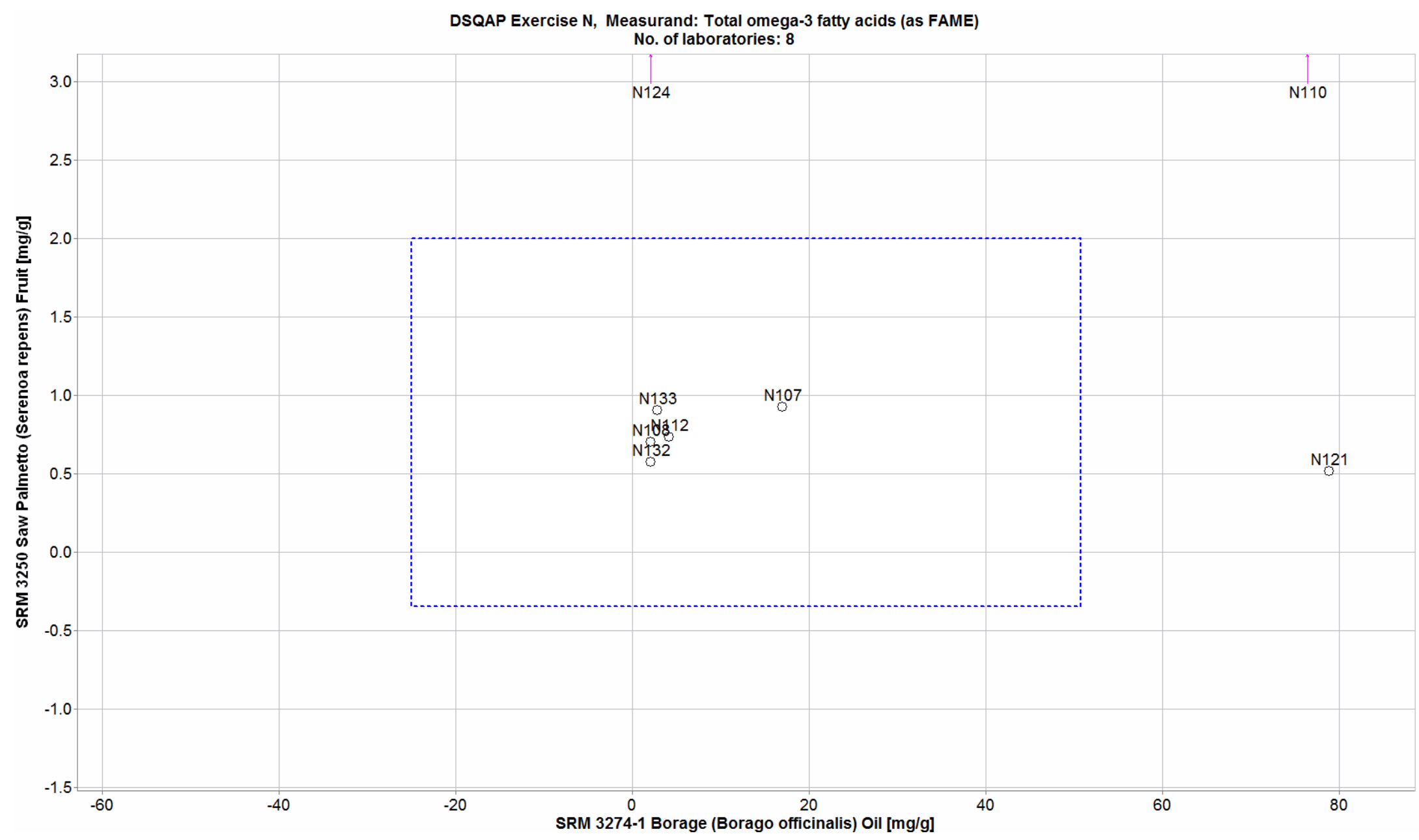

Figure 39. Laboratory means for total omega-3 fatty acids (as FAMEs) in SRM 3250 Saw Palmetto (Serenoa repens) Fruit, and SRM 3274-1 Borage (Borago officinalis) Oil (sample/sample comparison view). In this view, the individual laboratory mean for one sample (saw palmetto fruit) is compared to the mean for a second sample (borage oil). The dotted blue box represents the consensus range of tolerance for borage oil (x-axis) and saw palmetto fruit (y-axis), calculated as the values above and below the consensus means that result in an acceptable $Z_{\text {comm }}^{\prime}$ score, $\left|Z_{\text {comm }}^{\prime}\right| \leq 2$. 


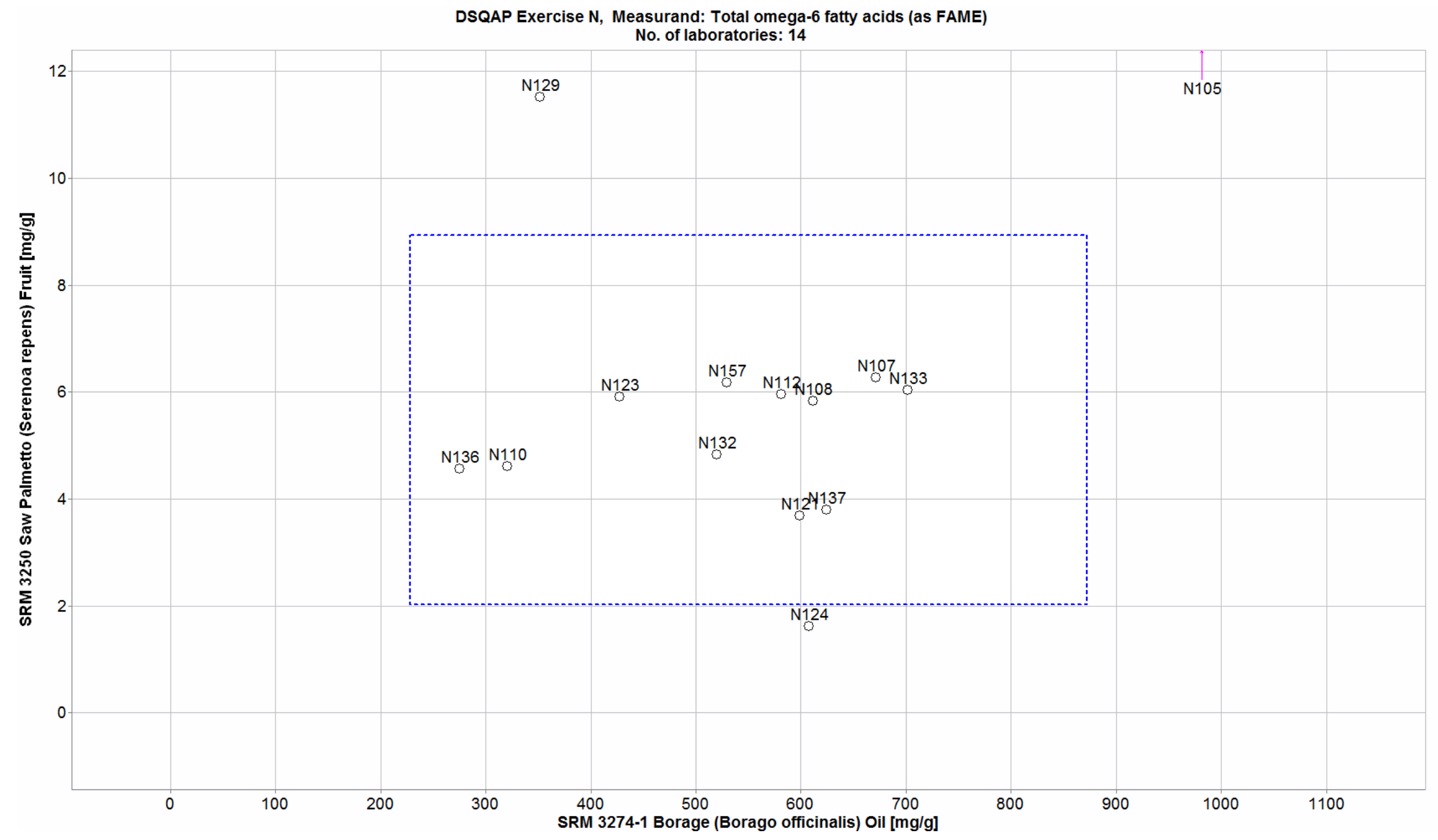

Figure 40. Laboratory means for total omega-6 fatty acids (as FAMEs) in SRM 3250 Saw Palmetto (Serenoa repens) Fruit, and SRM 3274-1 Borage (Borago officinalis) Oil (sample/sample comparison view). In this view, the individual laboratory mean for one sample (saw palmetto fruit) is compared to the mean for a second sample (borage oil). The dotted blue box represents the consensus range of tolerance for borage oil (x-axis) and saw palmetto fruit (y-axis), calculated as the values above and below the consensus means that result in an acceptable $Z_{\text {comm }}^{\prime}$ score, $\left|Z_{\text {comm }}^{\prime}\right| \leq 2$. 


\section{GINSENOSIDES IN GROUND ASIAN GINSENG EXTRACT AND RHIZOME BOTANICAL SUPPLEMENTS}

Study Overview

In this study, participants were provided with two candidate NIST SRMs, SRM 3384 Ground Asian Ginseng (Panax ginseng C.A. Meyer) Rhizome, and SRM 3385 Ground Asian Ginseng (Panax ginseng C.A. Meyer) Extract. Participants were asked to use in-house analytical methods to determine the mass fractions of ginsenosides Rb1, Rb2, Rc, Rd, Re, Rf, Rg1, and Rg2 in each of the matrices and report values in $\mathrm{mg} / \mathrm{g}$ on an as-received basis.

Sample Information

Ground Asian Ginseng Rhizome. Participants were provided with three packets, each containing approximately $3 \mathrm{~g}$ of ground ginseng rhizome. The rhizomes were ground, homogenized, and heat-sealed inside 4 mil polyethylene bags, which were then sealed inside nitrogen-flushed aluminized plastic bags along with two packets of silica gel. Before use, participants were instructed to thoroughly mix the contents of each packet and use a sample size of at least $1 \mathrm{~g}$. Participants were asked to store the material at controlled room temperature, $10{ }^{\circ} \mathrm{C}$ to $30{ }^{\circ} \mathrm{C}$, and to report a single value from each packet provided. Approximate analyte levels were not reported to participants prior to the study. NIST determined values for ginsenosides in candidate SRM 3384 using liquid chromatography with absorbance detection (LC-absorbance). The NIST-determined values and standard deviations are reported in the table below on an as-received basis.

\begin{tabular}{lr} 
Analyte & $\begin{array}{c}\text { NIST-Determined Mass Fraction in } \\
\text { SRM 3384 Ginseng Rhizome }(\mathrm{mg} / \mathrm{g})\end{array}$ \\
$\mathrm{Rb} 1$ & \multicolumn{2}{c}{ (as-received basis) } \\
$\mathrm{Rb} 2$ & $15.84 \pm 0.26$ \\
$\mathrm{Rc}$ & $9.30 \pm 0.10$ \\
$\mathrm{Rd}$ & $9.03 \pm 0.18$ \\
$\mathrm{Re}$ & $5.16 \pm 0.06$ \\
$\mathrm{Rf}$ & $6.61 \pm 0.14$ \\
$\mathrm{Rg} 1$ & $1.10 \pm 0.12$ \\
$\mathrm{Rg} 2$ & $3.94 \pm 0.08$
\end{tabular}

Ground Asian Ginseng Extract. Participants were provided with three packets, each containing approximately $1 \mathrm{~g}$ of ground ginseng extract. The extract was ground, homogenized, and heat-sealed inside 4 mil polyethylene bags, which were then sealed inside nitrogen-flushed aluminized plastic bags along with two packets of silica gel. Before use, participants were instructed to thoroughly mix the contents of each packet and use a sample size of at least $0.1 \mathrm{~g}$. Participants were asked to store the material at controlled room temperature, $10{ }^{\circ} \mathrm{C}$ to $30{ }^{\circ} \mathrm{C}$, and to report a single value from each packet provided. Approximate analyte levels were not reported to participants prior to the study, and target values for ginsenosides in candidate SRM 3385 have not been determined at NIST. 
Study Results

- Twenty-five laboratories enrolled in this exercise and received samples for all analytes. A summary of the participation rates is shown in the table below.

\begin{tabular}{ccc}
\cline { 2 - 3 } & \multicolumn{2}{c}{$\begin{array}{c}\text { Number of Laboratories Reporting } \\
\text { Results (Percent Participation) }\end{array}$} \\
\hline Analyte & $\begin{array}{c}\text { Asian ginseng } \\
\text { rhizome }\end{array}$ & $\begin{array}{c}\text { Asian ginseng } \\
\text { extract }\end{array}$ \\
\hline Rb1 & $16(64 \%)$ & $16(64 \%)$ \\
Rb2 & $15(60 \%)$ & $16(64 \%)$ \\
$\operatorname{Rc}$ & $15(60 \%)$ & $16(64 \%)$ \\
$\operatorname{Rd}$ & $15(60 \%)$ & $16(64 \%)$ \\
$\operatorname{Re}$ & $15(60 \%)$ & $16(64 \%)$ \\
$\operatorname{Rf}$ & $13(52 \%)$ & $13(52 \%)$ \\
$\operatorname{Rg} 1$ & $16(64 \%)$ & $16(64 \%)$ \\
$\operatorname{Rg} 2$ & $8(33 \%)$ & $8(33 \%)$ \\
\hline
\end{tabular}

- The consensus means for most ginsenosides in the Asian ginseng rhizome were below the target ranges and the consensus ranges did not overlap with the target ranges (see table below). The exceptions were ginsenosides $\mathrm{Rf}$ and $\mathrm{Rg} 2$. The between-laboratory variability is also summarized below, and ranged from good for $\mathrm{Rb} 1$ in the Asian ginseng extract $(11 \%)$ to very high for $\mathrm{Rg} 2$ in the Asian ginseng rhizome (90\%).

Between-Laboratory Variability

\begin{tabular}{cccc}
\hline Analyte & $\begin{array}{c}\text { Position of Consensus and Target in } \\
\text { Assian ginseng rhizome }\end{array}$ & $\begin{array}{c}\text { Asian ginseng } \\
\text { rhizome }\end{array}$ & $\begin{array}{c}\text { Asian ginseng } \\
\text { extract }\end{array}$ \\
\hline $\mathrm{Rb} 1$ & $\begin{array}{c}\text { Consensus mean below target range } \\
\text { Consensus range does not overlap target range } \\
\text { Consensus mean below target range }\end{array}$ & $16 \%$ & $11 \%$ \\
$\mathrm{Rb} 2$ & $\begin{array}{c}\text { Consensus range does not overlap target range } \\
\text { Consensus mean below target range }\end{array}$ & $20 \%$ & $19 \%$ \\
$\mathrm{Rc}$ & $\begin{array}{c}\text { Consensus range does not overlap target range } \\
\text { Consensus mean below target range }\end{array}$ & $21 \%$ & $21 \%$ \\
$\mathrm{Rd}$ & $\begin{array}{c}\text { Consensus range does not overlap target range } \\
\text { Consensus mean below target range }\end{array}$ & $11 \%$ & $13 \%$ \\
$\mathrm{Re}$ & $\begin{array}{c}\text { Consensus range does not overlap target range } \\
\text { Consensus mean within target range } \\
\text { Consensus range overlaps target range } \\
\text { Consensus mean below target range }\end{array}$ & $30 \%$ & $26 \%$ \\
$\mathrm{Rg} 1$ & $\begin{array}{c}\text { Consensus range does not overlap target range } \\
\text { Consensus mean above target range }\end{array}$ & $18 \%$ & $16 \%$ \\
$\mathrm{Rg} 2$ & Consensus range overlaps target range & $90 \%$ & $64 \%$ \\
\hline
\end{tabular}


- Most laboratories reported using LC with absorbance detection as their analytical method. One laboratory reported using an internal method (UPLC).

Technical Recommendations

The following recommendations are based on results obtained from the participants in this study.

- Most laboratories reported values below the NIST-determined value for most ginsenosides in the Asian ginseng rhizome samples. The low measured values may be a result of incomplete extraction of the ginsenosides from the sample matrix. To ensure maximum recovery, a subsequent re-extraction of the sample can indicate if measurable amounts of the analyte remain in the matrix following extraction.

- The sample/sample comparison graphs for nearly all of the ginsenosides demonstrate an upward trend, indicating a potential calibration issue. Whenever possible, a matched component should be used to quantify each of the ginsenosides. Calibrant purity should be carefully evaluated to identify and account for possible biases.

- When viewing Figures 57 through 64, an increasing trend in the sample/sample comparison data may be attributed to a potential calibration issue or greater difficulty with sample preparation of one sample over the second sample. This trend is very noticeable in ginsenosides $\mathrm{Rb} 1, \mathrm{Rb} 2, \mathrm{Rd}$, and $\mathrm{Re}$, and noticeable to a lesser degree in ginsenosides $\mathrm{Rc}, \mathrm{Rf}$, and $\operatorname{Rg} 2$. No noticeable trend in the data was observed for ginsenosides Rg1. 
Table 18. Individualized data summary table (NIST) for ginsenosides in botanical supplements.

\section{National Institute of Standards \& Technology}

\begin{tabular}{|c|c|c|c|c|c|c|}
\hline \multirow[b]{3}{*}{ Analyte } & \multirow[b]{2}{*}{ Lab Code: } & \multicolumn{5}{|c|}{ Exercise N - May 2017 - Ginsenosides } \\
\hline & & \multirow{2}{*}{$\begin{array}{l}\text { NIST } \\
\text { Units } \\
\end{array}$} & \multicolumn{4}{|c|}{ 1. Your Results } \\
\hline & Sample & & $\mathrm{x}_{\mathrm{i}}$ & $\mathrm{s}_{\mathrm{i}}$ & $Z_{\text {comm }}^{\prime}$ & $\mathrm{Z}_{\mathrm{NIST}}$ \\
\hline Ginsenoside $\mathrm{Rb} 1$ & Ginseng Rhizome & $\mathrm{mg} / \mathrm{g}$ & 15.84 & 0.26 & & 0.00 \\
\hline Ginsenoside $\mathrm{Rb} 1$ & Ginseng Extract & $\mathrm{mg} / \mathrm{g}$ & & & & \\
\hline Ginsenoside $\mathrm{Rb} 2$ & Ginseng Rhizome & $\mathrm{mg} / \mathrm{g}$ & 9.30 & 0.10 & & 0.00 \\
\hline Ginsenoside $\mathrm{Rb} 2$ & Ginseng Extract & $\mathrm{mg} / \mathrm{g}$ & & & & \\
\hline Ginsenoside Rc & Ginseng Rhizome & $\mathrm{mg} / \mathrm{g}$ & 9.03 & 0.18 & & 0.00 \\
\hline Ginsenoside Rc & Ginseng Extract & $\mathrm{mg} / \mathrm{g}$ & & & & \\
\hline Ginsenoside $\mathrm{Rd}$ & Ginseng Rhizome & $\mathrm{mg} / \mathrm{g}$ & 5.16 & 0.06 & & 0.00 \\
\hline Ginsenoside Rd & Ginseng Extract & $\mathrm{mg} / \mathrm{g}$ & & & & \\
\hline Ginsenoside $\operatorname{Re}$ & Ginseng Rhizome & $\mathrm{mg} / \mathrm{g}$ & 6.61 & 0.14 & & 0.00 \\
\hline Ginsenoside $\mathrm{Re}$ & Ginseng Extract & $\mathrm{mg} / \mathrm{g}$ & & & & \\
\hline Ginsenoside Rf & Ginseng Rhizome & $\mathrm{mg} / \mathrm{g}$ & 1.10 & 0.12 & & 0.00 \\
\hline Ginsenoside Rf & Ginseng Extract & $\mathrm{mg} / \mathrm{g}$ & & & & \\
\hline Ginsenoside Rg1 & Ginseng Rhizome & $\mathrm{mg} / \mathrm{g}$ & 3.94 & 0.08 & & 0.00 \\
\hline Ginsenoside Rg1 & Ginseng Extract & $\mathrm{mg} / \mathrm{g}$ & & & & \\
\hline Ginsenoside $\mathrm{Rg} 2$ & Ginseng Rhizome & $\mathrm{mg} / \mathrm{g}$ & 0.61 & 0.03 & & 0.00 \\
\hline Ginsenoside $\mathrm{Rg} 2$ & Ginseng Extract & $\mathrm{mg} / \mathrm{g}$ & & & & \\
\hline
\end{tabular}

$\mathrm{x}_{\mathrm{i}}$ Mean of reported values

$\mathrm{s}_{\mathrm{i}}$ Standard deviation of reported values

$Z_{\text {comm }}^{\prime} Z^{\prime}$-score with respect to community consensus

$\mathrm{Z}_{\text {NIST }} \mathrm{Z}$-score with respect to NIST value

\begin{tabular}{ccc}
\multicolumn{3}{c}{ 2. Community Results } \\
\hline $\mathrm{N}$ & $\mathrm{x}^{*}$ & $\mathrm{~s}^{*}$ \\
\hline 16 & 9.38 & 1.53 \\
16 & 34.2 & 3.9 \\
\hline 15 & 6.12 & 2.08 \\
16 & 20.1 & 4.3 \\
\hline 15 & 6.56 & 1.28 \\
16 & 26.8 & 5.0 \\
\hline 15 & 3.18 & 0.91 \\
16 & 14.9 & 3.2 \\
\hline 15 & 5.06 & 0.58 \\
16 & 17.0 & 2.2 \\
\hline 13 & 1.05 & 0.32 \\
13 & 2.92 & 0.77 \\
\hline 16 & 3.12 & 0.56 \\
16 & 6.27 & 1.03 \\
\hline 8 & 1.15 & 1.04 \\
8 & 4.43 & 2.85 \\
\hline
\end{tabular}

\begin{tabular}{cc}
\multicolumn{2}{c}{ 3. Target } \\
\hline $\mathrm{x}_{\mathrm{NIST}}$ & $U_{95}$ \\
\hline 15.84 & 0.26 \\
& \\
\hline 9.30 & 0.10 \\
\hline 9.03 & 0.18 \\
\hline 5.16 & 0.06 \\
\hline 6.61 & 0.14 \\
\hline 1.10 & 0.12 \\
\hline 3.94 & 0.08 \\
\hline 0.61 & 0.03 \\
\hline
\end{tabular}

$\mathrm{N}$ Number of quantitative

values reported

$x^{*}$ Robust mean of reported values

NIST NIST-assessed value

$U_{95} \pm 95 \%$ confidence interval about the assessed value or standard deviation $\left(\mathrm{s}_{\mathrm{NIST}}\right)$ 
Table 19. Data summary table for ginsenoside $\mathrm{Rb} 1$ in botanical supplements. Data highlighted in red have been flagged as potential outliers (e.g., difference from reference value, Grubb and/or Cochran) by the NIST software package.

\begin{tabular}{|c|c|c|c|c|c|c|c|c|c|c|c|}
\hline & \multirow[b]{3}{*}{ Lab } & \multicolumn{10}{|c|}{ Ginsenoside Rb1 } \\
\hline & & \multicolumn{5}{|c|}{ Ginseng Rhizome (mg/g) } & \multicolumn{5}{|c|}{ Ginseng Extract (mg/g) } \\
\hline & & $\mathbf{A}$ & $\mathbf{B}$ & $\mathbf{C}$ & Avg & SD & $\mathbf{A}$ & B & $\mathrm{C}$ & Avg & SD \\
\hline \multirow{26}{*}{ 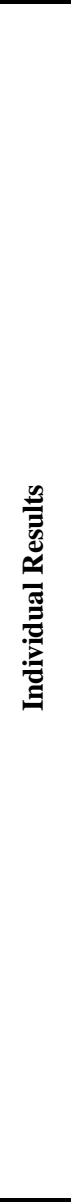 } & NIST & & & & 15.84 & 0.26 & & & & & \\
\hline & N105 & 10.50 & 10.90 & 10.50 & 10.63 & 0.23 & 37.3 & 37.8 & 37.7 & 37.6 & 0.3 \\
\hline & N107 & 10.34 & 10.68 & 10.52 & 10.51 & 0.17 & 37.6 & 37.8 & 38.2 & 37.9 & 0.3 \\
\hline & N108 & 6.36 & 6.34 & 6.09 & 6.26 & 0.15 & 27.5 & 26.7 & 26.8 & 27.0 & 0.5 \\
\hline & N109 & 8.78 & 8.66 & 8.94 & 8.79 & 0.14 & 35.0 & 34.7 & 35.0 & 34.9 & 0.2 \\
\hline & N110 & 7.73 & 7.46 & 7.60 & 7.60 & 0.14 & 32.0 & 31.8 & 31.3 & 31.7 & 0.4 \\
\hline & N111 & & & & & & & & & & \\
\hline & N115 & & & & & & & & & & \\
\hline & N117 & 7.85 & 7.84 & 7.26 & 7.65 & 0.34 & 30.4 & 30.1 & 28.4 & 29.6 & 1.1 \\
\hline & N118 & 9.44 & 9.46 & 9.33 & 9.41 & 0.07 & 32.0 & 34.5 & 31.9 & 32.8 & 1.5 \\
\hline & N120 & & & & & & & & & & \\
\hline & N121 & 9.61 & 9.52 & 9.50 & 9.54 & 0.06 & 33.9 & 34.2 & 33.9 & 34.0 & 0.2 \\
\hline & N122 & & & & & & & & & & \\
\hline & N124 & 13.48 & 13.61 & 13.49 & 13.53 & 0.07 & 44.6 & 44.7 & 44.4 & 44.6 & 0.2 \\
\hline & N125 & & & & & & & & & & \\
\hline & N128 & 10.06 & 10.03 & 9.96 & 10.02 & 0.05 & 34.1 & 33.8 & 33.3 & 33.7 & 0.4 \\
\hline & N129 & & & & & & & & & & \\
\hline & N130 & 9.32 & 9.56 & 9.37 & 9.42 & 0.13 & 35.7 & 34.3 & 34.0 & 34.7 & 0.9 \\
\hline & N131 & 13.18 & 13.34 & 13.25 & 13.26 & 0.08 & 47.8 & 48.2 & 47.2 & 47.7 & 0.5 \\
\hline & N132 & 9.73 & 9.48 & 9.60 & 9.60 & 0.13 & 34.7 & 34.8 & 34.7 & 34.7 & 0.1 \\
\hline & N134 & 8.41 & 8.11 & 7.92 & 8.15 & 0.25 & 29.9 & 30.4 & 30.0 & 30.1 & 0.3 \\
\hline & N141 & & & & & & & & & & \\
\hline & N146 & & & & & & & & & & \\
\hline & N154 & 9.18 & 9.34 & 9.18 & 9.23 & 0.09 & 33.8 & 33.3 & 33.1 & 33.4 & 0.3 \\
\hline & N155 & 9.00 & 9.21 & 9.09 & 9.10 & 0.11 & 33.5 & 33.7 & 33.8 & 33.7 & 0.2 \\
\hline & N158 & & & & & & & & & & \\
\hline \multirow{5}{*}{ 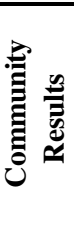 } & & \multicolumn{3}{|c|}{ Consensus Mean } & \multicolumn{2}{|l|}{9.38} & \multicolumn{2}{|c|}{ Consensus Mean } & & 34.2 & \\
\hline & & \multicolumn{3}{|c|}{ Consensus Standard Deviation } & \multicolumn{2}{|l|}{1.53} & \multicolumn{3}{|c|}{ Consensus Standard Deviation } & 3.9 & \\
\hline & & \multicolumn{3}{|c|}{ Maximum } & \multicolumn{2}{|l|}{13.53} & \multicolumn{3}{|c|}{ Maximum } & 47.7 & \\
\hline & & \multicolumn{3}{|c|}{ Minimum } & \multicolumn{2}{|l|}{6.26} & \multicolumn{3}{|c|}{ Minimum } & 27.0 & \\
\hline & & & \multicolumn{2}{|l|}{16} & \multicolumn{3}{|c|}{$\mathrm{N}$} & 16 & \\
\hline
\end{tabular}


Table 20. Data summary table for ginsenoside $\mathrm{Rb} 2$ in botanical supplements. Data highlighted in red have been flagged as potential outliers (e.g., difference from reference value, Grubb and/or Cochran) by the NIST software package.

\begin{tabular}{|c|c|c|c|c|c|c|c|c|c|c|c|}
\hline & \multirow[b]{3}{*}{ Lab } & \\
\hline & & \multicolumn{10}{|c|}{ Ginseng Rhizome (mg/g) } \\
\hline & & A & B & $\mathrm{C}$ & Avg & SD & $\mathbf{A}$ & B & $\mathrm{C}$ & Avg & SD \\
\hline \multirow{26}{*}{ 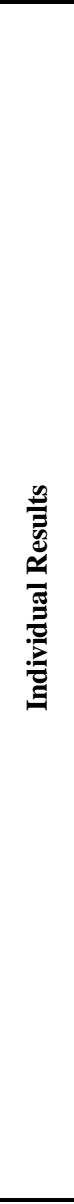 } & NIST & & & & 9.30 & 0.20 & & & & & \\
\hline & N105 & 5.36 & 5.87 & 5.63 & 5.62 & 0.26 & 19.8 & 20.9 & 18.6 & 19.8 & 1.2 \\
\hline & N107 & 8.64 & 9.04 & 8.80 & 8.83 & 0.20 & 28.5 & 27.6 & 28.5 & 28.2 & 0.5 \\
\hline & N108 & 1.17 & 1.11 & 1.07 & 1.12 & 0.05 & 5.8 & 5.5 & 5.0 & 5.4 & 0.4 \\
\hline & N109 & & & & & & 19.0 & 18.9 & 19.0 & 19.0 & 0.1 \\
\hline & N110 & 4.47 & 4.47 & 4.50 & 4.48 & 0.02 & 17.3 & 17.4 & 17.2 & 17.3 & 0.1 \\
\hline & N111 & & & & & & & & & & \\
\hline & N115 & & & & & & & & & & \\
\hline & N117 & 5.13 & 4.84 & 5.76 & 5.24 & 0.47 & 18.0 & 17.7 & 17.3 & 17.7 & 0.4 \\
\hline & N118 & 9.51 & 9.63 & 9.74 & 9.63 & 0.11 & 24.1 & 24.2 & 24.4 & 24.3 & 0.1 \\
\hline & N120 & & & & & & & & & & \\
\hline & N121 & 6.53 & 6.65 & 6.39 & 6.52 & 0.13 & 22.9 & 22.1 & 22.3 & 22.4 & 0.4 \\
\hline & N122 & & & & & & & & & & \\
\hline & N124 & 7.84 & 7.88 & 7.82 & 7.84 & 0.03 & 24.2 & 23.9 & 23.9 & 24.0 & 0.1 \\
\hline & N125 & & & & & & & & & & \\
\hline & N128 & 6.47 & 6.53 & 6.46 & 6.49 & 0.04 & 21.5 & 21.4 & 21.2 & 21.4 & 0.2 \\
\hline & N129 & & & & & & & & & & \\
\hline & N130 & 5.37 & 5.54 & 5.37 & 5.43 & 0.10 & 19.4 & 19.0 & 18.8 & 19.1 & 0.3 \\
\hline & N131 & 8.85 & 8.85 & 8.85 & 8.85 & 0.00 & 29.7 & 21.3 & 29.9 & 27.0 & 4.9 \\
\hline & N132 & 5.76 & 5.58 & 5.65 & 5.66 & 0.09 & 18.8 & 19.6 & 19.3 & 19.2 & 0.4 \\
\hline & N134 & 4.38 & 4.51 & 4.42 & 4.44 & 0.07 & 15.7 & 15.9 & 16.0 & 15.9 & 0.2 \\
\hline & N141 & & & & & & & & & & \\
\hline & N146 & & & & & & & & & & \\
\hline & N154 & 4.81 & 4.85 & 4.84 & 4.83 & 0.02 & 16.3 & 16.0 & 16.1 & 16.1 & 0.1 \\
\hline & N155 & 5.28 & 5.39 & 5.35 & 5.34 & 0.06 & 18.1 & 18.7 & 18.7 & 18.5 & 0.3 \\
\hline & N158 & & & & & & & & & & \\
\hline \multirow{5}{*}{ 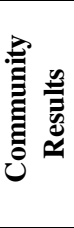 } & & \multicolumn{3}{|c|}{ Consensus Mean } & \multicolumn{2}{|l|}{6.12} & \multicolumn{2}{|c|}{ Consensus Mean } & \multicolumn{2}{|r|}{20.1} & \\
\hline & & \multicolumn{3}{|c|}{ Consensus Standard Deviation } & \multicolumn{2}{|l|}{2.08} & \multicolumn{3}{|c|}{ Consensus Standard Deviation } & 4.3 & \\
\hline & & \multicolumn{3}{|c|}{ Maximum } & \multicolumn{2}{|l|}{9.63} & \multicolumn{3}{|c|}{ Maximum } & 28.2 & \\
\hline & & \multicolumn{3}{|c|}{ Minimum } & \multicolumn{2}{|l|}{1.12} & \multicolumn{3}{|c|}{ Minimum } & 5.4 & \\
\hline & & & \multicolumn{2}{|l|}{15} & \multicolumn{3}{|c|}{$\mathrm{N}$} & 16 & \\
\hline
\end{tabular}


Table 21. Data summary table for ginsenoside Rc in botanical supplements. Data highlighted in red have been flagged as potential outliers (e.g., difference from reference value, Grubb and/or Cochran) by the NIST software package.

\begin{tabular}{|c|c|c|c|c|c|c|c|c|c|c|c|}
\hline & \multirow[b]{3}{*}{ Lab } & \multicolumn{10}{|c|}{ Ginsenoside Rc } \\
\hline & & \multicolumn{5}{|c|}{ Ginseng Rhizome (mg/g) } & \multicolumn{5}{|c|}{ Ginseng Extract $(\mathrm{mg} / \mathrm{g})$} \\
\hline & & $\mathbf{A}$ & B & $\mathbf{C}$ & Avg & SD & $\mathbf{A}$ & B & $\mathbf{C}$ & Avg & SD \\
\hline \multirow{26}{*}{ 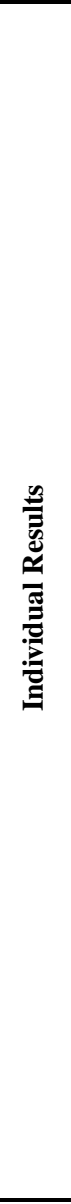 } & NIST & & & & 9.03 & 0.18 & & & & & \\
\hline & N105 & 6.20 & 6.40 & 6.08 & 6.23 & 0.16 & 27.5 & 28.0 & 27.5 & 27.7 & 0.3 \\
\hline & N107 & 7.44 & 7.58 & 7.44 & 7.49 & 0.08 & 27.8 & 28.0 & 28.3 & 28.1 & 0.3 \\
\hline & N108 & 5.57 & 5.37 & 5.59 & 5.51 & 0.12 & 27.7 & 27.2 & 29.0 & 28.0 & 0.9 \\
\hline & N109 & & & & & & 34.0 & 33.9 & 33.5 & 33.8 & 0.3 \\
\hline & N110 & 5.66 & 5.61 & 5.70 & 5.66 & 0.05 & 26.1 & 26.0 & 25.9 & 26.0 & 0.1 \\
\hline & N111 & & & & & & & & & & \\
\hline & N115 & & & & & & & & & & \\
\hline & N117 & 7.86 & 6.80 & 7.07 & 7.24 & 0.55 & 24.7 & 23.2 & 23.9 & 23.9 & 0.8 \\
\hline & N118 & 5.44 & 5.52 & 5.37 & 5.44 & 0.07 & 19.7 & 19.4 & 19.3 & 19.5 & 0.2 \\
\hline & N120 & & & & & & & & & & \\
\hline & N121 & 7.73 & 7.69 & 7.60 & 7.67 & 0.07 & 31.2 & 30.3 & 30.5 & 30.7 & 0.5 \\
\hline & N122 & & & & & & & & & & \\
\hline & N124 & 9.94 & 9.95 & 9.87 & 9.92 & 0.04 & 36.9 & 36.7 & 36.3 & 36.6 & 0.3 \\
\hline & N125 & & & & & & & & & & \\
\hline & N128 & 7.10 & 7.10 & 7.09 & 7.09 & 0.01 & 28.2 & 28.0 & 27.9 & 28.1 & 0.2 \\
\hline & N129 & & & & & & & & & & \\
\hline & N130 & 5.60 & 5.74 & 5.56 & 5.63 & 0.09 & 23.9 & 23.4 & 23.3 & 23.5 & 0.4 \\
\hline & N131 & 6.12 & 6.12 & 6.16 & 6.13 & 0.02 & 24.3 & 25.0 & 24.1 & 24.5 & 0.5 \\
\hline & N132 & 6.92 & 6.65 & 6.69 & 6.75 & 0.15 & 29.3 & 29.7 & 28.5 & 29.2 & 0.6 \\
\hline & N134 & 5.38 & 5.49 & 5.92 & 5.60 & 0.28 & 23.1 & 23.7 & 22.8 & 23.2 & 0.4 \\
\hline & N141 & & & & & & & & & & \\
\hline & N146 & & & & & & & & & & \\
\hline & N154 & 9.68 & 9.75 & 9.66 & 9.70 & 0.05 & 30.2 & 29.8 & 29.8 & 29.9 & 0.2 \\
\hline & N155 & 5.00 & 5.08 & 5.06 & 5.05 & 0.04 & 18.4 & 18.8 & 18.7 & 18.6 & 0.2 \\
\hline & N158 & & & & & & & & & & \\
\hline \multirow{5}{*}{ 象 } & & \multicolumn{3}{|c|}{ Consensus Mean } & \multicolumn{2}{|l|}{6.56} & \multicolumn{2}{|c|}{ Consensus Mean } & & 26.8 & \\
\hline & & \multicolumn{3}{|c|}{ Consensus Standard Deviation } & \multicolumn{2}{|l|}{1.28} & \multicolumn{3}{|c|}{ Consensus Standard Deviation } & 5.0 & \\
\hline & & \multicolumn{3}{|c|}{ Maximum } & \multicolumn{2}{|l|}{9.92} & \multicolumn{3}{|c|}{ Maximum } & 36.6 & \\
\hline & & \multicolumn{3}{|c|}{ Minimum } & \multicolumn{2}{|l|}{5.05} & \multicolumn{2}{|c|}{ Minimum } & & 18.6 & \\
\hline & & & \multicolumn{2}{|l|}{15} & \multicolumn{3}{|c|}{$\mathrm{N}$} & 16 & \\
\hline
\end{tabular}


Table 22. Data summary table for ginsenoside Rd in botanical supplements. Data highlighted in red have been flagged as potential outliers (e.g., difference from reference value, Grubb and/or Cochran) by the NIST software package.

\begin{tabular}{|c|c|c|c|c|c|c|c|c|c|c|c|}
\hline & \multirow[b]{3}{*}{ Lab } & \multicolumn{10}{|c|}{ Ginsenoside Rd } \\
\hline & & \multicolumn{5}{|c|}{ Ginseng Rhizome (mg/g) } & \multicolumn{5}{|c|}{ Ginseng Extract $(\mathrm{mg} / \mathrm{g})$} \\
\hline & & $\mathbf{A}$ & B & $\mathbf{C}$ & Avg & SD & $\mathbf{A}$ & B & $\mathbf{C}$ & Avg & SD \\
\hline \multirow{26}{*}{ 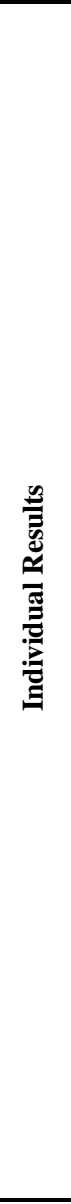 } & NIST & & & & 5.16 & 0.06 & & & & & \\
\hline & N105 & 2.58 & 2.64 & 2.91 & 2.71 & 0.18 & 13.9 & 14.2 & 13.7 & 13.9 & 0.3 \\
\hline & N107 & 3.75 & 3.75 & 3.65 & 3.72 & 0.06 & 16.8 & 16.7 & 16.7 & 16.7 & 0.0 \\
\hline & N108 & 5.86 & 5.60 & 5.74 & 5.74 & 0.13 & 27.7 & 27.6 & 25.5 & 26.9 & 1.2 \\
\hline & N109 & & & & & & 18.9 & 18.7 & 18.8 & 18.8 & 0.1 \\
\hline & N110 & 2.26 & 2.22 & 2.25 & 2.24 & 0.02 & 12.7 & 12.8 & 12.8 & 12.8 & 0.1 \\
\hline & N111 & & & & & & & & & & \\
\hline & N115 & & & & & & & & & & \\
\hline & N117 & 2.38 & 2.04 & 2.53 & 2.32 & 0.25 & 12.1 & 13.0 & 11.5 & 12.2 & 0.8 \\
\hline & N118 & 3.15 & 3.37 & 3.27 & 3.26 & 0.11 & 14.3 & 15.1 & 14.8 & 14.8 & 0.4 \\
\hline & N120 & & & & & & & & & & \\
\hline & N121 & 2.83 & 2.88 & 2.87 & 2.86 & 0.03 & 14.5 & 13.9 & 13.8 & 14.1 & 0.4 \\
\hline & N122 & & & & & & & & & & \\
\hline & N124 & 4.54 & 4.57 & 4.55 & 4.55 & 0.01 & 18.9 & 18.6 & 19.0 & 18.8 & 0.2 \\
\hline & N125 & & & & & & & & & & \\
\hline & N128 & 4.25 & 4.21 & 4.15 & 4.20 & 0.05 & 17.5 & 17.5 & 17.2 & 17.4 & 0.2 \\
\hline & N129 & & & & & & & & & & \\
\hline & N130 & 2.59 & 2.62 & 2.53 & 2.58 & 0.05 & 11.2 & 11.0 & 11.0 & 11.1 & 0.2 \\
\hline & N131 & 3.94 & 3.96 & 3.84 & 3.91 & 0.06 & 17.4 & 17.6 & 17.2 & 17.4 & 0.2 \\
\hline & N132 & 2.78 & 2.71 & 2.72 & 2.74 & 0.04 & 13.9 & 13.9 & 13.9 & 13.9 & 0.0 \\
\hline & N134 & 2.71 & 2.74 & 3.53 & 2.99 & 0.46 & 12.8 & 12.7 & 12.9 & 12.8 & 0.1 \\
\hline & N141 & & & & & & & & & & \\
\hline & N146 & & & & & & & & & & \\
\hline & N154 & 2.40 & 2.45 & 2.43 & 2.43 & 0.03 & 12.0 & 11.9 & 12.3 & 12.1 & 0.2 \\
\hline & N155 & 2.64 & 2.67 & 2.68 & 2.66 & 0.02 & 12.4 & 12.6 & 12.5 & 12.5 & 0.1 \\
\hline & N158 & & & & & & & & & & \\
\hline \multirow{5}{*}{ 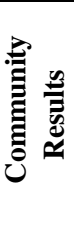 } & & \multicolumn{3}{|c|}{ Consensus Mean } & \multicolumn{2}{|l|}{3.18} & \multicolumn{2}{|c|}{ Consensus Mean } & & 14.9 & \\
\hline & & \multicolumn{3}{|c|}{ Consensus Standard Deviation } & \multicolumn{2}{|l|}{0.91} & \multicolumn{3}{|c|}{ Consensus Standard Deviation } & 3.2 & \\
\hline & & \multicolumn{3}{|c|}{ Maximum } & \multicolumn{2}{|l|}{5.74} & \multicolumn{3}{|c|}{ Maximum } & 26.9 & \\
\hline & & \multicolumn{3}{|c|}{ Minimum } & \multicolumn{2}{|l|}{2.24} & \multicolumn{2}{|c|}{ Minimum } & & 11.1 & \\
\hline & & & & \multicolumn{3}{|c|}{$\mathrm{N}$} & 16 & \\
\hline
\end{tabular}


Table 23. Data summary table for ginsenoside Re in botanical supplements. Data highlighted in red have been flagged as potential outliers (e.g., difference from reference value, Grubb and/or Cochran) by the NIST software package.

\begin{tabular}{|c|c|c|c|c|c|c|c|c|c|c|c|}
\hline & \multirow[b]{3}{*}{ Lab } & \multicolumn{10}{|c|}{ Ginsenoside $\mathrm{Re}$} \\
\hline & & \multicolumn{5}{|c|}{ Ginseng Rhizome (mg/g) } & \multicolumn{5}{|c|}{ Ginseng Extract $(\mathrm{mg} / \mathrm{g})$} \\
\hline & & $\mathbf{A}$ & B & $\mathbf{C}$ & Avg & SD & $\mathbf{A}$ & B & $\mathbf{C}$ & Avg & SD \\
\hline \multirow{26}{*}{ 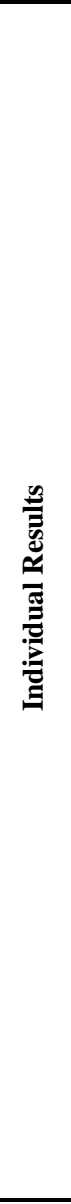 } & NIST & & & & 6.61 & 0.14 & & & & & \\
\hline & N105 & 5.35 & 5.56 & 5.34 & 5.42 & 0.12 & 17.3 & 17.6 & 17.6 & 17.5 & 0.2 \\
\hline & N107 & 5.05 & 5.03 & 5.00 & 5.03 & 0.02 & 17.1 & 16.8 & 17.1 & 17.0 & 0.2 \\
\hline & N108 & 1.38 & 1.20 & 0.97 & 1.19 & 0.20 & 2.9 & 2.5 & 2.3 & 2.6 & 0.3 \\
\hline & N109 & & & & & & 17.5 & 17.3 & 17.5 & 17.4 & 0.1 \\
\hline & N110 & 4.42 & 4.41 & 4.42 & 4.42 & 0.01 & 17.2 & 17.2 & 16.9 & 17.1 & 0.2 \\
\hline & N111 & & & & & & & & & & \\
\hline & N115 & & & & & & & & & & \\
\hline & N117 & 5.38 & 5.62 & 5.68 & 5.56 & 0.16 & 18.6 & 17.2 & 17.9 & 17.9 & 0.7 \\
\hline & N118 & 5.26 & 5.29 & 5.32 & 5.29 & 0.03 & 17.5 & 17.9 & 17.1 & 17.5 & 0.4 \\
\hline & N120 & & & & & & & & & & \\
\hline & N121 & 5.11 & 5.15 & 5.19 & 5.15 & 0.04 & 17.0 & 16.9 & 16.9 & 16.9 & 0.1 \\
\hline & N122 & & & & & & & & & & \\
\hline & N124 & 5.84 & 5.92 & 5.91 & 5.89 & 0.04 & 19.9 & 20.0 & 19.8 & 19.9 & 0.1 \\
\hline & N125 & & & & & & & & & & \\
\hline & N128 & 4.66 & 4.67 & 4.67 & 4.67 & 0.00 & 15.1 & 15.0 & 14.7 & 14.9 & 0.2 \\
\hline & N129 & & & & & & & & & & \\
\hline & N130 & 4.63 & 4.74 & 4.59 & 4.65 & 0.08 & 15.7 & 15.2 & 15.1 & 15.3 & 0.3 \\
\hline & N131 & 6.15 & 6.17 & 6.22 & 6.18 & 0.04 & 20.7 & 20.9 & 20.4 & 20.6 & 0.3 \\
\hline & N132 & 4.88 & 5.01 & 5.11 & 5.00 & 0.12 & 19.8 & 20.0 & 19.9 & 19.9 & 0.1 \\
\hline & N134 & 5.88 & 5.16 & 4.61 & 5.22 & 0.64 & 14.5 & 14.6 & 13.8 & 14.3 & 0.4 \\
\hline & N141 & & & & & & & & & & \\
\hline & N146 & & & & & & & & & & \\
\hline & N154 & 4.64 & 4.74 & 4.66 & 4.68 & 0.05 & 15.8 & 15.8 & 15.4 & 15.7 & 0.2 \\
\hline & N155 & 4.57 & 4.65 & 5.06 & 4.76 & 0.26 & 15.4 & 15.4 & 17.1 & 16.0 & 1.0 \\
\hline & N158 & & & & & & & & & & \\
\hline \multirow{5}{*}{ 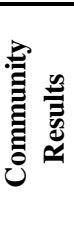 } & & \multicolumn{3}{|c|}{ Consensus Mean } & \multicolumn{2}{|l|}{5.06} & \multicolumn{2}{|c|}{ Consensus Mean } & & 17.0 & \\
\hline & & \multicolumn{3}{|c|}{ Consensus Standard Deviation } & \multicolumn{2}{|l|}{0.58} & \multicolumn{3}{|c|}{ Consensus Standard Deviation } & 2.2 & \\
\hline & & \multicolumn{3}{|c|}{ Maximum } & \multicolumn{2}{|l|}{6.18} & \multicolumn{3}{|c|}{ Maximum } & 20.6 & \\
\hline & & \multicolumn{3}{|c|}{ Minimum } & \multicolumn{2}{|l|}{1.19} & \multicolumn{3}{|c|}{ Minimum } & 2.6 & \\
\hline & & & \multicolumn{2}{|l|}{15} & & 16 & \\
\hline
\end{tabular}


Table 24. Data summary table for ginsenoside Rf in botanical supplements. Data highlighted in red have been flagged as potential outliers (e.g., difference from reference value, Grubb and/or Cochran) by the NIST software package.

\begin{tabular}{|c|c|c|c|c|c|c|c|c|c|c|c|}
\hline & \multirow[b]{3}{*}{ Lab } & \multicolumn{10}{|c|}{ Ginsenoside Rf } \\
\hline & & \multicolumn{5}{|c|}{ Ginseng Rhizome (mg/g) } & \multicolumn{5}{|c|}{ Ginseng Extract (mg/g) } \\
\hline & & $\mathbf{A}$ & B & $\mathbf{C}$ & Avg & SD & $\mathbf{A}$ & B & $\mathbf{C}$ & Avg & SD \\
\hline \multirow{26}{*}{ 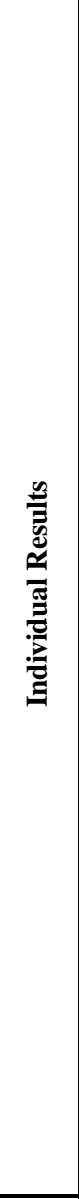 } & NIST & & & & 1.10 & 0.12 & & & & & \\
\hline & N105 & 0.89 & 0.93 & 0.93 & 0.92 & 0.03 & 2.62 & 2.59 & 2.65 & 2.62 & 0.03 \\
\hline & N107 & 0.80 & 0.85 & 0.80 & 0.82 & 0.03 & 2.62 & 2.62 & 2.58 & 2.61 & 0.02 \\
\hline & N108 & 1.06 & 1.07 & 0.83 & 0.99 & 0.14 & 3.43 & 3.45 & 3.52 & 3.47 & 0.04 \\
\hline & N109 & & & & & & & & & & \\
\hline & N110 & 1.42 & 1.35 & 1.25 & 1.34 & 0.09 & 4.39 & 4.80 & 4.68 & 4.62 & 0.21 \\
\hline & N111 & & & & & & & & & & \\
\hline & N115 & & & & & & & & & & \\
\hline & N117 & 1.64 & 1.41 & 1.42 & 1.49 & 0.13 & 3.43 & 3.60 & 2.83 & 3.29 & 0.40 \\
\hline & N118 & 1.75 & 1.61 & 1.58 & 1.64 & 0.09 & 3.72 & 3.14 & 4.15 & 3.67 & 0.51 \\
\hline & N120 & & & & & & & & & & \\
\hline & N121 & & & & & & & & & & \\
\hline & N122 & & & & & & & & & & \\
\hline & N124 & 1.28 & 1.25 & 1.24 & 1.26 & 0.02 & 3.63 & 3.41 & 3.35 & 3.46 & 0.15 \\
\hline & N125 & & & & & & & & & & \\
\hline & N128 & 1.19 & 1.22 & 1.21 & 1.21 & 0.02 & 3.50 & 3.51 & 3.43 & 3.48 & 0.04 \\
\hline & N129 & & & & & & & & & & \\
\hline & N130 & 0.95 & 0.98 & 0.76 & 0.90 & 0.12 & 1.99 & 1.92 & 2.06 & 1.99 & 0.07 \\
\hline & N131 & & & & & & & & & & \\
\hline & N132 & 0.78 & 0.75 & 0.74 & 0.76 & 0.02 & 2.16 & 2.51 & 2.57 & 2.41 & 0.22 \\
\hline & N134 & 0.80 & 0.77 & 0.72 & 0.77 & 0.04 & 2.16 & 2.03 & 2.05 & 2.08 & 0.07 \\
\hline & N141 & & & & & & & & & & \\
\hline & N146 & & & & & & & & & & \\
\hline & N154 & 0.82 & 0.84 & 0.82 & 0.83 & 0.01 & 2.33 & 2.38 & 2.31 & 2.34 & 0.04 \\
\hline & N155 & 0.86 & 0.87 & 0.87 & 0.87 & 0.01 & 2.49 & 2.41 & 2.45 & 2.45 & 0.04 \\
\hline & N158 & & & & & & & & & & \\
\hline \multirow{5}{*}{ 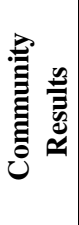 } & & \multicolumn{3}{|c|}{ Consensus Mean } & \multicolumn{2}{|l|}{1.05} & \multicolumn{2}{|c|}{ Consensus Mean } & & 2.92 & \\
\hline & & \multicolumn{3}{|c|}{ Consensus Standard Deviation } & \multicolumn{2}{|l|}{0.32} & \multicolumn{3}{|c|}{ Consensus Standard Deviation } & 0.77 & \\
\hline & & \multicolumn{3}{|c|}{ Maximum } & \multicolumn{2}{|l|}{1.64} & \multicolumn{3}{|c|}{ Maximum } & 4.62 & \\
\hline & & \multicolumn{3}{|c|}{ Minimum } & \multicolumn{2}{|l|}{0.76} & \multicolumn{3}{|c|}{ Minimum } & 1.99 & \\
\hline & & \multicolumn{3}{|l|}{$\mathrm{N}$} & \multicolumn{2}{|l|}{13} & \multicolumn{3}{|l|}{$\mathrm{N}$} & 13 & \\
\hline
\end{tabular}


Table 25. Data summary table for ginsenoside Rg1 in botanical supplements. Data highlighted in red have been flagged as potential outliers (e.g., difference from reference value, Grubb and/or Cochran) by the NIST software package.

\begin{tabular}{|c|c|c|c|c|c|c|c|c|c|c|c|}
\hline & \multirow[b]{3}{*}{ Lab } & \multicolumn{10}{|c|}{ Ginsenoside Rg1 } \\
\hline & & \multicolumn{5}{|c|}{ Ginseng Rhizome (mg/g) } & \multicolumn{5}{|c|}{ Ginseng Extract (mg/g) } \\
\hline & & $\mathbf{A}$ & $\mathbf{B}$ & $\mathbf{C}$ & Avg & SD & $\mathbf{A}$ & B & $\mathbf{C}$ & Avg & SD \\
\hline \multirow{26}{*}{ 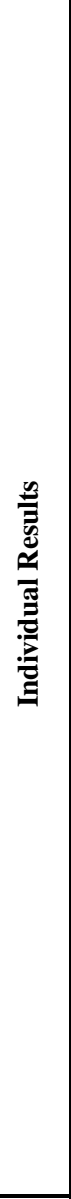 } & NIST & & & & 3.94 & 0.08 & & & & & \\
\hline & N105 & 3.47 & 3.60 & 3.50 & 3.52 & 0.07 & 6.88 & 7.22 & 1.31 & 5.14 & 3.32 \\
\hline & N107 & 2.92 & 2.90 & 2.86 & 2.89 & 0.03 & 6.30 & 6.23 & 6.23 & 6.25 & 0.04 \\
\hline & N108 & 3.74 & 4.18 & 4.33 & 4.08 & 0.31 & 13.84 & 14.04 & 15.99 & 14.62 & 1.19 \\
\hline & N109 & 2.73 & 2.68 & 2.79 & 2.73 & 0.06 & 6.27 & 6.22 & 6.25 & 6.25 & 0.03 \\
\hline & N110 & 2.51 & 2.51 & 2.55 & 2.52 & 0.02 & 6.46 & 6.45 & 6.70 & 6.54 & 0.14 \\
\hline & N111 & & & & & & & & & & \\
\hline & N115 & & & & & & & & & & \\
\hline & N117 & 2.74 & 2.52 & 3.29 & 2.85 & 0.40 & 5.61 & 5.98 & 6.36 & 5.98 & 0.38 \\
\hline & N118 & 3.01 & 3.34 & 3.19 & 3.18 & 0.16 & 7.13 & 7.00 & 7.25 & 7.13 & 0.13 \\
\hline & N120 & & & & & & & & & & \\
\hline & N121 & 2.68 & 2.71 & 2.63 & 2.67 & 0.04 & 5.62 & 5.46 & 5.64 & 5.57 & 0.10 \\
\hline & N122 & & & & & & & & & & \\
\hline & N124 & 3.66 & 3.72 & 3.71 & 3.69 & 0.03 & 7.85 & 7.85 & 7.85 & 7.85 & 0.00 \\
\hline & N125 & & & & & & & & & & \\
\hline & N128 & 3.07 & 3.07 & 3.09 & 3.08 & 0.01 & 6.41 & 6.37 & 6.27 & 6.35 & 0.07 \\
\hline & N129 & & & & & & & & & & \\
\hline & N130 & 2.66 & 2.77 & 2.63 & 2.69 & 0.07 & 5.33 & 5.33 & 5.20 & 5.29 & 0.08 \\
\hline & N131 & 3.70 & 3.70 & 3.74 & 3.71 & 0.02 & 6.63 & 6.68 & 6.54 & 6.62 & 0.07 \\
\hline & N132 & 2.77 & 2.76 & 2.81 & 2.78 & 0.03 & 7.14 & 7.14 & 7.13 & 7.14 & 0.01 \\
\hline & N134 & 4.25 & 4.26 & 4.05 & 4.19 & 0.12 & 4.62 & 4.57 & 5.15 & 4.78 & 0.32 \\
\hline & N141 & & & & & & & & & & \\
\hline & N146 & & & & & & & & & & \\
\hline & N154 & 2.57 & 2.59 & 2.57 & 2.58 & 0.01 & 5.44 & 5.38 & 5.20 & 5.34 & 0.12 \\
\hline & N155 & 3.01 & 3.02 & 3.01 & 3.01 & 0.01 & 6.30 & 6.27 & 6.26 & 6.28 & 0.02 \\
\hline & N158 & & & & & & & & & & \\
\hline \multirow{5}{*}{ 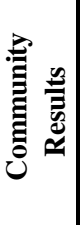 } & & \multicolumn{3}{|c|}{ Consensus Mean } & \multicolumn{2}{|l|}{3.12} & \multicolumn{2}{|c|}{ Consensus Mean } & & 6.27 & \\
\hline & & \multicolumn{3}{|c|}{ Consensus Standard Deviation } & \multicolumn{2}{|l|}{0.56} & \multicolumn{3}{|c|}{ Consensus Standard Deviation } & 1.03 & \\
\hline & & \multicolumn{3}{|c|}{ Maximum } & \multicolumn{2}{|l|}{4.19} & \multicolumn{3}{|c|}{ Maximum } & 14.62 & \\
\hline & & \multicolumn{3}{|c|}{ Minimum } & \multicolumn{2}{|l|}{2.52} & \multicolumn{3}{|c|}{ Minimum } & 4.78 & \\
\hline & & \multicolumn{3}{|l|}{$\mathrm{N}$} & \multicolumn{2}{|l|}{16} & \multicolumn{3}{|l|}{$\mathrm{N}$} & 16 & \\
\hline
\end{tabular}


Table 26. Data summary table for ginsenoside $\operatorname{Rg} 2$ in botanical supplements. Data highlighted in red have been flagged as potential outliers (e.g., difference from reference value, Grubb and/or Cochran) by the NIST software package.

\begin{tabular}{|c|c|c|c|c|c|c|c|c|c|c|c|}
\hline & \multirow[b]{3}{*}{ Lab } & \multicolumn{10}{|c|}{ Ginsenoside Rg2 } \\
\hline & & \multicolumn{5}{|c|}{ Ginseng Rhizome (mg/g) } & \multicolumn{5}{|c|}{ Ginseng Extract (mg/g) } \\
\hline & & $\mathbf{A}$ & B & $\mathbf{C}$ & Avg & SD & $\mathbf{A}$ & B & $\mathbf{C}$ & Avg & SD \\
\hline \multirow{25}{*}{ 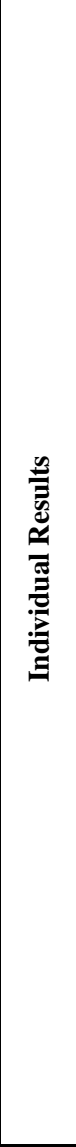 } & NIST & & & & 0.61 & 0.03 & & & & & \\
\hline & N105 & 3.47 & 3.60 & 3.50 & 3.52 & 0.07 & 6.88 & 7.22 & 7.31 & 7.14 & 0.23 \\
\hline & N107 & 0.68 & 0.63 & 0.60 & 0.63 & 0.04 & 2.54 & 2.55 & 2.53 & 2.54 & 0.01 \\
\hline & N108 & 2.54 & 2.39 & 2.44 & 2.46 & 0.08 & 16.30 & 15.88 & 14.97 & 15.72 & 0.68 \\
\hline & N109 & & & & & & & & & & \\
\hline & N110 & 0.82 & 0.74 & 0.74 & 0.77 & 0.05 & 3.91 & 3.90 & 3.87 & 3.89 & 0.02 \\
\hline & N111 & & & & & & & & & & \\
\hline & N115 & & & & & & & & & & \\
\hline & N118 & 1.14 & 1.23 & 1.14 & 1.17 & 0.05 & 5.83 & 6.00 & 6.05 & 5.96 & 0.11 \\
\hline & N120 & & & & & & & & & & \\
\hline & N121 & & & & & & & & & & \\
\hline & N122 & & & & & & & & & & \\
\hline & N124 & & & & & & & & & & \\
\hline & N125 & & & & & & & & & & \\
\hline & N128 & $<1.000$ & $<1.000$ & $<1.000$ & $<1.000$ & & $<1.000$ & $<1.000$ & $<1.000$ & $<1.000$ & \\
\hline & N129 & & & & & & & & & & \\
\hline & N130 & & & & & & & & & & \\
\hline & N131 & & & & & & & & & & \\
\hline & N132 & & & & & & & & & & \\
\hline & N134 & 0.43 & 0.35 & 0.35 & 0.38 & 0.04 & 2.40 & 2.54 & 2.59 & 2.51 & 0.10 \\
\hline & N141 & & & & & & & & & & \\
\hline & N146 & & & & & & & & & & \\
\hline & N154 & 0.54 & 0.54 & 0.54 & 0.54 & 0.00 & 2.50 & 2.47 & 2.37 & 2.45 & 0.07 \\
\hline & N155 & 0.61 & 0.48 & 0.49 & 0.53 & 0.07 & 2.23 & 2.28 & 2.29 & 2.27 & 0.03 \\
\hline & N158 & & & & & & & & & & \\
\hline \multirow{5}{*}{ 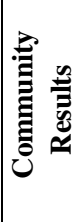 } & & \multicolumn{3}{|c|}{ Consensus Mean } & \multicolumn{2}{|l|}{1.15} & \multicolumn{2}{|c|}{ Consensus Mean } & & 4.43 & \\
\hline & & \multicolumn{3}{|c|}{ Consensus Standard Deviation } & \multicolumn{2}{|l|}{1.04} & \multicolumn{3}{|c|}{ Consensus Standard Deviation } & 2.85 & \\
\hline & & \multicolumn{3}{|l|}{ Maximum } & \multicolumn{2}{|l|}{3.52} & \multicolumn{3}{|l|}{ Maximum } & 15.72 & \\
\hline & & \multicolumn{3}{|l|}{ Minimum } & \multicolumn{2}{|l|}{0.38} & \multicolumn{3}{|l|}{ Minimum } & 2.27 & \\
\hline & & \multicolumn{3}{|l|}{$\mathrm{N}$} & \multicolumn{2}{|l|}{8} & \multicolumn{3}{|l|}{$\mathrm{N}$} & 8 & \\
\hline
\end{tabular}




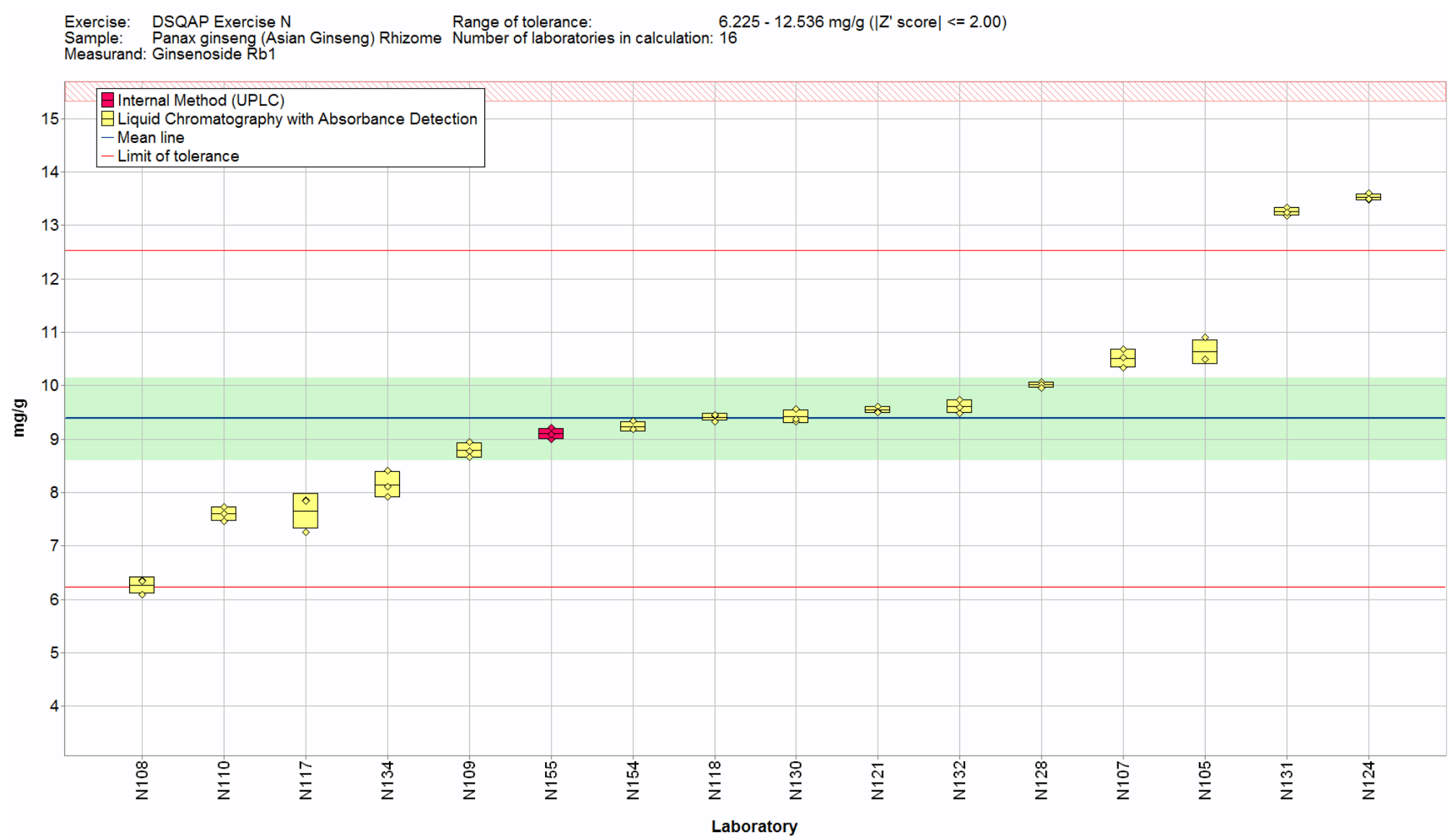

Figure 41. Ginsenoside Rb1 in candidate SRM 3384 Ground Asian Ginseng (Panax ginseng C.A. Meyer) Rhizome (data summary view - analytical method). In this view, individual laboratory data are plotted (diamonds) with the individual laboratory standard deviation (rectangle). The color of the data point represents the analytical method employed. The solid blue line represents the consensus mean, and the green shaded region represents the $95 \%$ confidence interval for the consensus mean. The solid red lines represent the consensus range of tolerance, calculated as the values above and below the consensus mean that result in an acceptable $Z_{\text {comm }}^{\prime}$ score, $\left|Z_{\text {comm }}^{\prime}\right| \leq 2$. The red shaded region (mostly off-chart) represents the NIST range of tolerance, which encompasses the NIST-determined value bounded by twice its standard deviation and represents the range that results in an acceptable $Z_{\text {NIST }}$ score, $\left|Z_{\mathrm{NIST}}\right| \leq 2$. 


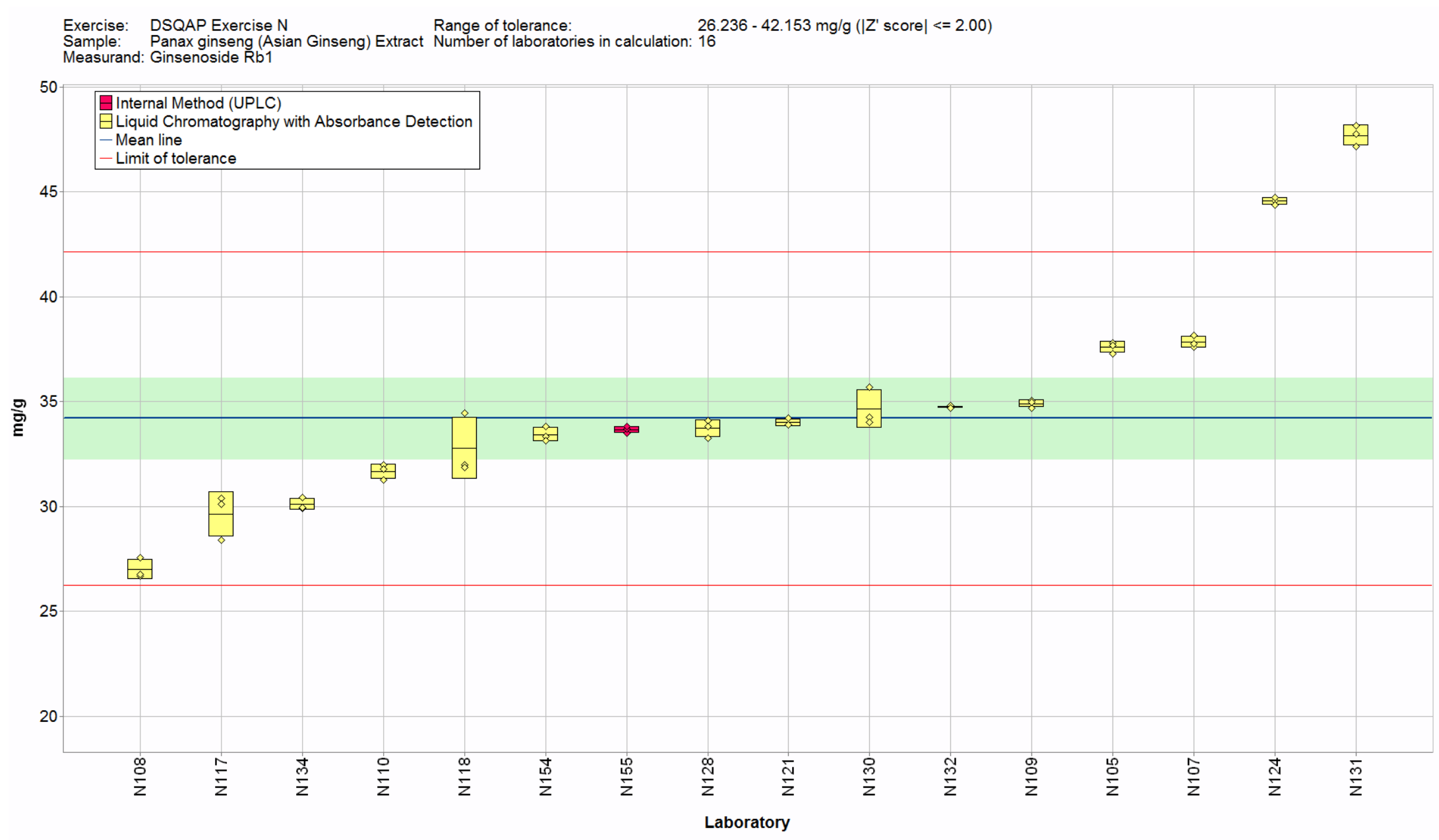

Figure 42. Ginsenoside Rb1 in candidate SRM 3385 Ground Asian Ginseng (Panax ginseng C.A. Meyer) Extract (data summary view - analytical method). In this view, individual laboratory data are plotted (diamonds) with the individual laboratory standard deviation (rectangle). The color of the data point represents the analytical method employed. The solid blue line represents the consensus mean and the green shaded region represents the $95 \%$ confidence interval for the consensus mean. The solid red lines represent the consensus range of tolerance, calculated as the values above and below the consensus mean that result in an acceptable $Z_{\text {comm }}^{\prime}$ score, $\left|Z_{\text {comm }}^{\prime}\right| \leq 2$. A NIST value has not been determined in this material. 


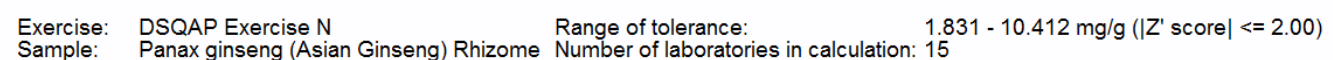
Sample: Panax ginseng (Asian Ginseng) Rhizome Number of laboratories in calculation: 15
Measurand: Ginsenoside Rb2

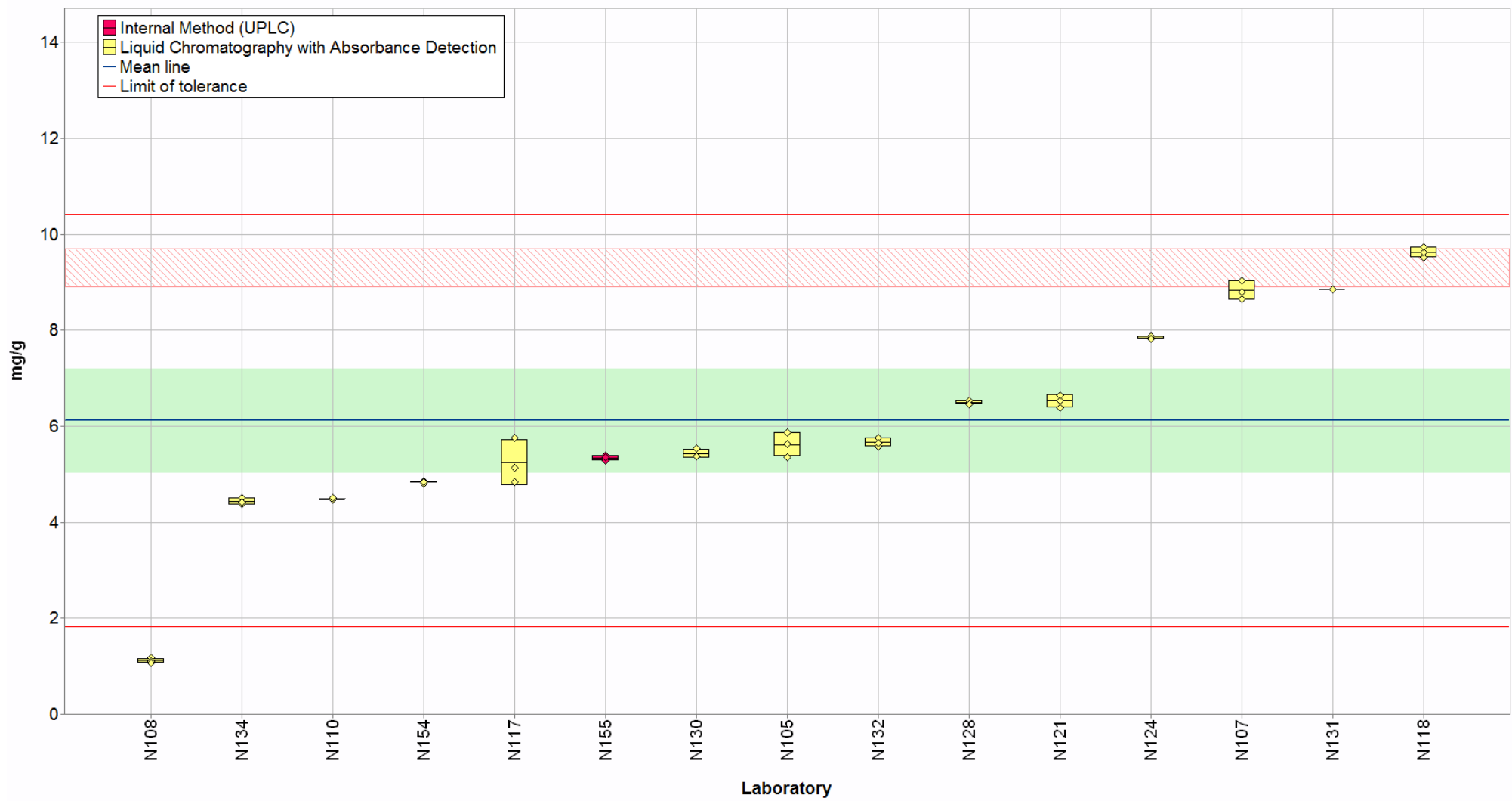

Figure 43. Ginsenoside Rb2 in candidate SRM 3384 Ground Asian Ginseng (Panax ginseng C.A. Meyer) Rhizome (data summary view - analytical method). In this view, individual laboratory data are plotted (diamonds) with the individual laboratory standard deviation (rectangle). The color of the data point represents the analytical method employed. The solid blue line represents the consensus mean, and the green shaded region represents the $95 \%$ confidence interval for the consensus mean. The solid red lines represent the consensus range of tolerance, calculated as the values above and below the consensus mean that result in an acceptable $Z_{\text {comm }}^{\prime}$ score, $\left|Z_{\text {comm }}^{\prime}\right| \leq 2$. The red shaded region represents the NIST range of tolerance, which encompasses the NIST-determined value bounded by twice its standard deviation and represents the range that results in an acceptable $Z_{\mathrm{NIST}}$ score, $\left|Z_{\text {NIST }}\right| \leq 2$. 


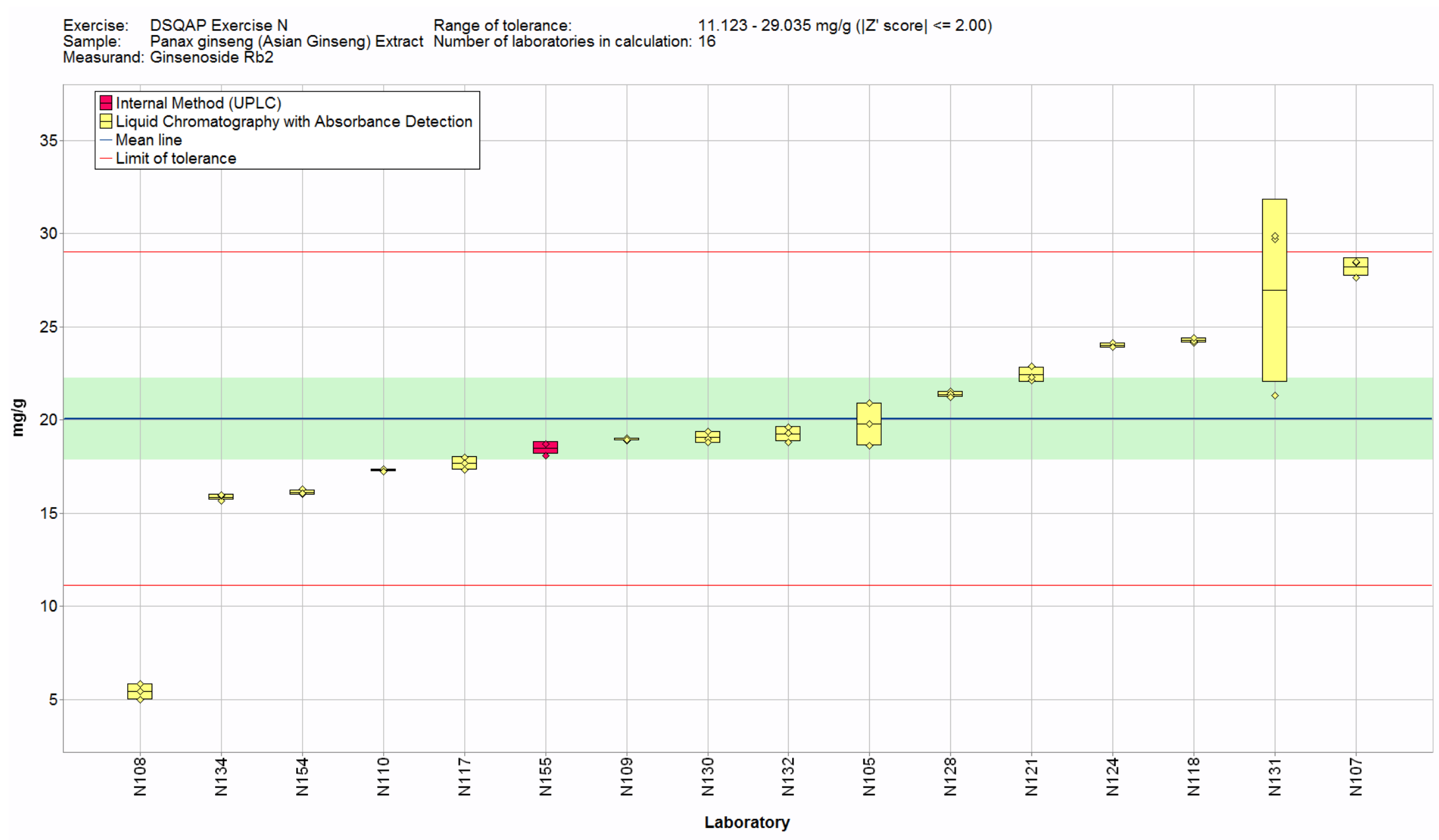

Figure 44. Ginsenoside Rb2 in candidate SRM 3385 Ground Asian Ginseng (Panax ginseng C.A. Meyer) Extract (data summary view - analytical method). In this view, individual laboratory data are plotted (diamonds) with the individual laboratory standard deviation (rectangle). The color of the data point represents the analytical method employed. The solid blue line represents the consensus mean and the green shaded region represents the $95 \%$ confidence interval for the consensus mean. The solid red lines represent the consensus range of tolerance, calculated as the values above and below the consensus mean that result in an acceptable $Z_{\text {comm }}^{\prime}$ score, $\left|Z_{\text {comm }}^{\prime}\right| \leq 2$. A NIST value has not been determined in this material. 


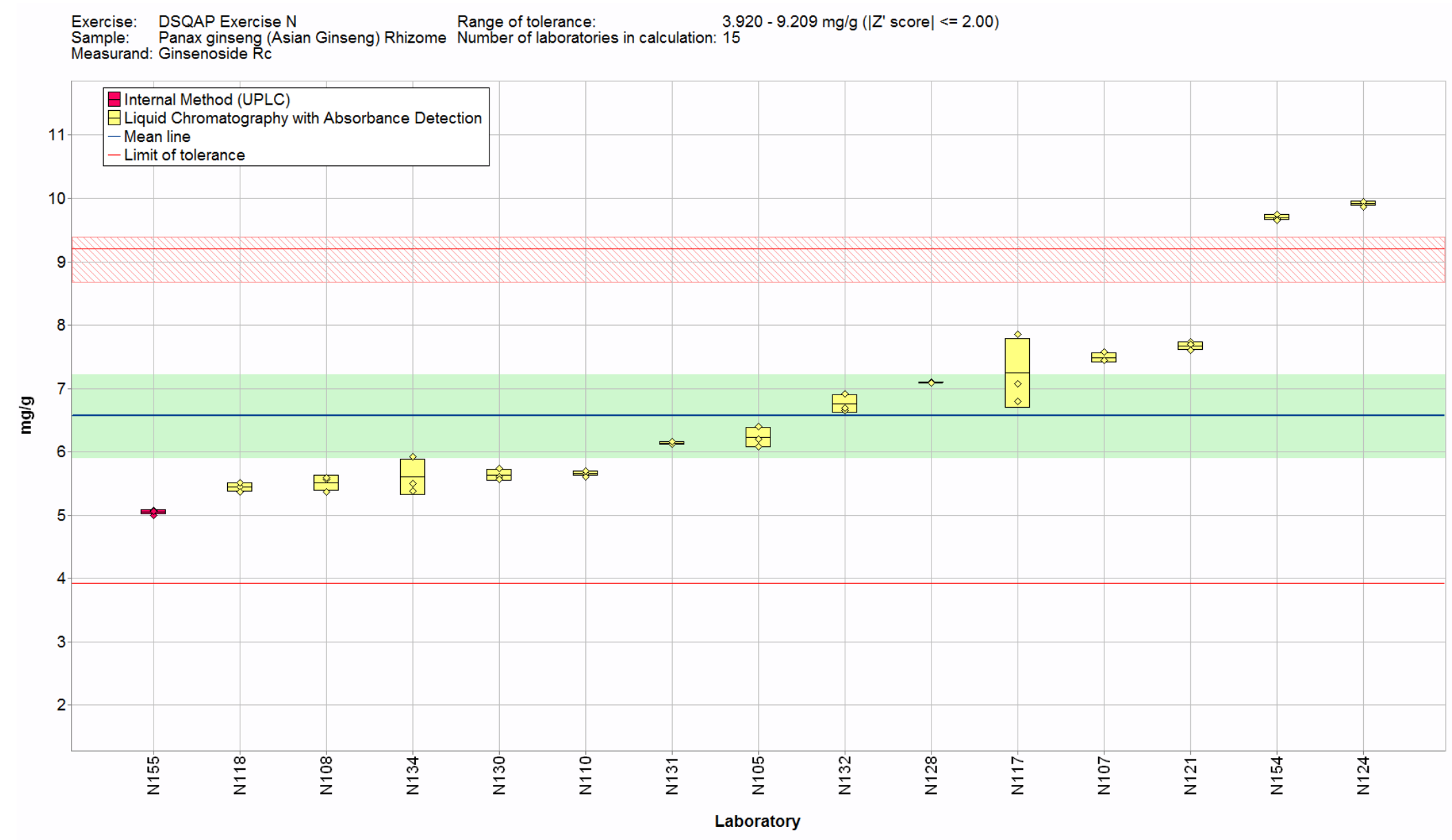

Figure 45. Ginsenoside Rc in candidate SRM 3384 Ground Asian Ginseng (Panax ginseng C.A. Meyer) Rhizome (data summary view - analytical method). In this view, individual laboratory data are plotted (diamonds) with the individual laboratory standard deviation (rectangle). The color of the data point represents the analytical method employed. The solid blue line represents the consensus mean and the green shaded region represents the $95 \%$ confidence interval for the consensus mean. The solid red lines represent the consensus range of tolerance, calculated as the values above and below the consensus mean that result in an acceptable $Z_{\text {comm }}^{\prime}$ score, $\left|Z_{\text {comm }}^{\prime}\right| \leq 2$. The red shaded region represents the NIST range of tolerance, which encompasses the NIST-determined value bounded by twice its standard deviation and represents the range that results in an acceptable $Z_{\mathrm{NIST}}$ score, $\left|Z_{\mathrm{NIST}}\right| \leq 2$. 


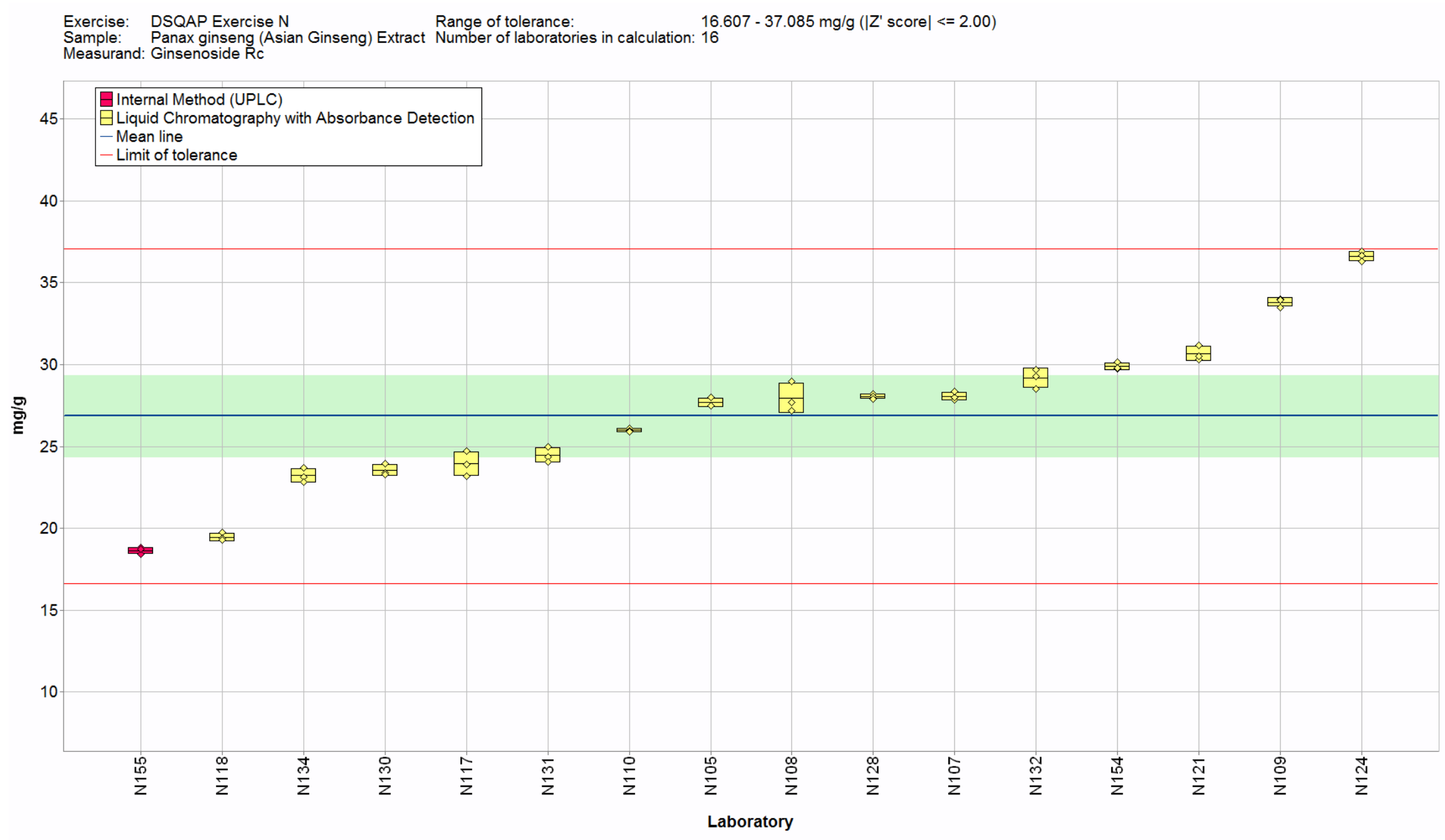

Figure 46. Ginsenoside Rc in candidate SRM 3385 Ground Asian Ginseng (Panax ginseng C.A. Meyer) Extract (data summary view - analytical method). In this view, individual laboratory data are plotted (diamonds) with the individual laboratory standard deviation (rectangle). The color of the data point represents the analytical method employed. The solid blue line represents the consensus mean and the green shaded region represents the $95 \%$ confidence interval for the consensus mean. The solid red lines represent the consensus range of tolerance, calculated as the values above and below the consensus mean that result in an acceptable $Z_{\text {comm }}^{\prime}$ score, $\left|Z_{\text {comm }}^{\prime}\right| \leq 2$. A NIST value has not been determined in this material. 


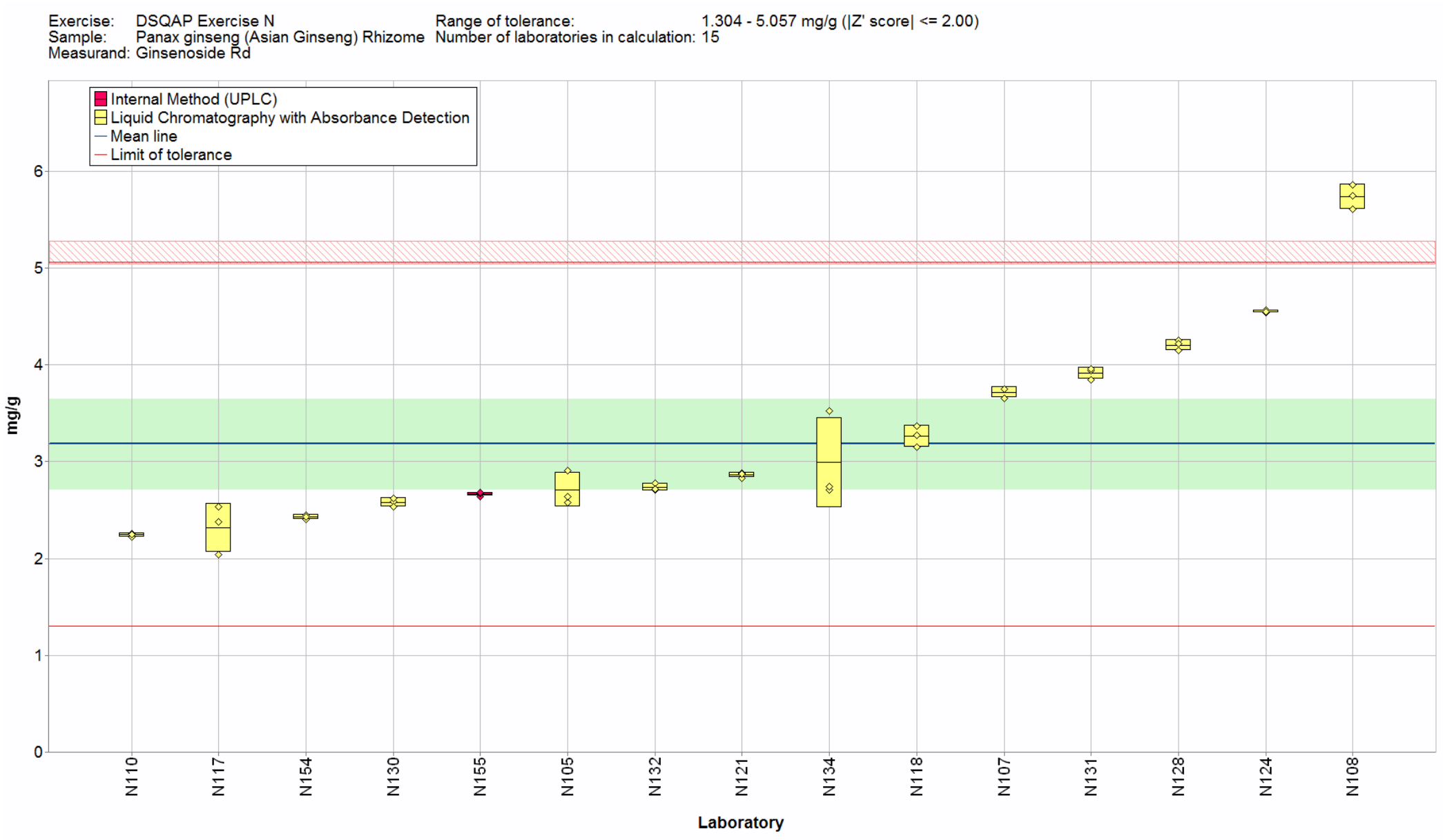

Figure 47. Ginsenoside Rd in candidate SRM 3384 Ground Asian Ginseng (Panax ginseng C.A. Meyer) Rhizome (data summary view - analytical method). In this view, individual laboratory data are plotted (diamonds) with the individual laboratory standard deviation (rectangle). The color of the data point represents the analytical method employed. The solid blue line represents the consensus mean and the green shaded region represents the $95 \%$ confidence interval for the consensus mean. The solid red lines represent the consensus range of tolerance, calculated as the values above and below the consensus mean that result in an acceptable $Z_{\text {comm }}^{\prime}$ score, $\left|Z_{\text {comm }}^{\prime}\right| \leq 2$. The red shaded region represents the NIST range of tolerance, which encompasses the NIST-determined value bounded by twice its standard deviation and represents the range that results in an acceptable $Z_{\mathrm{NIST}}$ score, $\left|Z_{\mathrm{NIST}}\right| \leq 2$. 


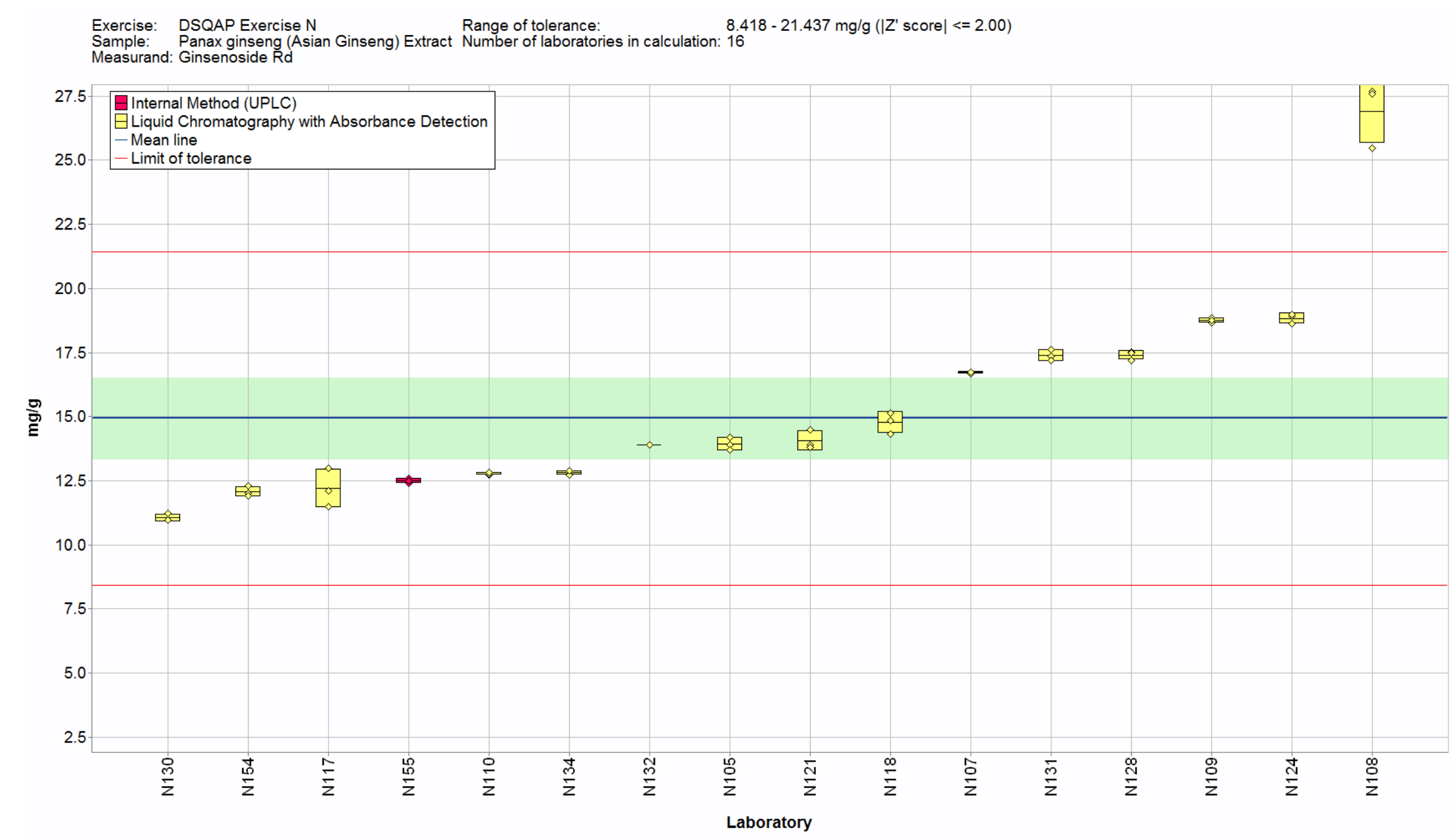

Figure 48. Ginsenoside Rd in candidate SRM 3385 Ground Asian Ginseng (Panax ginseng C.A. Meyer) Extract (data summary view - analytical method). In this view, individual laboratory data are plotted (diamonds) with the individual laboratory standard deviation (rectangle). The color of the data point represents the analytical method employed. The solid blue line represents the consensus mean and the green shaded region represents the $95 \%$ confidence interval for the consensus mean. The solid red lines represent the consensus range of tolerance, calculated as the values above and below the consensus mean that result in an acceptable $Z_{\text {comm }}^{\prime}$ score, $\left|Z_{\text {comm }}^{\prime}\right| \leq 2$. A NIST value has not been determined in this material. 


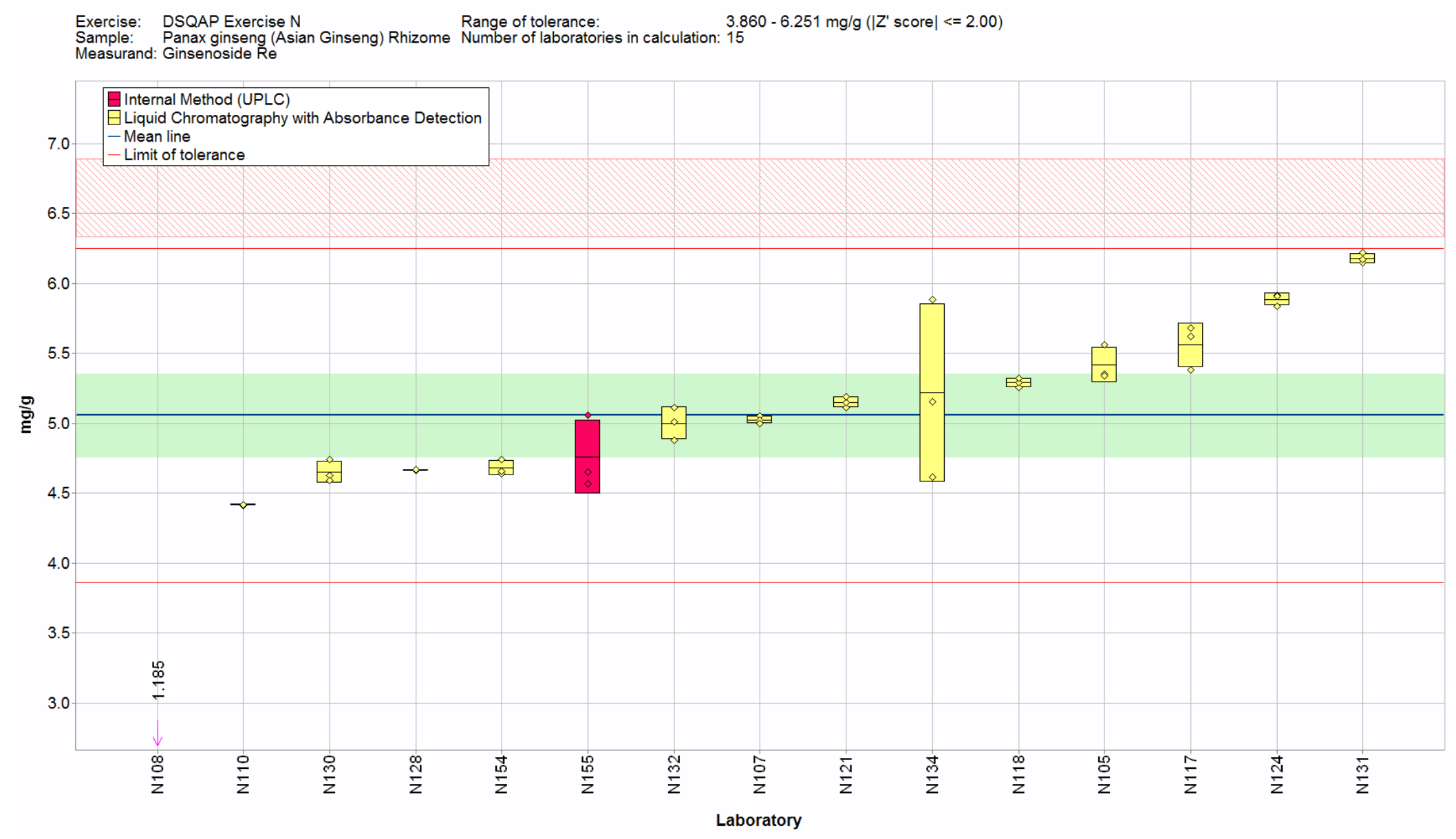

Figure 49. Ginsenoside Re in candidate SRM 3384 Ground Asian Ginseng (Panax ginseng C.A. Meyer) Rhizome (data summary view - analytical method). In this view, individual laboratory data are plotted (diamonds) with the individual laboratory standard deviation (rectangle). The color of the data point represents the analytical method employed. The solid blue line represents the consensus mean and the green shaded region represents the $95 \%$ confidence interval for the consensus mean. The solid red lines represent the consensus range of tolerance, calculated as the values above and below the consensus mean that result in an acceptable $Z_{\text {comm }}^{\prime}$ score, $\left|Z_{\text {comm }}^{\prime}\right| \leq 2$. The red shaded region represents the NIST range of tolerance, which encompasses the NIST-determined value bounded by twice its standard deviation and represents the range that results in an acceptable $Z_{\mathrm{NIST}}$ score, $\left|Z_{\mathrm{NIST}}\right| \leq 2$. 


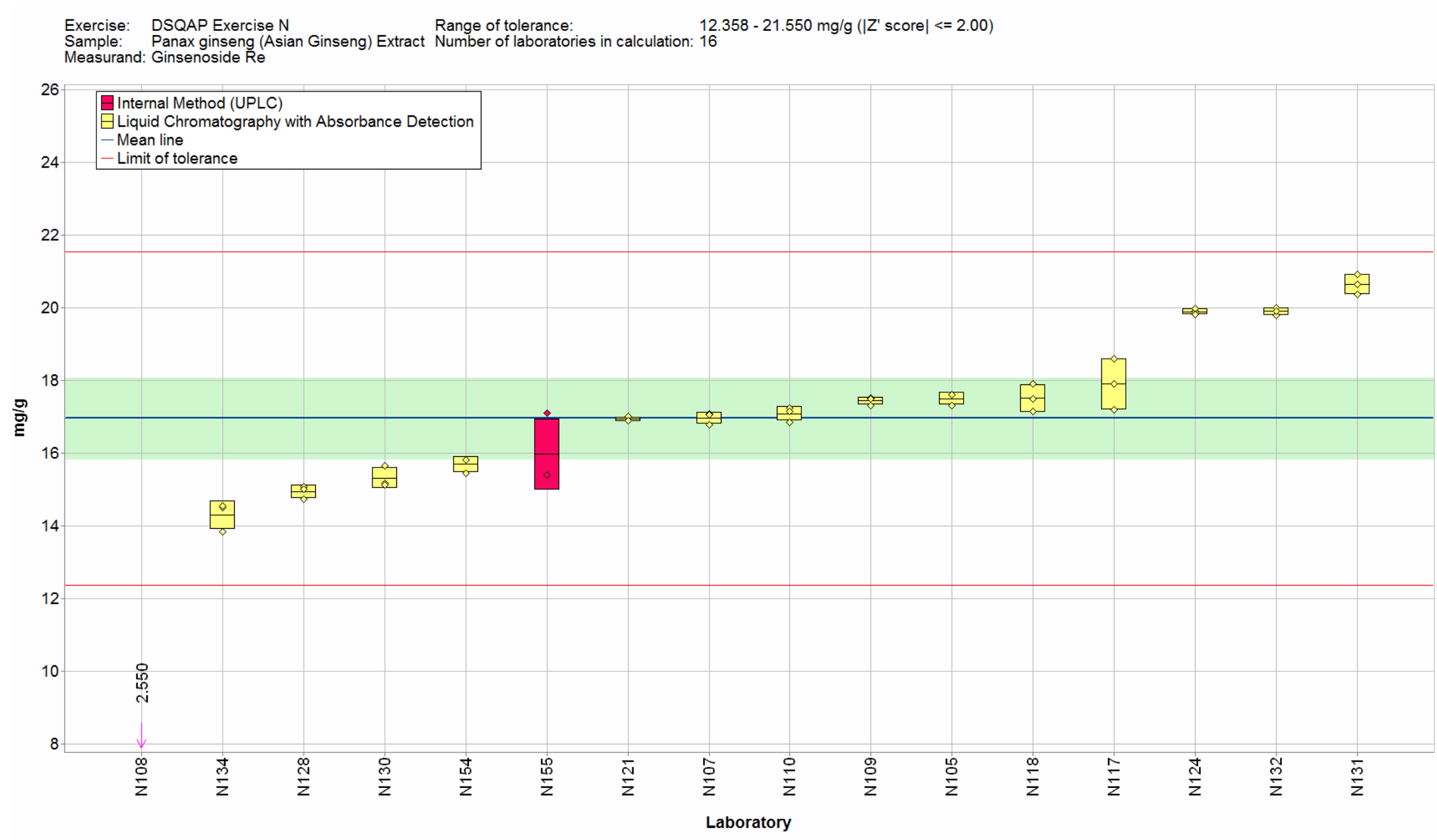

Figure 50. Ginsenoside Re in candidate SRM 3385 Ground Asian Ginseng (Panax ginseng C.A. Meyer) Extract (data summary view - analytical method). In this view, individual laboratory data are plotted (diamonds) with the individual laboratory standard deviation (rectangle). The color of the data point represents the analytical method employed. The solid blue line represents the consensus mean and the green shaded region represents the $95 \%$ confidence interval for the consensus mean. The solid red lines represent the consensus range of tolerance, calculated as the values above and below the consensus mean that result in an acceptable $Z_{\text {comm }}^{\prime}$ score, $\left|Z_{\text {comm }}^{\prime}\right| \leq 2$. A NIST value has not been determined in this material. 


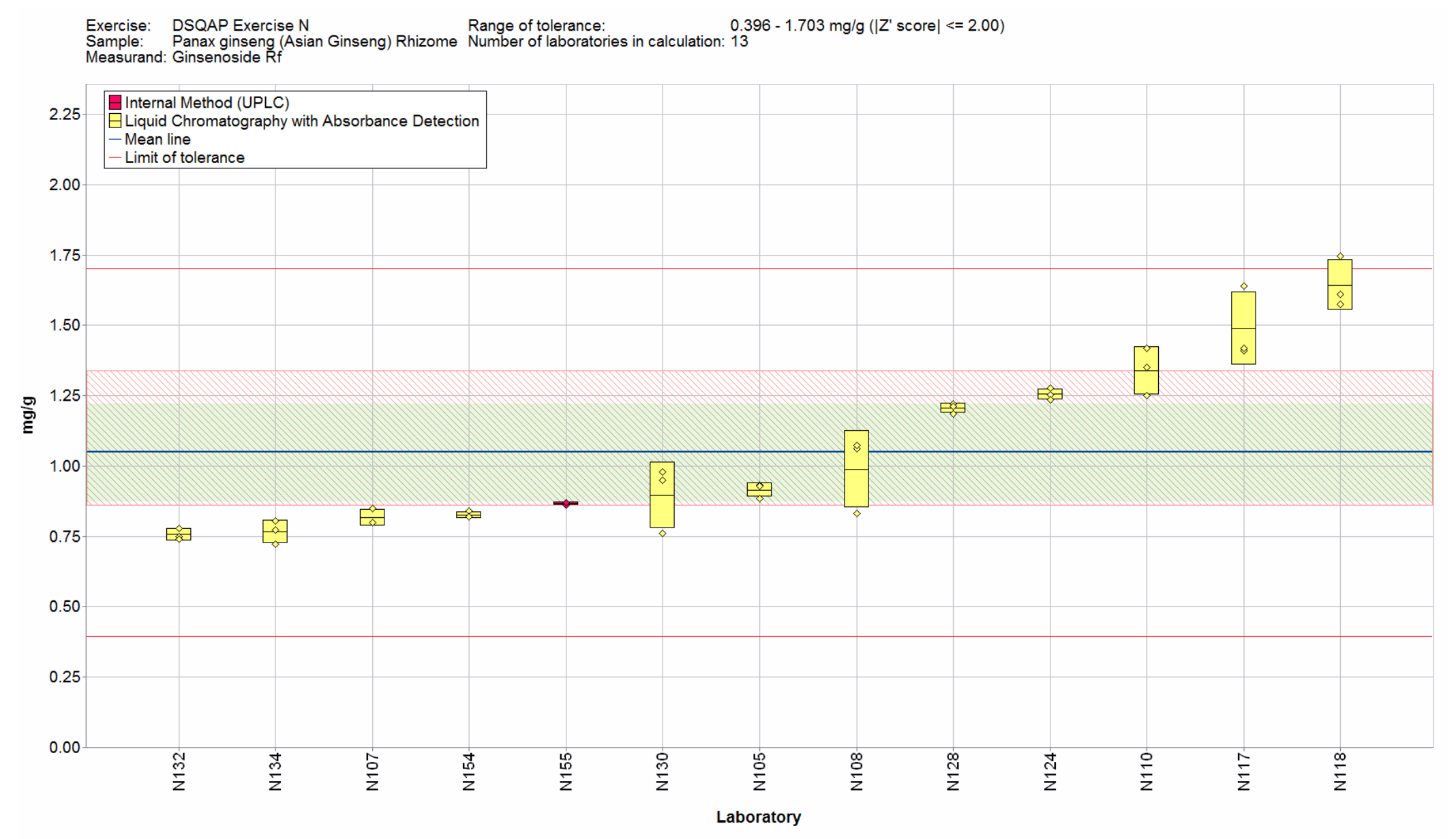

Figure 51. Ginsenoside Rf in candidate SRM 3384 Ground Asian Ginseng (Panax ginseng C.A. Meyer) Rhizome (data summary view - analytical method). In this view, individual laboratory data are plotted (diamonds) with the individual laboratory standard deviation (rectangle). The color of the data point represents the analytical method employed. The solid blue line represents the consensus mean and the green shaded region represents the $95 \%$ confidence interval for the consensus mean. The solid red lines represent the consensus range of tolerance, calculated as the values above and below the consensus mean that result in an acceptable $Z_{\text {comm }}^{\prime}$ score, $\left|Z_{\text {comm }}^{\prime}\right| \leq 2$. The red shaded region represents the NIST range of tolerance, which encompasses the NIST-determined value bounded by twice its standard deviation and represents the range that results in an acceptable $Z_{\mathrm{NIST}}$ score, $\left|Z_{\mathrm{NIST}}\right| \leq 2$. 

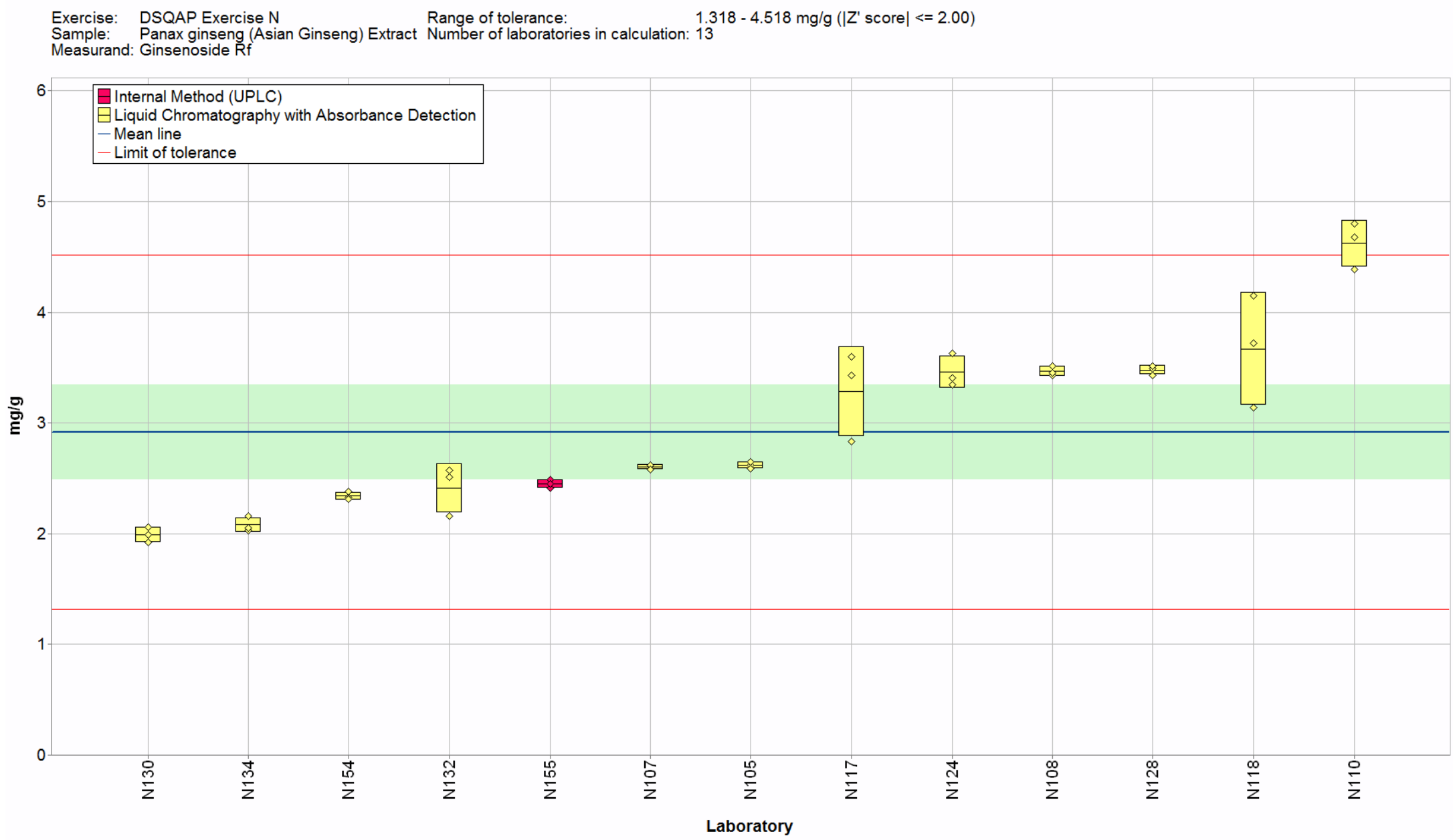

Figure 52. Ginsenoside Rf in candidate SRM 3385 Ground Asian Ginseng (Panax ginseng C.A. Meyer) Extract (data summary view - analytical method). In this view, individual laboratory data are plotted (diamonds) with the individual laboratory standard deviation (rectangle). The color of the data point represents the analytical method employed. The solid blue line represents the consensus mean and the green shaded region represents the $95 \%$ confidence interval for the consensus mean. The solid red lines represent the consensus range of tolerance, calculated as the values above and below the consensus mean that result in an acceptable $Z_{\text {comm }}^{\prime}$ score, $\left|Z_{\text {comm }}^{\prime}\right| \leq 2$. A NIST value has not been determined in this material. 


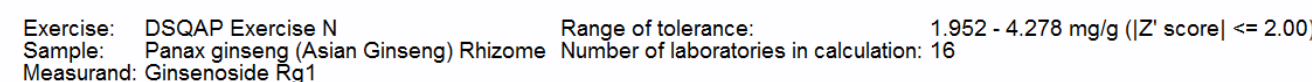

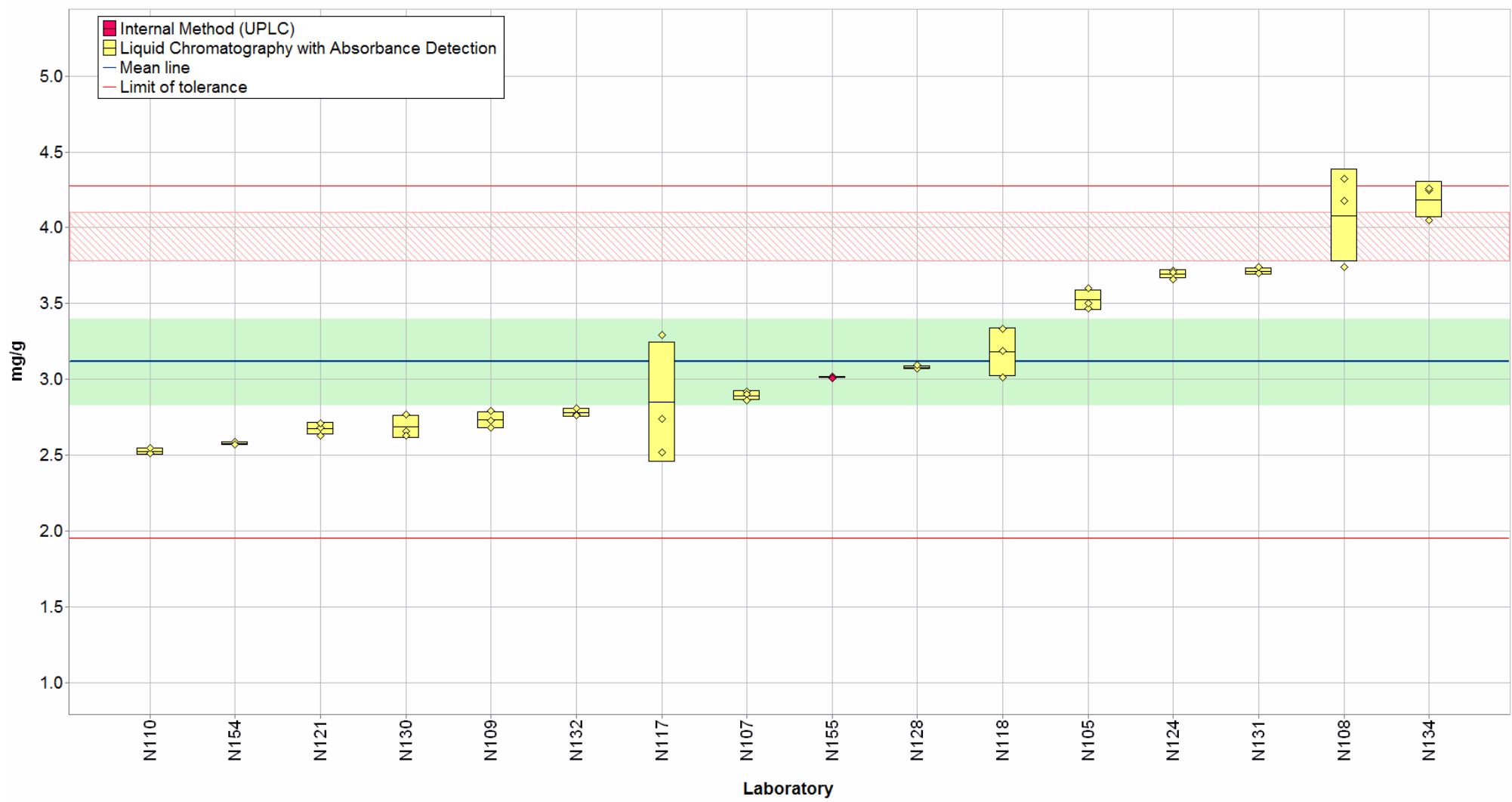

Figure 53. Ginsenoside Rg1 in candidate SRM 3384 Ground Asian Ginseng (Panax ginseng C.A. Meyer) Rhizome (data summary view - analytical method). In this view, individual laboratory data are plotted (diamonds) with the individual laboratory standard deviation (rectangle). The color of the data point represents the analytical method employed. The solid blue line represents the consensus mean and the green shaded region represents the $95 \%$ confidence interval for the consensus mean. The solid red lines represent the consensus range of tolerance, calculated as the values above and below the consensus mean that result in an acceptable $Z_{\text {comm }}^{\prime}$ score, $\left|Z_{\text {comm }}^{\prime}\right| \leq 2$. The red shaded region represents the NIST range of tolerance, which encompasses the NIST-determined value bounded by twice its standard deviation and represents the range that results in an acceptable $Z_{\mathrm{NIST}}$ score, $\left|Z_{\text {NIST }}\right| \leq 2$. 


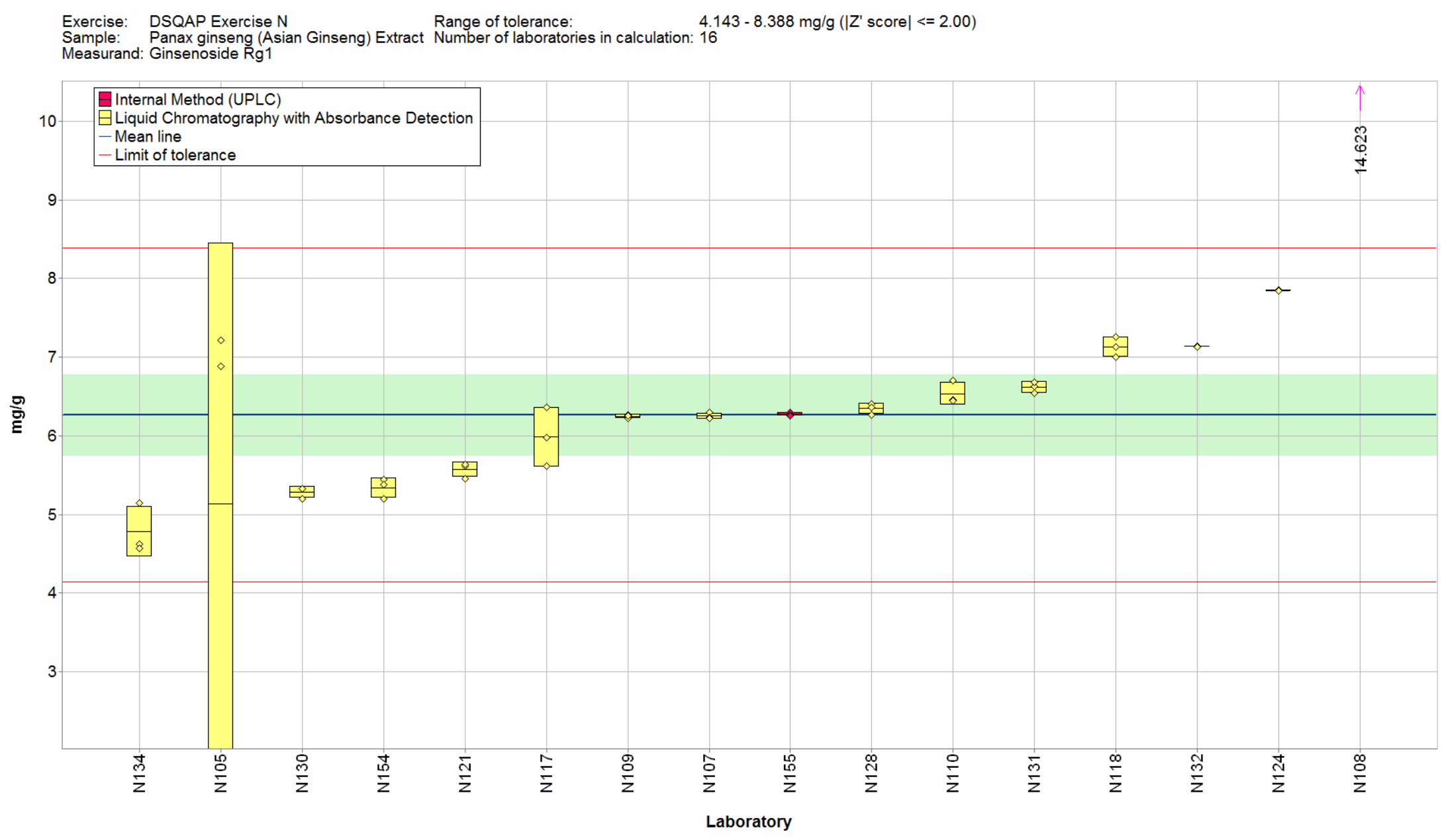

Figure 54. Ginsenoside Rg1 in SRM 3385 Ground Asian Ginseng (Panax ginseng C.A. Meyer) Extract (data summary view - analytical method). In this view, individual laboratory data are plotted (diamonds) with the individual laboratory standard deviation (rectangle). The color of the data point represents the analytical method employed. The solid blue line represents the consensus mean and the green shaded region represents the $95 \%$ confidence interval for the consensus mean. The solid red lines represent the consensus range of tolerance, calculated as the values above and below the consensus mean that result in an acceptable $Z_{\text {comm }}^{\prime}$ score, $\left|Z_{\text {comm }}^{\prime}\right| \leq 2$. A NIST value has not been determined in this material. 


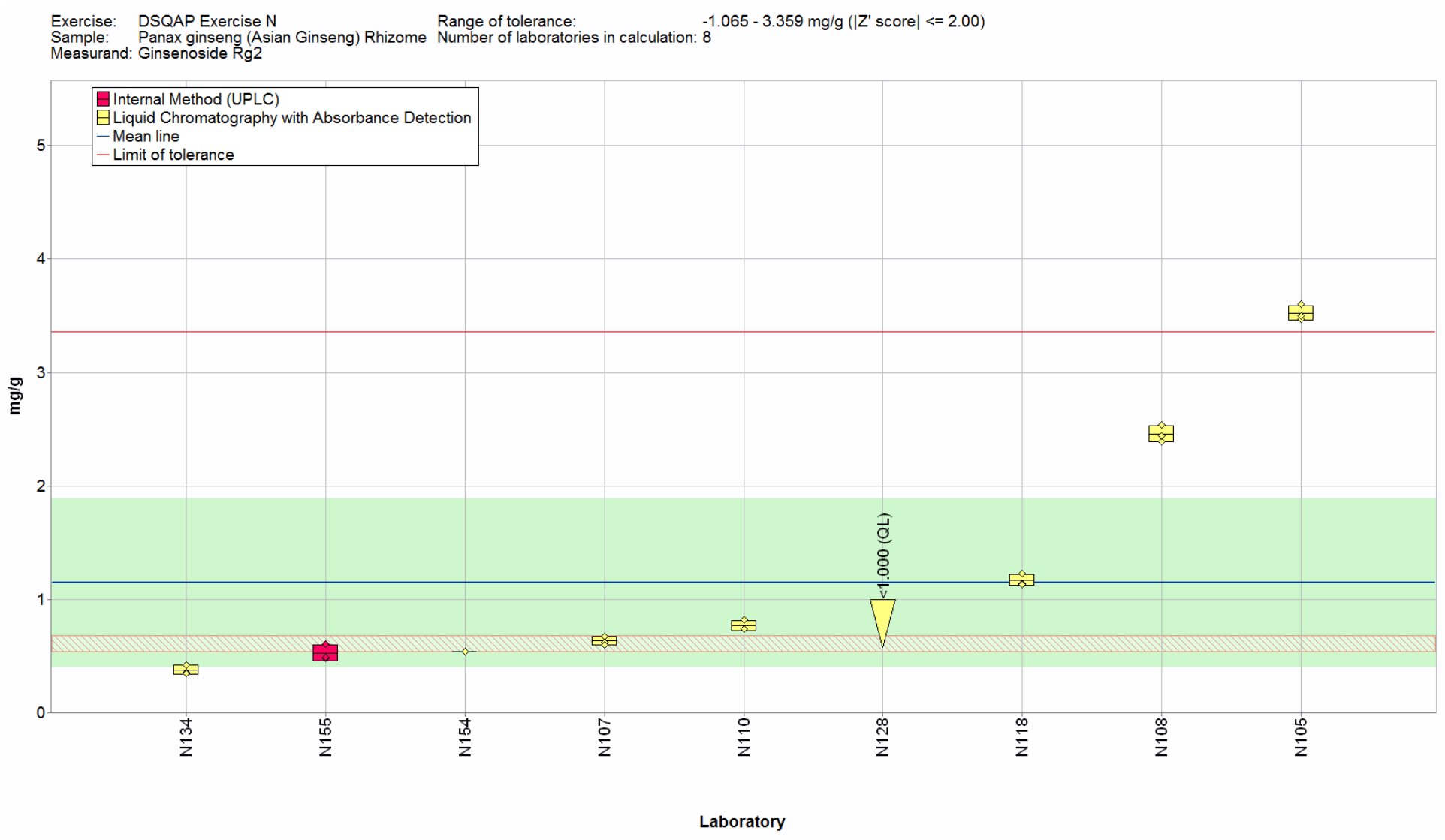

Figure 55. Ginsenoside Rg2 in candidate SRM 3384 Ground Asian Ginseng (Panax ginseng C.A. Meyer) Rhizome (data summary view - analytical method). In this view, individual laboratory data are plotted (diamonds) with the individual laboratory standard deviation (rectangle). The color of the data point represents the analytical method employed. Laboratory data shown as a triangle indicates that a "less than" result was submitted, and the base of the triangle is displayed at the reported laboratory detection limit. The solid blue line represents the consensus mean and the green shaded region represents the $95 \%$ confidence interval for the consensus mean. The solid red line represents the upper consensus range of tolerance, calculated as the values above and below the consensus mean that result in an acceptable $Z_{\text {comm }}^{\prime}$ score, $\left|Z_{\text {comm }}^{\prime}\right| \leq 2$, with the lower range set at zero. The red shaded region represents the NIST range of tolerance, which encompasses the NIST-determined value bounded by twice its standard deviation and represents the range that results in an acceptable $Z_{\text {NIST }}$ score, $\left|Z_{\text {NIST }}\right| \leq 2$. 


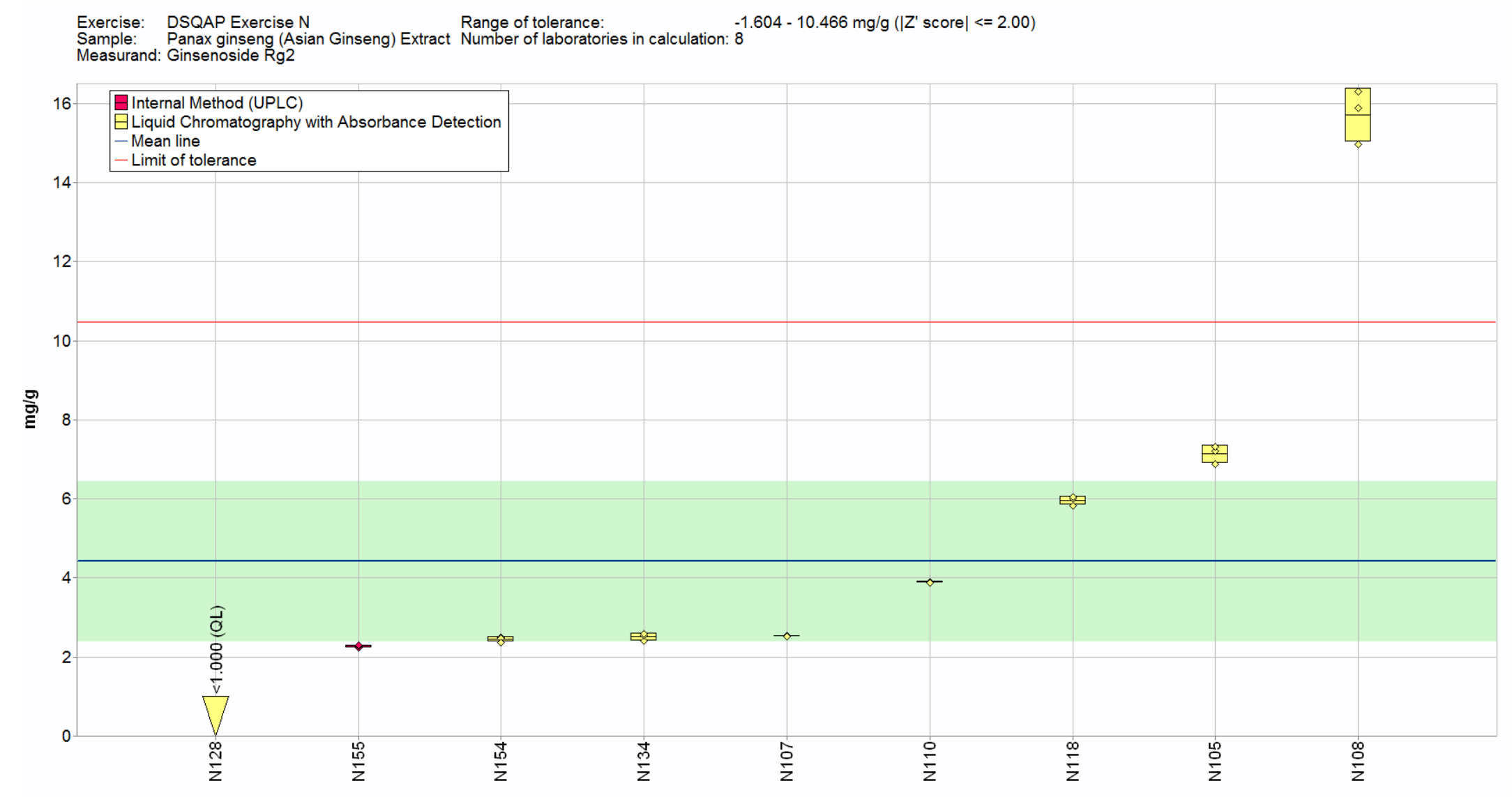

Laboratory

Figure 56. Ginsenoside Rg2 in candidate SRM 3385 Ground Asian Ginseng (Panax ginseng C.A. Meyer) Extract (data summary view - analytical method). In this view, individual laboratory data are plotted (diamonds) with the individual laboratory standard deviation (rectangle). The color of the data point represents the analytical method employed. Laboratory data shown as a triangle indicates that a "less than" result was submitted, and the base of the triangle is displayed at the reported laboratory detection limit. The solid blue line represents the consensus mean and the green shaded region represents the $95 \%$ confidence interval for the consensus mean. The solid red line represents the upper consensus range of tolerance, calculated as the values above and below the consensus mean that result in an acceptable $Z_{\text {comm }}^{\prime}$ score, $\left|Z_{\text {comm }}^{\prime}\right| \leq 2$, with the lower range set at zero. A NIST value has not been determined in this material. 
DSQAP Exercise N, Measurand: Ginsenoside Rb1

No. of laboratories: 16

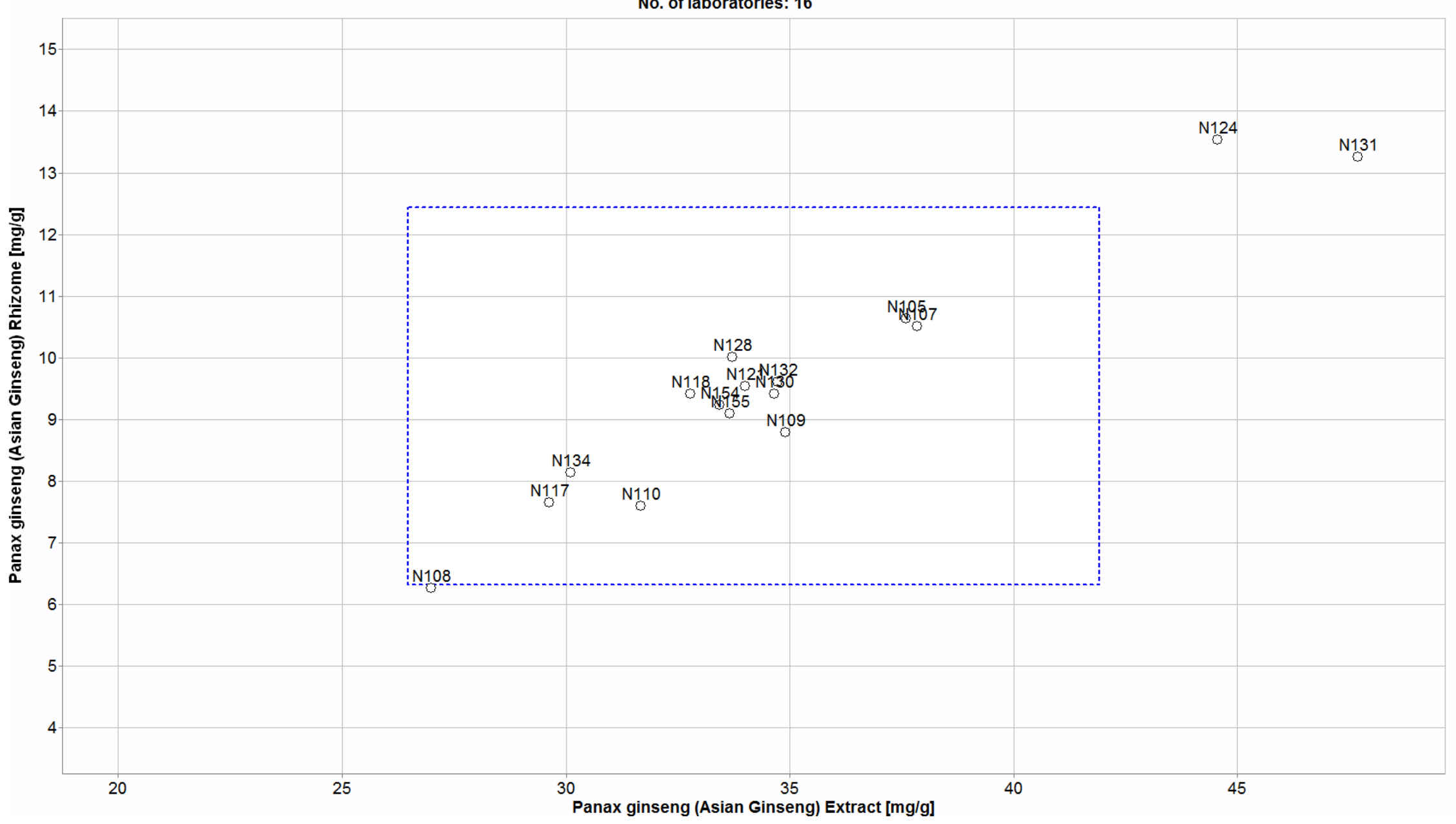

Figure 57. Laboratory means for ginsenoside Rb1 in Asian ginseng rhizome and Asian ginseng extract (sample/sample comparison view). In this view, the individual laboratory mean for one sample (rhizome) is compared to the mean for a second sample (extract). The dotted blue box represents the consensus range of tolerance for Asian ginseng extract (x-axis) and Asian ginseng rhizome ( $\mathrm{y}$-axis), calculated as the values above and below the consensus means that result in an acceptable $Z_{\text {comm }}^{\prime}$ score, $\left|Z_{\text {comm }}^{\prime}\right| \leq 2$. 
DSQAP Exercise N, Measurand: Ginsenoside Rb2

No. of laboratories: 15

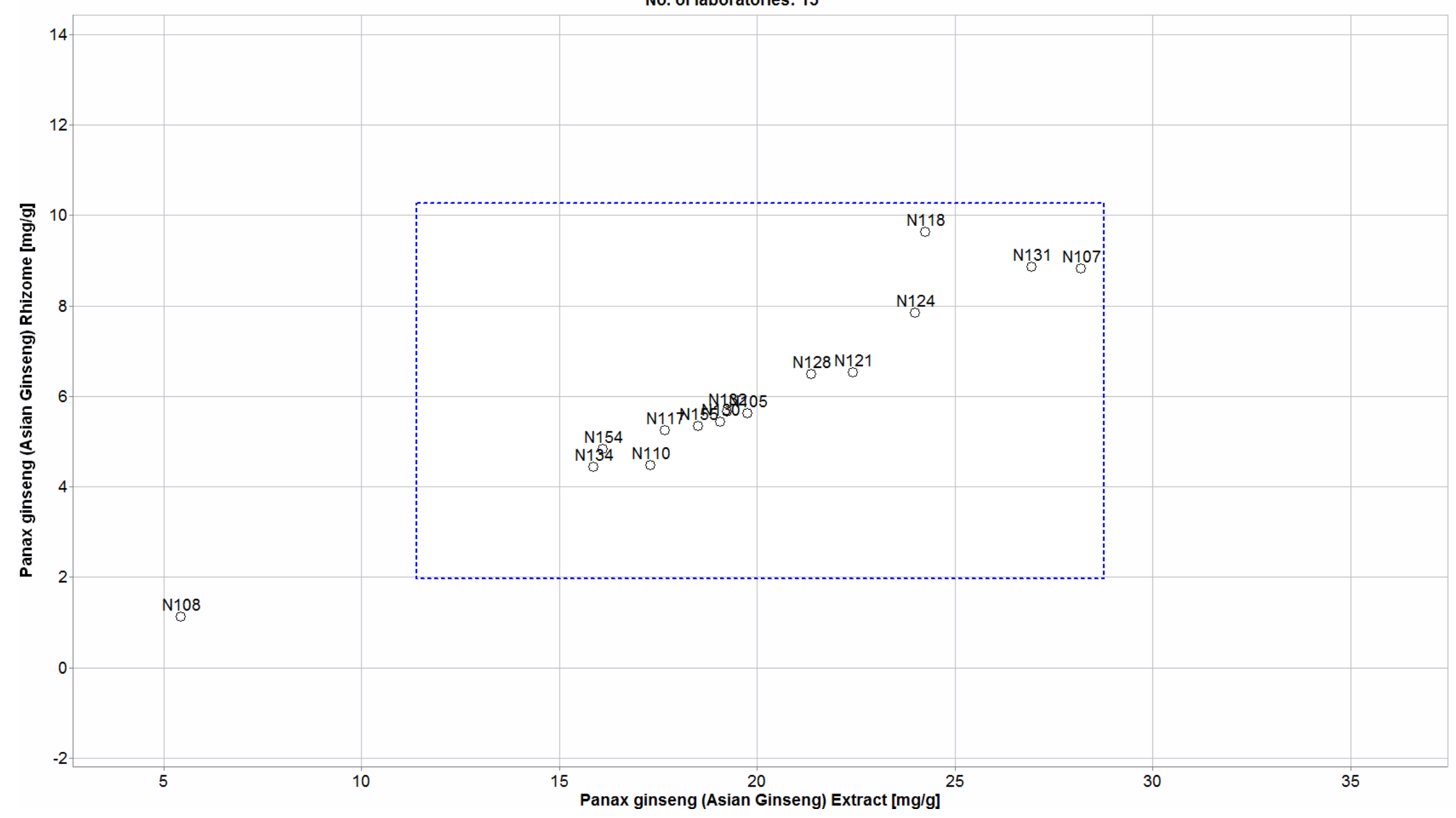

Figure 58. Laboratory means for ginsenoside $\mathrm{Rb} 2$ in Asian ginseng rhizome and Asian ginseng extract (sample/sample comparison view). In this view, the individual laboratory mean for one sample (rhizome) is compared to the mean for a second sample (extract). The dotted blue box represents the consensus range of tolerance for Asian ginseng extract (x-axis) and Asian ginseng rhizome ( $y$-axis), calculated as the values above and below the consensus means that result in an acceptable $Z_{\text {comm }}^{\prime}$ score, $\left|Z_{\text {comm }}^{\prime}\right| \leq 2$. 


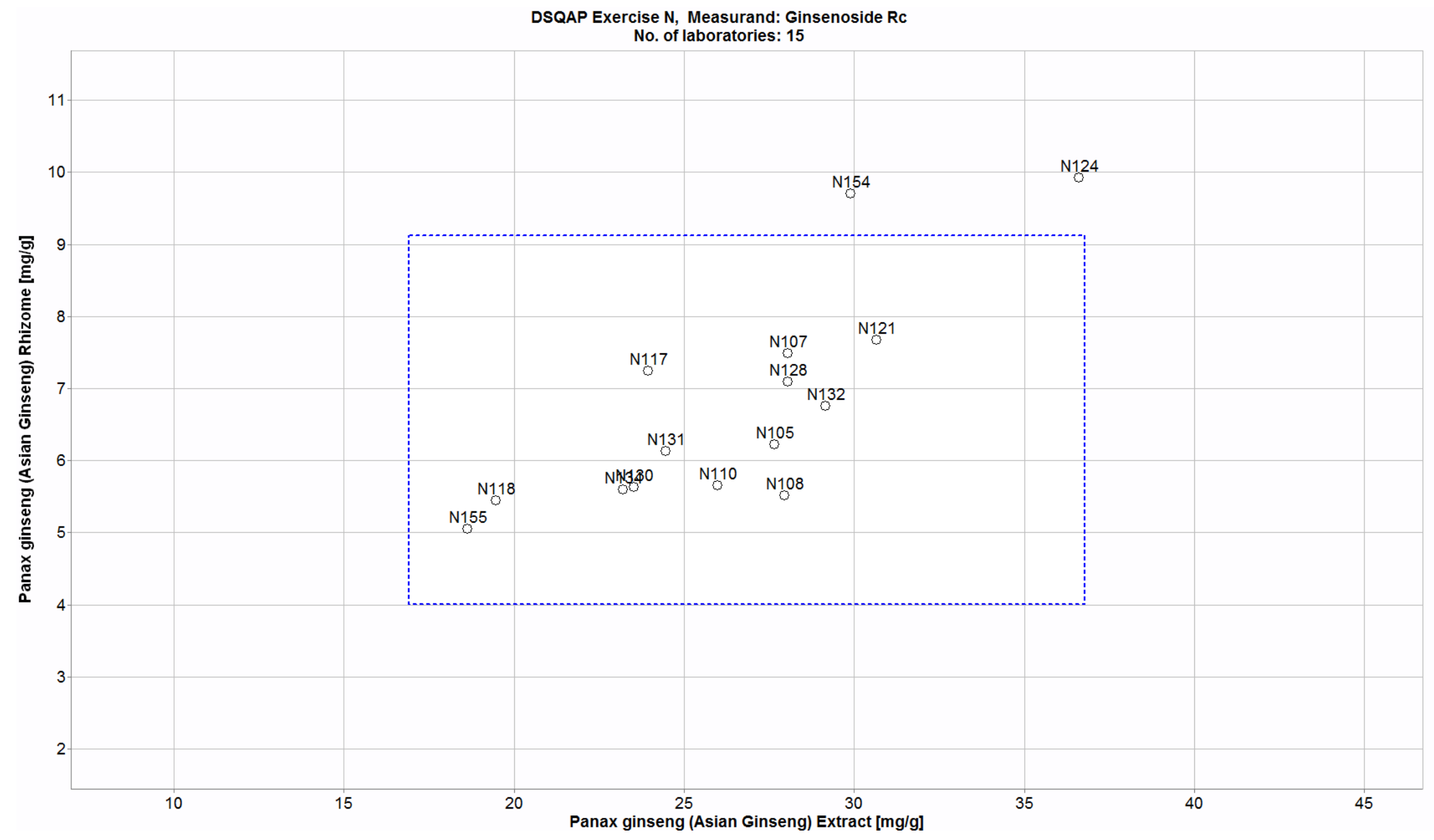

Figure 59. Laboratory means for ginsenoside Rc in Asian ginseng rhizome and Asian ginseng extract (sample/sample comparison view). In this view, the individual laboratory mean for one sample (rhizome) is compared to the mean for a second sample (extract). The dotted blue box represents the consensus range of tolerance for Asian ginseng extract (x-axis) and Asian ginseng rhizome ( $\mathrm{y}$-axis), calculated as the values above and below the consensus means that result in an acceptable $Z_{\text {comm }}^{\prime}$ score, $\left|Z_{\text {comm }}^{\prime}\right| \leq 2$. 


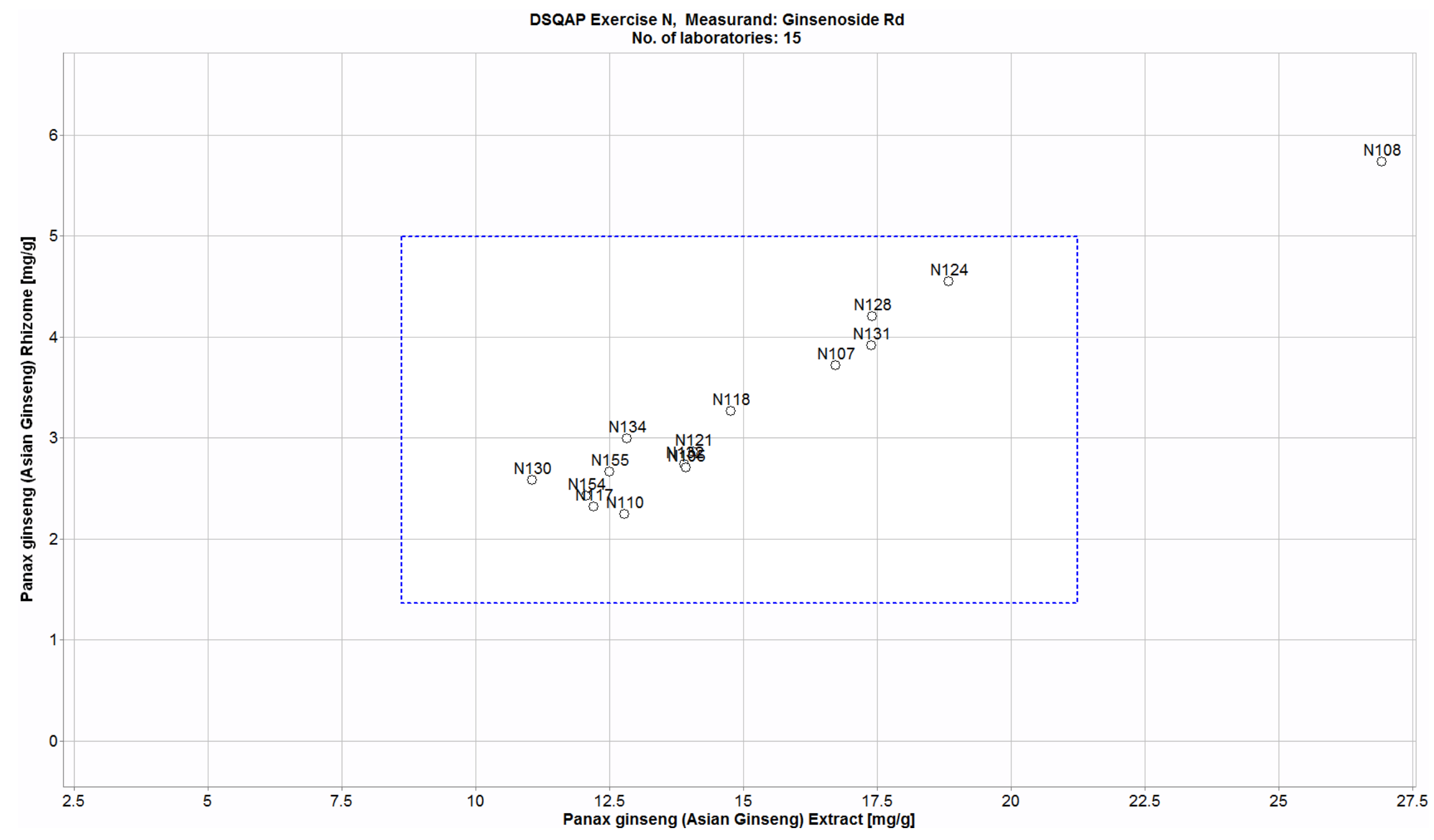

Figure 60. Laboratory means for ginsenoside Rd in Asian ginseng rhizome and Asian ginseng extract (sample/sample comparison view). In this view, the individual laboratory mean for one sample (rhizome) is compared to the mean for a second sample (extract). The dotted blue box represents the consensus range of tolerance for Asian ginseng extract (x-axis) and Asian ginseng rhizome ( $\mathrm{y}$-axis), calculated as the values above and below the consensus means that result in an acceptable $Z_{\text {comm }}^{\prime}$ score, $\left|Z_{\text {comm }}^{\prime}\right| \leq 2$. 


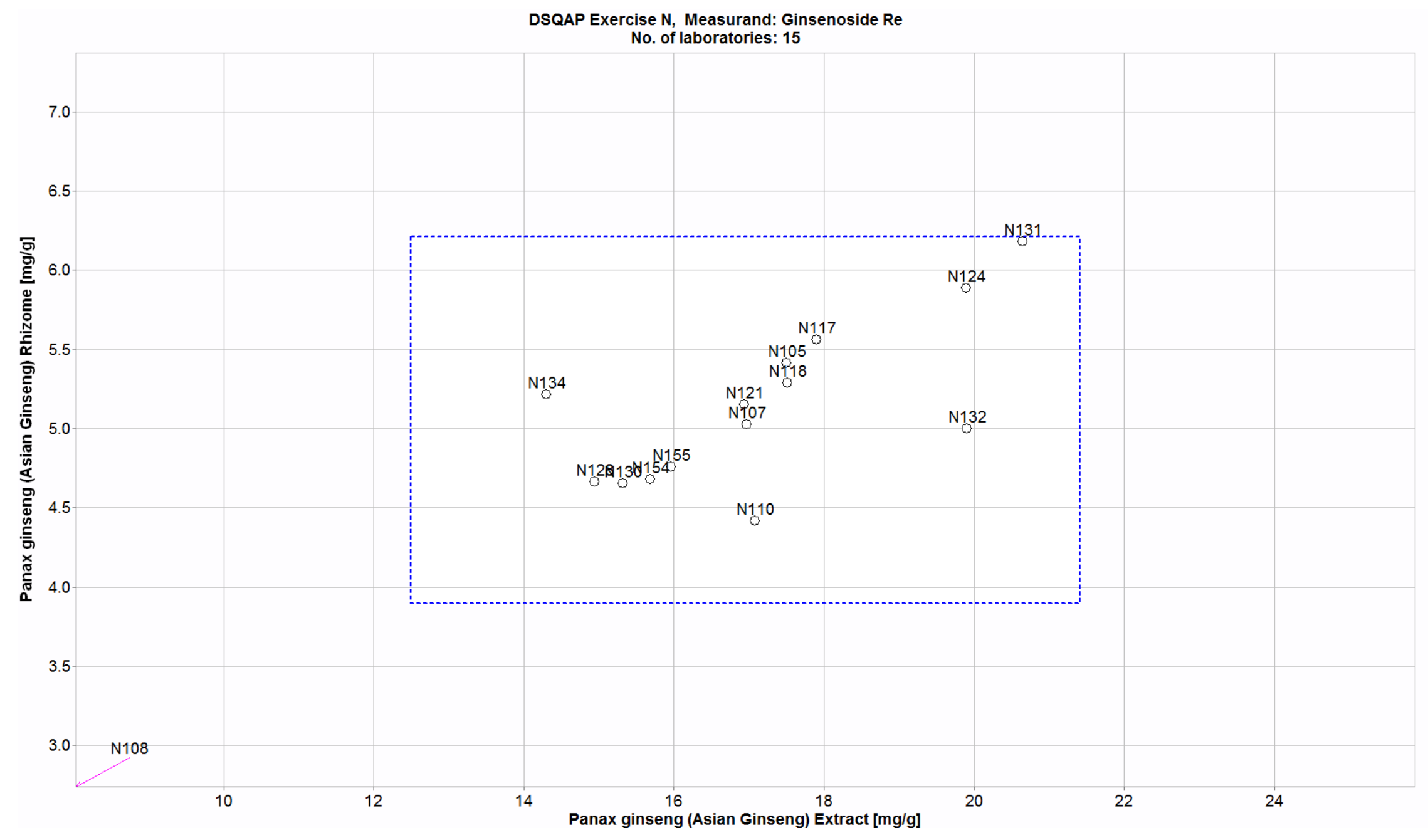

Figure 61. Laboratory means for ginsenoside Re in Asian ginseng rhizome and Asian ginseng extract (sample/sample comparison view). In this view, the individual laboratory mean for one sample (rhizome) is compared to the mean for a second sample (extract). The dotted blue box represents the consensus range of tolerance for Asian ginseng extract (x-axis) and Asian ginseng rhizome ( $\mathrm{y}$-axis), calculated as the values above and below the consensus means that result in an acceptable $Z_{\text {comm }}^{\prime}$ score, $\left|Z_{\text {comm }}^{\prime}\right| \leq 2$. 


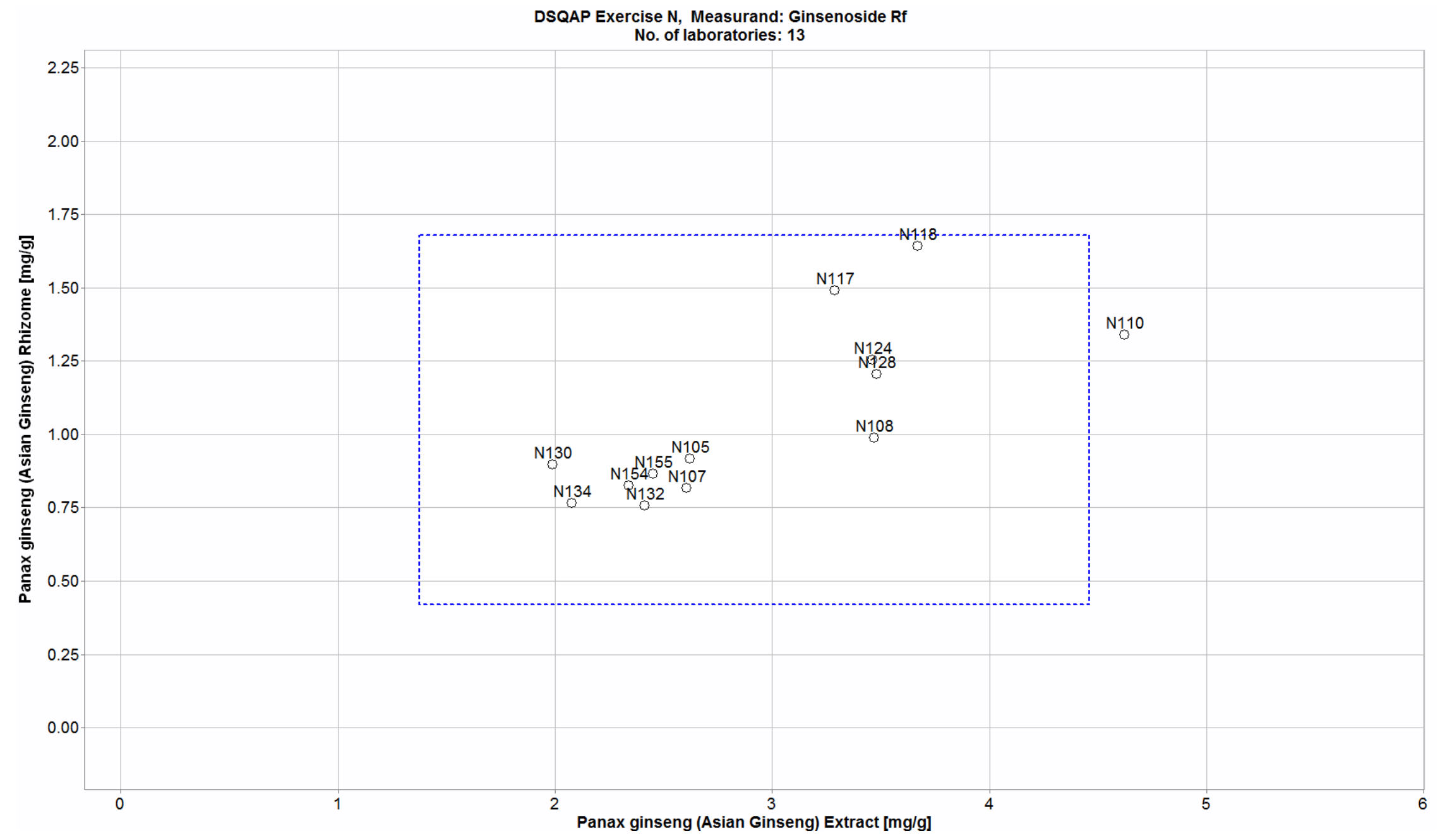

Figure 62. Laboratory means for ginsenoside Rf in Asian ginseng rhizome and Asian ginseng extract (sample/sample comparison view). In this view, the individual laboratory mean for one sample (rhizome) is compared to the mean for a second sample (extract). The dotted blue box represents the consensus range of tolerance for Asian ginseng extract (x-axis) and Asian ginseng rhizome ( $y$-axis), calculated as the values above and below the consensus means that result in an acceptable $Z_{\text {comm }}^{\prime}$ score, $\left|Z_{\text {comm }}^{\prime}\right| \leq 2$. 
DSQAP Exercise N, Measurand: Ginsenoside Rg1

No. of laboratories: 16

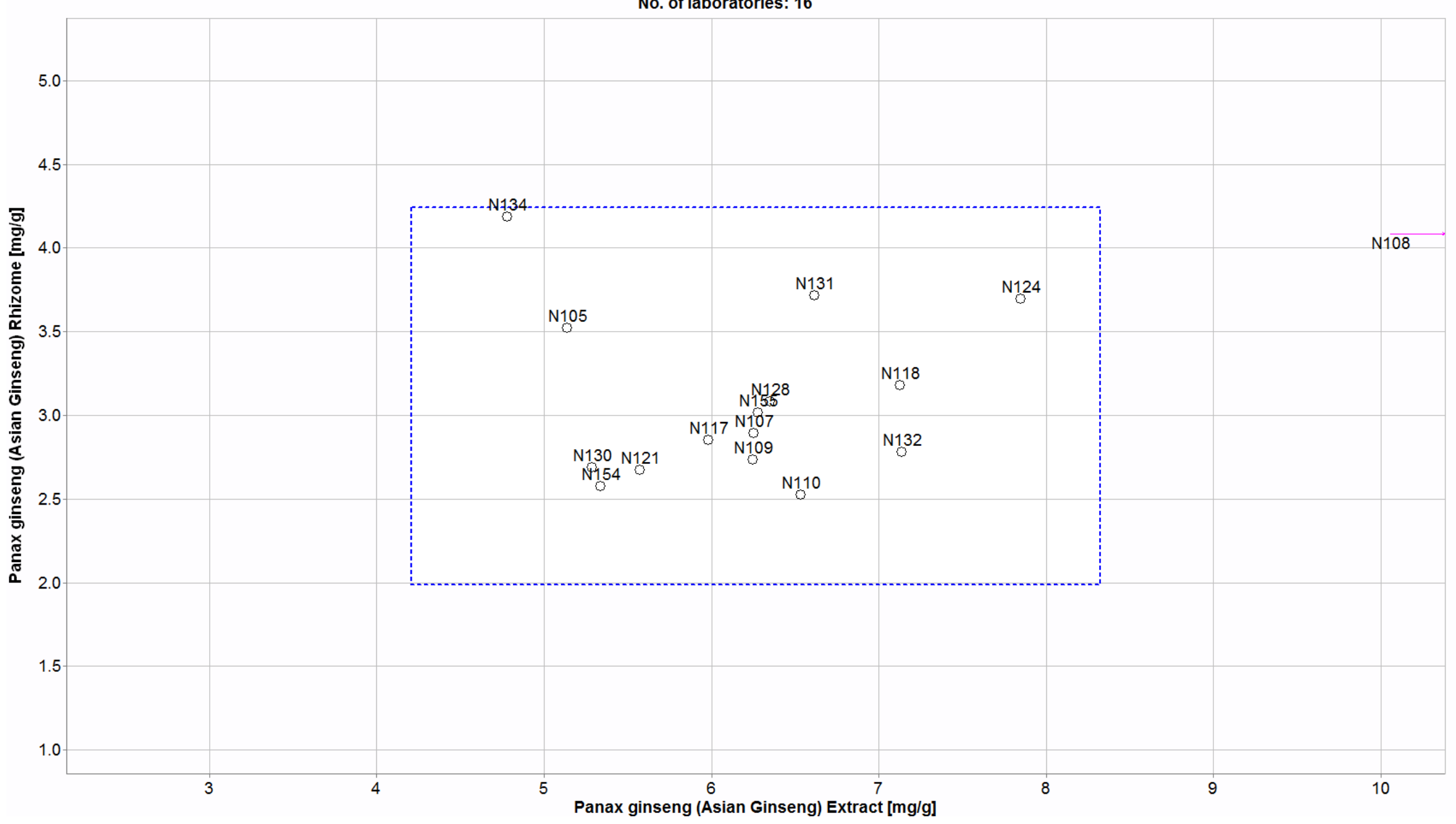

Figure 63. Laboratory means for ginsenoside $\mathrm{Rg} 1$ in Asian ginseng rhizome and Asian ginseng extract (sample/sample comparison view). In this view, the individual laboratory mean for one sample (rhizome) is compared to the mean for a second sample (extract). The dotted blue box represents the consensus range of tolerance for Asian ginseng extract (x-axis) and Asian ginseng rhizome ( $\mathrm{y}$-axis), calculated as the values above and below the consensus means that result in an acceptable $Z_{\text {comm }}^{\prime}$ score, $\left|Z_{\text {comm }}^{\prime}\right| \leq 2$. 


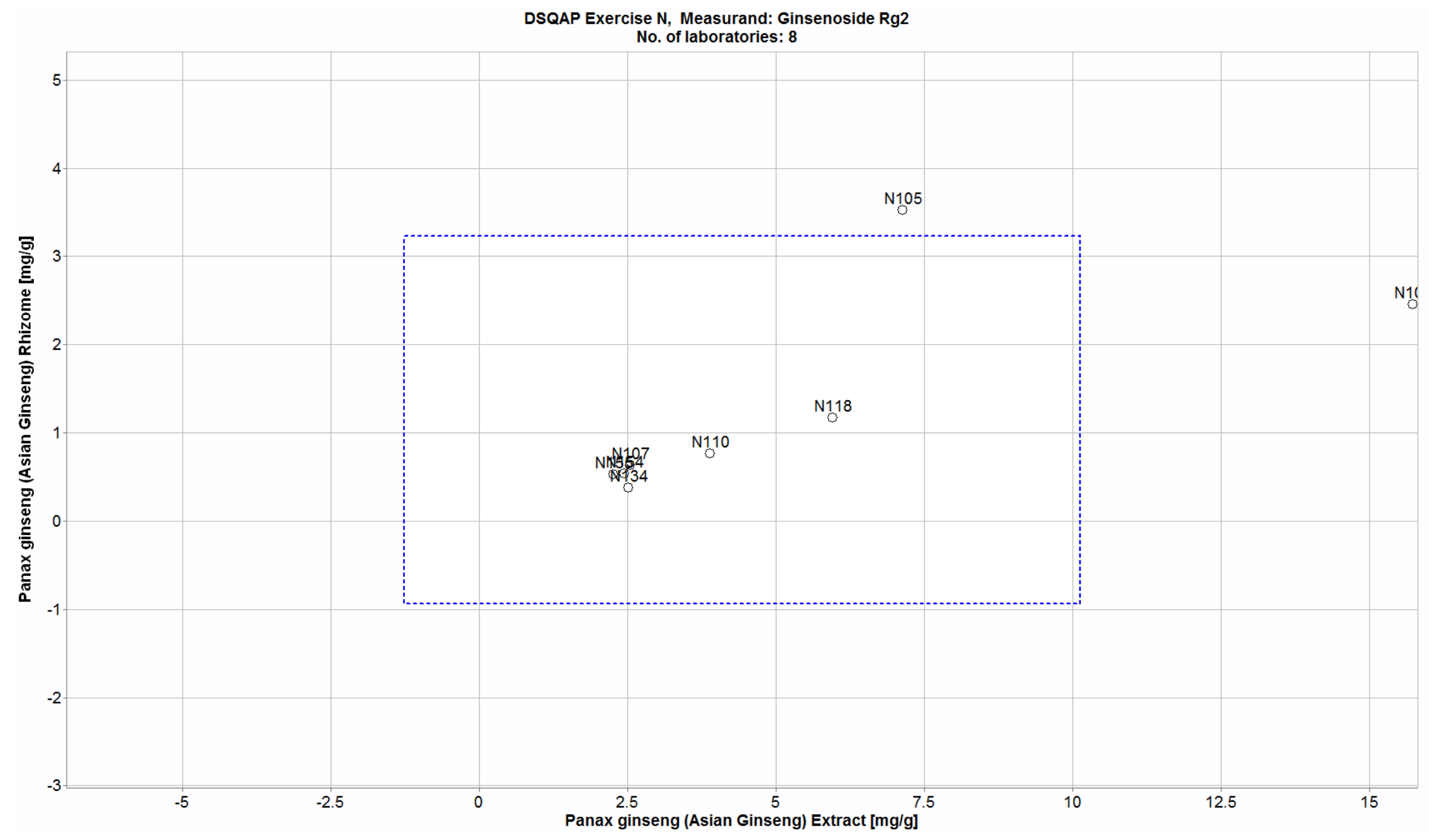

Figure 64. Laboratory means for ginsenoside Rg2 in Asian ginseng rhizome and Asian ginseng extract (sample/sample comparison view). In this view, the individual laboratory mean for one sample (rhizome) is compared to the mean for a second sample (extract). The dotted blue box represents the consensus range of tolerance for Asian ginseng extract (x-axis) and Asian ginseng rhizome (y-axis), calculated as the values above and below the consensus means that result in an acceptable $Z_{\text {comm }}^{\prime}$ score, $\left|Z_{\text {comm }}^{\prime}\right| \leq 2$. 